JOURNAL OF THE AMERICAN MATHEMATICAL SOCIETY

Volume 19, Number 4, October 2006, Pages 815-920

S 0894-0347(06)00524-8

Article electronically published on February 20, 2006

\title{
STABLE MANIFOLDS FOR ALL MONIC SUPERCRITICAL FOCUSING NONLINEAR SCHRÖDINGER EQUATIONS IN ONE DIMENSION
}

\author{
J. KRIEGER AND W. SCHLAG
}

\section{INTRODUCTION}

We consider the nonlinear Schrödinger equation

$$
i \partial_{t} \psi+\partial_{x}^{2} \psi=-|\psi|^{2 \sigma} \psi
$$

on the line $\mathbb{R}$ with $\sigma>2$. This is exactly the $L^{2}$-supercritical case and these equations are locally well-posed in $H^{1}(\mathbb{R})=W^{1,2}(\mathbb{R})$. Let $\phi=\phi(\cdot, \alpha)$ be the ground state of

$$
-\phi^{\prime \prime}+\alpha^{2} \phi=\phi^{2 \sigma+1} \text {. }
$$

By this we mean that $\phi>0$ and $\phi \in C^{2}(\mathbb{R})$. It is a classical fact that such solutions exist and are unique up to translations. In fact, for the case $\alpha=1$ the solutions are

$$
\phi(x, 1)=\frac{(\sigma+1)^{\frac{1}{2 \sigma}}}{\cosh ^{\frac{1}{\sigma}}(\sigma x)},
$$

whereas for general $\alpha>0$ they are obtained from this solution by rescaling: $\phi(x, \alpha)=\alpha^{\frac{1}{\sigma}} \phi(\alpha x, 1)$.

Clearly, the standing wave $\psi=e^{i t \alpha^{2}} \phi$ solves (1.1). We seek an $H^{1}$-solution $\psi$ of the form $\psi=W+R$ where

$$
\begin{aligned}
W(t, x) & =e^{i \theta(t, x)} \phi(x-y(t), \alpha(t)), \\
\theta(t, x) & =v(t) x-\int_{0}^{t}\left(v(s)^{2}-\alpha^{2}(s)\right) d s+\gamma(t), \\
y(t) & =2 \int_{0}^{t} v(s) d s+D(t)
\end{aligned}
$$

is the usual standing wave with a moving set of parameters $\pi(t):=(\gamma, v, D, \alpha)(t)$, and $R$ is a small perturbation. Performing a Galilei transform, we may assume that $W(0, x)=\phi(x, \alpha)$.

Received by the editors January 13, 2005.

2000 Mathematics Subject Classification. Primary 35Q55, 35Q51, 37K40, 37K45.

Key words and phrases. Critical Schrödinger equation, stable manifolds, modulation theory, spectral theory.

The first author was partially supported by the NSF grant DMS-0401177. He also wishes to thank Caltech, where part of this work was done.

The second author was partially supported by the NSF grant DMS-0300081 and a Sloan fellowship.

(C)2006 American Mathematical Society Reverts to public domain 28 years from publication 
Theorem 1.1. Fix any $\sigma>2$ in (1.1) and any $\alpha_{0}>0$. Let

$$
\Sigma:=\left\{f \in L^{2}(\mathbb{R}) \mid\|f\|<\infty\right\}
$$

where1

and set

$$
\|f\|:=\|f\|_{H^{1}}+\|\langle x\rangle f\|_{L^{1} \cap L^{2}}+\left\|\langle x\rangle \partial_{x} f\right\|_{L^{1}}
$$

Then there exist a real-linear subspace $\mathcal{S} \subset \Sigma$ of codimension five and a small $\delta>0$ with the following properties: there exists a map $\Phi: \mathcal{B} \cap \mathcal{S} \rightarrow \Sigma$ such that 2

$$
\begin{aligned}
\left\|\Phi\left(R_{0}\right)\right\| & \lesssim\left\|R_{0}\right\|^{2} \quad \forall R_{0} \in \mathcal{B} \cap \mathcal{S}, \\
\left\|\Phi\left(R_{0}\right)-\Phi\left(\tilde{R}_{0}\right)\right\| & \lesssim \delta\left\|R_{0}-\tilde{R}_{0}\right\| \quad \forall R_{0}, \tilde{R}_{0} \in \mathcal{B} \cap \mathcal{S}
\end{aligned}
$$

and so that for any $R_{0} \in \mathcal{B} \cap \mathcal{S}$ the PDE (1.1) has a global $H^{1}$ solution $\psi(t)$ for $t \geq 0$ with initial condition $\psi(0)=\phi\left(\cdot, \alpha_{0}\right)+R_{0}+\Phi\left(R_{0}\right)$. Moreover,

$$
\psi(t)=W(t, \cdot)+R(t)
$$

where $W$ as in (1.3) is governed by a path $\pi(t)$ of parameters, $\pi(0)=\left(0,0,0, \alpha_{0}\right)$, and which converges to some terminal vector $\pi(\infty)$ with $\sup _{t \geq 0}|\pi(t)-\pi(\infty)| \lesssim \delta^{2}$ and so that

$$
\|R(t)\|_{H^{1}} \lesssim \delta, \quad\|R(t)\|_{\infty} \lesssim \delta\langle t\rangle^{-\frac{1}{2}}, \quad\left\|\langle x-y(t)\rangle^{-\frac{1}{2}-\varepsilon} R(t)\right\|_{\infty} \lesssim \delta\langle t\rangle^{-1-\varepsilon}
$$

for all $t>0$ and some $\varepsilon>0$. The solution $\psi(t)$ is unique amongst all solutions with these initial data and satisfying the above decay assumptions as well as certain orthogonality relations and decay assumptions on the path (which will be specified later). Finally, there is scattering:

$$
R(t)=e^{i t \partial_{x}^{2}} f_{0}+o_{L^{2}}(1) \text { as } t \rightarrow \infty
$$

for some $f_{0} \in L^{2}(\mathbb{R})$.

It is well known that the supercritical equation (1.1) is orbitally unstable; see Berestycki and Cazenave [3. This is in contrast to the orbital stability of the subcritical equations that was proved by Cazenave and Lions [7] and Weinstein [55], [56]. In fact, $\left[3\right.$ shows that one can have finite time blow-up for initial data $\psi_{0}=\phi(\cdot, \alpha)+R_{0}$ where $R_{0}$ can be made arbitrarily small in any reasonable norm. Theorem 1.1 states that we do have the same asymptotic stability and scattering as in the subcritical case, provided we choose our initial data on a suitable submanifold. In addition to the aforementioned references, there is of course a vast literature on the (in)stability of standing waves for nonlinear evolution equations. The orbital stability question (for Klein-Gordon, NLS, as well as many other classes of PDE) was addressed by Shatah [46], Shatah and Strauss [47], Weinstein [55], [56], Grillakis, Shatah, and Strauss [22], 23] (who developed an "abstract" theory of orbital stability), Grillakis [21], and Comech and Pelinovsky [9]. As far as the question of asymptotic stability is concerned (which is much closer to the present paper), see Soffer and Weinstein [49, [50, Buslaev and Perelman [5], 6], Cuccagna [10, Rodnianski, Schlag, and Soffer [42, 43, Perelman [35, 36], 37, Fröhlich, Jonsson, Gustafson,

\footnotetext{
${ }^{1}$ The weight $\langle x\rangle$ in the definition of $\|\cdot\|$ can be replaced with a more slowly growing weight, but we keep it in this form for aesthetic purposes.

${ }^{2}$ Whenever we use the symbol $\lesssim$, certain universal multiplicative constants are implied which do not depend on any varying parameters appearing in the proof.
} 
Sigal [16, Fröhlich, Tsai, and Yau [17, as well as Gang and Sigal 18, 19]. For surveys of some of this material, see Strauss [51] and Sulem and Sulem [52].

To understand the origin of the linear subspace $\mathcal{S}$ in our theorem, we associate with each $\alpha>0$ the matrix operator

$$
\mathcal{H}=\mathcal{H}(\alpha)=\left(\begin{array}{cc}
-\partial_{x}^{2}+\alpha^{2}-(\sigma+1) \phi^{2 \sigma}(\cdot, \alpha) & -\sigma \phi^{2 \sigma}(\cdot, \alpha) \\
\sigma \phi^{2 \sigma}(\cdot, \alpha) & \partial_{x}^{2}-\alpha^{2}+(\sigma+1) \phi^{2 \sigma}(\cdot, \alpha)
\end{array}\right) .
$$

This operator arises by linearizing the NLS equation (1.1) around a standing wave. It is closed on the domain $W^{2,2}(\mathbb{R}) \times W^{2,2}(\mathbb{R})$ and its spectrum has the following form: It is located on $\mathbb{R} \cup i \mathbb{R}$, with essential spectrum equal to $\left(-\infty,-\alpha^{2}\right] \cup\left[\alpha^{2}, \infty\right)$. The discrete spectrum equals $\{0, \pm i \gamma(\alpha)\}$, where $\gamma(\alpha)=\alpha^{2} \gamma(1)>0$. Both $\pm i \gamma(\alpha)$ are simple eigenvalues with exponentially decaying eigenfunctions, whereas 0 is an eigenvalue of geometric multiplicity two and algebraic multiplicity four (the latter fact goes back to Weinstein [55]).

Next, we introduce the Riesz projection $P_{u}^{+}(\alpha)$ such that

$$
\operatorname{spec}\left(\mathcal{H}(\alpha) P_{u}^{+}(\alpha)\right)=\{0\} \cup\{i \gamma(\alpha)\} .
$$

The notation $P_{u}^{+}$is meant to indicate the unstable modes as $t \rightarrow+\infty$. The reallinear, finite-codimensional subspace $\mathcal{S}$ above is precisely the set of $R_{0} \in \Sigma$ so that 3

$$
P_{u}^{+}\left(\alpha_{0}\right)\left(\begin{array}{c}
R_{0} \\
\bar{R}_{0}
\end{array}\right)=0
$$

The codimension of $\mathcal{S}$ is the number of unstable (or nondecaying) modes of the linearization as $t \rightarrow \infty$ : four in the root space and one exponentially unstable mode. The stable manifold $\mathcal{M}$ is the surface described by the parameterization $R_{0} \mapsto R_{0}+\Phi\left(R_{0}\right)$ where $R_{0}$ belongs to a small ball $\mathcal{B} \cap \mathcal{S}$ inside of $\mathcal{S}$. The inequality (1.7) means that $\mathcal{S}$ is the tangent space to $\mathcal{M}$ at zero, whereas (1.8) expresses that $\mathcal{M}$ is given in terms of a Lipschitz parameterization. It is easy to see that $\mathcal{M}$ is the graph of a Lipschitz map $\tilde{\Phi}: \mathcal{S} \cap \mathcal{B} \rightarrow \Sigma$. Indeed, define $\tilde{\Phi}$ as

$$
R_{0}+P_{\mathcal{S}} \Phi\left(R_{0}\right) \mapsto R_{0}+\Phi\left(R_{0}\right),
$$

where $P_{\mathcal{S}}$ is the projection onto $\mathcal{S}$ which is induced by the Riesz-projection $I-$ $P_{u}^{+}\left(\alpha_{0}\right)$ (the latter operates on $L^{2} \times L^{2}$, whereas we need only the first coordinate of this projection; see Remark 9.5 below for the details of this). The left-hand side is clearly in $\mathcal{S}$. Moreover, to see that this map is well defined as well as Lipschitz, note that (1.8) implies that

$$
\begin{aligned}
& (1-C \delta)\left\|R_{1}-R_{0}\right\| \leq\left\|R_{1}-R_{0}+\Phi\left(R_{1}\right)-\Phi\left(R_{0}\right)\right\| \leq(1+C \delta)\left\|R_{1}-R_{0}\right\|, \\
& (1-C \delta)\left\|R_{1}-R_{0}\right\| \leq\left\|R_{1}-R_{0}+P_{\mathcal{S}} \Phi\left(R_{1}\right)-P_{\mathcal{S}} \Phi\left(R_{0}\right)\right\| \leq(1+C \delta)\left\|R_{1}-R_{0}\right\| .
\end{aligned}
$$

Since the root-space of $\mathcal{H}(\alpha)$ at zero does not destroy asymptotic stability or instability in the subcritical case, one would expect that the new unstable phenomena in the supercritical case should only be connected with the imaginary eigenvalues of $\mathcal{H}(\alpha)$. More precisely, since we are considering $t \rightarrow+\infty$, they should result exclusively from the eigenvalue $i \gamma$, where $\gamma>0$. Hence, the true codimension of our stable manifold should be one. In the following theorem we obtain such a stable hypersurface, by applying the three-parameter family of Galilei transforms

\footnotetext{
${ }^{3}$ We will show below that $P_{u}^{+}: \tilde{\Sigma} \rightarrow \tilde{\Sigma}$ where $\tilde{\Sigma}=\left\{\left(\frac{f}{f}\right): f \in \Sigma\right\}$.
} 
together with scaling to the manifold $\mathcal{M}$ from Theorem 1.1. Since this family acts transversally to $\mathcal{M}$, we recover four of the missing dimensions this way.

Theorem 1.2. Fix any $\alpha_{0}>0$. Then there exist a small $\delta>0$ and a Lipschitz manifold $\mathcal{N}$ inside the space $\Sigma$ of sizd $\delta$ and codimension one so that $\phi\left(\cdot, \alpha_{0}\right) \in \mathcal{N}$ with the following property: for any choice of initial data $\psi(0) \in \mathcal{N}$ the PDE (1.1) has a global $H^{1}$ solution $\psi(t)$ for $t \geq 0$. Moreover,

$$
\psi(t)=W(t, \cdot)+R(t)
$$

where $W$ as in (1.3) is governed by a path $\pi(t)$ of parameters so that $\mid \pi(0)-$ $\left(0,0,0, \alpha_{0}\right) \mid \lesssim \delta$ and which converges to some terminal vector $\pi(\infty)$ such that $\sup _{t \geq 0}|\pi(t)-\pi(\infty)| \lesssim \delta^{2}$. The solution is unique under the same conditions as in the preceding theorem. Finally, (1.9) holds and there is scattering:

$$
R(t)=e^{i t \partial_{x}^{2}} f_{0}+o_{L^{2}}(1) \quad \text { as } t \rightarrow \infty
$$

for some $f_{0} \in L^{2}(\mathbb{R})$.

This result raises the interesting question of deciding the behavior of solutions with initial data $\phi(0) \in \mathcal{B} \backslash \mathcal{N}$. It is known that at least for some choices of such initial data the solution blows up, but the authors do not know what to expect in general.

Bates and Jones 2 studied the questions of stable manifolds for evolution equations in great generality. They proved the existence of stable, unstable, and center stable manifolds (which are also Lipschitz) for abstract evolution equations under certain conditions on the linear part of the evolution. Later Gesztesy, Jones, Latushkin, and Stanislavova 20] verified these conditions for a class of nonlinear Schrödinger equations which are obtained by linearizing around standing waves. Thus, they obtain the existence of such a dynamical splitting of the space of initial data close to a standing wave. The notion of stability used by these authors is the orbital one. Hence, in contrast to our theorems, no asymptotic convergence or scattering statements are made. Rather, their results are of a perturbative and spectral nature situated in energy space. For yet more related results, see the paper by Pillet and Wayne [38, as well as the book by Li and Wiggins [30]. Tsai and Yau [53] investigated the question of stable manifolds for NLS equations with a potential which admit excited states. These are standing waves which are generated by bifurcations off bound states of the linear Schrödinger operator. Such excited states are unstable as they will tend to collapse to the ground state. Tsai and Yau obtain conditional stability of these excited states.

Theorems 1.1 and 1.2 (but with unweighted norms) were first proved in three dimensions in [44] for the cubic focusing NLS equation, albeit under the assumption that there are no imbedded eigenvalues in the essential spectrum of the linearized operators. The present paper is related to [44, although it does differ in several important aspects. First, all spectral properties are proved here analytically, whereas 44] required verifying some spectral properties of the well-known pair of Schrödinger operators $L_{-}$and $L_{+}$numerically; see [13]. Moreover, the absence of

\footnotetext{
${ }^{4}$ This means that $\mathcal{N}$ is the graph of a Lipschitz map $\Psi$ with domain $\mathcal{B} \cap \tilde{\mathcal{S}}$ where $\tilde{\mathcal{S}}$ is a subspace of codimension one and with $\mathcal{B}$ as in (1.6).
} 
imbedded eigenvalues for the systems remained an assumption (although it should be a generic assumption since imbedded eigenvalues are unstable; see Cuccagna, Pelinovsky, and Vougalter [12, 11]). Second, the (free) dispersive decay in one dimension is $t^{-\frac{1}{2}}$, which is not integrable at infinity. This forces us to use an improved decay estimate which takes the form

$$
\left\|\langle x\rangle^{-1} e^{i t\left(-\partial_{x}^{2}+V\right)} P_{c} f\right\|_{L^{\infty}(\mathbb{R})} \leq C t^{-\frac{3}{2}}\|\langle x\rangle f\|_{L^{1}(\mathbb{R})}
$$

for scalar operators $-\partial_{x}^{2}+V$ with no resonance at zero; see [45]. Weaker forms of this result on $L^{2}(\mathbb{R})$ and with higher weights were discovered by Murata 34 . and later reproved by Buslaev and Perelman [5] for Schrödinger systems. However, due to the fact that we cover here the full supercritical range, we need to rely on this estimate (or interpolates of it) rather than any weaker version, albeit for nonselfadjoint matrix operators rather than scalar operators. The transition from the scalar case (as in 45, say) to the matrix case requires a more sophisticated functional framework, which involves developing the scattering theory (i.e., Jost solutions) of these matrix Schrödinger operators as in [5]. Since the estimates we require here are considerably sharper than the ones in Buslaev-Perelman, we carefully develop this framework, together with the necessary spectral theory in the second half of the paper. This part is of independent interest. As far as the nonlinear argument is concerned, the weights inside of our norms will force us to depart from the contraction procedure employed in [44, which takes place in a fixed Banach space, and use a method of iteration which adjusts the Banach space depending on the iterate. This issue here is that the weights need to be centered around a path $y_{j}(t)$. It is impossible to compare these norms for large $t$ because these paths will then be separated by a distance exceeding one. This forces us to truncate at a time $T_{j}$ which grows with $j$. This method, which is more involved than the contraction from [44, is of independent interest.

Finally, we would like to point out that the $L^{2}$-critical case is studied in the companion paper [29]. There one is concerned with the question of conditional asymptotic stability of the pseudo-conformal blow-up solutions (see Bourgain and Wang 4] for earlier work in that direction). The latter are known to be unstable; see Perelman [36, Merle and Raphael [31, [32, 33], and Raphael [39. The critical equation is much more involved than the supercritical one, both conceptually and technically. For example, one looses hyperbolicity; cf. Lemma 4.6 below. In addition, in the critical case many estimates require exploiting cancelation effects (a "nullstructure" needs to be found for that purpose).

\section{The linearization, Galilei transforms, and $\mathcal{J}$-invariance}

We begin by linearizing around the standing wave.

Lemma 2.1. Let $0<T \leq \infty$. Assume that $\pi(t)=(\gamma(t), v(t), D(t), \alpha(t)):[0, T) \rightarrow$ $\mathbb{R}^{4}$ belongs to $C^{1}\left([0, T), \mathbb{R}^{4}\right)$, and let $W=W(t, x)$ be as in (1.3). Then $\psi \in$ $C\left([0, T), H^{1}(\mathbb{R})\right) \cap C^{1}\left([0, T), H^{-1}(\mathbb{R})\right)$ solves (1.1) with $\psi=W+R$ iff $Z=\left(\begin{array}{l}R \\ \bar{R}\end{array}\right)$ solves the equation

$$
i \partial_{t} Z+\left(\begin{array}{cc}
\partial_{x}^{2}+(\sigma+1)|W|^{2 \sigma} & \sigma|W|^{2(\sigma-1)} W^{2} \\
-\sigma|W|^{2(\sigma-1)} W^{2} & -\partial_{x}^{2}-(\sigma+1)|W|^{2 \sigma}
\end{array}\right) Z=-i \dot{\pi} \partial_{\pi} W+N(Z, W)
$$


in the sense of $C\left([0, T), H^{1}(\mathbb{R}) \times H^{1}(\mathbb{R})\right) \cap C^{1}\left([0, T), H^{-1}(\mathbb{R}) \times H^{-1}(\mathbb{R})\right)$ where (2.1)

$$
\begin{aligned}
- & \dot{v}\left(\begin{array}{c}
-x e^{i \theta} \phi(\cdot-y, \alpha) \\
x e^{-i \theta} \phi(\cdot-y, \alpha)
\end{array}\right)-\dot{\gamma}\left(\begin{array}{c}
-e^{i \theta} \phi(\cdot-y, \alpha) \\
e^{-i \theta} \phi(\cdot-y, \alpha)
\end{array}\right) \\
& -i \dot{\alpha}\left(\begin{array}{c}
e^{i \theta} \partial_{\alpha} \phi(\cdot-y, \alpha) \\
e^{-i \theta} \partial_{\alpha} \phi(\cdot-y, \alpha)
\end{array}\right)-i \dot{D}\left(\begin{array}{c}
-e^{i \theta} \partial_{x} \phi(\cdot-y, \alpha) \\
-e^{-i \theta} \partial_{x} \phi(\cdot-y, \alpha)
\end{array}\right) \\
& +\left(\begin{array}{c}
-|R+W|^{2 \sigma}(R+W)+|W|^{2 \sigma} W+(\sigma+1)|W|^{2 \sigma} R+\sigma|W|^{2(\sigma-1)} W^{2} \bar{R} \\
|R+W|^{2 \sigma}(\bar{R}+\bar{W})-|W|^{2 \sigma} \bar{W}-(\sigma+1)|W|^{2 \sigma} \bar{R}-\sigma|W|^{2(\sigma-1)} \bar{W}^{2} R
\end{array}\right) \\
= & :-i \dot{\pi} \partial_{\pi} W+N(Z, W) .
\end{aligned}
$$

Here $y$ and $\theta$ are the functions from (1.5) and (1.4), and $\alpha=\alpha(t)$. For future reference, we denote the matrix operator on the left-hand side of (2.1) by $-\mathcal{H}(\pi(t))$, i.e.,

$$
\mathcal{H}(\pi(t)):=\left(\begin{array}{cc}
-\partial_{x}^{2}-(\sigma+1)|W|^{2 \sigma} & -\sigma|W|^{2(\sigma-1)} W^{2} \\
\sigma|W|^{2(\sigma-1)} \bar{W}^{2} & \partial_{x}^{2}+(\sigma+1)|W|^{2 \sigma}
\end{array}\right)
$$

The nonlinear term in (2.1), which we denote by $N(Z, W)=\left(\begin{array}{l}N_{1}(Z, W) \\ N_{2}(Z, W)\end{array}\right)$, is quadratic in $R, \bar{R}$. This means that

$$
N(0, W)=\partial_{R} N(0, W)=\partial_{\bar{R}} N(0, W)=0 .
$$

Proof. It will be convenient to consider the more general NLS equation

$$
i \partial_{t} \psi+\partial_{x}^{2} \psi=-\beta\left(|\psi|^{2}\right) \psi
$$

Let $\phi=\phi(\cdot, \alpha(t))$ for ease of notation. Direct differentiation shows that $W(t, x)$ satisfies

$$
i \partial_{t} W+\partial_{x}^{2} W=-\beta\left(|W|^{2}\right) W-W(\dot{v} x+\dot{\gamma})-i e^{i \theta} \partial_{x} \phi \cdot \dot{D}+i e^{i \theta} \dot{\alpha} \partial_{\alpha} \phi .
$$

Hence $W+R$ is a solution of (2.3) iff

$i \partial_{t} R+\partial_{x}^{2} R=-\beta\left(|W+R|^{2}\right)(W+R)+\beta\left(|W|^{2}\right) W+e^{i \theta} \phi(\dot{v} x+\dot{\gamma})+i e^{i \theta} \partial_{x} \phi \cdot \dot{D}-i e^{i \theta} \dot{\alpha} \partial_{\alpha} \phi$.

Joining this equation with its conjugate leads to the system

$$
\begin{aligned}
i \partial_{t} Z+\left(\begin{array}{cc}
\partial_{x}^{2}+\beta^{\prime}\left(|W|^{2}\right)|W|^{2}+\beta\left(|W|^{2}\right) & \beta^{\prime}\left(|W|^{2}\right) W^{2} \\
-\beta^{\prime}\left(|W|^{2}\right) W^{2} & -\partial_{x}^{2}-\beta^{\prime}\left(|W|^{2}\right)|W|^{2}-\beta\left(|W|^{2}\right)
\end{array}\right) Z \\
=-\dot{v}\left(\begin{array}{c}
-x e^{i \theta} \phi(\cdot-y, \alpha) \\
x e^{-i \theta} \phi(\cdot-y, \alpha)
\end{array}\right)-\dot{\gamma}\left(\begin{array}{c}
-e^{i \theta} \phi(\cdot-y, \alpha) \\
e^{-i \theta} \phi(\cdot-y, \alpha)
\end{array}\right) \\
\quad-i \dot{\alpha}\left(\begin{array}{c}
e^{i \theta} \partial_{\alpha} \phi(\cdot-y, \alpha) \\
e^{-i \theta} \partial_{\alpha} \phi(\cdot-y, \alpha)
\end{array}\right)-i \dot{D}\left(\begin{array}{c}
-e^{i \theta} \partial_{x} \phi(\cdot-y, \alpha) \\
-e^{-i \theta} \partial_{x} \phi(\cdot-y, \alpha)
\end{array}\right) \\
\quad+\left(\begin{array}{c}
-\beta\left(|R+W|^{2}\right)(R+W)+\beta\left(|W|^{2}\right) W+\left[\beta^{\prime}\left(|W|^{2}\right)|W|^{2}+\beta\left(|W|^{2}\right)\right] R+\beta^{\prime}\left(|W|^{2}\right) W^{2} \bar{R} \\
\beta\left(|R+W|^{2}\right)(\bar{R}+\bar{W})-\beta\left(|W|^{2}\right) \bar{W}-\left[\beta^{\prime}\left(|W|^{2}\right)|W|^{2}+\beta\left(|W|^{2}\right)\right] \bar{R}-\beta^{\prime}\left(|W|^{2}\right) \bar{W}^{2} R
\end{array}\right) .
\end{aligned}
$$

Conversely, if $Z(0)$ is of the form

$$
Z(0)=\left(\frac{Z_{1}(0)}{Z_{1}(0)}\right)
$$

and $Z(t)$ solves (2.4), then $Z(t)$ remains of this form for all times. This is simply the statement that the system (2.4) is invariant under the transformation

$$
\mathcal{J}: f \mapsto \overline{J f}, \quad J=\left(\begin{array}{cc}
0 & 1 \\
1 & 0
\end{array}\right), \quad f=\left(\frac{f_{1}}{f_{1}}\right),
$$


which can be checked by direct verification. This fact allows us to go back from the system to the scalar equation (2.3). Finally, it is easy to see that the nonlinear term in (2.4) is quadratic and that (2.4) reduces to (2.1) if $\beta(u)=u^{\sigma}$ for all $u>0$.

Remark 2.2. Notice that we have the equality

$$
\begin{aligned}
& \dot{v}\left(\begin{array}{c}
-x e^{i \theta} \phi(\cdot-y, \alpha) \\
x e^{-i \theta} \phi(\cdot-y, \alpha)
\end{array}\right)+\dot{\gamma}\left(\begin{array}{c}
-e^{i \theta} \phi(\cdot-y, \alpha) \\
e^{-i \theta} \phi(\cdot-y, \alpha)
\end{array}\right)+i \dot{\alpha}\left(\begin{array}{c}
e^{i \theta} \partial_{\alpha} \phi(\cdot-y, \alpha) \\
e^{-i \theta} \partial_{\alpha} \phi(\cdot-y, \alpha)
\end{array}\right) \\
& +i \dot{D}\left(\begin{array}{c}
-e^{i \theta} \partial_{x} \phi(\cdot-y, \alpha) \\
-e^{-i \theta} \partial_{x} \phi(\cdot-y, \alpha)
\end{array}\right) \\
& =\dot{v}\left(\begin{array}{c}
-(x-y) e^{i \theta} \phi(\cdot-y, \alpha) \\
(x-y) e^{-i \theta} \phi(\cdot-y, \alpha)
\end{array}\right)+[\dot{\gamma}+\dot{v} y]\left(\begin{array}{c}
-e^{i \theta} \phi(\cdot-y, \alpha) \\
e^{-i \theta} \phi(\cdot-y, \alpha)
\end{array}\right) \\
& +i \dot{\alpha}\left(\begin{array}{c}
e^{i \theta} \partial_{\alpha} \phi(\cdot-y, \alpha) \\
e^{-i \theta} \partial_{\alpha} \phi(\cdot-y, \alpha)
\end{array}\right)+i \dot{D}\left(\begin{array}{c}
-e^{i \theta} \partial_{x} \phi(\cdot-y, \alpha) \\
-e^{-i \theta} \partial_{x} \phi(\cdot-y, \alpha)
\end{array}\right)
\end{aligned}
$$

Abusing notation, we shall later refer to this expression as $i \dot{\tilde{\pi}} \partial_{\pi} W$, where

$$
\dot{\tilde{\pi}}:=(\dot{\gamma}+\dot{v} y, \dot{v}, \dot{D}, \dot{\alpha}) \text {. }
$$

The quantity $\dot{\tilde{\pi}}$ shall play an important role in the argument to follow.

The $\mathcal{J}$-invariant vectors in $L^{2}(\mathbb{R}) \times L^{2}(\mathbb{R})$ form a real-linear subspace, namely

$$
\left\{\left(\begin{array}{l}
f \\
\bar{f}
\end{array}\right) \mid f \in L^{2}(\mathbb{R})\right\} \text {. }
$$

If we write $f=f_{1}+i f_{2}$, it can be seen to be isomorphic to the subspace

$$
\left\{\left(\begin{array}{l}
f_{1} \\
f_{2}
\end{array}\right) \mid f_{1}, f_{2} \in L^{2}(\mathbb{R}), f_{1}, f_{2} \text { are real-valued }\right\}
$$

which is clearly linear, but only over $\mathbb{R}$. Throughout the paper, we need to insure that all vectorial solutions we construct belong to this subspace. Only then is it possible to revert back to the scalar NLS (1.1).

To perform estimates, one needs to transform (2.1) to a resting frame. This requires certain properties of the path $\pi(t)$. In the following definition, $\varepsilon>0$ and $\delta>0$ are fixed small constants.

Definition 2.3. Let $T>0$, to be thought of as a large number 5 . We say that a path $\pi:[0, T] \rightarrow \mathbb{R}^{4}$ with $\pi(t):=(\gamma(t), v(t), D(t), \alpha(t))$ is admissible provided it belongs to $C^{1}\left([0, T], \mathbb{R}^{4}\right)$ and the estimate $|\dot{\pi}(t)| \leq \delta^{2}\langle t\rangle^{-2-\varepsilon}$ holds. Define a constant parameter vector $\pi_{T}=\left(\gamma_{T}, v_{T}, D_{T}, \alpha_{T}\right)$ as

$$
\begin{aligned}
\gamma_{T} & :=\gamma(T)+2 \int_{0}^{T} \int_{t}^{T}(v(s) \dot{v}(s)-\alpha(s) \dot{\alpha}(s)) d s d t, \\
v_{T} & :=v(T), \\
D_{T} & :=D(T)-2 \int_{0}^{T} \int_{t}^{T} \dot{v}(s) d s d t \\
\alpha_{T} & :=\alpha(T) .
\end{aligned}
$$

\footnotetext{
${ }^{5}$ The logic here is that we are working on a large time interval $[0, T]$ and are not concerned about what happens for times larger than $T$. In the iteration, we will adjust this parameter to the stage of the iteration we are at. Of course, $T \rightarrow \infty$ in this process.
} 
The logic behind these asymptotic parameters can be seen in the following lemma.

Lemma 2.4. Suppose $\pi$ is an admissible path and let $\theta, y$ and $\theta_{T}, y_{T}$ be as in (1.4), (1.5). Furthermore, define

$$
y_{T}(t):=2 t v_{T}+D_{T}, \theta_{T}(t, x):=v_{T} x-t\left(v_{T}^{2}-\alpha_{T}^{2}\right)+\gamma_{T}
$$

and

$$
\rho_{T}(t, x):=\theta\left(t, x+y_{T}\right)-\theta_{T}\left(t, x+y_{T}\right)
$$

Then

$$
\left|\rho_{T}(t, x)\right| \leq C_{\varepsilon} \delta^{2}(1+|x|)\langle t\rangle^{-\varepsilon}, \quad\left|y(t)-y_{T}(t)\right| \leq C_{\varepsilon} \delta^{2}\langle t\rangle^{-\varepsilon},
$$

as well as

$$
\left|\dot{\rho}_{T}(t, x)\right| \leq C_{\varepsilon} \delta^{2}(1+|x|)\langle t\rangle^{-1-\varepsilon}, \quad\left|\dot{y}(t)-\dot{y}_{T}(t)\right| \leq C_{\varepsilon} \delta^{2}\langle t\rangle^{-1-\varepsilon}
$$

for all $t$ with $T \geq t \geq 0$. The constants here only depend on $\varepsilon$.

Proof. First,

$\theta_{T}\left(t, x+y_{T}\right)=v_{T}\left(x+2 t v_{T}+D_{T}\right)+t\left(-v_{T}^{2}+\alpha_{T}^{2}\right)+\gamma_{T}=t\left(-v_{T}^{2}+\alpha_{T}^{2}\right)+v_{T}\left(x+D_{T}\right)+\gamma_{T}$.

In view of the definition of $\pi_{T}$,

$$
\begin{aligned}
\theta( & \left.t, x+y_{T}\right)-\theta_{T}\left(t, x+y_{T}\right) \\
= & v(t)\left(x+2 t v_{T}+D_{T}\right)-\int_{0}^{t}\left(v(s)^{2}-\alpha^{2}(s)\right) d s+\gamma(t) \\
& -v_{T}\left(x+2 t v_{T}+D_{T}\right)+t\left(v_{T}^{2}-\alpha_{T}^{2}\right)-\gamma_{T} \\
= & \left(v(t)-v_{T}\right)\left(x+2 t v_{T}+D_{T}\right)+2 \int_{0}^{T} \int_{s}^{T}(v \dot{v}-\alpha \dot{\alpha})\left(s^{\prime}\right) d s^{\prime} d s \\
& -\gamma_{T}+\gamma(t)-2 \int_{t}^{T} \int_{s}^{T}(v \dot{v}-\alpha \dot{\alpha})\left(s^{\prime}\right) d s^{\prime} d s \\
= & \left(v(t)-v_{T}\right)\left(x+2 t v_{T}+D_{T}\right)-2 \int_{t}^{T} \int_{s}^{T}(v \dot{v}-\alpha \dot{\alpha})\left(s^{\prime}\right) d s^{\prime} d s \\
& -\gamma(T)+\gamma(t) .
\end{aligned}
$$

Using Definition 2.3 implies the desired bound on $\rho_{T}$. As for $y(t)-y_{T}(t)$, the definition of $D_{T}$ implies that

$y_{T}(t)-y(t)=2 t v_{T}+D_{T}-2 \int_{0}^{t} v(s) d s-D(t)=D(T)-D(t)-2 \int_{t}^{T} \int_{s}^{T} \dot{v}\left(s^{\prime}\right) d s^{\prime} d s$,

which implies the stated estimate for $0 \leq t \leq T$.

Remark 2.5. One has similar definitions and properties for $\pi_{\infty}=\left(\gamma_{\infty}, v_{\infty}, D_{\infty}, \alpha_{\infty}\right)$, etc. One then replaces $[0, T]$ by $[0, \infty)$.

With $\pi_{T}=\left(\gamma_{T}, v_{T}, D_{T}, \alpha_{T}\right)$ a constant vector, define the usual Galilei transform to be

$$
\mathfrak{g}_{T}(t)=e^{i\left(\gamma_{T}+v_{T} x-t\left|v_{T}\right|^{2}\right)} e^{-i\left(2 t v_{T}+D_{T}\right) p},
$$


where $p:=-i \frac{d}{d x}$. The action of $\mathfrak{g}_{T}(t)$ on functions is

$$
\left(\mathfrak{g}_{T}(t) f\right)(x)=e^{i\left(\gamma_{T}+v_{T} x-t v_{T}^{2}\right)} f\left(x-2 t v_{T}-D_{T}\right) .
$$

It is unitary on $L^{2}$ and isometric on all $L^{p}$, and the commutation property

$$
e^{i t \partial_{x}^{2}} \mathfrak{g}_{T}(0)=\mathfrak{g}_{T}(t) e^{i t \partial_{x}^{2}}
$$

holds. The inverse of $\mathfrak{g}_{T}(t)$ is

$$
\mathfrak{g}_{T}(t)^{-1}=e^{i\left(2 t v_{T}+D_{T}\right) p} e^{-i\left(\gamma_{T}+v_{T} x-t v_{T}^{2}\right)}=e^{-i\left(\gamma_{T}+v_{T} D_{T}+v_{T} x+t v_{T}^{2}\right)} e^{i\left(2 t v_{T}+D_{T}\right) p} .
$$

Moreover, the Galilei transform (2.16) generates a four-parameter family of standing waves: Let $\phi\left(\cdot, \alpha_{T}\right)$ be the ground state of (1.2) with $\alpha=\alpha_{T}$. Then

$$
W_{T}(t, \cdot)=\mathfrak{g}_{T}(t)\left[e^{i t \alpha_{T}^{2}} \phi\left(\cdot, \alpha_{T}\right)\right]
$$

solves (1.1), where $W_{T}$ is a standing wave as introduced in (1.3) but with the constant parameter path $\pi_{T}$. This can also be written as

$$
W_{T}(t, x)=e^{i \theta_{T}(t, x)} \phi\left(x-y_{T}(t), \alpha_{T}\right),
$$

where $y_{T}, \theta_{T}$ are as in Lemma 2.4. As usual, we transform (2.1) to a stationary frame by means of Galilei transforms. In addition, a modulation will be performed. The details are as follows.

Lemma 2.6. Let $\pi(t)$ and $\pi_{T}$ be as in Definition 2.3. Given $Z=\left(\begin{array}{l}Z_{1} \\ Z_{2}\end{array}\right)$, introduce $U$, as well as $M_{T}(t), \mathcal{G}_{T}(t)$, as

$$
U(t)=\left(\begin{array}{cc}
e^{i \omega_{T}(t)} & 0 \\
0 & e^{-i \omega_{T}(t)}
\end{array}\right)\left(\frac{\mathfrak{g}_{T}(t)^{-1} Z_{1}(t)}{\mathfrak{g}_{T}(t)^{-1} \overline{Z_{2}(t)}}\right)=M_{T}(t) \mathcal{G}_{T}(t) Z(t),
$$

where $\omega_{T}(t)=-t \alpha_{T}^{2}$. Then $Z(t)$ solves (2.1) in the $H^{1}$ sense iff $U=\left(\begin{array}{l}U_{1} \\ U_{2}\end{array}\right)$ as in (2.19) satisfies the following PDE in the $H^{1}$ sense (with $\phi_{T}=\phi\left(\cdot, \alpha_{T}\right)$ ):

$$
\begin{aligned}
& i \dot{U}(t)+\left(\begin{array}{cc}
\partial_{x}^{2}+(\sigma+1) \phi_{T}^{2 \sigma}-\alpha_{T}^{2} & \sigma \phi_{T}^{2 \sigma} \\
-\sigma \phi_{T}^{2 \sigma} & -\partial_{x}^{2}-(\sigma+1) \phi_{T}^{2 \sigma}+\alpha_{T}^{2}
\end{array}\right) U \\
& =-i \dot{\pi} \partial_{\pi} \tilde{W}_{T}(\pi)+N_{T}(U, \pi)+V_{T} U
\end{aligned}
$$

where

$$
\begin{aligned}
& V_{T}=V_{T}(t, x) \\
& :=\left(\begin{array}{cc}
(\sigma+1)\left(\phi_{T}^{2 \sigma}(x)-\phi^{2 \sigma}\left(x+y_{T}-y\right)\right) & \sigma\left(\phi_{T}^{2 \sigma}(x)-e^{2 i \rho_{T}} \phi^{2 \sigma}\left(x+y_{T}-y\right)\right) \\
-\sigma\left(\phi_{T}^{2 \sigma}(x)-e^{-2 i \rho_{T}} \phi^{2 \sigma}\left(x+y_{T}-y\right)\right) & -(\sigma+1)\left(\phi_{T}^{2 \sigma}(x)-\phi^{2 \sigma}\left(x+y_{T}-y\right)\right)
\end{array}\right),
\end{aligned}
$$

$$
\begin{gathered}
i \dot{\pi} \partial_{\pi} \tilde{W}_{T}(\pi):=\dot{v}\left(\begin{array}{c}
-\left(x+y_{T}\right) e^{i \rho_{T}} \phi\left(x+y_{T}-y\right) \\
\left(x+y_{T}\right) e^{-i \rho_{T}} \phi\left(x+y_{T}-y\right)
\end{array}\right)+\dot{\gamma}\left(\begin{array}{c}
-e^{i \rho_{T}} \phi\left(x+y_{T}-y\right) \\
e^{-i \rho_{T}} \phi\left(x+y_{T}-y\right)
\end{array}\right) \\
+i \dot{\alpha}\left(\begin{array}{c}
e^{i \rho_{T}} \partial_{\alpha} \phi\left(x+y_{T}-y\right) \\
e^{-i \rho_{T}} \partial_{\alpha} \phi\left(x+y_{T}-y\right)
\end{array}\right)+i \dot{D}\left(\begin{array}{c}
-e^{i \rho_{T}} \partial_{x} \phi\left(x+y_{T}-y\right) \\
-e^{-i \rho_{T}} \partial_{x} \phi\left(x+y_{T}-y\right)
\end{array}\right),
\end{gathered}
$$

$$
N_{T}(U, \pi):=\left(\begin{array}{c}
N_{1 T}(U, \pi) \\
N_{2 T}(U, \pi)
\end{array}\right)=\left(\begin{array}{c}
N_{1 T}(U, \pi) \\
-\overline{N_{1 T}(U, \pi)}
\end{array}\right),
$$


and

$$
\begin{aligned}
& N_{1 T}(U, \pi)=-\left|U_{1}+e^{i \rho_{T}} \phi\left(x+y_{T}-y\right)\right|^{2 \sigma}\left(U_{1}+e^{i \rho_{T}} \phi\left(x+y_{T}-y\right)\right) \\
& +\phi\left(x+y_{T}-y\right)^{2 \sigma+1} e^{i \rho_{T}}+(\sigma+1) \phi\left(x+y_{T}-y\right)^{2 \sigma} U_{1}+\sigma \phi\left(x+y_{T}-y\right)^{2 \sigma} e^{2 i \rho_{T}} U_{2} .
\end{aligned}
$$

Here $\rho_{T}=\rho_{T}(t, x)$ is as in Lemma 2.4 and $\phi\left(x+y_{T}-y\right)=\phi\left(x+y_{T}(t)-y(t), \alpha(t)\right)$. Finally, $Z$ is $\mathcal{J}$-invariant iff $U$ is $\mathcal{J}$-invariant, and $U$ is $\mathcal{J}$-invariant iff $U(0)$ is $\mathcal{J}$-invariant.

Proof. Throughout this proof we will adhere to the convention that $\phi=\phi(\cdot, \alpha(t))$ whereas $\phi_{T}=\phi\left(\cdot, \alpha_{T}\right)$. Write the equation (2.1) for $Z$ in the form

$$
i \partial_{t} Z-\mathcal{H}_{T} Z=F+\left(\mathcal{H}(\pi(t))-\mathcal{H}_{T}\right) Z
$$

where

$$
\mathcal{H}_{T}=\left(\begin{array}{cc}
-\partial_{x}^{2}-(\sigma+1)\left|W_{T}\right|^{2 \sigma} & -\sigma|W|^{2(\sigma-1)} W_{T}^{2} \\
\sigma|W|^{2(\sigma-1)} \bar{W}_{T}^{2} & \partial_{x}^{2}+(\sigma+1)\left|W_{T}\right|^{2 \sigma}
\end{array}\right)
$$

see (2.18) and (2.10). With $\mathcal{G}_{T}(t)$ defined as in (2.19), and with $p=-i \partial_{x}$,

$$
\begin{aligned}
i \frac{d}{d t} \mathcal{G}_{T}(t) f & =\left(\begin{array}{c}
i \dot{\mathfrak{g}}_{T}(t)^{-1} f_{1} \\
i \overline{\mathfrak{g}_{T}(t)^{-1} \overline{f_{2}}}
\end{array}\right)=\left(\begin{array}{c}
-\left(2 v_{T} p+\left|v_{T}\right|^{2}\right) \mathfrak{g}_{T}(t)^{-1} f_{1} \\
-\left(2 v_{T} p-\left|v_{T}\right|^{2}\right)
\end{array}\right) \\
& =\left(\begin{array}{cc}
-\left(2 v_{T} p+\left|v_{T}\right|^{2}\right) & 0 \\
0 & -\left(2 v_{T} p-\left|v_{T}\right|^{2}\right)
\end{array}\right) \mathcal{G}_{T}(t) f
\end{aligned}
$$

for any $f=\left(\begin{array}{l}f_{1} \\ f_{2}\end{array}\right)$. Furthermore,

$$
\begin{aligned}
& M_{T}(t) \mathcal{G}_{T}(t) \mathcal{H}_{T}\left(\begin{array}{l}
f_{1} \\
f_{2}
\end{array}\right) \\
& =\left(\begin{array}{cc}
e^{i \omega_{T}(t)} & 0 \\
0 & e^{-i \omega_{T}(t)}
\end{array}\right)\left(\begin{array}{c}
-\left(\partial_{x}^{2}+(\sigma+1) \phi_{T}^{2 \sigma}\right) \\
\left.\phi_{T}^{2 \sigma} e^{-2 i \theta_{T}\left(t, x+y_{T}\right)}\right) \frac{\mathfrak{g}_{T}(t)^{-1} f_{1}}{\mathfrak{g}_{T}(t)^{-1} \overline{f_{1}}}+\left(\partial_{T}^{2}+(\sigma+1) \phi_{T}^{2 \sigma} e^{2 i \theta_{T}\left(t, x+y_{T}\right)}\right) \\
\mathfrak{g}_{T}(t)^{-1} f_{2} \\
\mathfrak{g}_{T}(t)^{-1} \overline{f_{2}}
\end{array}\right) \\
& -\left(\begin{array}{cc}
e^{i \omega_{T}(t)} & 0 \\
0 & e^{-i \omega_{T}(t)}
\end{array}\right)\left(\begin{array}{cc}
-\left|v_{T}\right|^{2}+2 i v_{T} \partial_{x} & 0 \\
0 & \left|v_{T}\right|^{2}+2 i v_{T} \partial_{x}
\end{array}\right) \mathcal{G}_{T}(t)\left(\begin{array}{l}
f_{1} \\
f_{2}
\end{array}\right) \\
& =\left(\begin{array}{cc}
e^{i \omega_{T}(t)} & 0 \\
0 & e^{-i \omega_{T}(t)}
\end{array}\right) \\
& \times\left(\begin{array}{c}
\left.-\left(\partial_{x}^{2}+(\sigma+1) \phi_{T}^{2 \sigma}\right) \mathfrak{g}_{T}(t)^{-1} f_{1}-\sigma \phi_{T}^{2 \sigma} e^{2 i\left[\theta_{T}\left(t, x+y_{T}\right)-\left(t\left|v_{T}\right|^{2}+v_{T}\left(x+D_{T}\right)+\gamma_{T}\right)\right]}\right] \overline{\mathfrak{g}_{T}(t)^{-1} \overline{f_{2}}} \\
\sigma \phi_{T}^{2 \sigma} e^{-2 i\left[\theta_{T}\left(t, x+y_{T}\right)-\left(t\left|v_{T}\right|^{2}+v_{T}\left(x+D_{T}\right)+\gamma_{T}\right)\right]} \mathfrak{g}_{T}(t)^{-1} f_{1}+\left(\partial_{x}^{2}+(\sigma+1) \phi_{T}^{2 \sigma}\right)
\end{array}\right. \\
& -\left(\begin{array}{cc}
e^{i \omega_{T}(t)} & 0 \\
0 & e^{-i \omega_{T}(t)}
\end{array}\right)\left(\begin{array}{cc}
-\left|v_{T}\right|^{2}+2 i v_{T} \partial_{x} & 0 \\
0 & \left|v_{T}\right|^{2}+2 i v_{T} \partial_{x}
\end{array}\right) \mathcal{G}_{T}(t)\left(\begin{array}{l}
f_{1} \\
f_{2}
\end{array}\right) .
\end{aligned}
$$

Now

$$
\theta_{T}\left(t, x+y_{T}\right)-\left(t\left|v_{T}\right|^{2}+v_{T}\left(x+D_{T}\right)+\gamma_{T}\right)=t \alpha_{T}^{2}
$$


see (2.13). Hence, by the definition of $\omega(t)$ (and dropping the argument $t$ from $M_{T}$ and $\mathcal{G}_{T}$ for simplicity),

$$
\begin{aligned}
\underline{(2.27)}= & \left(\begin{array}{cc}
e^{i \omega_{T}(t)} & 0 \\
0 & e^{-i \omega_{T}(t)}
\end{array}\right)\left(\begin{array}{cc}
-\left(\partial_{x}^{2}+(\sigma+1) \phi_{T}^{2 \sigma}\right) & -\sigma \phi_{T}^{2 \sigma} e^{2 i t \alpha_{T}^{2}} \\
\sigma \phi_{T}^{2 \sigma} e^{-2 i t \alpha_{T}^{2}} & \partial_{x}^{2}+(\sigma+1) \phi_{T}^{2 \sigma}
\end{array}\right) \mathcal{G}_{T} f \\
& -\left(\begin{array}{cc}
e^{i \omega_{T}(t)} & 0 \\
0 & e^{-i \omega_{T}(t)}
\end{array}\right)\left(\begin{array}{cc}
-\left|v_{T}\right|^{2}-2 v_{T} p & 0 \\
0 & \left|v_{T}\right|^{2}-2 v_{T} p
\end{array}\right) \mathcal{G}_{T} f \\
= & \left(\begin{array}{cc}
-\partial_{x}^{2}-(\sigma+1) \phi_{T}^{2 \sigma} & -\sigma \phi_{T}^{2 \sigma} \\
\sigma \phi_{T}^{2 \sigma} & \partial_{x}^{2}+(\sigma+1) \phi_{T}^{2 \sigma}
\end{array}\right) M_{T} \mathcal{G}_{T} f \\
& -\left(\begin{array}{cc}
-\left|v_{T}\right|^{2}-2 v_{T} p & 0 \\
0 & \left|v_{T}\right|^{2}-2 v_{T} p
\end{array}\right) M_{T} \mathcal{G}_{T} f .
\end{aligned}
$$

Denote the first matrix operator in (2.28) by $\mathcal{H}_{\phi}$. Hence, in combination with (2.26) one concludes from (2.24) that

$$
\begin{aligned}
i \dot{U} & =i \dot{M}_{T} \mathcal{G}_{T} Z+i M_{T} \dot{\mathcal{G}}_{T} Z+M_{T} \mathcal{G}_{T} \mathcal{H}_{T}+M_{T} \mathcal{G}_{T}\left(F+\left(\mathcal{H}(\pi(t))-\mathcal{H}_{T}\right) Z\right) \\
= & \left(\begin{array}{cc}
-\dot{\omega}_{T} & 0 \\
0 & \dot{\omega}_{T}
\end{array}\right) M_{T} \mathcal{G}_{T} Z \\
& +\left(\begin{array}{cc}
-\left(2 v_{T} p+\left|v_{T}\right|^{2}\right) & 0 \\
0 & -\left(2 v_{T} p-\left|v_{T}\right|^{2}\right)
\end{array}\right) M_{T} \mathcal{G}_{T} Z+\mathcal{H}_{\phi} M_{T} \mathcal{G}_{T} Z \\
& +\left(\begin{array}{cc}
\left|v_{T}\right|^{2}+2 v_{T} p & 0 \\
0 & -\left|v_{T}\right|^{2}+2 v_{T} p
\end{array}\right) M_{T} \mathcal{G}_{T} Z+M_{T} \mathcal{G}_{T}\left(F+\left(\mathcal{H}(\pi(t))-\mathcal{H}_{T}\right) Z\right) \\
= & \left(\begin{array}{cc}
-\partial_{x}^{2}+\alpha_{T}^{2}-(\sigma+1) \phi_{T}^{2 \sigma} & -\sigma \phi_{T}^{2 \sigma} \\
\sigma \phi_{T}^{2 \sigma} & \partial_{x}^{2}-\alpha_{T}^{2}+(\sigma+1) \phi_{T}^{2 \sigma}
\end{array}\right) U(t) \\
& +M_{T} \mathcal{G}_{T}\left(F+\left(\mathcal{H}(\pi(t))-\mathcal{H}_{T}\right) \mathcal{G}_{T}^{-1} M_{T}^{-1} U\right) .
\end{aligned}
$$

It remains to compute the terms

$$
\begin{gathered}
-i \dot{\pi} \partial_{\pi} \tilde{W}_{T}(\pi)+N_{T}(U, \pi)=M_{T}(t) \mathcal{G}_{T}(t) F(t), \\
V_{T}=M_{T}(t) \mathcal{G}_{T}(t)\left(\mathcal{H}(\pi(t))-\mathcal{H}_{T}\right) \mathcal{G}_{T}(t)^{-1} M_{T}(t)^{-1} .
\end{gathered}
$$

In view of (2.1), one has

$$
\begin{aligned}
F= & -\dot{v}\left(\begin{array}{c}
-x e^{i \theta} \phi(x-y) \\
x e^{-i \theta} \phi(x-y)
\end{array}\right)-\dot{\gamma}\left(\begin{array}{c}
-e^{i \theta} \phi(x-y) \\
e^{-i \theta} \phi(x-y)
\end{array}\right) \\
& -i \dot{\alpha}\left(\begin{array}{c}
e^{i \theta} \partial_{\alpha} \phi(x-y) \\
e^{-i \theta} \partial_{\alpha} \phi(x-y)
\end{array}\right)-i \dot{D}\left(\begin{array}{c}
-e^{i \theta} \partial_{x} \phi(x-y) \\
-e^{-i \theta} \partial_{x} \phi(x-y)
\end{array}\right)+\left(\begin{array}{c}
N_{1}(Z, W) \\
N_{2}(Z, W)
\end{array}\right) .
\end{aligned}
$$

Now

$\theta\left(t, x+y_{T}\right)-\left(\alpha_{T}^{2} t+v_{T}\left(x+D_{T}\right)+t\left|v_{T}\right|^{2}+\gamma_{T}\right)=\theta\left(t, x+y_{T}\right)-\theta_{T}\left(t, x+y_{T}\right)=\rho_{T}(t, x) ;$

see (2.13) and Lemma 2.4. Thus, the first term of $M_{T} \mathcal{G}_{T} F$ is

$$
\begin{aligned}
& \dot{v}\left(\begin{array}{cc}
e^{i \omega_{T}} & 0 \\
0 & e^{-i \omega_{T}}
\end{array}\right)\left(\begin{array}{c}
-\left(x+y_{T}\right) e^{i \theta\left(t, x+y_{T}\right)} e^{-i\left(t\left|v_{T}\right|^{2}+v_{T}\left(x+D_{T}\right)+\gamma_{T}\right)} \phi\left(x+y_{T}-y\right) \\
\left(x+y_{T}\right) e^{-i \theta\left(t, x+y_{T}\right)} e^{i\left(t\left|v_{T}\right|^{2}+v_{T}\left(x+D_{T}\right)+\gamma_{T}\right)} \phi\left(x+y_{T}-y\right)
\end{array}\right) \\
& =\dot{v}\left(\begin{array}{c}
-\left(x+y_{T}\right) e^{i \rho_{T}(t, x)} \phi\left(x+y_{T}-y\right) \\
\left(x+y_{T}\right) e^{-i \rho_{T}(t, x)} \phi\left(x+y_{T}-y\right)
\end{array}\right) .
\end{aligned}
$$

This gives the $\dot{v}$ term in (2.22). The other terms involving $\dot{\alpha}, \dot{\gamma}$, and $\dot{D}$ are treated similarly, and we skip the details. The cubic term in (2.1) is also easily transformed, 
and it leads to the nonlinear term $N_{T}(U, \pi)$ in (2.23). We skip that calculation as well. Finally, it remains to transform $\mathcal{H}(\pi(t))-\mathcal{H}_{T}$. One has

$$
\begin{aligned}
& \mathcal{H}(\pi(t))-\mathcal{H}_{T} \\
& =\left(\begin{array}{cc}
(\sigma+1)\left(\phi_{T}^{2 \sigma}\left(\cdot-y_{T}\right)-\phi^{2 \sigma}(\cdot-y)\right) & \sigma\left(e^{2 i \theta_{T}} \phi_{T}^{2 \sigma}\left(\cdot-y_{T}\right)-e^{2 i \theta} \phi^{2 \sigma}(\cdot-y)\right) \\
-\sigma\left(e^{-2 i \theta} \phi^{2 \sigma}(\cdot-y)-e^{-2 i \theta_{T}} \phi_{T}^{2 \sigma}\left(\cdot-y_{T}\right)\right) & -(\sigma+1)\left(\phi_{T}^{2 \sigma}\left(\cdot-y_{T}\right)-\phi^{2 \sigma}(\cdot-y)\right)
\end{array}\right)
\end{aligned}
$$

where $\phi_{T}=\phi\left(\cdot-y_{T}(t), \alpha_{T}\right), \phi=\phi(\cdot-y(t), \alpha(t))$ for simplicity. It is easy to check that

$\mathcal{G}_{T}(t)\left(\mathcal{H}(\pi(t))-\mathcal{H}_{T}\right)=\left(\begin{array}{cc}(\sigma+1)\left(\phi_{T}^{2 \sigma}(x)-\phi^{2 \sigma}\left(x+y_{T}-y\right)\right) & * \\ -\sigma e^{-2 i t \alpha_{T}^{2}}\left(\phi_{T}^{2 \sigma}(x)-e^{-2 i \rho_{T}} \phi^{2 \sigma}\left(\cdot+y_{T}-y\right)\right) & *\end{array}\right) \mathcal{G}_{T}(t)$.

After conjugation by the matrix $M_{T}(t)$ this takes the desired form (2.21) and we are done. For the final statements concerning $\mathcal{J}$-invariance, observe first that the transformation (2.19) from $Z$ to $U$ preserves $\mathcal{J}$-invariance. Second, the equation (2.20) is $\mathcal{J}$-invariant, which shows that it suffices to assume the $\mathcal{J}$-invariance of $U(0)$ to guarantee it for all $t \geq 0$. To check the $\mathcal{J}$-invariance of (2.20), note that the right-hand side of (2.20) transforms like

$$
\mathcal{J}\left[-i \dot{\pi} \partial_{\pi} \tilde{W}_{T}(\pi)+N_{T}(U, \pi)+V_{T} U\right]=-\left[-i \dot{\pi} \partial_{\pi} \tilde{W}_{T}(\pi)+N_{T}(\mathcal{J} U, \pi)+V_{T} \mathcal{J} U\right],
$$

while the left-hand side transforms as follows:

$$
\begin{aligned}
& \mathcal{J}\left[i \dot{U}(t)+\left(\begin{array}{cc}
\partial_{x}^{2}+(\sigma+1) \phi_{T}^{2 \sigma}-\alpha_{T}^{2} & \sigma \phi_{T}^{2 \sigma} \\
-\sigma \phi_{T}^{2 \sigma} & -\partial_{x}^{2}-(\sigma+1) \phi_{T}^{2 \sigma}+\alpha_{T}^{2}
\end{array}\right) U\right] \\
& =-i \dot{J} U(t)-\left(\begin{array}{cc}
\partial_{x}^{2}+(\sigma+1) \phi_{T}^{2 \sigma}-\alpha_{T}^{2} & \sigma \phi_{T}^{2 \sigma} \\
-\sigma \phi_{T}^{2 \sigma} & -\partial_{x}^{2}-(\sigma+1) \phi_{T}^{2 \sigma}+\alpha_{T}^{2}
\end{array}\right) \mathcal{J} U .
\end{aligned}
$$

Combining these statements yields the desired $\mathcal{J}$-invariance of (2.20).

Next, we state a standard bound on the nonlinearity $N_{T}(U, \pi)$.

Lemma 2.7. The nonlinearity $N_{T}(U, \pi)$ from (2.23) satisfies

$$
\left|N_{T}(U, \pi)(x, t)\right| \leq C\left(|U(x, t)|^{2 \sigma+1}+|U(x, t)|^{2} \phi^{2 \sigma-1}\left(x+y_{T}-y\right)\right)
$$

for all $x, t \in \mathbb{R}$. Furthermore,

$$
\begin{aligned}
\left|\partial_{x} N_{T}(U, \pi)(x, t)\right| \leq & C\left(|U(x, t)|^{2 \sigma}+|U(x, t)| \phi^{2 \sigma-1}\left(x+y_{T}-y\right)\right)\left|\partial_{x} U(x, t)\right| \\
& +C\left(|U(x, t)|^{2 \sigma+1}+|U(x, t)|^{2} \phi^{2 \sigma-1}\left(x+y_{T}-y\right)\right) .
\end{aligned}
$$

Here $C$ only depends on $\sigma$.

Proof. Let

$$
F(z, w):=-|z+w|^{2 \sigma}(z+w)+|w|^{2 \sigma} w+(\sigma+1)|w|^{2 \sigma} z+\sigma|w|^{2(\sigma-1)} w^{2} \bar{z}
$$

for all $z, w \in \mathbb{C}$. Then $F(0, w)=0$ as well as $\partial_{z} F(0, w)=\partial_{\bar{z}} F(0, w)=0$. Hence,

$$
\sup _{|w|=1}|F(z, w)| \leq C\left(|z|^{2 \sigma+1}+|z|^{2}\right) .
$$

Rescaling implies that

$$
|F(z, w)| \leq C\left(|z|^{2 \sigma+1}+|z|^{2}|w|^{2 \sigma-1}\right) .
$$

As for the second statement, we use the bound

$$
\sup _{|w|=1}\left(\left|\partial_{z} F(z, w)\right|+\left|\partial_{\bar{z}} F(z, w)\right|\right) \leq C\left(|z|^{2 \sigma}+|z|\right),
$$


as well as

$$
\sup _{|w|=1}\left(\left|\partial_{w} F(z, w)\right|+\left|\partial_{\bar{w}} F(z, w)\right|\right) \leq C\left(|z|^{2 \sigma+1}+|z|^{2}\right) .
$$

Via the homogeneity, for all $w \neq 0$,

$$
\begin{aligned}
\left|\partial_{z} F(z, w)\right|+\left|\partial_{\bar{z}} F(z, w)\right| & \leq C\left(|z|^{2 \sigma}+|z||w|^{2 \sigma-1}\right), \\
\left|\partial_{w} F(z, w)\right|+\left|\partial_{\bar{w}} F(z, w)\right| & \leq C\left(|z|^{2 \sigma+1}|w|^{-1}+|z|^{2}|w|^{2(\sigma-1)}\right) .
\end{aligned}
$$

Thus, if $z=z(x)$ and $w=w(x)$, then

$\left|\partial_{x} F(z(x), w(x))\right| \leq C\left(|z|^{2 \sigma}+|z||w|^{2 \sigma-1}\right)\left|z_{x}\right|+C\left(|z|^{2 \sigma+1}|w|^{-1}+|z|^{2}|w|^{2(\sigma-1)}\right)\left|w_{x}\right|$,

which implies that

$$
\begin{aligned}
& \left|\partial_{x} N_{T}(U, \pi)(x, t)\right| \leq C\left(|U(x, t)|^{2 \sigma}+|U(x, t)| \phi^{2 \sigma-1}\left(x+y_{T}-y\right)\right)\left|\partial_{x} U(x, t)\right| \\
& \quad+C\left(|U(x, t)|^{2 \sigma+1}+|U(x, t)|^{2} \phi^{2 \sigma-1}\left(x+y_{T}-y\right)\right) \frac{\left|\partial_{x} \phi\left(x+y_{T}-y\right)\right|}{\phi\left(x+y_{T}-y\right)} .
\end{aligned}
$$

Since $\left\|\partial_{x} \phi / \phi\right\|_{\infty} \leq C$, the lemma follows.

\section{The LINEARIZED PROBLEM AND THE ROOT SPACES AT ZERO}

Recall that $\phi=\phi(\cdot, \alpha)$ is the ground state of $-\partial_{x}^{2} \phi+\alpha^{2} \phi=\phi^{2 \sigma+1}$. Define

$$
\mathcal{H}(\alpha):=\left(\begin{array}{cc}
-\partial_{x}^{2}-(\sigma+1) \phi^{2 \sigma}+\alpha^{2} & -\sigma \phi^{2 \sigma} \\
\sigma \phi^{2 \sigma} & \partial_{x}^{2}+(\sigma+1) \phi^{2 \sigma}-\alpha^{2}
\end{array}\right) .
$$

Hence the matrix operator on the left-hand side of (2.20) is equal to $-\mathcal{H}\left(\alpha_{T}\right)$; i.e., (2.20) can be rewritten as

$$
i \partial_{t} U-\mathcal{H}\left(\alpha_{T}\right) U=-i \dot{\pi} \partial_{\pi} \tilde{W}_{T}(\pi)+N_{T}(U, \pi)+V_{T} U
$$

or

$$
i \partial_{t} U-\mathcal{H}_{T}(t) U=-i \dot{\pi} \partial_{\pi} \tilde{W}_{T}(\pi)+N_{T}(U, \pi),
$$

where $\mathcal{H}_{T}(t):=\mathcal{H}\left(\alpha_{T}\right)+V_{T}(t)$.

In order to prove estimates on (2.20), we will need to have precise control on the evolution $e^{i t \mathcal{H}(\alpha)}$. Sections 5 9 deal with this issue. In particular, in Proposition 9.2 it is shown that the essential spectrum of $\mathcal{H}(\alpha)$ equals $\left(-\infty,-\alpha^{2}\right] \cup\left[\alpha^{2}, \infty\right)$ and that the discrete spectrum equals $\{0, \pm i \gamma(\alpha)\}$ with $\gamma=\gamma(\alpha)>0$. Here 0 is an eigenvalue of geometric multiplicity two and algebraic multiplicity four, whereas both $\pm i \gamma$ are simple eigenvalues (we are dealing with the supercritical case $\sigma>2$ ). In fact, $\mathcal{H}(\alpha) f^{ \pm}(\alpha)= \pm i \gamma f^{ \pm}(\alpha)$ where $f^{ \pm}(\alpha)$ are exponentially decaying (see Hundertmark and Lee [27]), and similarly $\mathcal{H}(\alpha)^{*} \tilde{f}^{ \pm}(\alpha)=\mp i \gamma \tilde{f}^{ \pm}(\alpha)$ (cf. also Corollary 9.3 below). In [55], Weinstein showed that the root spaces

$$
\mathcal{N}=\bigcup_{n=1}^{\infty} \operatorname{ker}\left(\mathcal{H}(\alpha)^{n}\right), \quad \mathcal{N}^{*}=\bigcup_{n=1}^{\infty} \operatorname{ker}\left(\left(\mathcal{H}(\alpha)^{*}\right)^{n}\right)
$$

of $\mathcal{H}(\alpha)$ and $\mathcal{H}^{*}(\alpha)$, respectively, are (with $\phi=\phi(\cdot, \alpha)$ )

$$
\begin{gathered}
\mathcal{N}=\mathcal{N}(\alpha)=\operatorname{span}\left\{\left(\begin{array}{c}
i \phi \\
-i \phi
\end{array}\right),\left(\begin{array}{c}
\partial_{\alpha} \phi \\
\partial_{\alpha} \phi
\end{array}\right),\left(\begin{array}{c}
\partial_{x} \phi \\
\partial_{x} \phi
\end{array}\right),\left(\begin{array}{c}
i x \phi \\
-i x \phi
\end{array}\right)\right\}, \\
\mathcal{N}^{*}=\mathcal{N}(\alpha)^{*}=\operatorname{span}\left\{\left(\begin{array}{c}
\phi \\
\phi
\end{array}\right),\left(\begin{array}{c}
i \partial_{\alpha} \phi \\
-i \partial_{\alpha} \phi
\end{array}\right),\left(\begin{array}{c}
i \partial_{x} \phi \\
-i \partial_{x} \phi
\end{array}\right),\left(\begin{array}{c}
x \phi \\
x \phi
\end{array}\right)\right\} .
\end{gathered}
$$


In particular, in (3.2) the kernels are the same starting with $n=2$. Let $P_{d}$ be the Riesz projection onto the discrete spectrum; i.e.,

$$
P_{d}=\frac{1}{2 \pi i} \oint_{\gamma}(z I-\mathcal{H})^{-1} d z
$$

where $\gamma$ is a simple closed curve that encloses the entire discrete spectrum of $\mathcal{H}$ and lies within the resolvent set (see Hislop and Sigal 26] for basic properties of these projcetions). Moreover, define $P_{s}=I-P_{d}$ ("s" here stands for "stable"). In Lemma 9.4 below we show that there is the direct and (skew) orthogonal splitting

$$
L^{2}(\mathbb{R}) \times L^{2}(\mathbb{R})=\mathcal{N}+\operatorname{span}\left\{f^{ \pm}(\alpha)\right\}+\left(\mathcal{N}^{*}+\operatorname{span}\left\{\tilde{f}^{ \pm}(\alpha)\right\}\right)^{\perp}
$$

Moreover, $P_{s}$ is exactly the projection onto the orthogonal complement on the right-hand side with kernel equal to the sum of the first two terms; i.e., it is the projection onto the orthogonal complement which is induced by this splitting.

The main estimates on $e^{i t \mathcal{H}} P_{s}$ are as follows (see Sections 66 8):

- $\sup _{t}\left\|e^{i t \mathcal{H}} P_{s} f\right\|_{2} \leq C\|f\|_{2}$ and $\sup _{t}\left\|e^{i t \mathcal{H}} P_{s} f\right\|_{H^{1}} \leq C\|f\|_{H^{1}}$,

- $\left\|\langle x\rangle e^{i t \mathcal{H}} P_{s} f\right\|_{2} \leq C\left(\langle t\rangle\|f\|_{H^{1}}+\|\langle x\rangle f\|_{2}\right)$,

- $\left\|\langle x\rangle^{-\theta} e^{i t \mathcal{H}} P_{s} f\right\|_{\infty} \leq C|t|^{-\frac{1}{2}-\theta}\left\|\langle x\rangle^{\theta} f\right\|_{1}$ for all $0 \leq \theta \leq 1$.

In order to apply these estimates to (2.20), we need to project $U$ onto $\operatorname{Ran}\left(P_{s}\right)$. Following common practice, see Soffer and Weinstein [49], [50] and Buslaev and Perelman [5], we will make an appropriate choice of the path $\pi(t)$ in order to insure that $U(t)$ is perpendicular to $\mathcal{N}^{*}$. However, for technical reasons it is necessary to impose an orthogonality condition onto a time-dependent family of functions rather than $\mathcal{N}^{*}$ itself. We introduce this family in the following definition. In view of Lemma 2.4] it approaches $\mathcal{N}^{*}$ in the limit $t \rightarrow T$.

Definition 3.1. Assume that $\pi$ is an admissible path and let $y, \theta$ be as in (1.5), (1.4), $y_{T}, \theta_{T}$ as in (2.10), and $\rho_{T}$ as in (2.11). With these functions, definet

$$
\begin{aligned}
& \tilde{\xi}_{1}(t):=\left(\begin{array}{c}
e^{i \rho_{T}} \phi\left(\cdot+\left(y_{T}-y\right)(t), \alpha(t)\right) \\
e^{-i \rho_{T}} \phi\left(\cdot+\left(y_{T}-y\right)(t), \alpha(t)\right)
\end{array}\right), \\
& \tilde{\xi}_{2}(t):=\left(\begin{array}{c}
i e^{i \rho_{T}} \partial_{\alpha} \phi\left(\cdot+\left(y_{T}-y\right)(t), \alpha(t)\right) \\
-i e^{-i \rho_{T}} \partial_{\alpha} \phi\left(\cdot+\left(y_{T}-y\right)(t), \alpha(t)\right)
\end{array}\right), \\
& \tilde{\xi}_{3}(t):=\left(\begin{array}{c}
e^{i \rho_{T}}\left(x+\left(y_{T}-y\right)(t)\right) \phi\left(\cdot+\left(y_{T}-y\right)(t), \alpha(t)\right) \\
e^{-i \rho_{T}}\left(x+\left(y_{T}-y\right)(t)\right) \phi\left(\cdot+\left(y_{T}-y\right)(t), \alpha(t)\right)
\end{array}\right), \\
& \tilde{\xi}_{4}(t):=\left(\begin{array}{c}
i e^{i \rho_{T}} \partial_{x} \phi\left(\cdot+\left(y_{T}-y\right)(t), \alpha(t)\right) \\
-i e^{-i \rho_{T}} \partial_{x} \phi\left(\cdot+\left(y_{T}-y\right)(t), \alpha(t)\right)
\end{array}\right) .
\end{aligned}
$$

\footnotetext{
${ }^{6}$ This notation is a bit inaccurate, since $\tilde{\xi}_{\ell}$ depends on both $T$ and the path $\pi$ chosen. We will later explicitly denote the path dependence by $\xi_{\ell}(\pi)$ and imply a time $T$ explicitly chosen for each path $\pi$.
} 
We similarly introduce the notation

$$
\begin{aligned}
\xi_{1}(t) & :=\left(\begin{array}{c}
e^{i \theta(t, x)} \phi(\cdot-y(t), \alpha(t)) \\
e^{-i \theta(t, x)} \phi(\cdot-y(t), \alpha(t))
\end{array}\right), \\
\xi_{2}(t) & :=\left(\begin{array}{c}
i e^{i \theta(t, x)} \partial_{\alpha} \phi(\cdot-y(t), \alpha(t)) \\
-i e^{-i \theta(t, x)} \partial_{\alpha} \phi(\cdot-y(t), \alpha(t))
\end{array}\right), \\
\xi_{3}(t) & :=\left(\begin{array}{c}
e^{i \theta(t, x)}(x-y(t)) \phi(\cdot-y(t), \alpha(t)) \\
e^{-i \theta(t, x)}(x-y(t)) \phi(\cdot-y(t), \alpha(t))
\end{array}\right), \\
\xi_{4}(t) & :=\left(\begin{array}{c}
i e^{i \theta(t, x)} \partial_{x} \phi(\cdot-y(t), \alpha(t)) \\
-i e^{-i \theta(t, x)} \partial_{x} \phi(\cdot-y(t), \alpha(t))
\end{array}\right) .
\end{aligned}
$$

We also introduce other families $\left\{\tilde{\eta}_{j}\right\}_{j=1}^{4}$ and $\left\{\eta_{j}\right\}_{j=1}^{4}$ by

$$
\tilde{\eta}_{j}=\left(\begin{array}{cc}
-i & 0 \\
0 & i
\end{array}\right) \tilde{\xi}_{j}, \eta_{j}=\left(\begin{array}{cc}
-i & 0 \\
0 & i
\end{array}\right) \xi_{j}, \text { for any } 1 \leq j \leq 4 .
$$

By inspection, $\mathcal{J} \tilde{\xi}_{j}=\tilde{\xi}_{j}, \mathcal{J} \xi_{j}=\xi_{j}$ for $1 \leq j \leq 4$ and we chose $\tilde{\eta}_{j}, \eta_{j}$ in such a way that $\mathcal{J} \tilde{\eta}_{j}=\tilde{\eta}_{j}, \mathcal{J} \eta_{j}=\eta_{j}$ for each $j$. Clearly, while the $\tilde{\xi}_{j}, \xi_{j}$ correspond to $\mathcal{H}^{*}$; the $\tilde{\eta}_{j}, \eta_{j}$ correspond to $\mathcal{H}$, see (3.3) and (3.4). Next, we modify the $\gamma$ parameter.

Lemma 3.2. Let $\pi(t)$ be an admissible path as in Definition 2.3. Set

$$
\dot{\tilde{\gamma}}(t):=\dot{\gamma}(t)+\dot{v}(t) y(t)
$$

and $\tilde{\gamma}(0):=0$, i.e.,

$$
\tilde{\gamma}(t):=\int_{0}^{t}[\dot{\gamma}(s)+\dot{v}(s) y(s)] d s .
$$

Then the function $\dot{\pi} \partial_{\pi} \tilde{W}_{T}(\pi)$ on the right-hand side of (2.20) satisfies

$$
\dot{\pi} \partial_{\pi} \tilde{W}_{T}(\pi)=-\dot{D} \tilde{\eta}_{4}-\dot{v} \tilde{\eta}_{3}+\dot{\alpha} \tilde{\eta}_{2}-\dot{\tilde{\gamma}} \tilde{\eta}_{1}
$$

where the functions $\left\{\tilde{\eta}_{j}\right\}_{j=1}^{4}$ are as in (3.6). Similarly, we have (see (2.1))

$$
\dot{\pi} \partial_{\pi} W=-\dot{D} \eta_{4}-\dot{v} \eta_{3}+\dot{\alpha} \eta_{2}-\dot{\tilde{\gamma}} \eta_{1} .
$$

Proof. By inspection.

The following lemma records some useful facts about the two families in Definition 3.1

Lemma 3.3. Let $\phi=\phi(\cdot, \alpha(t))$ be the ground state of (1.2) and let $\left\{\tilde{\xi}_{j}\right\}_{j=1}^{4}$ and $\left\{\tilde{\eta}_{j}\right\}_{j=1}^{4}$ be as in Definition 3.1. Then

$$
\begin{aligned}
& \left\langle\tilde{\xi}_{1}, \tilde{\eta}_{j}\right\rangle=2\left\langle\partial_{\alpha} \phi, \phi\right\rangle \text { if } j=2 \text { and }=0 \text { else, } \\
& \left\langle\tilde{\xi}_{2}, \tilde{\eta}_{j}\right\rangle=-2\left\langle\partial_{\alpha} \phi, \phi\right\rangle \quad \text { if } j=1 \text { and }=0 \text { else, } \\
& \left\langle\tilde{\xi}_{3}, \tilde{\eta}_{j}\right\rangle=-\langle\phi, \phi\rangle \quad \text { if } j=4 \text { and }=0 \text { else, } \\
& \left\langle\tilde{\xi}_{4}, \tilde{\eta}_{j}\right\rangle=\langle\phi, \phi\rangle \text { if } j=3 \text { and }=0 \text { else. }
\end{aligned}
$$

Here $\partial_{\alpha}\langle\phi, \phi\rangle=2\left\langle\partial_{\alpha} \phi, \phi\right\rangle=\left(2 \sigma^{-1}-1\right) \alpha^{-1}\|\phi\|_{2}^{2}$.

Proof. A simple calculation. 
We can now derive the usual modulation equations for the admissible path $\pi$ under the orthogonality condition

$$
\left\langle U(t), \tilde{\xi}_{j}(t)\right\rangle=0
$$

for all $t \geq 0,1 \leq j \leq 4$.

Lemma 3.4. Assume that $\pi$ is an admissible path and that $U$ is an $H^{1}$ solution of (2.20) with an initial condition $U(0)$ which satisfies the orthogonality condition (3.8) at time $t=0$. Then $U$ satisfies the orthogonality assumptions (3.8) for all times iff $\pi$ satisfies the following modulation equations (with $\phi=\phi(\cdot, \alpha(t))$ )

$$
\begin{aligned}
\dot{\alpha}\left(2 \sigma^{-1}-1\right) \alpha^{-1}\|\phi\|_{2}^{2} & =\left\langle U, \dot{\tilde{\pi}} \tilde{\mathcal{S}}_{1}\left(.+y_{T}-y\right)\right\rangle+\left\langle i N_{T}(U, \pi), \tilde{\xi}_{1}\right\rangle, \\
\dot{\tilde{\gamma}}\left(2 \sigma^{-1}-1\right) \alpha^{-1}\|\phi\|_{2}^{2} & =\left\langle U, \dot{\tilde{\pi}} \tilde{\mathcal{S}}_{2}\left(.+y_{T}-y\right)\right\rangle+\left\langle i N_{T}(U, \pi), \tilde{\xi}_{2}\right\rangle, \\
\dot{D}\|\phi\|_{2}^{2} & =\left\langle U, \dot{\tilde{\pi}} \tilde{\mathcal{S}}_{3}\left(.+y_{T}-y\right)\right\rangle+\left\langle i N_{T}(U, \pi), \tilde{\xi}_{3}\right\rangle, \\
-\dot{v}\|\phi\|_{2}^{2} & =\left\langle U, \dot{\tilde{\pi}} \tilde{\mathcal{S}}_{4}\left(.+y_{T}-y\right)\right\rangle+\left\langle i N_{T}(U, \pi), \tilde{\xi}_{4}\right\rangle .
\end{aligned}
$$

In these formulae we denote by $\dot{\tilde{\pi}} \tilde{\mathcal{S}}_{\ell}\left(.+y_{T}-y\right)$ a linear combination of four rapidly decaying smooth functions with coefficients $\dot{\tilde{\pi}}$ and centered at $\left(y_{T}-y\right)(t)$. The righthand side is real-valued, consisting of scalar products of $\mathcal{J}$-invariant vectors. We denote (as before) $\tilde{\pi}(t)=(\tilde{\gamma}(t), v(t), D(t), \alpha(t))$.

Proof. The orthogonality conditions (3.8) are equivalent to the conditions $\left\langle Z, \xi_{\ell}\right\rangle=$ $0, \ell=1,2,3,4$. Observe that assuming this, we have an equality of the form

$$
\left\langle\left[i \partial_{t}-\mathcal{H}(\pi(t))\right] Z, \xi_{\ell}\right\rangle=\left\langle Z, \dot{\tilde{\pi}} \mathcal{S}_{\ell}(.-y(t))\right\rangle
$$

for suitable rapidly decaying functions $\mathcal{S}_{\ell}(\cdot)$. More precisely, without assuming the orthogonality, we have for suitable $\lambda_{\ell} \in \mathbb{C}$

$$
\left\langle\left[i \partial_{t}-\mathcal{H}(\pi(t))\right] Z, \xi_{\ell}\right\rangle=\left\langle Z, \dot{\tilde{\pi}} \mathcal{S}_{\ell}(.-y(t))\right\rangle+\lambda_{\ell}\left\langle Z, \xi_{\ell}\right\rangle+i \partial_{t}\left\langle Z, \xi_{\ell}\right\rangle .
$$

The statement of the lemma follows from this, the fact that solutions to first order linear ODE vanish identically if they vanish at one point, and the preceding lemmata.

Remark 3.5. The proof reveals that one may cast this system in the $Z$-picture schematically as follows:

$$
\left\langle-i \dot{\tilde{\pi}} \partial_{\pi} W(\pi), \xi_{\ell}(\pi)\right\rangle=\left\langle Z(t), i \dot{\tilde{\pi}} \mathcal{S}_{\ell}(\pi)(t)\right\rangle-\left\langle N(Z, \pi), \xi_{\ell}(t)\right\rangle .
$$

Equivalently, in the $U$-picture, this can be written

$$
\left\langle-i \dot{\tilde{\pi}} \partial_{\pi} W(\pi), \xi_{\ell}(\pi)\right\rangle=\left\langle U(t), i \dot{\tilde{\pi}} \tilde{\mathcal{S}}_{\ell}\left(.+y_{T}-y\right)(t)\right\rangle-\left\langle N_{T}(U, \pi), \tilde{\xi}_{\ell}(t)\right\rangle .
$$

\section{Constructing the solution: The iteration scheme}

According to Lemma 2.1, in order to solve the NLS equation (1.1) with $\psi(t)=$ $W(t)+R(t)$, we need to find an admissible path $\pi(t)$ as well as a function

$$
Z \in C\left([0, \infty), H^{1}(\mathbb{R}) \times H^{1}(\mathbb{R})\right) \cap C^{1}\left([0, \infty), H^{-1}(\mathbb{R}) \times H^{-1}(\mathbb{R})\right)
$$

so that $Z(t)$ is $\mathcal{J}$-invariant and such that $(\pi(t), Z(t))$ together satisfy (2.1). This will be accomplished by means of an iteration argument. To explain it, we will need to deal with several paths simultaneously. Therefore, our notation will need to indicate relative to which paths Galilei transforms, root spaces, etc., are defined. For example, $\mathcal{G}_{T}(\pi)(t)$ will mean the (vector) Galilei transform from (2.19) defined 
in terms of $\pi$, and $\left\{\tilde{\xi}_{j}(\pi)(t)\right\}_{j=1}^{4}$ will be the set of functions from Definition 3.1 which are obtained from $\pi$ together with a time $T$ specified explicitly in conjunction with $\pi$. The iteration scheme is based on the linearized equation (2.1). In principle, we want a suitable bounded subset of some Banach space $\tilde{X}_{*}$, containing $(Z, \pi)$ (defined globally in time), such that given some $\left(\pi^{(0)}, Z^{(0)}\right) \in \tilde{X}_{*}$ with $Z^{(0)}=\left(\begin{array}{c}R^{(0)} \\ \bar{R}^{(0)}\end{array}\right)$, we can solve for

$$
\begin{aligned}
& i \partial_{t} Z(t)+\left(\begin{array}{cc}
\partial_{x}^{2}+(\sigma+1)\left|W\left(\pi^{(0)}\right)\right|^{2} & \sigma W^{2}\left(\pi^{(0)}\right) \\
-\sigma \bar{W}^{2}\left(\pi^{(0)}\right) & -\partial_{x}^{2}-(\sigma+1)\left|W\left(\pi^{(0)}\right)\right|^{2}
\end{array}\right) Z(t) \\
& =-i \dot{\pi} \partial_{\pi} W\left(\pi^{(0)}\right)+N\left(Z^{(0)}, \pi^{(0)}\right) .
\end{aligned}
$$

The vector $\dot{\tilde{\pi}}$ is determined by means of the modulation equations. The initial datum $Z(0)$ should be chosen in such a fashion that the solution $Z(t)$ does not grow in time, i.e., such that the exponentially growing mode remain controlled; see below. The initial datum $\pi(0)$ is a fixed constant. Then we need to show that $(Z, \pi)$ again lies in the same subset of $\tilde{X}_{*}$. Unfortunately, this straightforward approach runs into severe difficulties due to the fact that our norms (defining the underlying Banach space) contain weights centered around the usual $y$-curve determined by the path $\pi^{(0)}$, and $\pi$ diverges from $\pi^{(0)}$ at infinity, leading to an incompatible norm. In particular, there is no absolute $\tilde{X}_{*}$ we can work with, but only relative versions of the form $\tilde{X}_{*}\left(\pi^{(0)}\right)$, depending on the given path. Our way out of this consists in adapting the time intervals on which the iterates are constructed. More precisely, we shall solve the above equation on progressively longer intervals, which are chosen in such a fashion that the paths differ very little on them. In particular, the weighted norms are all compatible on such intervals. The details of the iterative construction are rather involved and we now present them:

Definition 4.1. We let $X_{*}$ be the subset of the function space:

$$
\begin{aligned}
X:=\left\{(\pi, U) \in \operatorname{Lip}\left([0, \infty), \mathbb{R}^{4}\right) \times[\right. & L^{\infty}\left((0, \infty), H^{1}(\mathbb{R}) \times H^{1}(\mathbb{R})\right) \\
& \left.\left.\cap L_{\mathrm{loc}}^{\infty}((0, \infty), Y \times Y)\right] \mid \pi(0)=\left(0,0,0, \alpha_{0}\right)\right\}
\end{aligned}
$$

where $Y=\left\{f \in H^{1}(\mathbb{R}) \mid\langle x\rangle f \in L^{2}, \partial_{x} f \in L^{q}(\mathbb{R})\right\}$ for a very large number 7 , for which the following norm is $<\infty$ :

$$
\begin{array}{r}
\|(\pi, U)\|_{X_{*}}:=\sup _{0 \leq t<\infty}\left\{\langle t\rangle^{2+\epsilon}[|\dot{\alpha}(t)|+|\dot{v}(t)|+|\dot{\tilde{\gamma}}(t)|+|\dot{D}(t)|]+\|U(t)\|_{2}\right. \\
+\left\|\partial_{x} U(t)\right\|_{2}+\sup _{0 \leq \theta \leq 1}\langle t\rangle^{-\theta}\left\|\langle x\rangle^{\theta} U(t)\right\|_{2}+\sup _{0 \leq \theta \leq \frac{1}{2}+\epsilon}\langle t\rangle^{\frac{1}{2}+\theta}\left\|\langle x\rangle^{-\theta} U(t)\right\|_{\infty} \\
\left.+\langle t\rangle^{1+\varepsilon}\left\|\langle x\rangle^{-\frac{1}{2}-2 \varepsilon} \partial_{x} U(t)\right\|_{q}\right\} .
\end{array}
$$

We define $X_{*}([0, T])$ analogously, replacing $\infty$ by $T$. We also introduce timelocalized versions of this norm, as follows:

$$
\|(\pi, U)\|_{X_{*}([0, T])}:=\inf _{\left.(\tilde{\pi}, \tilde{U})\right|_{[0, T]}=(\pi, U)}\|(\tilde{\pi}, \tilde{U})\|_{X_{*}} \cdot
$$

In the last line, $(\tilde{\pi}, \tilde{U})$ are to be in $X$. Finally, we introduce $\|(\pi, Z)\|_{\tilde{X}_{*}\left(\pi^{(0)}\right)}$. The definition of the latter is the same as for $\|\cdot\|_{X_{*}}$, except that we replace $\langle x\rangle$ be

\footnotetext{
${ }^{7}$ It will be seen that $q \rightarrow \infty$ as $\sigma \rightarrow 2$.
} 
$\left\langle x-y^{(0)}(t)\right\rangle$. Here the quantity $y^{(0)}(t)$ is given by (1.5) with respect to the path $\pi^{(0)}$. Time-localized versions of this norm are defined as before.

With the norm (4.1) the space $X_{*}$ becomes a Banach space. With these tools we can now detail the iterative step and the a priori estimates: we shall again use the notation

$$
\dot{\tilde{\pi}}=(\dot{\tilde{\gamma}}, \dot{v}, \dot{D}, \dot{\alpha}), \dot{\tilde{\gamma}}=\dot{\gamma}+\dot{v} y
$$

Also, we shall use the notation

$$
\tilde{\pi}(t)=\int_{0}^{t} \dot{\tilde{\pi}}(s) d s+\left(0,0,0, \alpha_{0}\right) .
$$

Theorem 4.2. Let $T_{i}=i+\delta^{-1}$, where $\delta>0$ is as in the preceding definition, $i \in \mathbf{Z}_{\geq 0}$. There exists $\delta_{0}>0$ such that if $0 \leq \delta<\delta_{0}$, there exist positive numbers $A, C$ with the following properties: Assume that for $i \geq 1$, we are given $\left(\pi^{(j)}, Z^{(j)}\right) \in$ $X_{*}\left(\left[0, T_{i}\right]\right), 0 \leq j \leq i-1$, all $Z^{(j)} \mathcal{J}$-invariant, with the properties

$$
\left\|\left(\pi^{j}, Z^{(j)}\right)\right\|_{\tilde{X}_{*}\left(\pi^{(j-1)}\right)\left(\left[0, T_{j}\right]\right)}<C \delta, \max _{1 \leq j \leq i-1} T_{j} \sup _{t \in\left[0, T_{j}\right]}\left|\tilde{\pi}^{(j)}-\tilde{\pi}^{(j-1)}\right|(t)<A .
$$

Also, assume that $Z^{(j)}$ is constant past time $t=T_{j}$ and that $\pi^{(j)}$ is a straight line past $t=T_{j}$. Then given $R_{0}$ as in Theorem 1.1, there exists a canonical procedure for determining $\mathcal{J}$-invariant initial dat $8 Z^{(i)}(0)$ satisfying $\left\langle Z^{(i)}(0), \xi_{\ell}\left(\pi^{(i-1)}\right)\right\rangle=0$, such that the following conclusion applies: the combined system

$$
\begin{array}{ll}
i \partial_{t} Z^{(i)}(t)+\left(\begin{array}{cc}
\partial_{x}^{2}+(\sigma+1)\left|W\left(\pi^{(i-1)}\right)\right|^{2 \sigma} & \sigma|W|^{2 \sigma-2}\left(\pi^{(i-1)}\right) W^{2}\left(\pi^{(i-1)}\right) \\
-\sigma|W|^{2 \sigma-2}\left(\pi^{(i-1)}\right) W^{2}\left(\pi^{(i-1)}\right) & -\partial_{x}^{2}-(\sigma+1)\left|W\left(\pi^{(i-1)}\right)\right|^{2 \sigma}
\end{array}\right) Z^{(i)}(t) \\
=-i \dot{\pi}^{(i)} \partial_{\pi} W\left(\pi^{(i-1)}\right)+N\left(Z^{(i-1)}, \pi^{(i-1)}\right),
\end{array}
$$

$\left.Z^{(i)}(t)\right|_{t=0}=Z^{(i)}(0)$,

$$
\begin{aligned}
\left\langle i \dot{\pi}^{(i)} \partial_{\pi} W\left(\pi^{(i-1)}\right), \xi_{\ell}\left(\pi^{(i-1)}\right)\right\rangle=-\langle & \left.Z^{(i)}(t), i \dot{\tilde{\pi}}^{(i-1)} \mathcal{S}_{\ell}\left(\pi^{(i-1)}\right)(t)\right\rangle \\
& +\left\langle N\left(Z^{(i-1)}, \pi^{(i-1)}\right), \xi_{\ell}\left(\pi^{(i-1)}(t)\right)\right\rangle,
\end{aligned}
$$

$\pi^{(i)}(0)=\left(0,0,0, \alpha_{0}\right)$

has a solution on $\left[0, T_{i}\right]$ satisfying the inequalities

$$
\left\|\left(\pi^{(i)}, Z^{(i)}\right)\right\|_{\tilde{X}_{*}\left(\pi^{(i-1)}\right)\left(\left[0, T_{i}\right]\right)}<C \delta, T_{i} \sup _{t \in\left[0, T_{i}\right]}\left|\tilde{\pi}^{(i)}-\tilde{\pi}^{(i-1)}\right|(t)<A .
$$

Also, $Z^{(i)}$ is $\mathcal{J}$-invariant, and $\delta$ can be made small independently of $A, C$.

In light of the theorem, we can make the following

Definition 4.3. Let $\delta_{0}>0$ and $A, C$ be as in the preceding theorem. The iterates $Z^{(i)}$ are defined as follows: put

$$
Z^{(0)}:=\left(\frac{R_{0}}{R_{0}}\right), \quad \pi^{(0)}:=\left(0,0,0, \alpha_{0}\right)
$$

\footnotetext{
${ }^{8}$ Of course, these initial data are not just close to $\left(\frac{R_{0}}{R_{0}}\right)$, but they are a uniquely defined perturbation thereof.
} 
Then determine $\left(Z^{(i)}, \pi^{(i)}\right), i \geq 1$, from the preceding theorem: given $\left(\pi^{(0)}, Z^{(0)}\right)$, $\ldots,\left(\pi^{(i-1)}, Z^{(i-1)}\right)$, one constructs $\left(\pi^{(i)}, Z^{(i)}\right)$ on $\left[0, T_{i}\right]$ and extends $Z^{(i)}$ beyond $T_{i}$ as a constant and $\pi^{(i)}$ as a straight line.

We now prove the theorem.

Proof. In order to avoid confusion, we shall stick to the following conventions: we let $\tilde{\xi}_{\ell}\left(\pi^{(i)}\right)$, etc., denote the functions defined in Definition 3.1, with the translations $\left(y_{T}-y\right)(t)$ replaced by $\left(y_{T_{i}}^{(i)}-y^{(i)}\right)(t)$, etc., and time $T_{i}$ as above. Also, we denote the basis of the root space of the operator

$$
\left(\begin{array}{cc}
\triangle+(\sigma+1) \phi\left(x, \alpha_{T_{i}}^{(i-1)}\right)^{2 \sigma}-\left(\alpha_{T_{i}}^{(i-1)}\right)^{2} & \sigma \phi\left(x, \alpha_{T_{i}}^{(i-1)}\right)^{2 \sigma} \\
-\sigma \phi\left(x, \alpha_{T_{i}}^{(i-1)}\right)^{2 \sigma} & -\triangle-(\sigma+1) \phi\left(x, \alpha_{T_{i}}^{(i-1)}\right)^{2 \sigma}+\left(\alpha_{T_{i}}^{(i-1)}\right)^{2}
\end{array}\right)
$$

by $\tilde{\eta}_{\ell}\left(\alpha_{T_{i}}^{(i-1)}\right)$ and let eigenvectors corresponding to the imaginary eigenvalues $\pm i \gamma\left(\alpha_{T_{i}}^{(i-1)}\right)$ be $f^{ \pm}\left(\alpha_{T_{i}}^{(i-1)}\right)$, both of which are chosen to be $\mathcal{J}$-invariant. We shall make the following ansatz for $Z^{(i)}(0)$ :

$$
Z^{(i)}(0)=\left(\frac{R_{0}}{R_{0}}\right)+h^{(i)} f^{+}\left(\alpha_{T_{i}}^{(i-1)}\right)+\sum_{j=1}^{4} a_{j}^{(i)} \tilde{\eta}_{j}\left(\alpha_{T_{i}}^{(i-1)}\right), \quad h^{(i)}, a_{j}^{(i)} \in \mathbb{R} .
$$

As stated in the theorem, we want this to satisfy the orthogonality relations

$$
\left\langle Z^{(i)}(0), \xi_{\ell}\left(\pi^{(i-1)}(0)\right)\right\rangle=0 .
$$

The assumptions in the theorem imply in particular that $\left|\tilde{\eta}_{j}\left(\alpha_{T_{i}}^{(i-1)}\right)-\tilde{\eta}\left(\alpha_{0}\right)\right| \lesssim \delta$. This entails that for $\delta$ sufficiently small, we may uniquely solve the system

$$
\left\langle\left(\frac{R_{0}}{R_{0}}\right)+h^{(i)} f^{+}\left(\alpha_{T_{i}}^{(i-1)}\right)+\sum_{j=1}^{4} a_{j}^{(i)} \tilde{\eta}_{j}\left(\alpha_{T_{i}}^{(i-1)}\right), \xi_{\ell}\left(\pi^{(i-1)}(0)\right)\right\rangle=0
$$

for the $a_{j}^{(i)}$, for given $h^{(i)} \in \mathbb{R}$ and fixed $R_{0}$ as in Theorem 1.1. Moreover, the fact that both $\tilde{\eta}_{j}\left(\alpha_{T_{i}}^{(i-1)}\right), \xi_{\ell}\left(\pi^{(i-1)}(0)\right)$ are $\mathcal{J}$-invariant implies that $a_{j}^{(i)}\left(h^{(i)}, \alpha_{T_{i}}^{(i-1)}\right) \in \mathbb{R}$ for $h^{(i)} \in \mathbb{R}$. We shall then determine $h^{(i)}$ in such a fashion that the corresponding solution $Z^{(i)}(t)$ does not grow with time. In order to carry out estimates, we apply a gauge transformation to (4.2). Specifically, we put

$$
U^{(i)}=M_{T_{i}}\left(\pi^{(i-1)}\right) \mathcal{G}_{T_{i}}\left(\pi^{(i-1)}\right) Z^{(i)},
$$

where we have

$$
\begin{gathered}
M_{T_{i}}\left(\pi^{(i-1)}\right)(t)=\left(\begin{array}{cc}
e^{i \alpha^{(i-1)}\left(T_{i}\right)^{2} t} & 0 \\
0 & e^{-i \alpha^{(i-1)}\left(T_{i}\right)^{2} t}
\end{array}\right), \\
\mathcal{G}_{T_{i}}\left(\pi^{(i-1)}\right)(t, x)=\left(\begin{array}{c}
e^{-i\left(\gamma_{T_{i}}^{(i-1)}+v_{T_{i}}^{(i-1)} D_{T_{i}}^{(i-1)}+v_{T_{i}}^{(i-1)} x+t\left(v_{T_{i}}^{(i-1)}\right)^{2}\right)} e^{i\left(2 t v_{T_{i}}^{(i-1)}+D_{T_{i}}^{(i-1)}\right) p} \\
e^{-i\left(\gamma_{T_{i}}^{(i-1)}+v_{T_{i}}^{(i-1)} D_{T_{i}}^{(i-1)}+v_{T_{i}}^{(i-1)} x+t\left(v_{T_{i}}^{(i-1)}\right)^{2}\right)} e^{i\left(2 t v_{T_{i}}^{(i-1)}+D_{T_{i}}^{(i-1)}\right) p}
\end{array}\right) .
\end{gathered}
$$


The notation here is the same as in Section 2. We apply the same procedure to the $Z^{(j)}$, thereby introducing quantities $U^{(j)}, j \leq i-1$. Proceeding as in Section 2, we derive the following equation in the gauged picture:

$$
\begin{aligned}
& i \partial_{t} U^{(i)}-\mathcal{H}\left(\alpha_{T_{i}}^{(i-1)}\right) U^{(i)} \\
& \begin{aligned}
=M_{T_{i}}\left(\pi^{(i-1)}\right) \mathcal{G}_{T_{i}}\left(\pi^{(i-1)}\right)\left[-i \dot{\pi}^{(i)}\right. & \partial_{\pi} W\left(\pi^{(i-1)}\right)+N\left(Z^{(i-1)}, \pi^{(i-1)}\right) \\
& \left.+\left(\mathcal{H}\left(\pi^{(i-1)}(t)\right)-\mathcal{H}\left(\pi_{T_{i}}^{(i-1)}\right)(t)\right) Z^{(i)}\right]
\end{aligned}
\end{aligned}
$$

Here $\mathcal{H}\left(\pi_{T_{i}}^{(i-1)}(t)\right)$ is defined as $\mathcal{H}\left(\pi^{(i-1)}(t)\right)$ with the path $y^{(i-1)}(t)$ replaced by the straight line path given by $2 t v_{T_{i}}^{(i-1)}+D_{T_{i}}^{(i-1)}$. Written out, the following equation results:

$$
\begin{aligned}
& i \dot{U}^{(i)}(t)+\left(\begin{array}{cc}
\partial_{x}^{2}+(\sigma+1) \phi_{T_{i}}^{2 \sigma}-\left(\alpha_{T_{i}}^{(i-1)}\right)^{2} & \sigma \phi_{T_{i}}^{2 \sigma} \\
-\sigma \phi_{T_{i}}^{2 \sigma} & -\partial_{x}^{2}-(\sigma+1) \phi_{T_{i}}^{2 \sigma}+\left(\alpha_{T_{i}}^{(i-1)}\right)^{2}
\end{array}\right) U^{(i)} \\
& =-i \dot{\pi}^{(i)} \partial_{\pi} \tilde{W}_{T_{i}}\left(\pi^{(i-1)}\right) \\
& +N_{T_{i}}\left(M_{T_{i-1}}\left(\pi^{(i-1)}\right) \mathcal{G}_{T_{i-1}}\left(\pi^{(i-1)}\right) \mathcal{G}_{T_{i-2}}\left(\pi^{(i-2)}\right)^{-1} M_{T_{i-2}}\left(\pi^{(i-2)}\right)^{-1} U^{(i-1)}, \pi^{(i-1)}\right) \\
& +V^{(i-1)} U^{(i)} .
\end{aligned}
$$

We use the abbreviation $\phi_{T_{i}}=\phi\left(., \alpha_{T_{i}}^{(i-1)}\right)$, as well as the following:

$$
\begin{aligned}
& V^{(i-1)}=V_{T_{i}}\left(\pi^{(i-1)}\right) \\
& =\left(\begin{array}{cc}
(\sigma+1)\left(\phi_{T_{i}}^{2 \sigma}-\phi^{2 \sigma}\left(\cdot+y_{T_{i}}^{(i-1)}-y^{(i-1)}\right)\right) & \sigma\left(\phi_{T_{i}}^{2 \sigma}-e^{2 i \rho_{T_{i}}^{(i-1)}} \phi^{2 \sigma}\left(\cdot+y_{T_{i}}^{(i-1)}-y^{(i-1)}\right)\right) \\
-\sigma\left(\phi_{T_{i}}^{2 \sigma}-e^{-2 i \rho_{T_{i}}^{(i-1)}} \phi^{2 \sigma}\left(\cdot+y_{T_{i}}^{(i-1)}-y^{(i-1)}\right)\right) & -(\sigma+1)\left(\phi_{T_{i}}^{2 \sigma}-\phi^{2 \sigma}\left(\cdot+y_{T_{i}}^{(i-1)}-y^{(i-1)}\right)\right)
\end{array}\right), \\
& { }^{i \pi^{(i)}} \partial_{\pi} \tilde{W}_{T_{i}}\left(\pi^{(i-1)}\right) \\
& :=\dot{v}^{(i)}\left(\begin{array}{c}
\left(\cdot+y_{T_{i}}^{(i-1)}-y^{(i-1)}\right) e^{i \rho_{T_{i}}^{(i-1)}} \phi\left(\cdot+y_{T_{i}}^{(i-1)}-y^{(i-1)}\right) \\
\left(\cdot+y_{T_{i}}^{(i-1)}-y^{(i-1)}\right) e^{-i \rho_{T_{i}}^{(i-1)}} \phi\left(\cdot+y_{T_{i}}^{(i-1)}-y^{(i-1)}\right)
\end{array}\right) \\
& +\left(\dot{\gamma}^{(i)}+\dot{v}^{(i)} y^{(i-1)}\right)\left(\begin{array}{c}
-e^{i \rho_{T_{i}}^{(i-1)}} \phi\left(\cdot+y_{T_{i}}^{(i-1)}-y^{(i-1)}\right) \\
\left.e^{-i \rho_{T_{i}}^{(i-1)} \phi\left(\cdot+y_{T_{i}}^{(i-1)}-y^{(i-1)}\right)}\right)
\end{array}\right) \\
& +i \dot{\alpha}^{(i)}\left(\begin{array}{c}
e^{i \rho_{T_{i}}^{(i-1)}} \partial_{\alpha} \phi\left(\cdot+y_{T_{i}}^{(i-1)}-y^{(i-1)}\right) \\
e^{-i \rho_{T_{i}}^{(i-1)}} \partial_{\alpha} \phi\left(\cdot+y_{T_{i}}^{(i-1)}-y^{(i-1)}\right)
\end{array}\right) \\
& +i \dot{D}^{(i)}\left(\begin{array}{c}
-e^{i \rho_{T_{i}}^{(i-1)}} \partial_{x} \phi\left(\cdot+y_{T_{i}}^{(i-1)}-y^{(i-1)}\right) \\
-e^{-i \rho_{T_{i}}^{(i-1)}} \partial_{x} \phi\left(\cdot+y_{T_{i}}^{(i-1)}-y^{(i-1)}\right)
\end{array}\right), \\
& N_{T_{i}}\left(U, \pi^{(i-1)}\right):=\left(\begin{array}{l}
N_{1 T_{i}}\left(U, \pi^{(i-1)}\right) \\
N_{2 T_{i}}\left(U, \pi^{(i-1)}\right)
\end{array}\right)=\left(\begin{array}{c}
N_{1 T_{i}}\left(U, \pi^{(i-1)}\right) \\
-N_{1 T_{i}}\left(U, \pi^{(i-1)}\right)
\end{array}\right)
\end{aligned}
$$


and we have

$$
\begin{aligned}
& N_{1 T_{i}}\left(U, \pi^{(i-1)}\right) \\
& =-\left|U_{1}+e^{i \rho_{T_{i}}^{(i-1)}} \phi\left(\cdot+y_{T_{i}}^{(i-1)}-y^{(i-1)}\right)\right|^{2 \sigma}\left(U_{1}+e^{i \rho_{T_{i}}^{(i-1)}} \phi\left(\cdot+y_{T_{i}}^{(i-1)}-y^{(i-1)}\right)\right) \\
& +\phi\left(\cdot+y_{T_{i}}^{(i-1)}-y^{(i-1)}\right)^{2 \sigma+1} e^{i \rho_{T_{i}}^{(i-1)}}+(\sigma+1) \phi\left(\cdot+y_{T_{i}}^{(i-1)}-y^{(i-1)}\right)^{2 \sigma} U_{1} \\
& +\sigma \phi\left(\cdot+y_{T_{i}}^{(i-1)}-y^{(i-1)}\right)^{2 \sigma} e^{2 i \rho_{T_{i}}^{(i-1)}} U_{2} .
\end{aligned}
$$

As can be seen from (4.6), we need to define

$$
\dot{\tilde{\gamma}}^{(i)}=\dot{\gamma}^{(i)}+\dot{v}^{(i)} y^{(i-1)}
$$

and then, as usual, $\dot{\tilde{\pi}}^{(i)}=\left(\dot{\tilde{\gamma}}^{(i)}, \dot{v}^{(i)}, \dot{D}^{(i)}, \dot{\alpha}^{(i)}\right)$ and $\tilde{\pi}^{(i)}(0)=\left(0,0,0, \alpha_{0}\right)$. As above, we will sometimes write $\dot{\tilde{\pi}}^{(i)} \partial_{\pi} \tilde{W}_{T_{i}}\left(\pi^{(i-1)}\right)$ instead of $\dot{\pi}^{(i)} \partial_{\pi} \tilde{W}_{T_{i}}\left(\pi^{(i-1)}\right)$ to emphasize that we are working with $\tilde{\pi}^{(i)}$ rather than $\pi^{(i)}$ itself.

We shall analyze (4.5) on the interval $\left[0, T_{i}\right]$ and establish control over $\left\|U^{(i)}\right\|_{X_{*}\left(\left[0, T_{i}\right]\right)}$, upon defining $h$ suitably. More precisely, we shall establish the following

Proposition 4.4. There exists a canonically determined value

$$
h\left(R_{0}, Z^{(i-1)}, \pi^{(i-1)}, \pi^{(i-2)}\right) \in \mathbb{R}
$$

such that with initial data (4.4) and the assumptions of Theorem 4.2, we have for some universal constant $C_{0}$ independent of $A, C$

$$
\left\|\left(\pi^{(i)}, U^{(i)}\right)\right\|_{X_{*}\left(\left[0, T_{i}\right]\right)} \lesssim C^{2}[(A+1) \delta]^{2}+C^{2 \sigma+1} \delta^{2 \sigma+1}+C_{0} \delta .
$$

This proposition allows us to retrieve the a priori bound on $\left(\pi^{(i)}, U^{(i)}\right)$, respectively, $\left(\pi^{(i)}, Z^{(i)}\right)$; in order to establish Theorem 4.2, we still need to retrieve control over

$$
T_{i} \sup _{0 \leq t \leq T_{i}}\left|\pi^{(i)}(t)-\pi^{(i-1)}(t)\right|
$$

This follows from the next proposition.

Proposition 4.5. Assume $h$ is chosen as in the preceding theorem and, moreover, $A, C>1$ as above. Introduce the norm

$$
\begin{aligned}
\|(\pi, Z)\|_{Y^{(i)}([0, T])}:= & \sup _{t \in[0, T]}\langle t\rangle^{1+\frac{\epsilon}{2}}|\dot{\pi}(t)| \\
& +\sup _{t \in[0, T]}\left[\langle t\rangle^{-1}|| Z(t) \|_{L_{x}^{2}}\right. \\
& +\left\|\left\langle x-y^{(i-1)}(t)\right\rangle^{-\theta} Z(t)\right\|_{\left.L_{x}^{\infty}\right]}
\end{aligned}
$$

where $\theta=\frac{1}{2}+\epsilon$. Then we have the inequality 9

$$
\begin{aligned}
& \left\|\left(\tilde{\pi}^{(i)}-\tilde{\pi}^{(i-1)}, Z^{(i)}-Z^{(i-1)}\right)\right\|_{Y^{(i)}\left(\left[0, T_{i}\right]\right)} \\
& \lesssim\left[A^{2} C \delta+A^{2}(C \delta)^{2 \sigma}\right]\left\|\left(\tilde{\pi}^{(i-1)}-\tilde{\pi}^{(i-2)}, Z^{(i-1)}-Z^{(i-2)}\right)\right\|_{Y^{(i-1)}\left(\left[0, T_{i-1}\right]\right)} \\
& +\left[1+(A+1)^{2}(C \delta)^{2}+(C \delta)^{2 \sigma}\right] T_{i}^{-1} .
\end{aligned}
$$

${ }^{9}$ The statement here is far from optimal, but it is all that is needed to close the iteration. 
Let us now assume that these two propositions hold. Then we can finish the proof of Theorem 4.2. Observe that if we iterate the inequality of the second proposition, we get

$$
\begin{aligned}
& \sup _{t \in\left[0, T_{i}\right]}\langle t\rangle^{1+\frac{\epsilon}{2}}\left|\dot{\tilde{\pi}}^{(i)}-\dot{\tilde{\pi}}^{(i-1)}\right|(t) \lesssim\left[1+(A+1)^{2}(C \delta)^{2}+(C \delta)^{2 \sigma}\right] T_{i}^{-1} \\
& +A^{2}[C \delta \\
& \left.+(C \delta)^{2 \sigma}\right]\left[1+(A+1)^{2}(C \delta)^{2}+(C \delta)^{2 \sigma}\right] T_{i-1}^{-1} \\
& +\ldots+\left(A^{2}\left[C \delta+(C \delta)^{2 \sigma}\right]\right)^{i}\left[1+(A+1)^{2}(C \delta)^{2}+(C \delta)^{2 \sigma}\right] T_{0}^{-1} .
\end{aligned}
$$

We conclude that if we choose $A, C_{0}$ large enough and then $\delta$ small enough, we can bound

$$
\left\|\left(\pi^{(i)}, U^{(i)}\right)\right\|_{X_{*}\left(\left[0, T_{i}\right]\right)}<C \delta, T_{i} \sup _{t \in\left[0, T_{i}\right]}\langle t\rangle^{1+\frac{\epsilon}{2}}\left|\dot{\tilde{\pi}}^{(i)}-\dot{\tilde{\pi}}^{(i-1)}\right|(t)<A,
$$

which suffices to close the iteration.

Thus we are left with proving the two propositions. This will be established by means of a sequence of estimates.

Proof. Proof of Proposition 4.4 We shall estimate the various parts constituting the norm $\left\|\left(\pi^{(i)}, U^{(i)}\right)\right\|_{X_{*}\left(\left[0, T_{i}\right]\right)}$. We commence with the parts concerning $U^{(i)}$. We shall decompose $U^{(i)}$ into its dispersive, root, and hyperbolic parts with respect to the operator $\mathcal{H}\left(\alpha_{T_{i}}^{(i-1)}\right)$. Thus we split

$$
U^{(i)}=U_{d i s}^{(i)}+U_{\text {root }}^{(i)}+U_{\text {hyp }}^{(i)}
$$

and write

$$
U_{\text {root }}^{(i)}(t)=\sum_{j=1}^{4} \tilde{a}_{j}^{(i)}(t) \tilde{\eta}_{j}\left(\alpha_{T_{j}}^{(i-1)}\right), \quad U_{\text {hyp }}^{(i)}=b^{(i)+}(t) f^{+}\left(\alpha_{T_{i}}^{(i-1)}\right)+b^{(i)-}\left(\alpha_{T_{i}}^{(i-1)}\right) .
$$

We mean here that $U_{\text {root }}^{(i)}(t)=P_{0}\left(\alpha_{T_{j}}^{(i-1)}\right) U^{(i)}$ and $U_{\text {hyp }}^{(i)}=P_{I m}^{ \pm}\left(\alpha_{T_{j}}^{(i-1)}\right) U^{(i)}$, where $P_{I m}^{ \pm}\left(\alpha_{T_{j}}^{(i-1)}\right)$ projects onto the eigenspace corresponding to eigenvalue $\pm i \gamma\left(\alpha_{T_{j}}^{(i-1)}\right)$, respectively. The orthogonality condition $\left\langle Z^{(i)}, \xi_{\ell}\left(\pi^{(i-1)}\right)\right\rangle=0, \ell=1,2,3,4$, which is equivalent to $\left\langle U^{(i)}, \tilde{\xi}_{\ell}\left(\pi^{(i-1)}\right)\right\rangle=0$, implies an equation of the form

$$
\left\langle U_{d i s}^{(i)}, \tilde{\xi}_{\ell}\left(\pi^{(i-1)}\right)\right\rangle+\left\langle U_{h y p}^{(i)}, \tilde{\xi}_{\ell}\left(\pi^{(i-1)}\right)\right\rangle+\sum_{j=1}^{4} \tilde{a}_{j}^{(i)}(t)\left\langle\tilde{\eta}_{j}\left(\alpha_{T_{j}}^{(i-1)}\right), \tilde{\xi}_{\ell}\left(\pi^{(i-1)}\right)\right\rangle=0 .
$$

Our a priori assumptions on $\pi^{(i-1)}$ imply, upon choosing $\delta$ small enough, that the $4 \times 4$ matrix with entries $\left\langle\tilde{\eta}_{j}\left(\alpha_{T_{j}}^{(i-1)}\right), \tilde{\xi}_{\ell}\left(\pi^{(i-1)}\right)\right\rangle$ is nonsingular. In particular, we can deduce formulae $a_{j}=a_{j}\left(U_{\text {root }}^{(i)}, U_{\text {hyp }}^{(i)}, \pi^{(i-1)}\right)$. We feed this information back into (4.5). Then we specialize this equation to the dispersive and hyperbolic parts: for the dispersive part, we get

$$
\begin{aligned}
& i \dot{U^{(i)}}{ }_{d i s}(t)+\left(\begin{array}{cc}
\partial_{x}^{2}+(\sigma+1) \phi_{T_{i}}^{2 \sigma}-\left(\alpha_{T_{i}}^{(i-1)}\right)^{2} & \sigma \phi_{T_{i}}^{2 \sigma} \\
-\sigma \phi_{T_{i}}^{2 \sigma} & -\partial_{x}^{2}-(\sigma+1) \phi_{T_{i}}^{2 \sigma}+\left(\alpha_{T_{i}}^{(i-1)}\right)^{2}
\end{array}\right) U_{d i s}^{(i)} \\
& =P_{s}\left(\alpha_{T_{i}}^{(i-1)}\right)\left\{-i \dot{\tilde{\pi}}^{(i)} \partial_{\pi} \tilde{W}_{T_{i}}\left(\pi^{(i-1)}\right)+V^{(i-1)}\left[U_{\text {dis }}^{(i)}+U_{\text {hyp }}^{(i)}+U_{\text {root }}^{(i)}\left(U_{\text {dis }}^{(i)}, U_{\text {hyp }}^{(i)}\right)\right]\right. \\
& \left.+N_{T_{i}}\left(M_{T_{i-1}}\left(\pi^{(i-1)}\right) \mathcal{G}_{T_{i-1}}\left(\pi^{(i-1)}\right) \mathcal{G}_{T_{i-2}}\left(\pi^{(i-2)}\right)^{-1} M_{T_{i-2}}\left(\pi^{(i-2)}\right)^{-1} U^{(i-1)}, \pi^{(i-1)}\right)\right\} .
\end{aligned}
$$


For the hyperbolic part, we need to get a condition on $b^{(i)}{ }^{ \pm}$: denoting the hyperbolic projection of the right-hand side of (4.5) as

$$
\left(\begin{array}{c}
g^{+}\left(U^{(i)}, U^{(i-1)}, \pi^{(i)}, \pi^{(i-1)}, \pi^{(i-2)}\right) \\
g^{-}\left(U^{(i)}, U^{(i-1)}, \pi^{(i)}, \pi^{(i-1)}, \pi^{(i-2)}\right)
\end{array}\right)
$$

we can formulate the system

$$
\frac{d}{d t}\left(\begin{array}{l}
b^{(i)+} \\
b^{(i)-}
\end{array}\right)+\left(\begin{array}{cc}
-\gamma\left(\alpha_{T_{i}}^{(i-1)}\right) & 0 \\
0 & \gamma\left(\alpha_{T_{i}}^{(i-1)}\right)
\end{array}\right)\left(\begin{array}{l}
b^{(i)+} \\
b^{(i)-}
\end{array}\right)=\left(\begin{array}{l}
g^{+} \\
g^{-}
\end{array}\right)
$$

In order to control the growth of $b^{(i)+}$, we use the following lemma already used in 44:

Lemma 4.6. Consider the two-dimensional $O D E$

$$
\dot{x}(t)-A_{0} x(t)=f(t), \quad x(0)=\left(\begin{array}{c}
x_{1}(0) \\
x_{2}(0)
\end{array}\right)
$$

where $f=\left(\begin{array}{l}f_{1} \\ f_{2}\end{array}\right) \in L^{\infty}\left([0, \infty), \mathbb{C}^{2}\right)$ and $A_{0}=\left(\begin{array}{cc}\gamma & 0 \\ 0 & -\gamma\end{array}\right)$ where $\gamma>0$. Then $x(t)=\left(\begin{array}{l}x_{1}(t) \\ x_{2}(t)\end{array}\right)$ remains bounded for all times iff

$$
0=x_{1}(0)+\int_{0}^{\infty} e^{-\gamma t} f_{1}(t) d t
$$

Moreover, in that case

$$
x_{1}(t)=-\int_{t}^{\infty} e^{-(s-t) \gamma} f_{1}(s) d s, \quad x_{2}(t)=e^{-t \gamma} x_{2}(0)+\int_{0}^{t} e^{-(t-s) \gamma} f_{2}(s) d s
$$

for all $t \geq 0$.

Proof. Clearly, $x_{1}(t)=e^{t \gamma} x_{1}(0)+\int_{0}^{t} e^{(t-s) \gamma} f_{1}(s) d s$ and $x_{2}(t)=e^{-t \gamma} x_{2}(0)+$ $\int_{0}^{t} e^{-(t-s) \gamma} f_{2}(s) d s$. If

$$
\lim _{t \rightarrow \infty} e^{-t \gamma} x_{1}(t)=0
$$

then $0=x_{1}(0)+\int_{0}^{\infty} e^{-s \gamma} f_{1}(s) d s$, which is (4.9). Conversely, if this holds, then $x_{1}(t)=-e^{t \gamma} \int_{t}^{\infty} e^{-s \gamma} f_{1}(s) d s$, and the lemma is proved.

Of course we are working on a finite time interval, but we use this lemma to motivate our choice of $b^{(i)+}(0)$, namely

$$
b^{(i)+}(0)=-\int_{0}^{T_{i}} e^{-\gamma\left(\alpha_{T_{i}}^{(i-1)}\right) t} g^{+}\left(U^{(i)}, U^{(i-1)}, \pi^{(i)}, \pi^{(i-1)}, \pi^{(i-2)}\right)(t) d t .
$$


We now claim that there is a unique choice of $h^{(i)}$ for which we have

$$
\begin{aligned}
P_{I m}^{+}\left(\alpha_{T_{i}}^{(i-1)}\right)\left\{\mathcal { G } _ { T _ { i } } ( \pi ^ { ( i - 1 ) } ( 0 ) ) \left[\left(\frac{R_{0}}{R_{0}}\right)+h^{(i)} f^{+}\left(\alpha_{T_{i}}^{(i-1)}\right)\right.\right. \\
\left.\left.+\sum_{j=1}^{4} a_{j}^{(i)}\left(h^{(i)}, \alpha_{T_{i}}^{(i-1)}\right) \tilde{\eta}_{j}\left(\alpha_{T_{i}}^{(i-1)}\right)\right]\right\}=b^{(i)+}(0) .
\end{aligned}
$$

This follows from the fact that due to our assumption 10 , we have

$$
P_{I m}\left(\alpha_{T_{i}}^{(i-1)}\right)\left[\left(\mathcal{G}_{T_{i}}\left(\pi^{(i-1)}(0)\right)-I\right)\left(\frac{R_{0}}{R_{0}}\right)\right]=O(\delta),
$$

$$
P_{I m}\left(\alpha_{T_{i}}^{(i-1)}\right)\left[\left(\mathcal{G}_{T_{i}}\left(\pi^{(i-1)}(0)\right)-I\right) \sum_{j=1}^{4} a_{j}^{(i)}\left(h^{(i)}, \alpha_{T_{i}}^{(i-1)}\right) \tilde{\eta}_{j}\left(\alpha_{T_{i}}^{(i-1)}\right)\right]=O\left(\delta h^{(i)}\right) .
$$

Indeed, the dependence of $h^{(i)}$ on $b^{(i)+}(0)$ is linear. One also sees from the $\mathcal{J}$ invariance of $f^{+}\left(\alpha_{T_{i}}^{(i-1)}\right)$ as well as the root space representatives and the $\mathcal{J}$ invariance of the equations that $b^{(i)+}(t)$ is always real-valued, whence so is $h^{(i)}$. With this $h^{(i)}$, we can then define

$$
\begin{aligned}
U_{d i s}^{(i)}(0):=P_{s}\left(\alpha_{T_{i}}^{(i-1)}\right)\left\{\mathcal{G}_{T_{i}}\left(\pi^{(i-1)}(0)\right)[\right. & \left(\frac{R_{0}}{R_{0}}\right)+h^{(i)} f^{+}\left(\alpha_{T_{i}}^{(i-1)}\right) \\
& \left.\left.+\sum_{j=1}^{4} a_{j}^{(i)}\left(h^{(i)}, \alpha_{T_{i}}^{(i-1)}\right) \tilde{\eta}_{j}\left(\alpha_{T_{i}}^{(i-1)}\right)\right]\right\} .
\end{aligned}
$$

Similarly, we can uniquely specify $b^{(i)-}(0)$. Of course, solving for $U_{d i s}^{(i)}, U_{h y p}^{(i)}$ is complicated by the fact that the unknowns are on both sides of the equations (and indeed also implicitly determine the initial data). Thus we need to run a contraction argument to solve for them. Specifically, denoting

$$
h^{(i)}=h^{(i)}\left(U_{\text {dis }}^{(i)}, U_{\text {hyp }}^{(i)}, U^{(i-1)}, \pi^{(i-1)}, \pi^{(i-2)}\right)
$$

in the sense just established, we introduce a map $F_{T_{i}}\left(U^{(i-1)}, \pi^{(i-1)}, \pi^{(i-2)}\right)$, which sends a given pair $\left(\pi^{*}, U^{*}\right)$ satisfying the orthogonality relations

$$
\left\langle U^{*}, \tilde{\xi}_{\ell}\left(\pi^{(i-1)}\right)\right\rangle=0, \quad \ell=1,2,3,4,
$$

\footnotetext{
${ }^{10}$ Provided we choose $\delta>0$ small enough.
} 
into another one (satisfying the same orthogonality relations) $(\pi, U)$ as follows:

$$
\begin{aligned}
& i \dot{U}_{d i s}(t)+\left(\begin{array}{cc}
\partial_{x}^{2}+(\sigma+1) \phi_{T_{i}}^{2 \sigma}-\left(\alpha_{T_{i}}^{(i-1)}\right)^{2} & \sigma \phi_{T_{i}}^{2 \sigma} \\
-\sigma \phi_{T_{i}}^{2 \sigma} & -\partial_{x}^{2}-(\sigma+1) \phi_{T_{i}}^{2 \sigma}+\left(\alpha_{T_{i}}^{(i-1)}\right)^{2}
\end{array}\right) U_{d i s} \\
& =P_{s}\left(\alpha_{T_{i}}^{(i-1)}\right)\left[-i \dot{\tilde{\pi}}^{*} \partial_{\pi} \tilde{W}_{T_{i}}\left(\pi^{(i-1)}\right)+V^{(i-1)}\left(U_{d i s}^{*}+U_{h y p}^{*}+U_{\text {root }}^{*}\left(U_{d i s}^{*}, U_{h y p}^{*}\right)\right)\right. \\
& \left.+N_{T_{i}}\left(M_{T_{i-1}}\left(\pi^{(i-1)}\right) \mathcal{G}_{T_{i-1}}\left(\pi^{(i-1)}\right) \mathcal{G}_{T_{i-2}}\left(\pi^{(i-2)}\right)^{-1} M_{T_{i-2}}\left(\pi^{(i-2)}\right)^{-1} U^{(i-1)}, \pi^{(i-1)}\right)\right],
\end{aligned}
$$

$U_{d i s}(0)=$

$$
\begin{gathered}
P_{s}\left(\alpha_{T_{i}}^{(i-1)}\right)\left\{\mathcal { G } _ { T _ { i } } ( \pi ^ { ( i - 1 ) } ( 0 ) ) \left[\left(\frac{R_{0}}{R_{0}}\right)+h^{(i)}\left(U_{d i s}^{*}, U_{h y p}^{*}, U^{(i-1)}, \pi^{(i-1)}, \pi^{(i-2)}\right) f^{+}\left(\alpha_{T_{i}}^{(i-1)}\right)\right.\right. \\
\left.\left.+\sum_{j=1}^{4} a_{j}^{(i)}\left(h^{(i)}\left(U_{d i s}^{*}, U_{h y p}^{*}, U^{(i-1)}, \pi^{(i-1)}, \pi^{(i-2)}\right), \alpha_{T_{i}}^{(i-1)}\right) \tilde{\eta}_{j}\left(\alpha_{T_{i}}^{(i-1)}\right)\right]\right\},
\end{gathered}
$$

$$
\begin{aligned}
& \frac{d}{d t}\left(\begin{array}{l}
b^{+} \\
b^{-}
\end{array}\right)+\left(\begin{array}{cc}
-\gamma\left(\alpha_{T_{i}}^{(i-1)}\right) & 0 \\
0 & \gamma\left(\alpha_{T_{i}}^{(i-1)}\right)
\end{array}\right)\left(\begin{array}{l}
b^{+} \\
b^{-}
\end{array}\right) \\
& =\left(\begin{array}{l}
g^{+}\left(U^{*}, U^{(i-1)}, \pi^{*}, \pi^{(i-1)}, \pi^{(i-2)}\right) \\
g^{-}\left(U^{*}, U^{(i-1)}, \pi^{*}, \pi^{(i-1)}, \pi^{(i-2)}\right)
\end{array}\right),
\end{aligned}
$$

$$
\begin{aligned}
& b^{+}(0)= b^{+}\left(U^{*}, U^{(i-1)}, \pi^{*}, \pi^{(i-1)}, \pi^{(i-2)}\right), b^{-}(0)=b^{-}\left(U^{*}, U^{(i-1)}, \pi^{*}, \pi^{(i-1)}, \pi^{(i-2)}\right), \\
&\left\langle i \dot{\tilde{\pi}} \partial_{\pi} \tilde{W}_{T_{i}}\left(\pi^{(i-1)}\right), \tilde{\xi}_{\ell}\left(\pi^{(i-1)}\right)\right\rangle=-\left\langle U^{*}(t), i \dot{\tilde{\pi}}^{(i-1)} \tilde{\mathcal{S}}_{\ell}\left(.+y_{T}^{(i-1)}-y^{(i-1)}\right)(t)\right\rangle \\
&+\left\langleN _ { T _ { i } } \left( M_{T_{i-1}}\left(\pi^{(i-1)}\right) \mathcal{G}_{T_{i-1}}\left(\pi^{(i-1)}\right) \mathcal{G}_{T_{i-2}}\left(\pi^{(i-2)}\right)^{-1}\right.\right. \\
&\left.\left.\quad \times M_{T_{i-2}}\left(\pi^{(i-2)}\right)^{-1} U^{(i-1)}, \pi^{(i-1)}\right), \tilde{\xi}_{\ell}\left(\pi^{(i-1)}(t)\right)\right\rangle .
\end{aligned}
$$

Here $\tilde{\mathcal{S}}_{\ell}$ is from Lemma 3.4. Of course we have written $U_{\text {hyp }}=b^{+} f^{+}\left(\alpha_{T_{i}}^{(i-1)}\right)+$ $b^{-} f^{-}\left(\alpha_{T_{i}}^{(i-1)}\right)$, and this in addition to $U_{\text {dis }}$ uniquely determines the root part $U_{\text {root }}$, on account of the orthogonality relations $\left\langle U, \tilde{\xi}_{\ell}\left(\pi^{(i-1)}\right)\right\rangle=0$, which we assume to hold. Our task is to find a fixed point for the affine map

$$
F_{T_{i}}\left(U^{(i-1)}, \pi^{(i-1)}, \pi^{(i-2)}\right):\left(\pi^{*}, U^{*}\right) \mapsto(\pi, U) .
$$

In order to do this, we need to show that it is a contraction with respect to the norm $\|\cdot\|_{X_{*}\left(\left[0, T_{i}\right]\right)}$. This will then define the iterate $\left(\pi^{(i)}, U^{(i)}\right)$ and thereby (undoing the gauge) $\left(\pi^{(i)}, Z^{(i)}\right)$. Note that equations (4.12)-(4.15) can all be solved by integration in terms of the initial data and the right-hand sides.

We shall show here that $F_{T_{i}}\left(U^{(i-1)}, \pi^{(i-1)}, \pi^{(i-2)}\right)$ sends the ball

$$
\|(\pi, U)\|_{X_{*}\left(\left[0, T_{i}\right]\right)}<C_{0} C^{2}[(A+1) \delta]^{2}+C^{2 \sigma+1} \delta^{2 \sigma+1}+C_{0} \delta=: M\left(A, C, C_{0}, \delta\right)
$$

into itself, provided $\delta$ is small enough in relation to $A, C, C_{0}$ and provided the latter quantities are large enough (relative to some absolute constant, and with $C_{0}$ small enough in relation to $C$ ). The same estimates, when we consider a suitable difference equation, will establish the contraction property, as well as the inequality in Proposition 4.4 
(A): Estimating $\left\|U_{d i s}\right\|_{L_{t}^{\infty} L_{x}^{2}\left(\left[0, T_{i}\right]\right)}$ : for this we use the fact that

$$
\begin{aligned}
\left\|U_{d i s}\right\|_{L_{t}^{\infty} L_{x}^{2}\left(\left[0, T_{i}\right]\right)} \lesssim \sup _{t \in\left[0, T_{i}\right]}\left\|e^{i t \mathcal{H}\left(\alpha_{T_{i}}^{(i-1)}\right)} U_{d i s}(0)\right\|_{L_{x}^{2}} \\
+\| \text { right-hand side of (4.12) } \|_{L_{t}^{1} L_{x}^{2}\left(\left[0, T_{i}\right]\right)} .
\end{aligned}
$$

We commence by estimating the various terms on the right-hand side of (4.12): first, observe that

$P_{s}\left(\alpha_{T_{i}}^{(i-1)}\right)\left[i \dot{\tilde{\pi}}^{*} \partial_{\pi} \tilde{W}_{T_{i}}\left(\pi^{(i-1)}\right)\right]=P_{s}\left(\alpha_{T_{i}}^{(i-1)}\right)\left[i \dot{\tilde{\pi}}^{*} \partial_{\pi} \tilde{W}_{T_{i}}\left(\pi^{(i-1)}\right)-i \dot{\tilde{\pi}}^{*} \partial_{\pi} \tilde{W}_{T_{i}}\left(\pi_{T_{i}}^{(i-1)}\right)\right]$, where $\partial_{\pi} \tilde{W}_{T_{i}}\left(\pi_{T_{i}}^{(i-1)}\right)$ is the same as $\partial_{\pi} \tilde{W}_{T_{i}}\left(\pi^{(i-1)}\right)$ with the path $\pi^{(i-1)}$ replaced by the straight line path $\pi_{T_{i}}^{(i-1)}$; see Definition 3.1, Lemma 3.2, and Lemma 2.4. Therefore, by our assumptions on $\pi^{(i-1)}$, we get

$$
\begin{aligned}
\left\|P_{S}\left(\alpha_{T_{i}}^{(i-1)}\right)\left[i \dot{\pi}^{*} \partial_{\pi} \tilde{W}_{T_{i}}\left(\pi^{(i-1)}\right)\right]\right\|_{L_{t}^{1} L_{x}^{2}\left(\left[0, T_{i}\right]\right)} & \lesssim \delta C\left[\sup _{t \in\left[0, T_{i}\right]}\langle t\rangle^{2+\epsilon}\left|\dot{\tilde{\pi}}^{*}\right|\right] \int_{0}^{T_{i}}\langle t\rangle^{-2-\epsilon} d t \\
& \lesssim C \delta M\left(A, C, C_{0}, \delta\right) .
\end{aligned}
$$

We have used the following simple lemma.

Lemma 4.7. Under the assumptions of Proposition 4.4, we have

$$
\left|\partial_{\pi} \tilde{W}_{T_{i}}\left(\pi^{(i-1)}\right)-\partial_{\pi} \tilde{W}_{T_{i}}\left(\pi_{T_{i}}^{(i-1)}\right)\right|(t) \lesssim C_{\epsilon} \delta\langle t\rangle^{-\epsilon} .
$$

Proof. This follows from the definition of $\partial_{\pi} \tilde{W}_{T_{i}}\left(\pi^{(i-1)}\right)$ (with $y, y_{T}$ replaced by $\left.y^{(i-1)}, y_{T}^{(i-1)}\right)$, see the definition after (4.5), and the fact that

$$
\left|y^{(i-1)}(t)-y_{T}^{(i-1)}(t)\right| \lesssim C \delta\langle t\rangle^{-\epsilon},\left|\rho_{T}(t, x)\right| \leq C_{\varepsilon} \delta^{2}(1+|x|)\langle t\rangle^{-\varepsilon}
$$

as follows from Lemma 2.4

Next, using the definition of $V^{(i-1)}$ in (4.6), as well as the linear dependence of $U_{\text {root }}$ on $U_{d i s}, U_{\text {hyp }}$, we get

$$
\begin{aligned}
& \left\|V^{(i-1)}\left(U_{\text {dis }}^{*}+U_{\text {hyp }}^{*}+U_{\text {root }}^{*}\left(U_{\text {dis }}^{*}, U_{\text {hyp }}^{*}\right)\right)\right\|_{L_{t}^{1} L_{x}^{2}\left(\left[0, T_{i}\right]\right)} \\
& \lesssim\left\|V^{(i-1)}\langle x\rangle^{\theta}\right\|_{L_{t}^{\infty} L_{x}^{2}\left(\left[0, T_{i}\right]\right)}\left[\left\|\langle x\rangle^{-\theta} U_{d i s}^{*}\right\|_{L_{t}^{1} L_{x}^{\infty}\left(\left[0, T_{i}\right]\right)}+\left\|\langle x\rangle^{-\theta} U_{h y p}^{*}\right\|_{L_{t}^{1} L_{x}^{\infty}\left(\left[0, T_{i}\right]\right)}\right. \\
& \left.+\left\|\langle x\rangle^{-\theta} U_{\text {root }}^{*}\right\|_{L_{t}^{1} L_{x}^{\infty}\left(\left[0, T_{i}\right]\right)}\right] \\
& \lesssim C \delta\left\|\langle t\rangle^{1+\epsilon}\langle x\rangle^{-\theta} U^{*}\right\|_{L_{t}^{\infty} L_{x}^{\infty}} \int_{0}^{T_{i}}\langle t\rangle^{-1-\epsilon} d t \lesssim C \delta M\left(A, C, C_{0}, \delta\right) .
\end{aligned}
$$

We proceed to the last and most complicated term of the nonlinearity. To simplify notation, denote

$$
\tilde{U}^{(i-1)}:=M_{T_{i-1}}\left(\pi^{(i-1)}\right) \mathcal{G}_{T_{i-1}}\left(\pi^{(i-1)}\right) \mathcal{G}_{T_{i-2}}\left(\pi^{(i-2)}\right)^{-1} M_{T_{i-2}}\left(\pi^{(i-2)}\right)^{-1} U^{(i-1)} .
$$

Thus we need to estimate $\left\|N_{T_{i}}\left(\tilde{U}^{(i-1)}, \pi^{(i-1)}\right)\right\|_{L_{t}^{1} L_{x}^{2}\left(\left[0, T_{i}\right]\right.}$. Using Lemma 2.7, we get

$$
\begin{aligned}
\left|N_{T_{i}}\left(\tilde{U}^{(i-1)}, \pi^{(i-1)}\right)\right|(t, x) & \lesssim\left|\tilde{U}^{(i-1)}(x, t)\right|^{2 \sigma+1} \\
& +\left|\tilde{U}^{(i-1)}(x, t)\right|^{2} \phi^{2 \sigma-1}\left(x+\left(y_{T_{i}}^{(i-1)}-y^{(i-1)}\right)(t)\right) .
\end{aligned}
$$


We first estimate the contribution of the local term. We have

$$
\begin{aligned}
& \left\|\tilde{U}^{(i-1)}(x, t)^{2} \phi^{2 \sigma-1}\left(x+\left(y_{T_{i}}^{(i-1)}-y^{(i-1)}\right)(t)\right)\right\|_{L_{t}^{1} L_{x}^{2}\left(\left[0, T_{i}\right]\right)} \\
& \lesssim\left\|\langle x\rangle^{-2 \theta} \tilde{U}^{(i-1)}(x, t)^{2}\right\|_{L_{t}^{1} L_{x}^{\infty}}\left\|\langle x\rangle^{2 \theta} \phi^{2 \sigma-1}\left(x+\left(y_{T_{i}}^{(i-1)}-y^{(i-1)}\right)(t)\right)\right\|_{L_{t}^{\infty} L_{x}^{2}} \\
& \lesssim(A+1) C \delta\left\|\langle t\rangle^{1+\epsilon}\langle x\rangle^{-\theta} U^{(i-1)}\right\|_{L_{t}^{\infty} L_{x}^{\infty}}\left[\int_{0}^{T_{i}}\langle t\rangle^{-1-\epsilon} t^{-\frac{1}{2}} d t\right] \lesssim(A+1) C^{2} \delta^{2} .
\end{aligned}
$$

We have crudely bounded for $t \in\left[0, T_{i}\right]$

$$
\sup _{x \in \mathbb{R}}\langle x\rangle^{-\theta}\left|\tilde{U}^{(i-1)}\right|(t, x) \lesssim(A+1) \sup _{x \in \mathbb{R}}\langle x\rangle^{-\theta}\left|U^{(i-1)}\right|(t, x) .
$$

Finally, we need to estimate $\left\|\left(\tilde{U}^{(i-1)}\right)^{2 \sigma+1}\right\|_{L_{t}^{1} L_{x}^{2}\left(\left[0, T_{i}\right]\right)}$. This is straightforward. We have

$$
\left\|\left(\tilde{U}^{(i-1)}\right)^{2 \sigma+1}\right\|_{L_{t}^{1} L_{x}^{2}\left(\left[0, T_{i}\right]\right)} \lesssim\left\|U^{(i-1)}\right\|_{L_{t}^{1} L_{x}^{\infty}}^{2 \sigma}\left\|U^{(i-1)}\right\|_{L_{t}^{\infty} L_{x}^{2}} .
$$

Note that the translations and phase functions distinguishing $\tilde{U}^{(i-1)}$ from $U^{(i-1)}$ are irrelevant here. One bounds the preceding by

$$
\lesssim C \delta \int_{1}^{T_{i}} t^{-\sigma}\left\|t^{\frac{1}{2}} U^{(i-1)}(t)\right\|_{L_{x}^{\infty}}^{2 \sigma} d t+C \delta \int_{0}^{1}\left[\left\|\partial_{x} U^{(i-1)}(t)\right\|_{L_{x}^{2}}^{2 \sigma}+\left\|U^{(i-1)}(t)\right\|_{L_{x}^{2}}^{2 \sigma}\right] d t,
$$

where we have used Sobolev's inequality in the last step. The expression can be bounded by $\lesssim(C \delta)^{2 \sigma+1}$. To finish the estimation of $U_{d i s}$, we still need to handle the free contribution, i.e., $\sup _{t \in\left[0, T_{i}\right]}\left\|e^{i t \mathcal{H}\left(\alpha_{T_{i}}^{(i-1)}\right)} U_{d i s}(0)\right\|_{L_{x}^{2}}$. For this we need to carefully keep track of the definition of $U_{d i s}(0)$, which was

$$
\begin{aligned}
& U_{\text {dis }}(0)= \\
& P_{s}\left(\alpha_{T_{i}}^{(i-1)}\right)\left\{\mathcal { G } _ { T _ { i } } ( \pi ^ { ( i - 1 ) } ( 0 ) ) \left[\left(\frac{R_{0}}{R_{0}}\right)+h^{(i)}\left(U_{d i s}^{*}, U_{h y p}^{*}, U^{(i-1)}, \pi^{(i-1)}, \pi^{(i-2)}\right) f^{+}\left(\alpha_{T_{i}}^{(i-1)}\right)\right.\right. \\
& \left.\left.\quad+\sum_{j=1}^{4} a_{j}^{(i)}\left(h^{(i)}\left(U_{d i s}^{*}, U_{h y p}^{*}, U^{(i-1)}, \pi^{(i-1)}, \pi^{(i-2)}\right), \alpha_{T_{i}}^{(i-1)}\right) \tilde{\eta}_{j}\left(\alpha_{T_{i}}^{(i-1)}\right)\right]\right\} .
\end{aligned}
$$

Recall that $h^{(i)}\left(U_{d i s}^{*}, U_{h y p}^{*}, U^{(i-1)}, \pi^{(i-1)}, \pi^{(i-2)}\right)$ depended linearly on

$$
b^{(i)+}\left(U_{\text {dis }}^{*}, U_{\text {hyp }}^{*}, U^{(i-1)}, \pi^{(i-1)}, \pi^{(i-2)}\right),
$$

which in turn was given by the expression

$$
-\int_{0}^{T_{i}} e^{-\gamma\left(\alpha_{T_{i}}^{(i-1)}\right) t} g^{+}\left(U^{*}, U^{(i-1)}, \pi^{*}, \pi^{(i-1)}, \pi^{(i-2)}\right)(t) d t,
$$

where $g^{+}(\ldots)$ is as in (4.14). But this part is estimated exactly as above (indeed, we have an extra exponentially decaying weight), and one winds up with the estimate

$$
\begin{aligned}
& \left|\int_{0}^{T_{i}} e^{-\gamma\left(\alpha_{T_{i}}^{(i-1)}\right) t} g^{+}\left(U^{*}, U^{(i-1)}, \pi^{*}, \pi^{(i-1)}, \pi^{(i-2)}\right)(t) d t\right| \\
& \lesssim(A+1)(C \delta)^{2}+(C \delta)^{5}+C \delta M\left(A, C, C_{0}, \delta\right) .
\end{aligned}
$$


The fifth power here comes from $2 \sigma+1>5$. Keeping in mind the linear dependence of the $a_{j}^{(i)}$ on $h^{(i)}$ and choosing $C_{0}$ such that

$$
\left\|P_{s}\left(\alpha_{T_{i}}^{(i-1)}\right) \mathcal{G}_{T_{i}}\left(\pi^{(i-1)}(0)\right)\left(\frac{R_{0}}{R_{0}}\right)\right\|_{L_{x}^{2}}<\frac{C_{0}}{\Lambda} \delta,
$$

we get the estimate (for large $\Lambda$ )

$$
\left\|U_{d i s}(0)\right\|_{L_{x}^{2}}<\frac{C_{0}}{\Lambda} \delta+C_{1}\left[(A+1)(C \delta)^{2}+(C \delta)^{5}+C \delta M\left(A, C, C_{0}, \delta\right)\right]
$$

for suitable $C_{1}$. Using the approximate unitarity of the evolution $e^{i t \mathcal{H}\left(\alpha_{T_{i}}^{(i-1)}\right)}$ acting on $P_{s}\left(\alpha_{T_{i}}^{(i-1)}\right)\left(L^{2}\right)$, we finally obtain

$$
\left\|U_{d i s}\right\|_{L_{t}^{\infty} L_{x}^{2}\left(\left[0, T_{i}\right]\right)} \leq \frac{C_{0}}{\tilde{\Lambda}} \delta+C_{2}\left[(A+1)(C \delta)^{2}+(C \delta)^{5}+C \delta M\left(A, C, C_{0}, \delta\right)\right]
$$

for suitable $C_{2}$ (independent of all other constants) and large $\tilde{\Lambda}$, provided $\Lambda$ was chosen large enough.

(B): Estimating $\left\|U_{\text {root }}\right\|_{L_{t}^{\infty} L_{x}^{2}\left(\left[0, T_{i}\right]\right)}+\left\|U_{h y p}\right\|_{L_{t}^{\infty} L_{x}^{2}\left(\left[0, T_{i}\right]\right)}$. To complete the es-

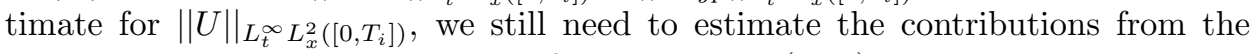
hyperbolic and root parts. For the former, we use (4.14), as well as the condition for $b^{+}(0)$ and Lemma 4.6, which results in

$$
\begin{gathered}
b^{+}(t)=-\int_{t}^{T_{i}} e^{-\gamma\left(\alpha_{T_{i}}^{(i-1)}\right)(s-t)} g^{+}\left(U^{*}, U^{(i-1)}, \pi^{*}, \pi^{(i-1)}, \pi^{(i-2)}\right)(t) d t \\
b^{-}(t)=e^{-t \gamma\left(\alpha_{T_{i}}^{(i-1)}\right)} b^{-}(0)+\int_{0}^{t} e^{\gamma\left(\alpha_{T_{i}}^{(i-1)}\right)(s-t)} g^{-}\left(U^{*}, U^{(i-1)}, \pi^{*}, \pi^{(i-1)}, \pi^{(i-2)}\right)(t) d t
\end{gathered}
$$

for all $t \in\left[0, T_{i}\right]$. One can then bound this by the same kind of expression as $\left\|U_{\text {dis }}\right\|_{L_{t}^{\infty} L_{x}^{2}\left(\left[0, T_{i}\right]\right)}$. Finally, the fact that $U_{\text {root }}$ depends linearly on $U_{\text {dis }}, U_{\text {hyp }}$ implies

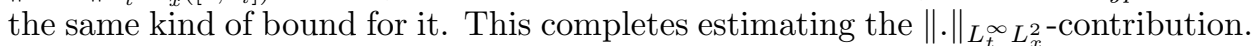

(C): The contribution of $\left\|\partial_{x} U\right\|_{L_{t}^{\infty} L_{x}^{2}}$. Using Corollary 8.3, as well as the Duhamel parametrix, we see that we need to estimate

$$
\| \partial_{x}\left[\text { right-hand side of (4.12) without the } P_{s}\right] \|_{L_{t}^{1} L_{x}^{2}\left(\left[0, T_{i}\right]\right)} \text {. }
$$

However, this follows from almost identical estimates. One simply substitutes $\left\|\langle t\rangle^{1+\epsilon}\langle x\rangle^{-\frac{1}{2}-2 \epsilon} \partial_{x} U\right\|_{L_{t}^{\infty} L_{x}^{q}}$ where before we used $\left\|\langle t\rangle^{1+\epsilon}\langle x\rangle^{-\theta} U\right\|_{L_{t}^{\infty} L_{x}^{\infty}\left[0, T_{i}\right]}$.

(D): The contribution of $\sup _{0 \leq \theta \leq 1}\langle t\rangle^{-\theta}\left\|\langle x\rangle^{\theta} U\right\|_{L_{x}^{2}}$. This is treated just like the preceding cases, using the Duhamel formula in addition to Lemma 6.12 (more precisely, an interpolate of this and the approximate $L^{2}$ conservation). The details are very similar to previous calculations, and we skip them. 
(E): Estimating the weighted norm $\left\|\langle t\rangle^{1+\epsilon}\langle x\rangle^{-\theta} U_{d i s}\right\|_{L_{t}^{\infty} L_{x}^{\infty}\left(\left[0, T_{i}\right]\right)}, \theta=\frac{1}{2}+\epsilon$. Using Duhamel's formula as well as Lemma 8.2 we see that we have11

$$
\begin{aligned}
& \left\|\langle x\rangle^{-\theta} U_{\text {dis }}(t)\right\|_{L_{x}^{\infty}} \lesssim\left\|\langle x\rangle^{-\theta} e^{i t \mathcal{H}\left(\alpha_{T_{i}}^{(i-1)}\right)} U_{\text {dis }}(0)\right\|_{L_{x}^{\infty}} \\
& +\int_{0}^{t-1}\langle t-s\rangle^{-1-\epsilon} \|\langle x\rangle^{\theta} P_{s}\left(\alpha_{T_{i}}^{(i-1)}\right)\left[i \dot{\pi}^{*} \partial_{\pi} \tilde{W}_{T_{i}}\left(\pi^{(i-1)}\right)\right. \\
& +V^{(i-1)}\left(U_{\text {dis }}^{*}+U_{\text {hyp }}^{*}+U_{\text {root }}^{*}\left(U_{\text {dis }}^{*}, U_{\text {hyp }}^{*}\right)\right) \\
& +N_{T_{i}}\left(M_{T_{i-1}}\left(\pi^{(i-1)}\right) \mathcal{G}_{T_{i-1}}\left(\pi^{(i-1)}\right) \mathcal{G}_{T_{i-2}}\left(\pi^{(i-2)}\right)^{-1}\right. \\
& \left.\left.\times M_{T_{i-2}}\left(\pi^{(i-2)}\right)^{-1} U^{(i-1)}, \pi^{(i-1)}\right)\right](s) \|_{L_{x}^{1}} d s \\
& +\int_{t-1}^{t}(t-s)^{-\frac{1}{2}} \| P_{s}\left(\alpha_{T_{i}}^{(i-1)}\right)\left[i \dot{\tilde{\pi}}^{*} \partial_{\pi} \tilde{W}_{T_{i}}\left(\pi^{(i-1)}\right)\right. \\
& +V^{(i-1)}\left(U_{\text {dis }}^{*}+U_{\text {hyp }}^{*}+U_{\text {root }}^{*}\left(U_{\text {dis }}^{*}, U_{\text {hyp }}^{*}\right)\right) \\
& +N_{T_{i}}\left(M_{T_{i-1}}\left(\pi^{(i-1)}\right) \mathcal{G}_{T_{i-1}}\left(\pi^{(i-1)}\right) \mathcal{G}_{T_{i-2}}\left(\pi^{(i-2)}\right)^{-1}\right. \\
& \left.\left.\times M_{T_{i-2}}\left(\pi^{(i-2)}\right)^{-1} U^{(i-1)}, \pi^{(i-1)}\right)\right](s) \|_{L_{x}^{1}} d s .
\end{aligned}
$$

For the last integral expression, we have rather crudely discarded the weight $\langle x\rangle^{-\theta}$, since this term turns out to be very small. We proceed as in case (A) by treating all the different terms. First, consider the first integral expression on the right. We estimate for $t \in\left[0, T_{i}\right]$

$$
\begin{aligned}
& \int_{0}^{t-1}\langle t-s\rangle^{-1-\epsilon}\left\|\langle x\rangle^{\theta} P_{s}\left(\alpha_{T_{i}}^{(i-1)}\right)\left[i \dot{\tilde{\pi}}^{*} \partial_{\pi} \tilde{W}_{T_{i}}\left(\pi^{(i-1)}\right)\right](s)\right\|_{L_{x}^{1}} d s \\
& =\int_{0}^{t-1}\langle t-s\rangle^{-1-\epsilon}\left\|\langle x\rangle^{\theta} P_{s}\left(\alpha_{T_{i}}^{(i-1)}\right)\left[i \dot{\tilde{\pi}}^{*} \partial_{\pi} \tilde{W}_{T_{i}}\left(\pi^{(i-1)}\right)-i \dot{\tilde{\pi}}^{*} \partial_{\pi} \tilde{W}_{T_{i}}\left(\pi_{T_{i}}^{(i-1)}\right)\right](s)\right\|_{L_{x}^{1}} d s \\
& \lesssim C \delta \int_{0}^{t-1}\langle t-s\rangle^{-1-\epsilon}\langle s\rangle^{-2-\epsilon} d s \lesssim C \delta M\left(A, C, C_{0}, \delta\right)\langle t\rangle^{-1-\epsilon} .
\end{aligned}
$$

This is acceptable in light of the definition of $\|\cdot\|_{X_{*}\left(\left[0, T_{i}\right]\right)}$. We have used the fact that we may safely move the weight $\langle x\rangle^{\theta}$ past $P_{s}\left(\alpha_{T_{i}}^{(i-1)}\right)$ and then discard it, due to the local nature of the expression. Next, we have

$$
\begin{aligned}
& \int_{0}^{t-1}\langle t-s\rangle^{-1-\epsilon}\left\|\langle x\rangle^{\theta} P_{s}\left(\alpha_{T_{i}}^{(i-1)}\right)\left[V^{(i-1)}\left(U_{\text {dis }}^{*}+U_{\text {hyp }}^{*}+U_{\text {root }}^{*}\left(U_{\text {dis }}^{*}, U_{\text {hyp }}^{*}\right)\right)\right](s)\right\|_{L_{x}^{1}} d s \\
& \lesssim \int_{0}^{t-1}\langle t-s\rangle^{-1-\epsilon}\left\|\langle x\rangle^{2 \theta} V^{(i-1)}(s)\right\|_{L_{x}^{1}}\left\|\langle x\rangle^{-\theta}\left(U_{\text {dis }}^{*}+U_{\text {hyp }}^{*}+U_{\text {root }}^{*}\left(U_{\text {dis }}^{*}, U_{\text {hyp }}^{*}\right)\right)(s)\right\|_{L_{x}^{\infty}} d s \\
& \lesssim C \delta M\left(A, C, C_{0}, \delta\right) \int_{0}^{t-1}\langle t-s\rangle^{-1-\epsilon}\langle s\rangle^{-1-\epsilon} d s \lesssim\langle t\rangle^{-1-\epsilon} C \delta M\left(A, C, C_{0}, \delta\right),
\end{aligned}
$$

as desired. Next, we consider

$$
\int_{0}^{t-1}\langle t-s\rangle^{-1-\epsilon}\left\|\langle x\rangle^{\theta} P_{s}\left(\alpha_{T_{i}}^{(i-1)}\right)\left[N_{T_{i}}\left(\tilde{U}^{(i-1)}, \pi^{(i-1)}\right)\right](s)\right\|_{L_{x}^{1}} d s .
$$

\footnotetext{
${ }^{11}$ The $s$ in $P_{s}\left(\alpha_{T_{i}}^{(i-1)}\right)$ stands for "stable" and has nothing to do with the integration variable $s$.
} 
As before, we move the weight past the operator $P_{s}\left(\alpha_{T_{i}}^{(i-1)}\right)$ and discard the latter, obtaining the expression

$$
\int_{0}^{t-1}\langle t-s\rangle^{-1-\epsilon}\left\|\langle x\rangle^{\theta}\left[N_{T_{i}}\left(\tilde{U}^{(i-1)}, \pi^{(i-1)}\right)\right](s)\right\|_{L_{x}^{1}} d s .
$$

We use Lemma 2.7, which implies

$$
\begin{aligned}
& \int_{0}^{t-1}\langle t-s\rangle^{-1-\epsilon}\left\|\langle x\rangle^{\theta} N_{T_{i}}\left(\tilde{U}^{(i-1)}, \pi^{(i-1)}\right)(s)\right\|_{L_{x}^{1}} d s \\
& \lesssim \int_{0}^{t-1}\langle t-s\rangle^{-1-\epsilon} \|\langle x\rangle^{\theta}\left[\left|\tilde{U}^{(i-1)}(x, s)\right|^{2 \sigma+1}\right. \\
& \left.+\left|\tilde{U}^{(i-1)}(x, s)\right|^{2} \phi^{2 \sigma-1}\left(x+\left(y_{T_{i}}^{(i-1)}-y^{(i-1)}\right)(s)\right)\right] \|_{L_{x}^{1}} d s .
\end{aligned}
$$

We treat each of the two summands separately. First, consider the local term. The weight is simply absorbed here; i.e., we can estimate

$$
\begin{aligned}
& \int_{0}^{t-1}\langle t-s\rangle^{-1-\epsilon}\left\|\langle x\rangle^{\theta}\left|\tilde{U}^{(i-1)}(x, s)\right|^{2} \phi^{2 \sigma-1}\left(x+\left(y_{T_{i}}^{(i-1)}-y^{(i-1)}\right)(s)\right)\right\|_{L_{x}^{1}} d s \\
& \lesssim \int_{0}^{t-1}\langle t-s\rangle^{-1-\epsilon}\left\|\langle x\rangle^{-\theta} \tilde{U}^{(i-1)}(x, s)\right\|_{L_{x}^{\infty}}\left\|\tilde{U}^{(i-1)}(x, s)\right\|_{L_{x}^{\infty}} \\
& \quad \times\left\|\langle x\rangle^{2 \theta} \phi^{2 \sigma-1}\left(x+\left(y_{T_{i}}^{(i-1)}-y^{(i-1)}\right)(s)\right)\right\|_{L_{x}^{1}} d s .
\end{aligned}
$$

Again exploiting (4.16) (actually, we only need to pay $A$ here), we can bound this integral by

$$
\lesssim A(C \delta)^{2} \int_{0}^{t-1}\langle t-s\rangle^{-1-\epsilon}\langle s\rangle^{-\frac{3}{2}} d s \lesssim A(C \delta)^{2}\langle t\rangle^{-1-\epsilon}
$$

Next, consider the nonlocal term. Here, we can no longer absorb the weights and therefore need to carefully keep track of the exact powers. For this purpose, we assume (as we may) that $0<\epsilon<\sigma-1-2 \theta$. Using Lemma 6.12, we get

$$
\begin{aligned}
& \int_{0}^{t-1}\langle t-s\rangle^{-1-\epsilon}\left\|\langle x\rangle^{\theta}\left|\tilde{U}^{(i-1)}(x, s)\right|^{2 \sigma+1}\right\|_{L_{x}^{1}} d s \\
& \lesssim \int_{0}^{t-1}\langle t-s\rangle^{-1-\epsilon}\left\|\langle x\rangle^{\theta} \tilde{U}^{(i-1)}(x, s)\right\|_{L_{x}^{2}}\left\|\tilde{U}^{(i-1)}(x, s)\right\|_{L_{x}^{2}}\left\|\tilde{U}^{(i-1)}(x, s)\right\|_{L_{x}^{\infty}}^{2 \sigma-1} d s \\
& \lesssim A(C \delta)^{2 \sigma+1} \int_{0}^{t-1}\langle t-s\rangle^{-1-\epsilon} s^{-\sigma+\frac{1}{2}+\theta} d s \lesssim A(C \delta)^{2 \sigma+1}\langle t\rangle^{-1-\epsilon} .
\end{aligned}
$$

We have crudely bounded

$$
\sup _{x \in \mathbb{R}}\left|\langle x\rangle^{\theta} \tilde{U}^{(i-1)}(x, s)\right| \lesssim A \sup _{x \in \mathbb{R}}\left|\langle x\rangle U^{(i-1)}(x, s)\right| .
$$

We proceed to estimate the fringe integral over the interval $[t-1, t]$. The estimate here is even simpler: we get

$$
\int_{t-1}^{t}(t-s)^{-\frac{1}{2}}\left\|P_{s}\left(\alpha_{T_{i}}^{(i-1)}\right)\left[i \dot{\tilde{\pi}}^{*} \partial_{\pi} \tilde{W}_{T_{i}}\left(\pi^{(i-1)}\right)(s)\right]\right\|_{L_{x}^{1}} d s \lesssim C \delta\langle t\rangle^{-2-\epsilon} M\left(A, C, C_{0}, \delta\right) .
$$


Next, we have

$$
\begin{aligned}
& \int_{t-1}^{t}(t-s)^{-\frac{1}{2}}\left\|P_{s}\left(\alpha_{T_{i}}^{(i-1)}\right)\left[V^{(i-1)}\left(U_{d i s}^{*}+U_{h y p}^{*}+U_{\text {root }}^{*}\left(U_{d i s}^{*}, U_{\text {hyp }}^{*}\right)\right)(s)\right]\right\|_{L_{x}^{1}} d s \\
& \lesssim C \delta M\left(A, C, C_{0}, \delta\right)\langle t\rangle^{-1-\epsilon} .
\end{aligned}
$$

Of course we exploit here that

$$
\left\|\langle x\rangle^{-\theta} U^{*}\right\|_{L_{x}^{\infty}} \leq\langle t\rangle^{-1-\epsilon} M\left(A, C, C_{0}, \delta\right) .
$$

The remaining terms are more of the same and are omitted 12 . We still need to estimate the free contribution. First, note that for $0 \leq t \leq 1$ we have by Sobolev's inequality as well as the approximate unitarity of the free evolution (see Corollary 7.2

$$
\left\|\langle x\rangle^{-\theta} e^{i t \mathcal{H}\left(\alpha_{T_{i}}^{(i-1)}\right)} U_{d i s}(0)\right\|_{L_{x}^{\infty}} \lesssim\left\|e^{i t \mathcal{H}\left(\alpha_{T_{i}}^{(i-1)}\right)} U_{d i s}(0)\right\|_{H^{1}} \lesssim\|U(0)\|_{H^{1}} .
$$

The latter is estimated as in parts (A) and (C). Next, assuming $t \geq 1$, we have (using Lemma 8.2)

$$
\left\|\langle x\rangle^{-\theta} e^{i t \mathcal{H}\left(\alpha_{T_{i}}^{(i-1)}\right)} U_{d i s}(0)\right\|_{L_{x}^{\infty}} \lesssim\langle t\rangle^{-1-\epsilon}\left\|\langle x\rangle^{\theta} U_{d i s}(0)\right\|_{L_{x}^{1}} .
$$

Using that

$$
\begin{aligned}
& U_{\text {dis }}(0)= \\
& P_{s}\left(\alpha_{T_{i}}^{(i-1)}\right)\left\{\mathcal { G } _ { T _ { i } } ( \pi ^ { ( i - 1 ) } ( 0 ) ) \left[\left(\frac{R_{0}}{R_{0}}\right)+h^{(i)}\left(U_{d i s}^{*}, U_{\text {hyp }}^{*}, U^{(i-1)}, \pi^{(i-1)}, \pi^{(i-2)}\right) f^{+}\left(\alpha_{T_{i}}^{(i-1)}\right)\right.\right. \\
& \left.\left.\quad+\sum_{j=1}^{4} a_{j}^{(i)}\left(h^{(i)}\left(U_{d i s}^{*}, U_{\text {hyp }}^{*}, U^{(i-1)}, \pi^{(i-1)}, \pi^{(i-2)}\right), \alpha_{T_{i}}^{(i-1)}\right) \tilde{\eta}_{j}\left(\alpha_{T_{i}}^{(i-1)}\right)\right]\right\} .
\end{aligned}
$$

This shows that first the following term needs to be estimated:

$$
\begin{aligned}
\left\|\langle x\rangle^{\theta} P_{s}\left(\alpha_{T_{i}}^{(i-1)}\right) \mathcal{G}_{T_{i}}\left(\pi^{(i-1)}(0)\right)\left(\frac{R_{0}}{R_{0}}\right)\right\|_{L_{x}^{1}} & \lesssim\left\|\langle x\rangle^{\theta} \mathcal{G}_{T_{i}}\left(\pi^{(i-1)}(0)\right)\left(\frac{R_{0}}{R_{0}}\right)\right\|_{L_{x}^{1}} \\
& \lesssim\left\|\langle x\rangle^{\theta}\left(\frac{R_{0}}{R_{0}}\right)\right\|_{L_{x}^{1}}
\end{aligned}
$$

which is majorized by $\frac{C_{0}}{\Lambda} \delta$ for $C_{0}$ chosen sufficiently large and large $\Lambda$. The remaining terms constituting $U_{d i s}(0)$, being local, are estimated similarly, in light of the earlier comments on $h^{(i)}(\ldots)$, etc. More precisely, one obtains

$$
\left\|\langle x\rangle^{\theta} U_{d i s}(0)\right\|_{L_{x}^{1}} \leq \frac{C_{0}}{\Lambda} \delta+C \delta M\left(A, C, C_{0}, \delta\right)+C_{3}\left[(A+1)(C \delta)^{2}+(C \delta)^{2 \sigma+1}\right]
$$

for an absolute constant $C_{3}$. We are done with (E).

(F): The estimate for

$$
\sup _{0 \leq t \leq T_{i}}\langle t\rangle^{-1-\epsilon}\left\|\langle x\rangle^{-\theta} U_{h y p}\right\|_{L_{x}^{\infty}} \text { and } \sup _{0 \leq t \leq T_{i}}\langle t\rangle^{-1-\epsilon}\left\|\langle x\rangle^{-\theta} U_{\text {root }}\right\|_{L_{x}^{\infty}} .
$$

\footnotetext{
${ }^{12}$ One has to be careful here, since the paths $\pi^{(i-1)}, \pi^{(i-2)}$ diverge a bit more past time $T_{i-1}=T_{i}-1$. Thus one needs to replace $A$ by $A+1$.
} 
The estimate follows once we establish it for $U_{h y p}$, on account of the linear dependence of $U_{\text {root }}$ on $U_{d i s}, U_{\text {hyp }}$. To see it for $U_{\text {hyp }}$, use (recall the terminology from the beginning of the proof of Proposition 4.4)

$$
\begin{gathered}
b^{+}(t)=-\int_{t}^{T_{i}} e^{-\gamma\left(\alpha_{T_{i}}^{(i-1)}\right)(s-t)} g^{+}\left(U^{(*)}, U^{(i-1)}, \pi^{(i)}, \pi^{(i-1)}, \pi^{(i-2)}\right)(t) d t \\
b^{-}(t)=e^{-t \gamma\left(\alpha_{T_{i}}^{(i-1)}\right)} b^{-}(0)+\int_{0}^{t} e^{\gamma\left(\alpha_{T_{i}}^{(i-1)}\right)(s-t)} g^{-}\left(U^{*}, U^{(i-1)}, \pi^{*}, \pi^{(i-1)}, \pi^{(i-2)}\right)(t) d t .
\end{gathered}
$$

The exponentially decaying weight accounts for the integrability of the integrands. But then the desired decay rate of $\langle t\rangle^{-1-\epsilon}$ follows easily from the preceding estimates for $g^{ \pm}(\ldots)$.

(G): The estimate for $\sup _{0 \leq t \leq T_{i}}\langle t\rangle^{-1-\epsilon}\left\|\langle x\rangle^{-\frac{1}{2}-2 \epsilon} \partial_{x} U_{d i s}(t)\right\|_{L_{x}^{q}}$. This is handled by using Corollary 8.3, in addition to Duhamel's formula. One reduces to estimating the differentiated nonlinearity, which is handled just as before, using $\langle t\rangle^{-1-\epsilon}\left\|\langle x\rangle^{-\frac{1}{2}-2 \epsilon} \partial_{x} U(t)\right\|_{L_{x}^{q}}$ instead of $\langle t\rangle^{-1-\epsilon}\left\|\langle x\rangle^{-\theta} \partial_{x} U(t)\right\|_{L_{x}^{\infty}}$ in some places. Thus one gets with $\theta\left(\frac{1}{q^{\prime}}-\frac{1}{q}\right)=1+\epsilon-\frac{1}{2}\left(\frac{1}{q^{\prime}}-\frac{1}{q}\right)$ :

$$
\begin{aligned}
& \left\|\langle x\rangle^{-\frac{1}{2}-2 \epsilon} \partial_{x} U_{d i s}(t)\right\|_{L_{x}^{q}} \lesssim\left\|\langle x\rangle^{-\frac{1}{2}-2 \epsilon} e^{i t \mathcal{H}\left(\alpha_{T_{i}}^{(i-1)}\right)} U_{d i s}(0)\right\|_{W^{1, q}} \\
& +\int_{0}^{t-1}\langle t-s\rangle^{-1-\epsilon} \|\langle x\rangle^{\theta\left(\frac{1}{q^{\prime}}-\frac{1}{q}\right)} P_{s}\left(\alpha_{T_{i}}^{(i-1)}\right)\left[i \dot{\tilde{\pi}}^{*} \partial_{\pi} \tilde{W}_{T_{i}}\left(\pi^{(i-1)}\right)\right. \\
& +V^{(i-1)}\left(U_{\text {dis }}^{*}+U_{\text {hyp }}^{*}+U_{\text {root }}^{*}\left(U_{\text {dis }}^{*}, U_{\text {hyp }}^{*}\right)\right)+N_{T_{i}}\left(M_{T_{i-1}}\left(\pi^{(i-1)}\right) \mathcal{G}_{T_{i-1}}\left(\pi^{(i-1)}\right)\right. \\
& \left.\left.\quad \times \mathcal{G}_{T_{i-2}}\left(\pi^{(i-2)}\right)^{-1} M_{T_{i-2}}\left(\pi^{(i-2)}\right)^{-1} U^{(i-1)}, \pi^{(i-1)}\right)\right](s) \|_{W^{1, q^{\prime}}} d s \\
& +\int_{t-1}^{t}(t-s)^{-\frac{1}{2}} \| P_{s}\left(\alpha_{T_{i}}^{(i-1)}\right)\left[i \dot{\tilde{\pi}}^{*} \partial_{\pi} \tilde{W}_{T_{i}}\left(\pi^{(i-1)}\right)\right. \\
& +V^{(i-1)}\left(U_{\text {dis }}^{*}+U_{h y p}^{*}+U_{\text {root }}^{*}\left(U_{d i s}^{*}, U_{h y p}^{*}\right)\right)+N_{T_{i}}\left(M_{T_{i-1}}\left(\pi^{(i-1)}\right) \mathcal{G}_{T_{i-1}}\left(\pi^{(i-1)}\right)\right. \\
& \left.\left.\times \mathcal{G}_{T_{i-2}}\left(\pi^{(i-2)}\right)^{-1} M_{T_{i-2}}\left(\pi^{(i-2)}\right)^{-1} U^{(i-1)}, \pi^{(i-1)}\right)\right](s) \|_{W^{1, q^{\prime}}} d s .
\end{aligned}
$$

In order to estimate the first summand on the right, invoke Corollary 8.3 for $t \geq 1$, and use Sobolev's embedding as well as the results of $(\mathbf{A})$ and $(\mathbf{C})$ in case $0 \leq$ $t \leq 1$. Next consider the second summand, i.e., the first big integral expression. Proceeding as in case (E), we see that the local terms can be handled just as there, since the different weight gets absorbed at no cost. For the nonlocal term, one uses similarly to case $(\mathbf{E})\left(\right.$ let $\left.\frac{1}{2+}+\frac{1}{2}=\frac{1}{q^{\prime}}\right)$

$$
\begin{aligned}
& \int_{0}^{t-1}\langle t-s\rangle^{-1-\epsilon}\left\|\langle x\rangle^{\theta\left(\frac{1}{q^{\prime}}-\frac{1}{q}\right)}\left|\tilde{U}^{(i-1)}(x, s)\right|^{2 \sigma+1}\right\|_{W^{1, q^{\prime}}} d s \\
& \lesssim \int_{0}^{t-1}\langle t-s\rangle^{-1-\epsilon}\left\|\langle x\rangle^{\theta\left(\frac{1}{q^{\prime}}-\frac{1}{q}\right)} \tilde{U}^{(i-1)}(x, s)\right\|_{L_{x}^{2+}}\left\|\tilde{U}^{(i-1)}(x, s)\right\|_{W_{x}^{1,2}\left\|\tilde{U}^{(i-1)}(x, s)\right\|_{L_{x}^{\infty}}^{2 \sigma-1} d s} \\
& \lesssim A(C \delta)^{2 \sigma+1} \int_{0}^{t-1}\langle t-s\rangle^{-1-\epsilon} s^{-\sigma+\frac{1}{2}+\theta\left(\frac{1}{q^{\prime}}-\frac{1}{q}\right)} d s \lesssim A(C \delta)^{2 \sigma+1}\langle t\rangle^{-1-\epsilon},
\end{aligned}
$$

provided we have $1+\epsilon-\frac{1}{2}\left(\frac{1}{q^{\prime}}-\frac{1}{q}\right)<\sigma-\frac{3}{2}$, which we may assume. The last integral expression is estimated similarly. 
(H): Estimating $\sup _{0 \leq t \leq T_{i}}\langle t\rangle^{2+\epsilon}|\dot{\tilde{\pi}}|(t)$. For this we of course use (4.15). The following terms need to be estimated:

$$
\begin{aligned}
& \left|\left\langle U^{*}(t), i \dot{\tilde{\pi}}^{(i-1)} \tilde{\mathcal{S}}_{\ell}\left(.+y_{T_{i}}^{(i-1)}-y^{(i-1)}\right)(t)\right\rangle\right| \\
& \lesssim\left\|U^{*}(t)\right\|_{L_{x}^{2}}\left\|\tilde{\mathcal{S}}_{\ell}\left(.+y_{T_{i}}^{(i-1)}-y^{(i-1)}\right)(t)\right\|_{L_{x}^{2}}\left|\dot{\tilde{\pi}}^{(i-1)}(t)\right| \lesssim C \delta\langle t\rangle^{-2-\epsilon} .
\end{aligned}
$$

Of course this estimate is rather crude, but it suffices for our purposes. Next, consider the expression $\left\langle N\left(\tilde{U}^{(i-1)}(t), \pi^{(i-1)}(t)\right), \tilde{\xi}_{\ell}\left(\pi^{(i-1)}(t)\right)\right\rangle$. Using Lemma 2.7 we reduce this to the following two estimates. It suffices to consider $T_{i} \geq t \geq 1$.

$$
\begin{aligned}
& \left|\left\langle\left|\tilde{U}^{(i-1)}(x, t)\right|^{2} \phi^{2 \sigma-1}\left(x+\left(y_{T_{i}}^{(i-1)}-y^{(i-1)}\right)(t)\right), \tilde{\xi}_{\ell}\left(\pi^{(i-1)}(t)\right)\right\rangle\right| \\
& \lesssim(A+1)^{2}\langle t\rangle^{1+\epsilon}\left\|\langle x\rangle^{-\theta} U^{(i-1)}\right\|_{L_{x}^{\infty}}^{2}\langle t\rangle^{-2-2 \epsilon} \lesssim((A+1) C \delta)^{2}\langle t\rangle^{-2-\epsilon}, \\
& \left|\left\langle\left|\tilde{U}^{(i-1)}(x, t)\right|^{2 \sigma+1}, \tilde{\xi}_{\ell}\left(\pi^{(i-1)}(t)\right)\right\rangle\right| \lesssim t^{-\sigma-\frac{1}{2}}\left[t^{\frac{1}{2}}\left\|\tilde{U}^{(i-1)}(x, t)\right\|_{L_{x}^{\infty}}\right]^{2 \sigma+1} \| \tilde{\xi}_{\ell}\left(\pi^{(i-1)}(t) \|_{L_{x}^{1}}\right. \\
& \lesssim\langle t\rangle^{-\sigma-\frac{1}{2}}(C \delta)^{2 \sigma+1} .
\end{aligned}
$$

Finally we have controlled all the components constituting $\|(\pi, U)\|_{X_{*}\left(\left[0, T_{i}\right]\right)}$. Gathering the preceding estimates from $(\mathbf{A})-(\mathbf{H})$, we get

$$
\|(\pi, U)\|_{X_{*}\left(\left[0, T_{i}\right]\right)} \leq \frac{C_{0}}{\tilde{\Lambda}} \delta+C \delta M\left(A, C, C_{0}, \delta\right)+\tilde{C}_{3}\left[(A+1)^{2}(C \delta)^{2}+(C \delta)^{2 \sigma+1}\right]
$$

for suitable large $\tilde{\Lambda}$ (if we choose $C_{0}$ large enough) and $\tilde{C}_{3}$ an absolute constant. We conclude that if $C$ is sufficiently large in relation to $C_{0}, \tilde{C}_{3}$ and if $\delta$ is sufficiently small in relation to $C$, as well as $C_{0}$ sufficiently large in relation to $\tilde{C}_{3}$, we get

$$
\|(\pi, U)\|_{X_{*}\left(\left[0, T_{i}\right]\right)} \leq M\left(A, C, C_{0}, \delta\right),
$$

which establishes the a priori inequality we need. Since the contraction step follows along the same lines, the map $F_{T_{i}}\left(U^{(i-1)}, \pi^{(i-1)}, \pi^{(i-2)}\right)$ has a fixed point. This is the next iterate $\left(\pi^{(i)}, U^{(i)}\right)$. This establishes Proposition 4.4.

Proof of Proposition 4.5. We need to analyze the difference equation at the level of the $Z$, which will be accomplished by transforming $Z^{(i)}-Z^{(i-1)}$ into a suitable gauge, similarly to the preceding (note, however, that we need to consider the difference of the $Z^{(i)}$, since it is easy to see that the difference of the $U^{(i)}$ cannot be controlled in a reasonable way). Starting from (4.2), etc., we arrive at the following equations, valid on $\left[0, T_{i-1}\right]$ :

$$
\begin{aligned}
& i \partial_{t}\left(Z^{(i)}-Z^{(i-1)}\right)-\mathcal{H}\left(\pi^{(i-1)}\right)\left(Z^{(i)}-Z^{(i-1)}\right) \\
& =-\left[i \dot{\tilde{\pi}}^{(i)} \partial_{\pi} W\left(\pi^{(i-1)}\right)-i \dot{\tilde{\pi}}^{(i-1)} \partial_{\pi} W\left(\pi^{(i-2)}\right)\right] \\
& \quad+\left[N\left(Z^{(i-1)}, \pi^{(i-1)}\right)-N\left(Z^{(i-2)}, \pi^{(i-2)}\right)\right]+\left[\mathcal{H}\left(\pi^{(i-2)}\right)-\mathcal{H}\left(\pi^{(i-1)}\right)\right] Z^{(i-1)} \\
& -i\left[\left\langle\dot{\pi}^{(i)} \partial_{\pi} W\left(\pi^{(i-1)}\right), \xi_{l}\left(\pi^{(i-1)}\right)\right\rangle-\left\langle\dot{\pi}^{(i-1)} \partial_{\pi} W\left(\pi^{(i-2)}\right), \xi_{l}\left(\pi^{(i-2)}\right)\right\rangle\right] \\
& =\left\langle Z^{(i)}(t), i \dot{\tilde{\pi}}^{(i-1)} \tilde{\mathcal{S}}_{\ell}\left(\pi^{(i-1)}\right)(t)\right\rangle-\left\langle N\left(Z^{(i-1)}, \pi^{(i-1)}\right), \xi_{\ell}\left(\pi^{(i-1)}\right)(t)\right\rangle \\
& -\left(\left\langle Z^{(i-1)}(t), i \dot{\tilde{\pi}}^{(i-2)} \tilde{\mathcal{S}}_{\ell}\left(\pi^{(i-2)}\right)(t)\right\rangle-\left\langle N\left(Z^{(i-2)}, \pi^{(i-2)}\right), \xi_{\ell}\left(\pi^{(i-2)}\right)(t)\right\rangle\right) .
\end{aligned}
$$

We commence by analyzing the first equation. Introduce the gauged quantity

$$
U^{(i, i-1)}:=M_{T_{i}}\left(\pi^{(i-1)}\right) \mathcal{G}_{T_{i}}\left(\pi^{(i-1)}\right)\left(Z^{(i)}-Z^{(i-1)}\right) .
$$


It satisfies the following equation:

$$
\begin{aligned}
& {\left[i \partial_{t}-\mathcal{H}\left(\alpha_{T_{i}}^{(i-1)}\right)\right] U^{(i, i-1)}} \\
& =M_{T_{i}}\left(\pi^{(i-1)}\right) \mathcal{G}_{T_{i}}\left(\pi^{(i-1)}\right)\left\{-\left[i \dot{\tilde{\pi}}^{(i)} \partial_{\pi} W\left(\pi^{(i-1)}\right)-i \dot{\tilde{\pi}}^{(i-1)} \partial_{\pi} W\left(\pi^{(i-2)}\right)\right]\right. \\
& +\left[N\left(Z^{(i-1)}, \pi^{(i-1)}\right)-N\left(Z^{(i-2)}, \pi^{(i-2)}\right)\right]+\left[\mathcal{H}\left(\pi^{(i-2)}\right)-\mathcal{H}\left(\pi^{(i-1)}\right)\right] Z^{(i-1)} \\
& \quad+\left[\mathcal{H}\left(\pi^{(i-1)}(t)-\mathcal{H}\left(\pi_{T_{i}}^{(i-1)}(t)\right)\right]\left(Z^{(i)}-Z^{(i-1)}\right)\right\} .
\end{aligned}
$$

Introduce the new norm $\|(\cdot, \cdot)\|_{\tilde{Y}([0, T])}$ as $\|(\cdot, \cdot)\|_{Y^{(i)}([0, T])}$ but with the centered weight $\left\langle x-y^{(i-1)}(t)\right\rangle$ replaced by $\langle x\rangle$. We estimate the various constituents of this norm.

(A): The contribution from $\left\|\langle t\rangle^{-1} U_{d i s}^{(i, i-1)}\right\|_{L_{t}^{\infty} L_{x}^{2}\left(\left[0, T_{i-1}\right]\right)}$. For $t \in\left[0, T_{i-1}\right]$, we shall use

$$
\begin{aligned}
\left\|\langle t\rangle^{-1} U_{d i s}^{(i, i-1)}(t)\right\|_{L_{x}^{2}} \lesssim & \langle t\rangle^{-1}\left\|U_{d i s}^{(i, i-1)}(0)\right\|_{L_{x}^{2}} \\
& +\langle t\rangle^{-1} \| P_{s}\left(\alpha_{T_{i}}^{(i-1)}[\text { left-hand side of (4.17) }] \|_{L_{t}^{1} L_{x}^{2}([0, t])} .\right.
\end{aligned}
$$

We need to estimate the following terms:

$$
\begin{aligned}
& i \dot{\tilde{\pi}}^{(i)} \partial_{\pi} W\left(\pi^{(i-1)}\right)-i \dot{\tilde{\pi}}^{(i-1)} \partial_{\pi} W\left(\pi^{(i-2)}\right) \\
& =i\left(\dot{\tilde{\pi}}^{(i)}-\dot{\tilde{\pi}}^{(i-1)}\right) \partial_{\pi} W\left(\pi^{(i-1)}\right)+\dot{\tilde{\pi}}^{(i-1)}\left(\partial_{\pi} W\left(\pi^{(i-1)}\right)-\partial_{\pi} W\left(\pi^{(i-2)}\right)\right) .
\end{aligned}
$$

As for the a priori estimates, we can estimate

$$
\begin{aligned}
\| i\left(\dot{\tilde{\pi}}^{(i)}-\right. & \left.\dot{\tilde{\pi}}^{(i-1)}\right) P_{s}\left[M_{T_{i}}\left(\pi^{(i-1)}\right) \mathcal{G}_{T_{i}}\left(\pi^{(i-1)}\right) \partial_{\pi} W\left(\pi^{(i-1)}\right)\right] \|_{L_{t}^{1} L_{x}^{2}([0, t])} \\
= & \| i\left(\dot{\tilde{\pi}}^{(i)}-\dot{\tilde{\pi}}^{(i-1)}\right) \\
& \times P_{s}\left[M_{T_{i}}\left(\pi^{(i-1)}\right) \mathcal{G}_{T_{i}}\left(\pi^{(i-1)}\right)\left(\partial_{\pi} W\left(\pi^{(i-1)}\right)-\partial_{\pi} W\left(\pi_{T_{i}}^{(i-1)}\right)\right)\right] \|_{L_{t}^{1} L_{x}^{2}([0, t])} \\
\lesssim C \delta & \sup _{t \in\left[0, T_{i-1}\right]}\langle t\rangle^{1+\frac{\epsilon}{2}}\left|\left(\dot{\tilde{\pi}}^{(i)}-\dot{\tilde{\pi}}^{(i-1)}\right)(t)\right| \int_{0}^{T_{i-1}}\langle t\rangle^{-1-\frac{\epsilon}{2}} d t .
\end{aligned}
$$

Moreover, we have for $t \in\left[0, T_{i-1}\right]$

$$
\begin{aligned}
& \left\|P_{s}\left[M_{T_{i}}\left(\pi^{(i-1)}\right) \mathcal{G}_{T_{i}}\left(\pi^{(i-1)}\right) \dot{\tilde{\pi}}^{(i-1)}\left(\partial_{\pi} W\left(\pi^{(i-1)}\right)-\partial_{\pi} W\left(\pi^{(i-2)}\right)\right)\right]\right\|_{L_{t}^{1} L_{x}^{2}[0, t]} \\
& \lesssim\left(\sup _{s \in\left[0, T_{i-1}\right]}\left\langle s^{2+\epsilon}\right\rangle\left|\dot{\pi}^{(i-1)}\right|(s)\right) \int_{0}^{t}\langle s\rangle^{-1-\frac{\epsilon}{2}} d s \sup _{s \in\left[0, T_{i-1}\right]}\left|\tilde{\pi}^{(i-1)}-\tilde{\pi}^{(i-2)}\right|(s) \\
& \lesssim C \delta \sup _{s \in\left[0, T_{i-1}\right]}\langle s\rangle^{1+\frac{\epsilon}{2}}\left|\dot{\tilde{\pi}}^{(i-1)}-\dot{\tilde{\pi}}^{(i-2)}\right|(s) .
\end{aligned}
$$

We have used the following simple lemma.

Lemma 4.8. The following inequality holds for $t \in\left[0, T_{i-1}\right]$ :

$$
\sup _{s \in[0, t]}\left|\partial_{\pi} W\left(\pi^{(i-1)}\right)(s)-\partial_{\pi} W\left(\pi^{(i-2)}\right)(s)\right| \lesssim \sup _{s \in[0, t]}\langle s\rangle^{1+\frac{\epsilon}{2}}\left|\tilde{\pi}^{(i-1)}(s)-\tilde{\pi}^{(i-2)}(s)\right| .
$$

Proof. Note that $\partial_{\pi} W\left(\pi^{(i-1)}\right)$ consists precisely of the generalized root functions, translated by $y^{(i-1)}$ and twisted by a phase $e^{i \theta^{(i-1)}}$, and similarly for $\partial_{\pi} W\left(\pi^{(i-2)}\right)$. 
But we have for $s \in[0, t]$

$$
\begin{aligned}
& \left|y^{(i-1)}(s)-y^{(i-2)}(s)\right| \leq \int_{0}^{s}\left|v^{(i-1)}\left(s^{\prime}\right)-v^{(i-2)}\left(s^{\prime}\right)\right| d s^{\prime}+\left|D^{(i-1)}(s)-D^{(i-2)}(s)\right| \\
& \lesssim \sup _{s \in[0, t]}\langle s\rangle^{1+\frac{\epsilon}{2}}\left|v^{(i-1)}(s)-v^{(i-2)}(s)\right|+\left|D^{(i-1)}(s)-D^{(i-2)}(s)\right|
\end{aligned}
$$

and similarly, we have

$$
\begin{aligned}
& \left|\theta^{(i-1)}(s, x)-\theta^{(i-2)}(s, x)\right| \\
& \leq\left|v^{(i-1)}(s)-v^{(i-2)}(s)\right||x|+\left|\int_{0}^{s}\left(\left(v^{(i-1)}\right)^{2}\left(s^{\prime}\right)-\left(v^{(i-2)}\left(s^{\prime}\right)\right)^{2}\right) d s^{\prime}\right| \\
& +\left|\int_{0}^{s}\left(\left(\alpha^{(i-1)}\right)^{2}\left(s^{\prime}\right)-\left(\alpha^{(i-2)}\left(s^{\prime}\right)\right)^{2}\right) d s^{\prime}\right|+\left|\gamma^{(i-1)}(s)-\gamma^{(i-2)}(s)\right| \\
& \lesssim\left|v^{(i-1)}(s)-v^{(i-2)}(s)\right||x|+\left|\gamma^{(i-1)}(s)-\gamma^{(i-2)}(s)\right| \\
& +\sup _{s \in[0, t]}\langle s\rangle^{1+\frac{\epsilon}{2}}\left[\left|v^{(i-1)}(s)-v^{(i-2)}(s)\right|+\left|\alpha^{(i-1)}(s)-\alpha^{(i-2)}(s)\right|\right] \\
& +\left|\gamma^{(i-1)}(s)-\gamma^{(i-2)}(s)\right| .
\end{aligned}
$$

Of course the factor $x$ gets absorbed because of the local nature of the term. Finally note that

$$
\left|\gamma^{(i-1)}(s)-\gamma^{(i-2)}(s)\right| \lesssim \sup _{s^{\prime} \in[0, s]}\left\langle s^{\prime}\right\rangle^{1+\frac{\epsilon}{2}}\left|\tilde{\pi}^{(i-1)}-\tilde{\pi}^{(i-2)}\right|\left(s^{\prime}\right),
$$

and the lemma follows.

In order to estimate the second term in the nonlinearity, we use that

$$
\begin{aligned}
& {\left[N\left(Z^{(i-1)}, \pi^{(i-1)}\right)-N\left(Z^{(i-2)}, \pi^{(i-2)}\right)\right]} \\
& =\left[Z^{(i-1)}-Z^{(i-2)}\right] \int_{0}^{1} \partial_{Z} N\left(s\left[Z^{(i-1)}-Z^{(i-2)}\right]+Z^{(i-2)}, \pi^{(i-1)}\right) d s \\
& +\left[\pi^{(i-1)}-\pi^{(i-2)}\right] \int_{0}^{1} \partial_{\pi} N\left(Z^{(i-2)}, \pi^{(i-2)}+s\left(\pi^{(i-1)}-\pi^{(i-2)}\right)\right) d s .
\end{aligned}
$$

Now we have for $t \in\left[0, T_{i-1}\right]$

$$
\begin{aligned}
& \langle t\rangle^{-1} \| P_{s}\left[M_{T_{i}}\left(\pi^{(i-1)}\right) \mathcal{G}_{T_{i}}\left(\pi^{(i-1)}\right)\left[Z^{(i-1)}-Z^{(i-2)}\right]\right. \\
& \left.\quad \times \int_{0}^{1} \partial_{Z} N\left(s\left[Z^{(i-1)}-Z^{(i-2)}\right]+Z^{(i-2)}, \pi^{(i-1)}\right) d s\right] \|_{L_{t}^{1} L_{x}^{2}[0, t]} \\
& \lesssim\left[A C \delta+(C \delta)^{2 \sigma}\right] \sup _{s \in\left[0, T_{i-1}\right]}\langle s\rangle^{-1}\left\|Z^{(i-1)}-Z^{(i-2)}\right\|_{L_{x}^{2}}(s) .
\end{aligned}
$$

We have exploited that

$$
\begin{aligned}
& \left|\partial_{Z} N\left(s\left[Z^{(i-1)}-Z^{(i-2)}\right]+Z^{(i-2)}, \pi^{(i-1)}\right)\right| \\
& \quad \lesssim\left|s\left[Z^{(i-1)}-Z^{(i-2)}\right]+Z^{(i-2)}\right|^{2 \sigma}+\phi\left(.-y^{(i-1)}\right)\left|s\left[Z^{(i-1)}-Z^{(i-2)}\right]+Z^{(i-2)}\right|
\end{aligned}
$$


which follows from Lemma 2.7 Next, using reasoning as in the proof of Lemma 4.8 in combination with Lemma 2.7, we obtain

$$
\begin{aligned}
\mid\left[\pi^{(i-1)}-\right. & \left.\pi^{(i-2)}\right](t) \int_{0}^{1} \partial_{\pi} N\left(Z^{(i-2)}, \pi^{(i-2)}+s\left(\pi^{(i-1)}-\pi^{(i-2)}\right)\right)(t) d s \mid \\
\lesssim & \left.\sup _{s \in[0, t]}\langle s\rangle^{1+\frac{\epsilon}{2}}\left|\tilde{\pi}^{(i-1)}-\tilde{\pi}^{(i-2)}\right|(s)\right]\left|Z^{(i-2)}(t)\right|^{2} \\
& \quad \times \sup _{0 \leq s \leq 1} \phi\left(.-y^{(i-1)}+s\left(y^{(i-1)}-y^{(i-2)}\right)\right)(t) .
\end{aligned}
$$

We can further estimate this by

$$
\begin{aligned}
\mid\left[\sup _{s \in[0, t]}\langle\right. & \left.\langle s\rangle^{1+\frac{\epsilon}{2}}\left|\tilde{\pi}^{(i-1)}-\tilde{\pi}^{(i-2)}\right|(s)\right]\left|Z^{(i-2)}(t)\right|^{2} \\
& \times \sup _{0 \leq s \leq 1} \phi\left(.-y^{(i-1)}+s\left(y^{(i-1)}-y^{(i-2)}\right)\right)(t) \mid \\
\lesssim & \langle t\rangle^{1+\frac{\epsilon}{2}}\left[\left\langle x-y^{(i-1)}\right\rangle^{-\theta}\left|Z^{(i-2)}\right|(t)\right]^{2} \\
& \times \sup _{0 \leq s \leq 1}\left\langle x-y^{(i-1)}\right\rangle^{2 \theta} \phi\left(.-y^{(i-1)}+s\left(y^{(i-1)}-y^{(i-2)}\right)\right)(t) \\
& \times \sup _{0 \leq s \leq T_{i-1}}\left|\tilde{\pi}^{(i-1)}-\tilde{\pi}^{(i-2)}\right|(s)
\end{aligned}
$$

and we bound this in turn by

$$
\lesssim(A C \delta)^{2}\langle t\rangle^{-1-\frac{\epsilon}{2}} \sup _{0 \leq s \leq T_{i-1}}\langle s\rangle^{1+\frac{\epsilon}{2}}\left|\dot{\tilde{\pi}}^{(i-1)}-\dot{\tilde{\pi}}^{(i-2)}\right|(s),
$$

which can be comfortably integrated against $t$. Now we estimate the third term in the nonlinearity. Note that it is here that we are forced to use the extra weight $\langle t\rangle^{-1}$ :

$$
\begin{aligned}
& \langle t\rangle^{-1}\left\|P_{s}\left\{M_{T_{i}}\left(\pi^{(i-1)}\right) \mathcal{G}_{T_{i}}\left(\pi^{(i-1)}\right)\left[\mathcal{H}\left(\pi^{(i-2)}\right)-\mathcal{H}\left(\pi^{(i-1)}\right)\right] Z^{(i-1)}\right\}\right\|_{L_{t}^{1} L_{x}^{2}[0, t]} \\
& \lesssim A C \delta \sup _{s \in\left[0, T_{i-1}\right]}\left|\tilde{\pi}^{(i-1)}-\tilde{\pi}^{(i-2)}\right|(s) .
\end{aligned}
$$

Once again we use a version of Lemma 4.8. Furthermore, we obtain

$$
\begin{aligned}
& \sup _{0 \leq t \leq T_{i-1}}\langle t\rangle^{-1}\left\|\left[\mathcal{H}\left(\pi^{(i-1)}(\cdot)\right)-\mathcal{H}\left(\pi_{T_{i}}^{(i-1)}(\cdot)\right)\right]\left(Z^{(i)}-Z^{(i-1)}\right)\right\|_{L_{t}^{1} L_{x}^{2}[0, t]} \\
& \lesssim C \delta \sup _{0 \leq s \leq T_{i-1}}\left\|\left\langle\cdot-y^{(i-1)}(s)\right\rangle^{-\theta}\left(Z^{(i)}-Z^{(i-1)}\right)(s)\right\|_{L_{x}^{\infty} .}
\end{aligned}
$$

Next, consider $\langle t\rangle^{-1}\left\|U_{d i s}^{(i, i-1)}(0)\right\|_{L_{x}^{2}}$. Note that

$$
\begin{aligned}
& U^{(i, i-1)}(0) \\
& \quad=U^{i}(0)-M_{T_{i}}\left(\pi^{(i-1)}\right) \mathcal{G}_{T_{i}}\left(\pi^{(i-1)}\right)(0) \mathcal{G}_{T_{i-1}}^{-1}\left(\pi^{(i-2)}\right)(0) M_{T_{i}}^{-1}\left(\pi^{(i-2)}\right) U^{(i-1)}(0) .
\end{aligned}
$$


Moreover, we have

$$
\begin{aligned}
U^{(i)}(0)= & M_{T_{i}}\left(\pi^{(i-1)}\right) \mathcal{G}_{T_{i}}\left(\pi^{(i-1)}\right)(0) \\
& \times\left[\left(\begin{array}{c}
R_{0} \\
\bar{R}_{0}
\end{array}\right)+h^{(i)} f^{+}\left(\alpha_{T_{i}}^{(i-1)}\right)+\sum_{k=1}^{4} a_{k}^{(i)} \tilde{\eta}_{k}\left(\alpha_{T_{i}}^{(i-1)}\right)\right], \\
U^{(i-1)}(0)= & M_{T_{i-1}}\left(\pi^{(i-2)}\right) \mathcal{G}_{T_{i-1}}\left(\pi^{(i-2)}\right)(0) \\
\times & {\left[\left(\begin{array}{c}
R_{0} \\
\bar{R}_{0}
\end{array}\right)+h^{(i-1)} f^{+}\left(\alpha_{T_{i-1}}^{(i-2)}\right)+\sum_{k=1}^{4} a_{k}^{(i-1)} \tilde{\eta}_{k}\left(\alpha_{T_{i-1}}^{(i-2)}\right)\right] . }
\end{aligned}
$$

The conclusion is that

$$
U^{(i, i-1)}(0)=O\left(\delta\left[\tilde{\pi}_{T_{i}}^{(i-1)}-\tilde{\pi}_{T_{i-1}}^{(i-2)}\right]\right)+O\left(h^{(i)}-h^{(i-1)}\right) .
$$

Thus we need to estimate the difference $h^{(i)}-h^{(i-1)}$. For this, we decompose $U^{(i, i-1)}$ with respect to the operator $\mathcal{H}\left(\alpha_{T_{i}}^{(i-1)}\right)$ :

$$
\begin{aligned}
U^{(i, i-1)} & =\sum_{j=1}^{4} \tilde{a}_{j}^{(i, i-1)}(t) \tilde{\eta}_{j}^{(i-1)}\left(\alpha_{T_{i}}^{(i-1)}\right)+b^{+(i, i-1)}(t) f^{+}\left(\alpha_{T_{i}}^{(i-1)}\right) \\
& +b^{-(i, i-1)}(t) f^{-}\left(\alpha_{T_{i}}^{(i-1)}\right)+U_{d i s}^{(i, i-1)}
\end{aligned}
$$

In other words, we have

$$
\begin{aligned}
U_{\text {root }}^{(i, i-1)} & =\sum_{j=1}^{4} \tilde{a}_{j}^{(i, i-1)}(t) \tilde{\eta}_{j}^{(i-1)}\left(\alpha_{T_{i}}^{(i-1)}\right), \\
U_{\text {hyp }}^{(i, i-1)} & =b^{+(i, i-1)}(t) f^{+}\left(\alpha_{T_{i}}^{(i-1)}\right)+b^{-(i, i-1)}(t) f^{-}\left(\alpha_{T_{i}}^{(i-1)}\right) .
\end{aligned}
$$

In particular, we have the identity

$$
\begin{aligned}
& b^{(+)(i, i-1)}(0) f^{+}\left(\alpha_{T_{i}}^{(i-1)}\right) \\
& =P_{I m}^{+}\left(\alpha_{T_{i}}^{(i-1)}\right)\left\{M _ { T _ { i } } ( \pi ^ { ( i - 1 ) } ) ( 0 ) \mathcal { G } _ { T _ { i } } ( \pi ^ { ( i - 1 ) } ) ( 0 ) \left[h^{(i)} f^{+}\left(\alpha_{T_{i}}^{(i-1)}\right)-h^{(i-1)} f^{+}\left(\alpha_{T_{i}}^{(i-2)}\right)\right.\right. \\
& \left.\left.\quad+\sum_{k=1}^{4}\left(a_{k}^{(i)}-a_{k}^{(i-1)}\right) \tilde{\eta}_{k}\left(\alpha_{T_{i}}^{(i-1)}\right)+\sum_{k} a_{k}^{(i-1)}\left[\tilde{\eta}_{k}\left(\alpha_{T_{i}}^{(i-1)}\right)-\tilde{\eta}_{k}\left(\alpha_{T_{i}}^{(i-2)}\right)\right]\right]\right\} .
\end{aligned}
$$

In order to control the difference $h^{(i)}-h^{(i-1)}$, we need to control $b^{(+)(i, i-1)}(0)$. Indeed, assume we control the latter. Observe the simple identity

$$
\begin{aligned}
h^{(i)} f^{+}\left(\alpha_{T_{i}}^{(i-1)}\right)-h^{(i-1)} f^{+}\left(\alpha_{T_{i-1}}^{(i-2)}\right) & =\left[h^{(i)}-h^{(i-1)}\right] f^{+}\left(\alpha_{T_{i}}^{(i-1)}\right) \\
& +h^{(i-1)}\left[f^{+}\left(\alpha_{T_{i}}^{(i-1)}\right)-f^{+}\left(\alpha_{T_{i-1}}^{(i-2)}\right)\right] .
\end{aligned}
$$

The conclusion is that 13

$$
\begin{aligned}
& \left|h^{(i)}-h^{(i-1)}\right| \\
& \lesssim\left|P_{I m}^{+}\left(\alpha_{T_{i}}^{(i-1)}\right)\left\{M_{T_{i}}\left(\pi^{(i-1)}\right)(0) \mathcal{G}_{T_{i}}\left(\pi^{(i-1)}\right)(0)\left[h^{(i)} f^{+}\left(\alpha_{T_{i}}^{(i-1)}\right)-h^{(i-1)} f^{+}\left(\alpha_{T_{i-1}}^{(i-2)}\right)\right]\right\}\right| \\
& +\left|P_{I m}^{+}\left(\alpha_{T_{i}}^{(i-1)}\right)\left\{M_{T_{i}}\left(\pi^{(i-1)}\right)(0)\left(\mathcal{G}_{T_{i}}\left(\pi^{(i-1)}\right)-I\right)(0)\left[h^{(i)} f^{+}\left(\alpha_{T_{i}}^{(i-1)}\right)-h^{(i-1)} f^{+}\left(\alpha_{T_{i-1}}^{(i-2)}\right)\right]\right\}\right| \\
& +\delta\left|\alpha_{T_{i}}^{(i-1)}-\alpha_{T_{i-1}}^{(i-2)}\right| .
\end{aligned}
$$

\footnotetext{
${ }^{13}$ Use that $M_{T_{i}}\left(\pi^{(i-1)}\right)(0)=I$.
} 
On account of the a priori estimates established thus far, we conclude that

$$
\left|\left(\mathcal{G}_{T_{i}}\left(\pi^{(i-1)}\right)(0)-I\right) f^{ \pm}\left(\alpha_{T_{i}}^{(i-1)}\right)\right| \lesssim C \delta .
$$

Furthermore, back in (4.19), we have

$$
\begin{aligned}
& \left|P_{I m}^{+}\left(\alpha_{T_{i}}^{(i-1)}\right)\left[M_{T_{i}}\left(\pi^{(i-1)}\right) \mathcal{G}_{T_{i}}\left(\pi^{(i-1)}\right) \sum_{k=1}^{4}\left(a_{k}^{(i)}-a_{k}^{(i-1)}\right) \tilde{\eta}_{k}\left(\alpha_{T_{i}}^{(i-1)}\right)\right]\right|(0) \\
& =\left|P_{I m}^{+}\left(\alpha_{T_{i}}^{(i-1)}\right)\left[\left(M_{T_{i}}\left(\pi^{(i-1)}\right) \mathcal{G}_{T_{i}}\left(\pi^{(i-1)}\right)-I\right) \sum_{k=1}^{4}\left(a_{k}^{(i)}-a_{k}^{(i-1)}\right) \tilde{\eta}_{k}\left(\alpha_{T_{i}}^{(i-1)}\right)\right]\right|(0) \\
& \lesssim \delta\left[\left|h^{(i)}-h^{(i-1)}\right|+\left|\alpha_{T_{i}}^{(i-1)}-\alpha_{T_{i-1}}^{(i-2)}\right|\right] .
\end{aligned}
$$

We use here that $a_{k}^{(i)}=a_{k}^{(i-1)}\left(h^{(i)}, \alpha_{T_{i}}^{(i-1)}\right)$, etc. Also, we observe that 14

$$
\begin{aligned}
& \left|P_{I m}^{+}\left(\alpha_{T_{i}}^{(i-1)}\right)\left[M_{T_{i}}\left(\pi^{(i-1)}\right) \mathcal{G}_{T_{i}}\left(\pi^{(i-1)}\right) \sum_{k} a_{k}^{(i-1)}\left(\tilde{\eta}_{k}\left(\alpha_{T_{i}}^{(i-1)}\right)-\tilde{\eta}_{k}\left(\alpha_{T_{i-1}}^{(i-2)}\right)\right)\right]\right| \\
& \quad \lesssim \delta\left|\tilde{\pi}_{T_{i}}^{(i-1)}-\tilde{\pi}_{T_{i-1}}^{(i-2)}\right| .
\end{aligned}
$$

Finally, we observe that thanks to the a priori estimates thus far established $\left|\tilde{\pi}_{T_{i}}^{(i-1)}-\tilde{\pi}_{T_{i-1}}^{(i-2)}\right| \leq\left|\tilde{\pi}_{T_{i-1}}^{(i-1)}-\tilde{\pi}_{T_{i-1}}^{(i-2)}\right|+\left|\dot{\tilde{\pi}}^{(i-1)}\right| \lesssim \sup _{t \in\left[0, T_{i-1}\right]}\left|\tilde{\pi}^{(i-1)}-\tilde{\pi}^{(i-2)}\right|(t)+T_{i-1}^{-2-\epsilon}$.

Putting all of these observations together and using (4.19), we get that

$$
\begin{aligned}
& \left|h^{(i)}-h^{(i-1)}\right| \\
& \quad \lesssim b^{+(i, i-1)}(0)+\delta\left\|\left(\tilde{\pi}^{(i-1)}-\tilde{\pi}^{(i-2)}, Z^{(i-1)}-Z^{(i-2)}\right)\right\|_{Y^{(i-1)}\left(\left[0, T_{i-1}\right]\right)}+T_{i-1}^{-2-\epsilon}
\end{aligned}
$$

and thus it suffices to estimate $b^{+(i, i-1)}(0)$, as claimed above. To do so, we observe the ODE system satisfied by the $b^{+(i, i-1)}$ as follows:

$$
\begin{aligned}
& \frac{d}{d t}\left(\begin{array}{c}
b^{(i, i-1)+}(t) \\
b^{(i, i-1)-}(t)
\end{array}\right)-\left(\begin{array}{cc}
\gamma\left(\alpha_{T_{i}}^{(i-1)}\right) & 0 \\
0 & -\gamma\left(\alpha_{T_{i}}^{(i-1)}\right)
\end{array}\right)\left(\begin{array}{c}
b^{(i, i-1)+}(t) \\
b^{(i, i-1)-}(t)
\end{array}\right) \\
& =\left(\begin{array}{c}
g^{+}\left(U^{(i, i-1)}, U^{(i-1)}, U^{(i-2)}, \pi^{(i-1)}, \pi^{(i-2)}, \dot{\pi}^{(i)}-\dot{\pi}^{(i-1)}\right) \\
g^{-}\left(U^{(i, i-1)} U^{(i-1)}, U^{(i-2)}, \pi^{(i-1)}, \pi^{(i-2)}, \pi^{(i)}-\pi^{(i-1)}\right)
\end{array}\right)
\end{aligned}
$$

where

$$
\begin{aligned}
g^{ \pm}\left(U^{(i, i-1)}, U^{(i-1)}, \pi^{(i-1)}, \pi^{(i-2)}, \dot{\pi}^{(i)}-\dot{\pi}^{(i-1)}\right) & \\
=P_{I m}^{ \pm}\left(\alpha_{T_{i}}^{(i-1)}\right)\left\{M_{T_{i}}\left(\pi^{(i-1)}\right) \mathcal{G}_{T_{i}}\left(\pi^{(i-1)}\right)[\right. & \text { right-hand side of } \\
& \left.\left.+V^{(i-1)}\left(Z^{(i)}-Z^{(i-1)}\right)\right]\right\} .
\end{aligned}
$$

On the other hand, by construction of $U^{(i, i-1)}$ as the difference of two $L^{2}$-bounded functions, we know that $b^{(i, i-1)}(t)$ is controlled by an a priori bound $C \delta$ on $\left[0, T_{i}\right]$. The solution $b^{+(i, i-1)}(t)$ of the ODE is given by

$$
b^{+(i, i-1)}(t)=e^{t \gamma\left(\alpha_{T_{i}}^{(i-1)}\right)} b^{+(i, i-1)}(0)+\int_{0}^{t} e^{(t-s) \gamma\left(\alpha_{T_{i}}^{(i-1)}\right)} g^{+}(,) d s .
$$

${ }^{14}$ Use that $a_{k}^{(i)}=O(\delta)$. 
We conclude that

$$
b^{+(i, i-1)}(0)=-\int_{0}^{T_{i-1}} e^{-s \gamma\left(\alpha_{T_{i}}^{(i-1)}\right)} g^{+}(,) d s+O\left(e^{-\gamma\left(\alpha_{T_{i}}^{(i-1)}\right) T_{i-1}}\right) .
$$

Thus we need to estimate $g^{+}($,$) . However, this can be done as in our previous$ work concerning the bound on $\left\|\langle t\rangle^{-1}\left(Z^{(i)}-Z^{(i-1)}\right)\right\|_{L_{x}^{2}}$. This completes estimating the dispersive part $U_{d i s}^{(i, i-1)}$.

(B): We also need to estimate the root as well as the hyperbolic parts for $t \in$ $\left[0, T_{i-1}\right]$. For the latter, one again invokes the ODE system, as before. However, there is a technical difficulty since we are no longer working on an infinite interval and can no longer conclude that there is just one possible value for $b^{+(i, i-1)}(0)$ given in terms of an integral representation. We do have the representation (for $\left.t \in\left[0, T_{i-1}\right]\right)$

$$
b^{+(i, i-1)}(t)=e^{t \gamma\left(\alpha_{T_{i}}^{(i-1)}\right)} b^{+(i, i-1)}(0)+\int_{0}^{t} e^{(t-s) \gamma\left(\alpha_{T_{i}}^{(i-1)}\right)} g^{+}(,) d s .
$$

As before, this allows us to conclude that

$$
b^{+(i, i-1)}(t)=O\left(e^{\left(t-T_{i-1}\right) \gamma\left(\alpha_{T_{i}}^{(i-1)}\right)}\right)-\int_{t}^{T_{i-1}} e^{(t-s) \gamma\left(\alpha_{T_{i}}^{(i-1)}\right)} g^{+}(,) d s .
$$

The first term on the left is only $O(1)$, though, as $t \rightarrow T_{i-1}$. Note that this problem does not occur for the establishment of the a priori estimates since we define $b^{+(i)}(0)$, etc., to be given by the integral expression. To remedy this, observe that we also have

$$
\begin{aligned}
& U^{(i, i-1)}(t)=U^{(i)}(t) \\
& -M_{T_{i}}\left(\pi^{(i-1)}\right)(t) \mathcal{G}_{T_{i}}\left(\pi^{(i-1)}\right)(t) \mathcal{G}_{T_{i-1}}\left(\pi^{(i-2)}\right)(t)^{-1} M_{T_{i-1}}\left(\pi^{(i-2)}\right)(t)^{-1} U^{(i-1)}(t) .
\end{aligned}
$$

Observe that as long as $T_{i-1}-t \geq C \log \left|T_{i-1}\right|$, one obtains the desired estimate for $b^{(i, i-1)}(t)$. If $T_{i-1}-t<C \log \left|T_{i-1}\right|$, one invokes the a priori estimates, which produce a bound of the form

$$
\langle t\rangle^{-1}\left\|U_{h y p}^{(i, i-1)}(t)\right\|_{L_{x}^{2}} \lesssim\langle t\rangle^{-1}\left[\left\|U^{(i)}(t)\right\|_{L_{x}^{\infty}}+\left\|U^{(i-1)}(t)\right\|_{L_{x}^{\infty}} \lesssim C \delta T_{i-1}^{-\frac{3}{2}},\right.
$$

which is more than what we need. We proceed to the root part. Using the notation from above, we have

$$
U_{\text {root }}^{(i, i-1)}(t)=\sum_{j=1}^{4} \tilde{a}_{j}^{(i, i-1)}(t) \tilde{\eta}_{j}^{(i-1)}\left(\alpha_{T_{i}}^{(i-1)}\right) .
$$

From the iterative construction, we have

$$
\left\langle U^{(i, i-1)}, \tilde{\xi}_{\ell}\left(\pi^{(i-1)}\right)\right\rangle=\left\langle Z^{(i, i-1)}, \xi_{\ell}\left(\pi^{(i-1)}\right)\right\rangle=\left\langle Z^{(i-1)},-\xi_{\ell}\left(\pi^{(i-2)}\right)+\xi_{\ell}\left(\pi^{(i-1)}\right)\right\rangle .
$$

Hence

$$
\begin{aligned}
\left\langle U_{\text {root }}^{(i, i-1)}, \tilde{\xi}_{\ell}\left(\pi^{(i-1)}\right)\right\rangle= & -\left\langle U_{\text {dis }}^{(i, i-1)}, \tilde{\xi}_{\ell}\left(\pi^{(i-1)}\right)\right\rangle \\
& -\left\langle U_{\text {hyp }}^{(i, i-1)}, \tilde{\xi}_{\ell}\left(\pi^{(i-1)}\right)\right\rangle+\left\langle Z^{(i-1)},-\xi_{\ell}\left(\pi^{(i-2)}\right)+\xi_{\ell}\left(\pi^{(i-1)}\right)\right\rangle .
\end{aligned}
$$

From here one obtains the bound

$$
\begin{aligned}
& \sup _{t \in\left[0, T_{i-1}\right]}\langle t\rangle^{-1}\left\|U_{\text {root }}^{(i, i-1)}(t)\right\|_{L_{x}^{2}} \\
& \lesssim A C \delta\left\|\left(Z^{(i-1)}-Z^{(i-2)}, \pi^{(i-1)}-\pi^{(i-2)}\right)\right\|_{Y^{(i-1)}\left[0, T_{i-1}\right]}+C \delta T_{i}^{-1-\epsilon} .
\end{aligned}
$$


(C): Finally, we consider $\sup _{t \in\left[T_{i-1}, T_{i}\right]}\langle t\rangle^{-1}\left\|U^{(i, i-1)}\right\|_{L_{x}^{2}}$ : we use the a priori estimates to obtain

$$
\langle t\rangle^{-1}\left\|U^{(i, i-1)}\right\| \leq\langle t\rangle^{-1}\left[\left\|Z^{(i)}\right\|_{L_{x}^{2}}+\left\|Z^{(i-1)}\right\|_{L_{x}^{2}}\right] \lesssim C \delta T_{i}^{-1} .
$$

We proceed to the weighted $L^{\infty}$-norm

$$
\sup _{t \in\left[0, T_{i}\right]}\left\|\left\langle x-y^{(i-1)}(t)\right\rangle^{-\theta}\left(Z^{(i)}-Z^{(i-1)}\right)(t)\right\|_{L_{x}^{\infty}} .
$$

We follow the same strategy as before by changing the gauge, working with

$$
U^{(i, i-1)}=M_{T_{i}}\left(\pi^{(i-1)}\right)(t) \mathcal{G}_{T_{i}}\left(\pi^{(i-1)}\right)(t)\left(Z^{(i)}-Z^{(i-1)}\right)(t) .
$$

(D): The estimate for $\left\|\langle x\rangle^{-\theta} U_{d i s}^{(i, i-1)}\right\|_{L_{x}^{\infty}\left(\left[0, T_{i-1}\right]\right)}$. We use (4.18) and Lemma 8.2 as well as the Duhamel parametrix to obtain

$$
\begin{aligned}
& \left\|\langle x\rangle^{-\theta} U_{d i s}^{(i, i-1)}(t)\right\|_{L_{x}^{\infty}} \lesssim\left\|\langle x\rangle^{-\theta} e^{i t \mathcal{H}\left(\alpha_{T_{i}}^{(i-1)}\right)} U_{d i s}^{(i, i-1)}(0)\right\|_{L_{x}^{\infty}} \\
& +\int_{0}^{t-1}(t-s)^{-1-\epsilon} \|\langle x\rangle^{\theta} P_{s}\left(\alpha_{T_{i}}^{(i-1)}\right)[\text { left-hand side of (4.18)}(s)] \|_{L_{x}^{1}} d s \\
& +\int_{t-1}^{t}(t-s)^{-\frac{1}{2}} \| P_{s}\left(\alpha_{T_{i}}^{(i-1)}\right)[\text { left-hand side of (4.18) }(s)] \|_{L_{x}^{1}} d s .
\end{aligned}
$$

We start with the first integral on the right, which can be decomposed into the following terms:

$$
\begin{aligned}
\int_{0}^{t-1}(t-s)^{-1-\epsilon} \|\langle x\rangle^{\theta} P_{s}\left(\alpha_{T_{i}}^{(i-1)}\right)\left[M_{T_{i}}\left(\pi^{(i-1)}\right)\right. & \mathcal{G}_{T_{i}}\left(\pi^{(i-1)}\right)\left[i \dot{\tilde{\pi}}^{(i)} \partial_{\pi} W\left(\pi^{(i-1)}\right)\right. \\
& \left.\left.-i \dot{\tilde{\pi}}^{(i-1)} \partial_{\pi} W\left(\pi^{(i-2)}\right)\right]\right](s) \|_{L_{x}^{1}} d s .
\end{aligned}
$$

We split this into two contributions, the first of which is

$$
\begin{aligned}
\int_{0}^{t-1}(t-s)^{-1-\epsilon} \|\langle x\rangle^{\theta} P_{s}\left(\alpha_{T_{i}}^{(i-1)}\right)\{ & M_{T_{i}}\left(\pi^{(i-1)}\right) \mathcal{G}_{T_{i}}\left(\pi^{(i-1)}\right) \\
& \left.\times\left[i\left(\dot{\tilde{\pi}}^{(i)}-\dot{\tilde{\pi}}^{(i-1)}\right) \partial_{\pi} W\left(\pi^{(i-1)}\right)\right](s)\right\} \|_{L_{x}^{1}} d s .
\end{aligned}
$$

Observe that one may move the $\langle x\rangle^{\theta}$ inside, replacing it by $\left\langle x-y^{(i-1)}(t)\right\rangle^{\theta}$. Then one may replace the expression

$$
P_{s}\left(\alpha_{T_{i}}^{(i-1)}\right)\left[M_{T_{i}}\left(\pi^{(i-1)}\right) \mathcal{G}_{T_{i}}\left(\pi^{(i-1)}\right) \partial_{\pi} W\left(\pi^{(i-1)}\right)\right]
$$

by

$$
P_{s}\left(\alpha_{T_{i}}^{(i-1)}\right)\left\{M_{T_{i}}\left(\pi^{(i-1)}\right) \mathcal{G}_{T_{i}}\left(\pi^{(i-1)}\right)\left[\partial_{\pi} W\left(\pi^{(i-1)}\right)-\partial_{\pi} W\left(\pi_{T_{i}}^{(i-1)}\right)\right]\right\}
$$

see Lemma 2.4. Thus, we need to estimate

$$
\delta \int_{0}^{t-1}(t-s)^{-1-\epsilon}\left\|\left\langle x-y^{(i-1)}(s)\right\rangle^{\theta} i\left(\dot{\tilde{\pi}}^{(i)}-\dot{\tilde{\pi}}^{(i-1)}\right)\left[\partial_{\pi} W\left(\pi^{(i-1)}\right)(s)-\partial_{\pi} W\left(\pi_{T_{i}}^{(i-1)}\right)\right]\right\|_{L_{x}^{1}} d s .
$$

Due to the local nature of this expression, one can suppress the factor $\left\langle x-y^{(i-1)}(s)\right\rangle^{\theta}$, and we can estimate the above by

$$
\begin{aligned}
& \delta \sup _{t \in\left[0, T_{i-1}\right]}\langle t\rangle^{1+\frac{\epsilon}{2}}\left|\dot{\tilde{\pi}}^{(i)}-\dot{\tilde{\pi}}^{(i-1)}\right|(t) \int_{0}^{t-1}(t-s)^{-1-\epsilon}\langle s\rangle^{-1-\frac{\epsilon}{2}} d s \\
& \lesssim C \delta \sup _{t \in\left[0, T_{i-1}\right]}\langle t\rangle^{1+\frac{\epsilon}{2}}\left|\dot{\tilde{\pi}}^{(i)}-\dot{\tilde{\pi}}^{(i-1)}\right|(t) .
\end{aligned}
$$


The second contribution is the following:

$$
\begin{aligned}
& \int_{0}^{t-1}(t-s)^{-1-\epsilon} \|\langle x\rangle^{\theta} P_{s}\left(\alpha_{T_{i}}^{(i-1)}\right)\left\{M_{T_{i}}\left(\pi^{(i-1)}\right) \mathcal{G}_{T_{i}}\left(\pi^{(i-1)}\right)\right. \\
& \left.\quad \times\left[\dot{\tilde{\pi}}^{(i-1)}\left(\partial_{\pi} W\left(\pi^{(i-1)}\right)-\partial_{\pi} W\left(\pi^{(i-2)}\right)\right)(s)\right]\right\} \|_{L_{x}^{1}} d s \\
& \lesssim \int_{0}^{t-1}(t-s)^{-1-\epsilon}\left\|\left\langle x-y^{(i-1)}(s)\right\rangle^{\theta} \dot{\tilde{\pi}}^{(i-1)}\left(\partial_{\pi} W\left(\pi^{(i-1)}\right)-\partial_{\pi} W\left(\pi^{(i-2)}\right)\right)(s)\right\|_{L_{x}^{1}} d s \\
& \lesssim\left[\sup _{t \in\left[0, T_{i-1}\right]}\left|\tilde{\pi}^{(i-1)}-\tilde{\pi}^{(i-2)}\right|(t)\right] A C \delta \int_{0}^{t-1}(t-s)^{-1-\epsilon} s^{-1-\frac{\epsilon}{2}} d s \\
& \lesssim A C \delta \sup _{t \in\left[0, T_{i-1}\right]}\langle t\rangle^{1+\frac{\epsilon}{2}\left|\dot{\tilde{\pi}}^{(i-1)}-\dot{\tilde{\pi}}^{(i-2)}\right|(t) .}
\end{aligned}
$$

We have used Lemma 4.8 .

Next, we treat the difference $N\left(Z^{(i-1)}, \pi^{(i-1)}\right)-N\left(Z^{(i-2)}, \pi^{(i-2)}\right)$ in the nonlinearity. We split

$$
\begin{aligned}
N\left(Z^{(i-1)}, \pi^{(i-1)}\right)-N\left(Z^{(i-2)}, \pi^{(i-2)}\right)= & N\left(Z^{(i-1)}, \pi^{(i-1)}\right)-N\left(Z^{(i-2)}, \pi^{(i-1)}\right) \\
& +N\left(Z^{(i-2)}, \pi^{(i-1)}\right)-N\left(Z^{(i-2)}, \pi^{(i-2)}\right) .
\end{aligned}
$$

Proceeding as in the estimate of $\left\|U_{d i s}^{(i, i-1)}\right\|_{L_{x}^{2}}$, we write

$$
\begin{aligned}
& \int_{0}^{t-1}(t-s)^{-1-\epsilon} \|\langle x\rangle^{\theta} P_{s}\left(\alpha_{T_{i}}^{(i-1)}\right)\left\{M_{T_{i}}\left(\pi^{(i-1)}\right) \mathcal{G}_{T_{i}}\left(\pi^{(i-1)}\right)\right. \\
& \left.\quad \times\left[N\left(Z^{(i-1)}, \pi^{(i-1)}\right)-N\left(Z^{(i-2)}, \pi^{(i-1)}\right)\right](s)\right\} \|_{L_{x}^{1}} d s \\
& =\int_{0}^{1} \int_{0}^{t-1}(t-s)^{-1-\epsilon} \|\langle x\rangle^{\theta} P_{s}\left(\alpha_{T_{i}}^{(i-1)}\right)\left\{M _ { T _ { i } } ( \pi ^ { ( i - 1 ) } ) \mathcal { G } _ { T _ { i } } ( \pi ^ { ( i - 1 ) } ) \left[\left(Z^{(i-1)}-Z^{(i-2)}\right)\right.\right. \\
& \left.\left.\times \partial_{Z} N\left(Z^{(i-2)}+\mu\left(Z^{(i-1)}-Z^{(i-2)}\right), \pi^{(i-1)}\right)\right](s)\right] \|_{L_{x}^{1}} d s d \mu .
\end{aligned}
$$

We use Lemma 2.7 to reduce this to two estimates, a local and nonlocal one. First, we get (by moving the multiplier $\langle x\rangle^{\theta}$ inside and then removing $P_{s}\left(\alpha_{T_{i}}^{(i-1)}\right)$, etc.)

$$
\begin{aligned}
& \int_{0}^{t-1}(t-s)^{-1-\epsilon} \|\left\langle x-y^{(i-1)}(s)\right\rangle^{\theta}\left[Z^{(i-1)}-Z^{(i-2)}\right](s) \\
& \times\left|Z^{(i-2)}+\mu\left(Z^{(i-1)}-Z^{(i-2)}\right)\right|(s) \phi\left(.-y^{(i-1)}(s)\right) \|_{L_{x}^{1}} d s \\
& \lesssim A^{2} C \delta \sup _{t \in\left[0, T_{i-1}\right]}\left\|\left\langle x-y^{(i-2)}(t)\right\rangle^{-\theta}\left(Z^{(i-1)}-Z^{(i-2)}\right)(t)\right\|_{L_{x}^{\infty}} \int_{0}^{t-1}(t-s)^{-1-\epsilon}\langle s\rangle^{-1-\epsilon} d s .
\end{aligned}
$$


For the nonlocal term, we estimate if we assume (as we may) that $0<\epsilon<\sigma-1-2 \theta$

$$
\begin{aligned}
& \int_{0}^{t-1}(t-s)^{-1-\epsilon} \|\left\langle x-y^{(i-1)}(s)\right\rangle^{\theta}\left[Z^{(i-1)}-Z^{(i-2)}\right](s) \\
& \times\left|Z^{(i-2)}+\mu\left(Z^{(i-1)}-Z^{(i-2)}\right)\right|^{2 \sigma}(s) \|_{L_{x}^{1}} d s \\
& \lesssim A^{\theta}\left[\sup _{t \in\left[0, T_{i-1}\right]}\left\|\left\langle x-y^{(i-2)}(t)\right\rangle^{-\theta}\left(Z^{(i-1)}-Z^{(i-2)}\right)(t)\right\|_{L_{x}^{\infty}}\right] \\
& \times \int_{0}^{t-1}(t-s)^{-1-\epsilon}\left\|\left\langle x-y^{i-1}(s)\right\rangle^{2 \theta}\left|Z^{(i-2)}+\mu\left(Z^{(i-1)}-Z^{(i-2)}\right)\right|^{2 \sigma}(s)\right\|_{L_{x}^{1}} d s \\
& \lesssim A^{2}(C \delta)^{4}\left[\sup _{t \in\left[0, T_{i-1}\right]}\left\|\left\langle x-y^{(i-2)}(t)\right\rangle^{-\theta}\left(Z^{(i-1)}-Z^{(i-2)}\right)(t)\right\|_{\left.L_{x}^{\infty}\right]} .\right.
\end{aligned}
$$

We have used Lemma 6.12 Furthermore, using Lemma 4.8 as well as Lemma 2.7. we get

$$
\begin{aligned}
& \left|N\left(Z^{(i-2)}, \pi^{(i-1)}\right)-N\left(Z^{(i-2)}, \pi^{(i-2)}\right)\right|(s) \\
& \lesssim \sup _{0 \leq \mu \leq 1}\langle s\rangle\left|\tilde{\pi}^{(i-1)}-\tilde{\pi}^{(i-2)}\right|(s)\left|Z^{(i-2)}\right|^{2}(s) \phi\left(.-\mu y^{(i-2)}(s)-(1-\mu) y^{(i-1)}(s)\right) \\
& \quad+\left|Z^{(i-2)}\right|(s)^{2 \sigma+1} .
\end{aligned}
$$

Plugging this back in, we get for the contribution of the local term

$$
\begin{aligned}
& \int_{0}^{t-1}(t-s)^{-1-\epsilon}\langle s\rangle \sup _{0 \leq \mu \leq 1} \|\left\langle x-y^{(i-1)}(s)\right\rangle^{\theta}\left|\tilde{\pi}^{(i-1)}-\tilde{\pi}^{(i-2)}\right|(s) \\
& \quad \times\left|Z^{(i-2)}\right|^{2}(s) \phi\left(.-\mu y^{(i-2)}(s)\right) \|_{L_{x}^{1}} d s \\
& \lesssim A^{2} C^{2} \delta\left[\sup _{s \in\left[0, T_{i-1}\right]}\langle s\rangle^{1+\frac{\epsilon}{2}}\left|\dot{\tilde{\pi}}^{(i-1)}-\dot{\tilde{\pi}}^{(i-2)}\right|(s)\right] \int_{0}^{t-1}(t-s)^{-1-\epsilon}\langle s\rangle^{-1-\epsilon} d s,
\end{aligned}
$$

while for the contribution of the nonlocal term, we get

$$
\begin{aligned}
& \int_{0}^{t-1}(t-s)^{-1-\epsilon}\langle s\rangle \sup _{0 \leq \mu \leq 1}\left\|\left\langle x-y^{(i-1)}(s)\right\rangle^{\theta}\left|\tilde{\pi}^{(i-1)}-\tilde{\pi}^{(i-2)}\right|(s)\left|Z^{(i-2)}\right|(s)^{2 \sigma+1}\right\|_{L_{x}^{1}} d s \\
& \lesssim A(C \delta)^{2 \sigma}\left[\sup _{s \in\left[0, T_{i-1}\right]}\langle s\rangle^{1+\frac{\epsilon}{2}}\left|\dot{\tilde{\pi}}^{(i-1)}-\dot{\tilde{\pi}}^{(i-2)}\right|(s)\right] \int_{0}^{t-1}(t-s)^{-1-\epsilon}\langle s\rangle^{\frac{3}{2}+\theta-\sigma} d s .
\end{aligned}
$$

Finally, we need to control the following two local terms:

$$
\begin{aligned}
& \int_{0}^{t-1}(t-s)^{-1-\epsilon}\left\|\left\langle x-y^{(i-1)}(s)\right\rangle^{\theta}\left[\mathcal{H}\left(\pi^{(i-2)}\right)-\mathcal{H}\left(\pi^{(i-1)}\right)\right](s) Z^{(i-1)}\right\|_{L_{x}^{1}} d s \\
& \lesssim \sup _{s \in\left[0, T_{i-1}\right]}\langle s\rangle^{1+\frac{\epsilon}{2}}\left|\dot{\tilde{\pi}}^{(i-1)}-\dot{\tilde{\pi}}^{(i-2)}\right|(s) A^{2} C \delta \int_{0}^{t-1}(t-s)^{-1-\epsilon} s^{-\frac{\epsilon}{2}} d s
\end{aligned}
$$

where we have used Lemma 4.8, Similarly, by Lemma 2.4 ,

$$
\begin{aligned}
& \int_{0}^{t-1}(t-s)^{-1-\epsilon}\left\|\left\langle x-y^{(i-1)}(s)\right\rangle^{\theta}\left[\mathcal{H}\left(\pi^{(i-1)}\right)(t)-\mathcal{H}\left(\pi_{T_{i}}^{(i-1)}(t)\right)\right]\left(Z^{(i)}-Z^{(i-1)}\right)\right\|_{L_{x}^{1}} d s \\
& \lesssim C \delta\left[\sup _{s \in\left[0, T_{i-1}\right]}\left\|\left\langle x-y^{(i-2)}(s)\right\rangle^{-\theta}\left(Z^{(i)}-Z^{(i-1)}\right)\right\|_{L_{x}^{\infty}}\right] \int_{0}^{t-1}(t-s)^{-1-\epsilon} s^{-\epsilon} d s .
\end{aligned}
$$


This completes estimating the first integral expression in (4.20). The second is handled similarly and is hence omitted. We still need to estimate the contribution from the free term. The fact that the difference $\left(Z^{(i)}-Z^{(i-1)}\right)(0)$ is a local term ensures that this can be accomplished exactly along the same lines as for $\left\|\left(Z^{(i)}-Z^{(i-1)}\right)(0)\right\|_{L_{x}^{2}}$, which we dealt with earlier. This completes part (D).

(E): The estimate for $\left\|\langle x\rangle^{-\theta} U_{h y p}^{(i, i-1)}\right\|_{L_{x}^{\infty}}$. This is handled exactly like the corresponding contribution of $\left\|\langle t\rangle^{-1} U_{h y p}^{(i, i-1)}\right\|_{L_{x}^{2}}$, on account of the fact that this term is local.

(F): The estimate for $\left\|\langle x\rangle^{-\theta} U_{\text {root }}^{(i, i-1)}\right\|_{L_{x}^{\infty}}$. Use again that

$$
\begin{aligned}
\left\langle U_{\text {root }}^{(i, i-1)}, \tilde{\xi}_{\ell}\left(\pi^{(i-1)}\right)\right\rangle & =-\left\langle U_{\text {dis }}^{(i, i-1)}, \tilde{\xi}_{\ell}\left(\pi^{(i-1)}\right)\right\rangle-\left\langle U_{\text {hyp }}^{(i, i-1)}, \tilde{\xi}_{\ell}\left(\pi^{(i-1)}\right)\right\rangle \\
& +\left\langle Z^{(i-1)}, \xi_{\ell}\left(\pi^{(i-2)}\right)-\xi_{\ell}\left(\pi^{(i-1)}\right)\right\rangle .
\end{aligned}
$$

One combines the previous estimates for $\left\|\langle x\rangle^{-\theta} U_{d i s}^{(i, i-1)}\right\|_{L_{x}^{\infty}},\left\|\langle x\rangle^{-\theta} U_{h y p}^{(i, i-1)}\right\|_{L_{x}^{\infty}}$ with the following:

$$
\left|\left\langle Z^{(i-1)}, \xi_{\ell}\left(\pi^{(i-2)}\right)-\xi_{\ell}\left(\pi^{(i-1)}\right)\right\rangle\right|(t) \lesssim A C \delta \sup _{s \in\left[0, T_{i-1}\right]}\langle s\rangle^{1+\frac{\epsilon}{2}}\left|\dot{\tilde{\pi}}^{(i-1)}-\dot{\tilde{\pi}}^{(i-2)}\right|(s) .
$$

(G): Estimating $\sup _{t \in\left[T_{i-1}, T_{i}\right]}\left\|\langle x\rangle^{-\theta} U^{(i, i-1)}(t)\right\|_{L_{x}^{\infty}}$. This is again accomplished by using the a priori estimates. We get for $t \in\left[T_{i-1}, T_{i}\right]$

$$
\left\|\langle x\rangle^{-\theta} U^{(i, i-1)}(t)\right\|_{L_{x}^{\infty}} \lesssim(A+1) C \delta T_{i}^{-1-\epsilon},
$$

taking into account the translation effect of

$$
M_{T_{i-1}}\left(\pi^{(i-1)}\right)(t) \mathcal{G}_{T_{i-1}}\left(\pi^{(i-1)}\right)(t) \mathcal{G}_{T_{i-2}}\left(\pi^{(i-2)}\right)(t)^{-1} M_{T_{i-2}}\left(\pi^{(i-2)}\right)(t)^{-1} .
$$

Putting these estimates together completes the estimation for the norms involving $Z^{(i)}-Z^{(i-1)}$.

(H): We proceed to estimate $\sup _{t \in\left[0, T_{i}\right]}\langle t\rangle^{1+\frac{\epsilon}{2}}\left|\dot{\tilde{\pi}}^{(i)}-\dot{\tilde{\pi}}^{(i-1)}\right|(t)$. First, consider the case $t \in\left[0, T_{i-1}\right]$. Use the equation

$$
\begin{aligned}
& -i\left[\left\langle\dot{\pi}^{(i)} \partial_{\pi} W\left(\pi^{(i-1)}\right), \xi_{l}\left(\pi^{(i-1)}\right)\right\rangle-\left\langle\dot{\tilde{\pi}}^{(i-1)}, \partial_{\pi} W\left(\pi^{(i-2)}\right), \xi_{l}\left(\pi^{(i-2)}\right)\right\rangle\right] \\
& =\left\langle Z^{(i)}(t), i \dot{\tilde{\pi}}^{(i-1)} \tilde{\mathcal{S}}_{\ell}\left(\pi^{(i-1)}\right)(t)\right\rangle-\left\langle N\left(Z^{(i-1)}, \pi^{(i-1)}\right), \xi_{\ell}\left(\pi^{(i-1)}\right)(t)\right\rangle \\
& -\left(\left\langle Z^{(i-1)}(t), i \dot{\tilde{\pi}}^{(i-2)} \tilde{\mathcal{S}}_{\ell}\left(\pi^{(i-2)}\right)(t)\right\rangle-\left\langle N\left(Z^{(i-2)}, \pi^{(i-2)}\right), \xi_{\ell}\left(\pi^{(i-2)}\right)(t)\right\rangle\right) .
\end{aligned}
$$

Note that

$$
\begin{aligned}
\left|\dot{\tilde{\pi}}^{(i)}-\dot{\tilde{\pi}}^{(i-1)}\right|(t) \lesssim & \left|\left\langle\dot{\pi}^{(i)} \partial_{\pi} W\left(\pi^{(i-1)}\right), \xi_{l}\left(\pi^{(i-1)}\right)\right\rangle-\left\langle\dot{\pi}^{(i-1)} \partial_{\pi} W\left(\pi^{(i-2)}\right), \xi_{l}\left(\pi^{(i-2)}\right)\right\rangle\right| \\
& +\langle t\rangle^{1+\frac{\epsilon}{2}} \dot{\tilde{\pi}}^{(i)}(t)\left|\tilde{\pi}^{i-1}(t)-\tilde{\pi}^{(i-2)}(t)\right| .
\end{aligned}
$$

First we have

$$
\begin{aligned}
\left|\left\langle Z^{(i)}-Z^{(i-1)}, i \dot{\tilde{\pi}}^{(i-1)} \mathcal{S}_{\ell}\left(\pi^{(i-1)}\right)\right\rangle(t)\right| \\
\quad \lesssim C \delta\langle t\rangle^{-1-\epsilon} \sup _{t \in\left[0, T_{i-1}\right]}\langle t\rangle^{-1}\left\|\left(Z^{(i)}-Z^{(i-1)}\right)(t)\right\|_{L_{x}^{2}}
\end{aligned}
$$

Of course this term will then be moved to the left. Next, we have

$$
\begin{aligned}
& \left|\left\langle Z^{(i-1)},\left[\dot{\tilde{\pi}}^{(i-1)}-\dot{\tilde{\pi}}^{(i-2)}\right] \mathcal{S}_{\ell}\left(\pi^{(i-2)}\right)\right\rangle(t)\right| \\
& \lesssim\langle t\rangle^{-1-\frac{\epsilon}{2}}\left\|Z^{(i-1)}\right\|_{L_{x}^{2}} \sup _{s \in\left[0, T_{i-1}\right]}\langle s\rangle^{1+\frac{\epsilon}{2}}\left|\dot{\tilde{\pi}}^{(i-1)}-\dot{\tilde{\pi}}^{(i-2)}\right|(s) .
\end{aligned}
$$


Furthermore, using a variant of Lemma 4.8, we get

$$
\begin{aligned}
& \left|\left\langle Z^{(i-1)}, \dot{\tilde{\pi}}^{(i-1)}\left(\mathcal{S}_{\ell}\left(\pi^{(i-1)}\right)-\mathcal{S}_{\ell}\left(\pi^{(i-2)}\right)\right)(t)\right\rangle\right| \\
& \lesssim\left\|Z^{(i-1)}\right\|_{L_{x}^{2}}\langle t\rangle^{1+\frac{\epsilon}{2}}\left|\dot{\tilde{\pi}}^{(i-1)}(t) \|\left(\pi^{(i-1)}-\pi^{(i-2)}\right)(t)\right|,
\end{aligned}
$$

which is bounded by (always keeping in mind that $t \in\left[0, T_{i-1}\right]$ )

$$
C \delta\langle t\rangle^{-1-\frac{\epsilon}{2}} \sup _{s \in\left[0, T_{i-1}\right]}\langle s\rangle^{1+\frac{\epsilon}{2}}\left|\dot{\tilde{\pi}}^{(i-1)}-\dot{\tilde{\pi}}^{(i-2)}\right|(s) .
$$

The difference

$$
\left\langle N\left(Z^{(i-1)}, \pi^{(i-1)}\right), \tilde{\xi}_{\ell}\left(\pi^{(i-1)}\right)(t)\right\rangle-\left\langle N\left(Z^{(i-2)}, \pi^{(i-2)}\right), \tilde{\xi}_{\ell}\left(\pi^{(i-2)}\right)(t)\right\rangle
$$

is handled as before. Indeed, one obtains

$$
\begin{gathered}
\left|\left\langle N\left(Z^{(i-1)}, \pi^{(i-1)}\right), \tilde{\xi}_{\ell}\left(\pi^{(i-1)}\right)(t)\right\rangle-\left\langle N\left(Z^{(i-1)}, \pi^{(i-2)}\right), \tilde{\xi}_{\ell}\left(\pi^{(i-1)}\right)(t)\right\rangle\right| \\
\lesssim\langle t\rangle^{1+\frac{\epsilon}{2}}\left|\tilde{\pi}^{(i-1)}-\tilde{\pi}^{(i-2)}\right|(t)\left|\partial_{\pi} N\left(Z^{(i-1)}, \mu \pi^{(i-1)}+(1-\mu) \pi^{(i-2)}(t)\right)\right| \\
\lesssim A^{2}\left[C^{2} \delta^{2}+(C \delta)^{2 \sigma}\right]\langle t\rangle^{-1-\frac{\epsilon}{2}} \sup _{t \in\left[0, T_{i-1}\right]}\langle t\rangle^{1+\frac{\epsilon}{2}}\left|\left(\dot{\tilde{\pi}}^{(i-1)}-\dot{\tilde{\pi}}^{(i-1)}\right)(t)\right| \\
\left|\left\langle N\left(Z^{(i-1)}, \pi^{(i-2)}\right), \tilde{\xi}_{\ell}\left(\pi^{(i-1)}\right)(t)\right\rangle-\left\langle N\left(Z^{(i-2)}, \pi^{(i-2)}\right), \tilde{\xi}_{\ell}\left(\pi^{(i-1)}\right)(t)\right\rangle\right| \\
\lesssim\left|Z^{(i-1)}-Z^{(i-2)}\right|(t)\left|\partial_{Z} N\left(\mu Z^{(i-1)}+(1-\mu) Z^{(i-2)}, \pi^{(i-2)}\right)(t)\right| \\
\lesssim A^{2}\left[C \delta+(C \delta)^{2 \sigma}\right]\langle t\rangle^{-1-\epsilon} \sup _{s \in\left[0, T_{i-1}\right]}\left\|\left\langle x-y^{(i-1)}(s)\right\rangle^{-\theta}\left(Z^{(i-1)}-Z^{(i-2)}\right)\right\|_{L_{x}^{\infty} .}
\end{gathered}
$$

(I): Finally, consider the case $t \in\left[T_{i-1}, T_{i}\right]$. Then we estimate

$$
\begin{aligned}
& \mid\left\langle Z^{(i)}(t), i \dot{\tilde{\pi}}^{(i-1)} \tilde{\mathcal{S}}_{\ell}\left(\pi^{(i-1)}\right)(t)\right\rangle-\left\langle N\left(Z^{(i-1)}, \pi^{(i-1)}\right), \tilde{\xi}_{\ell}\left(\pi^{(i-1)}\right)(t)\right\rangle \\
& \quad-\left(\left\langle Z^{(i-1)}(t), i \dot{\tilde{\pi}}^{(i-2)} \tilde{\mathcal{S}}_{\ell}\left(\pi^{(i-2)}\right)(t)\right\rangle-\left\langle N\left(Z^{(i-2)}, \pi^{(i-2)}\right), \tilde{\xi}_{\ell}\left(\pi^{(i-2)}\right)(t)\right\rangle\right) \mid \\
& \lesssim\left[(A+1)^{2}(C \delta)^{2}+(C \delta)^{2 \sigma}\right] T_{i}^{-2-\epsilon} .
\end{aligned}
$$

Putting all of the preceding estimates (A)-(I) together, we obtain the claim of Proposition 4.5 .

We have now shown that Definition 4.3 results in iterates satisfying the a priori estimates (4.3). We now need to show that the $\left(\pi^{(i)}, Z^{(i)}\right)$ converge in a suitable sense. We have the following theorem.

Theorem 4.9. For $i \geq 1, j \geq i$, the following inequality hold 15 :

$$
\left\|\left(\tilde{\pi}^{(i)}-\tilde{\pi}^{(j)}, Z^{(i)}-Z^{(j)}\right)\right\|_{Y^{(i)}\left(\left[0, T_{i}\right]\right)} \lesssim i^{-1} .
$$

There exists $(\pi, Z) \in \tilde{X}_{*}(\pi)$ solving (2.1) in the $H^{1}$-sense in addition to satisfying the orthogonality relations

$$
\left\langle Z, \xi_{\ell}(\pi(t))\right\rangle=0 \quad \forall t \in[0, \infty)
$$

with the following property: for every $T>0$, we have

$$
\lim _{i \rightarrow \infty}\left\|\left(\pi-\pi^{(i)}, Z-Z^{(i)}\right)\right\|_{\tilde{X}_{*}(\pi)([0, T])} \rightarrow 0 .
$$

In particular, we get

$$
\|(\pi, Z)\|_{\tilde{X}_{*}(\pi)} \leq C \delta
$$

\footnotetext{
${ }^{15}$ This convergence is rather slow, of course, and can be significantly improved by choosing $T_{i}$ less conservatively.
} 
Also, we have

$$
Z(0)=\left(\frac{R_{0}}{R_{0}}\right)+h f^{+}\left(\alpha_{\infty}\right)+\sum_{j=1}^{4} a_{j} \tilde{\eta}_{j}\left(\alpha_{\infty}\right)
$$

for suitable $h, a_{j}(h) \in \mathbb{R}$, where $h=h\left(R_{0}\right)$ depends in a Lipschitz continuous fashion on $R_{0}$. Finally, $(\pi, Z)$ is the unique solution with these initial data and satisfying the above bounds and orthogonality relations.

Proof. We need to show that the $\left(\pi^{(i)}, Z^{(i)}\right)$ form a Cauchy sequence in a suitable sense. This follows from the following pair of inequalities. Let $j \geq i$.

$$
\begin{aligned}
& \left\|\left(\tilde{\pi}^{(i)}-\tilde{\pi}^{(j)}, Z^{(i)}-Z^{(j)}\right)\right\|_{Y^{(i)}\left(\left[0, T_{i}\right]\right)} \\
& \lesssim\left[A^{2} C \delta+A^{2}(C \delta)^{2 \sigma}\right]\left\|\left(\tilde{\pi}^{(i-1)}-\tilde{\pi}^{(j-1)}, Z^{(i-1)}-Z^{(j-1)}\right)\right\|_{Y^{(i-1)}\left(\left[0, T_{i-1}\right]\right)} \\
& +\left[1+(A+1)^{2}(C \delta)^{2}+(C \delta)^{2 \sigma}\right] T_{i}^{-1}, \\
& T_{i} \sup _{j \geq i} \sup _{t \in\left[0, T_{i}\right]}\left|\tilde{\pi}^{(i)}-\tilde{\pi}^{(j)}\right|(t) \lesssim A .
\end{aligned}
$$

The proof of these follows along the exact same lines as the proof of Proposition 4.5, One uses the fact that on account of the a priori estimates already established, we have $(j \geq 0)$

$$
T_{0} \sup _{j \geq i} \sup _{t \in\left[0, T_{0}\right]}\left|\pi^{(0)}-\pi^{(j)}\right|(t) \lesssim A .
$$

If one iterates the first of the above two inequalities, one obtains

$$
\left\|\left(\tilde{\pi}^{(i)}-\tilde{\pi}^{(j)}, Z^{(i)}-Z^{(j)}\right)\right\|_{Y^{(i)}\left(\left[0, T_{i}\right]\right)} \lesssim i^{-1} .
$$

Now choose $T>0$ and $i_{0}$ such that $T_{i_{0}}>T$. Then we see that for $i \geq i_{0}, j \geq i$, we have

$$
\left\|\left(\tilde{\pi}^{(i)}-\tilde{\pi}^{(j)}, Z^{(i)}-Z^{(j)}\right)\right\|_{Y^{(i)}([0, T])} \lesssim i^{-1} .
$$

In particular, the numbers $\langle t\rangle^{1+\frac{\epsilon}{2}} \dot{\tilde{\pi}}^{(i)}(t)$ converge uniformly on $[0, T]$, whence also $\left.\pi^{(i)}\right|_{[0, T]}$ converge toward some Lipschitz continuous path $\pi_{T}$ on $[0, T]$. Actually, this path is $C^{1}$, since $\dot{\pi}$ is locally the uniform limit of continuous functions. Note that if we define $y_{T}(t)=\int_{0}^{t} v_{T}(s) d s+D_{T}(s)$, we have

$$
\left.y^{(i)}\right|_{[0, T]}(t) \rightarrow y_{T}(t),
$$

and consequently we have

$$
\|\cdot\|_{Y^{(i)}[0, T]} \rightarrow\|\cdot\|_{Y[0, T]},
$$

where $\|\cdot\|_{Y[0, T]}$ is defined like $\|\cdot\|_{Y^{(i)}[0, T]}$ with $y^{(i-1)}(t)$ replaced by $y(t)$. In summary, we get

$$
\lim _{i, j \rightarrow \infty}\left\|\left(\tilde{\pi}^{(i)}-\tilde{\pi}^{(j)}, Z^{(i)}-Z^{(j)}\right)\right\|_{Y[0, T]}=0 .
$$

This in conjunction to the a priori estimates implies that the $\left.Z^{(i)}\right|_{[0, T]}$ converge pointwise toward some function $Z_{T} \in X_{*}([0, T])$, which satisfies

$$
\lim _{i \rightarrow \infty}\left\|\left(\pi_{T}-\pi^{(i)}, Z_{T}-\left.Z^{(i)}\right|_{[0, T]}\right)\right\|_{X_{*}[0, T]}=0 .
$$

Clearly $\left(\pi_{T}, Z_{T}\right)$ weakly solves (2.1) and satisfies the orthogonality relations on $[0, T]$. Indeed, we can improve this statement by observing that the norm $\|\cdot\|_{Y^{(i)}\left(\left[0, T_{i}\right]\right)}$ in Proposition 4.5 may be strengthened to also include

$$
\sup _{t \in\left[0, T_{i}\right]}\left[\langle t\rangle^{-1}\|Z(t)\|_{H^{1}}+\left\|\left\langle x-y^{(i-1)}\right\rangle^{-\frac{1}{2}-2 \epsilon} \partial_{x} Z(t)\right\|_{L_{x}^{q}}\right], \quad q \text { as in }\|\cdot\|_{X_{*}} .
$$


The justification for this is as in the proof of Proposition 4.4. This in particular entails that

$$
Z_{T} \in C\left([0, T], H^{1}(\mathbb{R}) \times H^{1}(\mathbb{R})\right) \cap C^{1}\left([0, T), H^{-1}(\mathbb{R}) \times H^{-1}(\mathbb{R})\right),
$$

and $Z$ solves the equation in the $H^{1}$-sense. Replacing $T$ by a larger $\tilde{T}$, we can compatibly extend $\left(\pi_{T}, Z_{T}\right)$ to a larger time interval, whence all the way to $[0, \infty)$. Then the a priori estimates imply that the $(\pi, Z)$ thus constructed lies in $X_{*}$, as well as

$$
C\left([0, \infty), H^{1}(\mathbb{R}) \times H^{1}(\mathbb{R})\right) \cap C^{1}\left([0, \infty), H^{-1}(\mathbb{R}) \times H^{-1}(\mathbb{R})\right) .
$$

In particular, the limit $\alpha_{\infty}:=\lim _{t \rightarrow \infty} \alpha(t)$ exists. The estimate (4.21) implies that

$$
Z^{(i)}(0) \rightarrow Z(0)
$$

in the $L^{2}$-sense. In particular, recalling

$$
Z^{(i)}(0)=\left(\begin{array}{c}
R_{0} \\
\bar{R}_{0}
\end{array}\right)+h^{(i-1)} f^{+}\left(\alpha_{T_{i}}^{(i-1)}\right)+\sum_{k=1}^{4} a_{k}^{(i-1)} \tilde{\eta}_{k}\left(\alpha_{T_{i}}^{(i-1)}\right),
$$

we get $h^{(i-1)} \rightarrow h^{+}$for suitable $h^{+} \in \mathbb{R}$, and similarly $a_{k}^{(i-1)} \rightarrow a_{k}$ for suitable $a_{k} \in \mathbb{R}$. Moreover, on account of the a priori estimates, we have

$$
\left|\alpha_{T_{i}}^{(i-1)}-\alpha^{(i-1)}(T)\right|<T^{-1-\epsilon},\left|\alpha_{\infty}-\alpha(T)\right|<T^{-1-\epsilon}
$$

for $i$ suitably large. Hence

$$
\left|\alpha_{T_{i}}^{(i-1)}-\alpha_{\infty}\right|<2 T^{-1-\epsilon}+\left|\alpha(T)-\alpha^{(i-1)}(T)\right|
$$

and therefore

$$
\lim _{i \rightarrow \infty}\left|\alpha_{T_{i}}^{(i-1)}-\alpha_{\infty}\right|<2 T^{-1-\epsilon} .
$$

Letting $T \rightarrow \infty$, we get $\lim _{i \rightarrow \infty} \alpha_{T_{i}}^{(i-1)}=\alpha_{\infty}$, whence indeed

$$
Z(0)=\left(\begin{array}{c}
R_{0} \\
\bar{R}_{0}
\end{array}\right)+h f^{+}\left(\alpha_{\infty}\right)+\sum_{k=1}^{4} a_{k} \tilde{\eta}_{k}\left(\alpha_{\infty}\right) .
$$

We now verify that $h^{+}, a_{k}$ depend in a Lipschitz continuous manner on $R_{0}$. We claim the following:

Lemma 4.10. Let $(\pi, Z)$ be the solution associated with $\left(\frac{R_{0}}{R_{0}}\right)$, and let $\left(\pi^{*}, Z^{*}\right)$ be the solution associated with $\left(\frac{R_{0}^{*}}{R_{0}^{*}}\right)$. Then the following inequality hold 16 :

$$
\left\|\langle t\rangle^{-1}\left[Z-Z^{*}\right]\right\|_{L_{t}^{\infty} L_{x}^{2}\left(\left[0,\left\|R_{0}-R_{0}^{*}\right\|^{-1}\right]\right)}+\left\|\left(\tilde{\pi}-\tilde{\pi^{*}}\right)(t)\right\|_{L_{t}^{\infty}\left(\left[0,\left\|R_{0}-R_{0}^{*}\right\|^{-1}\right]\right)} \lesssim\left\|R_{0}-R_{0}^{*}\right\| .
$$

Assuming this lemma for now, we introduce $U(t)=M_{\infty}(\pi)(t) \mathcal{G}_{\infty}(\pi)(t) Z(t)$, and analogously for $U^{*}$. The preceding considerations imply that we may write

$$
U=U_{\text {dis }}+U_{\text {root }}+U_{\text {hyp }} \text { with respect to } \mathcal{H}\left(\alpha_{\infty}\right),
$$

and we can write

$$
U_{\text {hyp }}=b^{+}(t) f^{+}\left(\alpha_{\infty}\right)+b^{-}(t) f^{-}\left(\alpha_{\infty}\right)
$$

\footnotetext{
${ }^{16}$ We shall assume in the following that $\left\|R_{0}-R_{0}^{*}\right\|<1$.
} 
where

$$
b^{+}(0)=-\int_{0}^{\infty} e^{-\gamma\left(\alpha_{\infty}\right) s} g^{+}(U, \pi)(s) d s
$$

and we have

$$
\begin{aligned}
& g^{+}(U, \pi)=P_{I m}^{+}\left(\alpha_{\infty}\right)\left[-i \dot{\tilde{\pi}} \partial_{\pi} \tilde{W}_{\infty}(\pi)+N(U, \pi)+V U\right] \\
& V=V(t) \\
& :=\left(\begin{array}{cc}
(\sigma+1)\left(\phi_{\infty}^{2 \sigma}(x)-\phi^{2 \sigma}\left(x+y_{\infty}-y\right)\right) & \sigma\left(\phi_{\infty}^{2 \sigma}(x)-e^{2 i \rho_{\infty}} \phi^{2 \sigma}\left(x+y_{\infty}-y\right)\right) \\
-\sigma\left(\phi_{\infty}^{2 \sigma}(x)-e^{-2 i \rho_{\infty}} \phi^{2 \sigma}\left(x+y_{\infty}-y\right)\right) & -(\sigma+1)\left(\phi_{\infty}^{2 \sigma}(x)-\phi^{2 \sigma}\left(x+y_{\infty}-y\right)\right)
\end{array}\right) \\
& i \dot{\pi} \partial_{\pi} \tilde{W}(\pi):=\dot{v}\left(\begin{array}{c}
-\left(x+y_{\infty}\right) e^{i \rho_{\infty}} \phi\left(x+y_{\infty}-y\right) \\
\left(x+y_{\infty}\right) e^{-i \rho_{\infty} \phi}\left(x+y_{\infty}-y\right)
\end{array}\right)+\dot{\gamma}\left(\begin{array}{c}
-e^{i \rho_{\infty} \phi}\left(x+y_{\infty}-y\right) \\
e^{-i \rho_{\infty} \phi}\left(x+y_{\infty}-y\right)
\end{array}\right) \\
& +i \dot{\alpha}\left(\begin{array}{c}
e^{i \rho_{\infty}} \partial_{\alpha} \phi\left(x+y_{\infty}-y\right) \\
e^{-i \rho_{\infty}} \partial_{\alpha} \phi\left(x+y_{\infty}-y\right)
\end{array}\right)+i \dot{D}\left(\begin{array}{c}
-e^{i \rho_{\infty}} \partial_{x} \phi\left(x+y_{\infty}-y\right) \\
-e^{-i \rho_{\infty}} \partial_{x} \phi\left(x+y_{\infty}-y\right)
\end{array}\right)
\end{aligned}
$$

and $N(U, \pi)$ is defined as in (2.23) with $T$ replaced by $\infty$. Plugging in the above estimate, one easily ${ }^{17}$ obtains the bound

$$
\left|\int_{0}^{\infty} e^{-\gamma\left(\alpha_{\infty}\right) s} g^{+}(U, \pi)(s) d s-\int_{0}^{\infty} e^{-\gamma\left(\alpha_{\infty}^{*}\right) s} g^{+}\left(U^{*}, \pi^{*}\right)(s) d s\right| \lesssim \delta\left\|R_{0}-R_{0}^{*}\right\| .
$$

Now one uses that (with an analogous equation determining $b^{*+}(0)$ )

$$
b^{+}(0)=P_{I m}^{+}\left(\alpha_{\infty}\right) M_{\infty}(\pi)(0) \mathcal{G}_{\infty}(\pi)(0)\left[\left(\begin{array}{c}
R_{0} \\
\bar{R}_{0}
\end{array}\right)+h f^{+}\left(\alpha_{\infty}\right)+\sum_{k=1}^{4} a_{k} \tilde{\eta}_{k}\left(\alpha_{\infty}\right)\right]
$$

Observing that

$$
P_{I m}^{+}\left(\alpha_{\infty}\right)\left(\begin{array}{c}
R_{0} \\
\bar{R}_{0}
\end{array}\right)=\left[P_{I m}^{+}\left(\alpha_{\infty}\right)-P_{I m}^{+}\left(\alpha_{0}\right)\right]\left(\begin{array}{c}
R_{0} \\
\bar{R}_{0}
\end{array}\right)
$$

one infers from the preceding that

$$
\left|h-h^{*}\right| \lesssim \delta\left\|R_{0}-R_{0}^{*}\right\|
$$

Furthermore, exploiting the orthogonality relations determining $U_{\text {root }}, U_{\text {root }}^{*}$, one obtains a similar estimate for $a_{k}-a_{k}^{*}$ for all $k$. The argument just given also easily implies the bound

$$
|h|+\sum_{k=1}^{4}\left|a_{k}\right| \lesssim\left\|R_{0}\right\|^{2}
$$

We now turn to the proof of Lemma 4.10, which is based on a recursive inequality:

\footnotetext{
${ }^{17}$ Repeating estimates as in the proofs of Proposition 4.4 etc., and breaking the integrals into two parts, one over the interval $\left[0,\left\|R_{0}-R_{0}^{*}\right\|^{-1}\right]$ and the other over its complement, where the a priori estimates are used.
} 
Proof of Lemma 4.10. We recycle the notation from the proof of Proposition 4.4. etc. We claim that the following pair of inequalities hold true 18 for $j \geq i$ :

$$
\begin{aligned}
\|\left(\tilde{\pi}^{(i)}-\right. & \left.\tilde{\pi}^{*(i)}, Z^{(i)}-Z^{*(i)}\right) \|_{Y^{(i)}\left(\left[0, \min \left\{T_{i},\left\|R_{0}-R_{0}^{*}\right\|^{-1}\right\}\right]\right)} \\
\lesssim & {\left[A^{2} C \delta+A^{2}(C \delta)^{2 \sigma}\right] } \\
& \times\left\|\left(\tilde{\pi}^{(i-1)}-\tilde{\pi}^{*(i-1)}, Z^{(i-1)}-Z^{*(i-1)}\right)\right\|_{Y^{(i-1)}\left(\left[0, \min \left\{T_{i-1},\left\|R_{0}-R_{0}^{*}\right\|^{-1}\right\}\right]\right)} \\
& +\left[1+(A+1)^{2}(C \delta)^{2}+(C \delta)^{2 \sigma}\right] T_{i}^{-1}+\left\|R_{0}-R_{0}^{*}\right\|, \\
\min & \left\{T_{i},\left\|R_{0}-R_{0}^{*}\right\|^{-1}\right\} \sup _{t \in\left[0, \min \left\{T_{i},\left\|R_{0}-R_{0}^{*}\right\|^{-1}\right\}\right]}\left|\tilde{\pi}^{(i)}-\tilde{\pi}^{*(i)}\right|(t)<A .
\end{aligned}
$$

The proof of this proceeds inductively, assuming

$$
\sup _{0 \leq j \leq i-1} \min \left\{T_{j},\left\|R_{0}-R_{0}^{*}\right\|^{-1}\right\} \sup _{t \in\left[0, \min \left\{T_{j},\left\|R_{0}-R_{0}^{*}\right\|^{-1}\right\}\right]}\left|\tilde{\pi}^{(j)}-\tilde{\pi}^{*(j)}\right|(t)<A .
$$

Iterating as usual, it is then easy to see that one can retrieve the latter inequality for $j=i$ from the foregoing inequality. To prove the first inequality, one writes the difference equation satisfied by $Z-Z^{*}$ in the following fashion:

$$
\begin{aligned}
& i \partial_{t}\left(Z^{(i)}-Z^{*(i)}\right)-\mathcal{H}\left(\pi^{(i-1)}\right)\left(Z^{(i)}-Z^{*(i)}\right) \\
& =-\left[i \dot{\tilde{\pi}}^{(i)} \partial_{\pi} W\left(\pi^{(i-1)}\right)-i \dot{\tilde{\pi}}^{*(i)} \partial_{\pi} W\left(\pi^{*(i-1)}\right)\right] \\
& \quad+\left[N\left(Z^{(i-1)}, \pi^{(i-1)}\right)-N\left(Z^{*(i-1)}, \pi^{*(i-1)}\right)\right]+\left[\mathcal{H}\left(\pi^{*(i-1)}\right)-\mathcal{H}\left(\pi^{(i-1)}\right)\right] Z^{*(i)}, \\
& i\left[\dot{\tilde{\pi}}^{(i)}-\dot{\tilde{\pi}}^{*(i)}\right]=\left\langle Z^{(i)}(t), i \dot{\pi}^{(i-1)} \tilde{\mathcal{S}}_{\ell}\left(\pi^{(i-1)}\right)(t)\right\rangle-\left\langle N\left(Z^{(i-1)}, \pi^{(i-1)}\right), \tilde{\xi}_{\ell}\left(\pi^{(i-1)}\right)(t)\right\rangle \\
& -\left(\left\langle Z^{*(i)}(t), i \dot{\tilde{\pi}}^{*(i-1)} \tilde{\mathcal{S}}_{\ell}\left(\pi^{*(i-1)}\right)(t)\right\rangle-\left\langle N\left(Z^{*(i-1)}, \pi^{*(i-1)}\right), \tilde{\xi}_{\ell}\left(\pi^{*(i-1)}\right)(t)\right\rangle\right) .
\end{aligned}
$$

The estimation on the interval $\left[0, \min \left\{T_{i},\left\|R_{0}-R_{0}^{*}\right\|^{-1}\right\}\right]$ follows then almost verbatim the proof of Proposition 4.5. Note that the paths $\pi, \pi^{*}$ lead to compatible norms on this interval.

We have almost completed the proof of Theorem 4.9. All that is left is the uniqueness part. For this, consider the solutions $(\pi, Z) \in \tilde{X}_{*}(\pi),\left(\pi^{*}, Z^{*}\right) \in \tilde{X}_{*}\left(\pi^{*}\right)$ with identical initial data:

$$
\begin{aligned}
Z(0) & =\left(\begin{array}{c}
R_{0} \\
\bar{R}_{0}
\end{array}\right)+h f^{+}\left(\alpha_{\infty}\right)+\sum_{k=1}^{4} a_{k} \tilde{\eta}_{k}\left(\alpha_{\infty}\right) \\
& =Z^{*}(0)=\left(\begin{array}{c}
R_{0} \\
\bar{R}_{0}
\end{array}\right)+h^{*} f^{+}\left(\alpha_{\infty}^{*}\right)+\sum_{k=1}^{4} a_{k}^{*} \tilde{\eta}_{k}\left(\alpha_{\infty}^{*}\right) .
\end{aligned}
$$

We study the difference equation for $Z-Z^{*}, \pi-\pi^{*}$. On account of the a priori bounds, the two paths $y(t)=\int_{0}^{t} v(s) d s+D(t), y^{*}(t)=\int_{0}^{t} v^{*}(s) d s+D^{*}(t)$ differ by $A$ (say) on $[0, T]$ where $T=\delta^{-1}$. We estimate (the norm $\|\cdot\|_{Y}$ here is either with respect to $\pi$ or $\left.\pi^{*}\right)$

$$
\left\|\left(\tilde{\pi}-\tilde{\pi}^{*}, Z-Z^{*}\right)\right\|_{Y([0, T+10])} \lesssim \delta\left\|\left(\tilde{\pi}-\tilde{\pi}^{*}, Z-Z^{*}\right)\right\|_{Y([0, T])}+\delta T^{-1} .
$$

Implicit in the equation are the constants $A, C$ from before, which we assume to be chosen once and for all. From the above, we obtain in particular that

$$
\langle T+10\rangle \sup _{t \in[0, T+10]}\left|\tilde{\pi}-\tilde{\pi}^{*}\right|(t)<A .
$$

\footnotetext{
${ }^{18}$ Possibly shrinking $\delta$ and growing $A, C$ a bit.
} 
Now one repeats the same argument with $T+10$ instead $T$, etc. The conclusion is that

$$
\left\|\left(\tilde{\pi}-\tilde{\pi}^{*}, Z-Z^{*}\right)\right\|_{Y([0, T])}=0 \text { for all } T>0,
$$

whence the two solutions agree.

Proof of Theorem 1.1. In light of Theorem 4.9, we are almost done; we only need to verify the scattering statement. Given a solution $(\pi, Z)$ on $[0, \infty)$ constructed as in the preceding proof and with $Z(0)$ as given by (4.22), we define

$$
\Phi\left(R_{0}\right)=\text { upper entry of }\left[h f^{+}\left(\alpha_{\infty}\right)+\sum_{k=1}^{4} a_{k} \tilde{\eta}_{k}\left(\alpha_{\infty}\right)\right] \text {. }
$$

Define as usual $U(t)=M_{\infty}(\pi)(t) \mathcal{G}_{\infty}(\pi)(t) Z(t)$. We first seek a representation of the form

$$
U(t)=e^{-i t \mathcal{H}\left(\alpha_{\infty}\right)} U_{1}+o_{L^{2}}(1)
$$

for a suitable $U_{1} \in P_{s}\left(L^{2}(\mathbb{R})\right)$. Define

$$
U_{1}:=U_{d i s}(0)-i \int_{0}^{\infty} e^{i r \mathcal{H}\left(\alpha_{\infty}\right)} P_{s}\left(\alpha_{\infty}\right)[F(r)] d r
$$

where

$$
F(t):=i \partial_{t} U(t)-\mathcal{H}\left(\alpha_{\infty}\right) U(t)
$$

Clearly, we then have

$$
U(t)-e^{-i t \mathcal{H}\left(\alpha_{\infty}\right)} U_{1}=U_{\text {root }}+U_{\text {hyp }}-i \int_{t}^{\infty} e^{i r \mathcal{H}\left(\alpha_{\infty}\right)} P_{s}\left(\alpha_{\infty}\right)[F(r)] d r .
$$

On the other hand, the preceding estimates as well as the fact that both $U_{\text {root }}, U_{\text {hyp }}$ are local and satisfy suitable $L^{\infty}$ decay estimates imply that

$$
U_{\text {root }}+U_{\text {hyp }}-i \int_{t}^{\infty} e^{i r \mathcal{H}\left(\alpha_{\infty}\right)} P_{s}\left(\alpha_{\infty}\right)[F(r)] d r=o_{L^{2}}(1) .
$$

It remains to show that one has scattering for the evolution of $\mathcal{H}\left(\alpha_{\infty}\right)$. This is a standard Cook method argument. Indeed, write

$$
\begin{aligned}
\mathcal{H}\left(\alpha_{\infty}\right) & =\left(\begin{array}{cc}
-\partial_{x}^{2}+\alpha_{\infty}^{2} & 0 \\
0 & \partial_{x}^{2}-\alpha_{\infty}^{2}
\end{array}\right)+\left(\begin{array}{cc}
-(\sigma+1) \phi_{\infty}^{2 \sigma} & -\sigma \phi_{\infty}^{2 \sigma} \\
\sigma \phi_{\infty}^{2 \sigma} & (\sigma+1) \phi_{\infty}^{2 \sigma}
\end{array}\right) \\
& =: \mathcal{H}_{0}\left(\alpha_{\infty}\right)+V,
\end{aligned}
$$

where $\phi_{\infty}:=\phi\left(\cdot, \alpha_{\infty}\right)$. Then

$$
e^{-i t \mathcal{H}\left(\alpha_{\infty}\right)} U_{1}=e^{-i t \mathcal{H}_{0}\left(\alpha_{\infty}\right)} U_{1}-i \int_{0}^{t} e^{-i(t-s) \mathcal{H}_{0}\left(\alpha_{\infty}\right)} V e^{-i s \mathcal{H}\left(\alpha_{\infty}\right)} U_{1} d s
$$

and thus

$$
U_{2}:=\lim _{t \rightarrow \infty} e^{i t \mathcal{H}_{0}\left(\alpha_{\infty}\right)} e^{-i t \mathcal{H}\left(\alpha_{\infty}\right)} U_{1}
$$

exists as a strong $L^{2}$ limit. Indeed, this follows from

$$
\int_{0}^{\infty}\left\|e^{i s \mathcal{H}_{0}\left(\alpha_{\infty}\right)} V e^{-i s \mathcal{H}\left(\alpha_{\infty}\right)} U_{1}\right\|_{2} d s \lesssim \int_{0}^{\infty}\left\|\langle x\rangle^{-\theta} e^{-i s \mathcal{H}\left(\alpha_{\infty}\right)} U_{1}\right\|_{\infty} d s
$$

with the latter integral being controlled as follows:

$$
\begin{aligned}
& \left\|\langle x\rangle^{-\theta} e^{-i s \mathcal{H}\left(\alpha_{\infty}\right)} U_{1}\right\|_{\infty} \\
& =\left\|\langle x\rangle^{-\theta}\left[e^{-i s \mathcal{H}\left(\alpha_{\infty}\right)} U_{d i s}(0)-i \int_{0}^{\infty} e^{i(r-s) \mathcal{H}\left(\alpha_{\infty}\right)} P_{s}\left(\alpha_{\infty}\right) F(r) d r\right]\right\|_{\infty} .
\end{aligned}
$$


The first term on the right is controlled by Corollary 8.3 as well as Sobolev's inequality:

$$
\left\|\langle x\rangle^{-\theta} e^{-i s \mathcal{H}\left(\alpha_{\infty}\right)} U_{\text {dis }}(0)\right\|_{L_{x}^{\infty}} \lesssim\langle s\rangle^{-1-\epsilon} .
$$

For the integral term we rewrite it as

$$
\begin{aligned}
& \left\|\langle x\rangle^{-\theta} \int_{0}^{\infty} e^{i(r-s) \mathcal{H}\left(\alpha_{\infty}\right)} P_{s}\left(\alpha_{\infty}\right)[F(r)] d r\right\|_{\infty} \\
& =\left\|\langle x\rangle^{-\theta} \int_{0}^{\infty} e^{i(r-s) \mathcal{H}\left(\alpha_{\infty}\right)} P_{s}\left(\alpha_{\infty}\right)\left[i \pi \partial_{\pi} \tilde{W}_{\infty}(\pi)(r)+N_{\infty}(U, \pi)(r)+V_{\infty} U(r)\right] d r\right\|_{\infty} .
\end{aligned}
$$

Proceeding as in the proof of Proposition 4.4, etc., and keeping the a priori estimates in mind, we easily bound this integral by

$$
\lesssim \int_{0}^{\infty} \min \left\{|r-s|^{-1-\epsilon},|r-s|^{-\frac{1}{2}}\right\}\langle r\rangle^{-1-\epsilon} d r
$$

Putting the preceding observations together, we obtain that

$$
\begin{aligned}
& \int_{0}^{\infty}\left\|\langle x\rangle^{-\theta} e^{-i s \mathcal{H}\left(\alpha_{\infty}\right)} U_{1}\right\|_{\infty} d s \\
& \lesssim \int_{0}^{\infty}\left[\langle s\rangle^{-1-\epsilon}+\int_{0}^{\infty} \min \left\{|r-s|^{-1-\epsilon},|r-s|^{-\frac{1}{2}}\right\}\langle r\rangle^{-1-\epsilon} d r\right] d s<\infty
\end{aligned}
$$

as desired. It follows that

$$
U(t)=e^{-i t \mathcal{H}_{0}\left(\alpha_{\infty}\right)} U_{2}+o_{L^{2}}(1) .
$$

Finally,

$$
Z(t)=\mathcal{G}_{\infty}(t)^{-1} M(t)^{-1} U(t)=e^{-i t \mathcal{H}_{0}} \mathcal{G}_{\infty}^{-1}(0) U_{2}+o_{L^{2}}(1),
$$

where $\mathcal{H}_{0}=\left(\begin{array}{cc}-\partial_{x}^{2} & 0 \\ 0 & \partial_{x}^{2}\end{array}\right)$. Setting $\mathcal{G}_{\infty}^{-1}(0) U_{2}=\left(\begin{array}{l}f_{0} \\ \bar{f}_{0}\end{array}\right)$ and $Z(t)=\left(\begin{array}{l}R(t) \\ \bar{R}(t)\end{array}\right)$, we obtain

$$
R(t)=e^{i t \partial_{x}^{2}} f_{0}+o_{L^{2}}(1)
$$

and the theorem is proved.

Proof of Theorem 1.2, The idea is as follows: Given $\alpha_{0}$, consider the NLS equation (1.1) with initial data $\phi\left(\cdot, \alpha_{0}\right)+R_{0}$. Applying the usual four-parameter family of symmetries (Galilei giving three parameters, scaling one - scaling here is the same as the parameter $\alpha$ ), we transform this to $W(0, \cdot)+R_{1}$ where $W(0, x)$ is a soliton with a general parameter vector $\pi_{0}$ which is close to $\left(0,0,0, \alpha_{0}\right)$. Hence, we can apply Theorem 1.1 to conclude that these initial data will give rise to global solutions with the desired properties as long as $W(0, x)+R_{1}$ lies on the stable manifold associated with $W(0, x)$. To prove that we obtain four dimensions back in this fashion requires checking that the derivatives of $W(0, x)$ in its parameters are transverse to the linear space $\mathcal{S}$ of Theorem 1.1. However, these derivatives are basically the elements of the root space $\mathcal{N}$ of $\mathcal{H}\left(\alpha_{0}\right)$, whereas we know that $\mathcal{S}$ is perpendicular to the root space $\mathcal{N}^{*}$ of $\mathcal{H}\left(\alpha_{0}\right)^{*}$. More precisely, it is easily verified that these derivatives are

$$
\left(\begin{array}{c}
\partial_{\alpha} \phi \\
\partial_{\alpha} \phi
\end{array}\right),\left(\begin{array}{c}
i \phi \\
i \phi
\end{array}\right),\left(\begin{array}{c}
i x \phi \\
-i x \phi
\end{array}\right),\left(\begin{array}{c}
\partial_{x} \phi \\
\partial_{x} \phi
\end{array}\right) .
$$

But Lemma 3.3 implies that no nonzero vector in $\mathcal{N}$ is perpendicular to $\mathcal{N}^{*}$, which proves that $\mathcal{N}$ is transverse to $\mathcal{S}$, as desired. 


\section{The SCATTERING THEORY FOR SCHRÖDINGER SYSTEMS}

This section presents the scattering theory for matrix Hamiltonians on the line which was developed by Buslaev and Perelman [5]. Since the presentation in [5] is somewhat sketchy and since we need to refine some of the estimates in [5], we give full details.

Definition 5.1. In what follows,

$$
\mathcal{H}_{0}:=\left(\begin{array}{cc}
-\partial_{x x}+1 & 0 \\
0 & \partial_{x x}-1
\end{array}\right), V(x)=\left(\begin{array}{cc}
V_{1}(x) & V_{2}(x) \\
-V_{2}(x) & -V_{1}(x)
\end{array}\right), \mathcal{H}:=\mathcal{H}_{0}+V .
$$

We will assume that $V$ as well as all its derivatives are exponentially decaying:

$$
\left\|V^{(k)}(x)\right\| \leq C_{k} e^{-\gamma|x|} \quad \forall k \geq 0
$$

with some $0<\gamma<1$. Moreover, all entries of $V$ are real-valued, and we will also assume that $V$ is even: $V(x)=V(-x)$.

The decay and regularity assumptions can be relaxed to polynomial decay and a finite number of derivatives, but we do not dwell on this issue. Let the usual Pauli matrices be given by

$$
\sigma_{1}=\left(\begin{array}{cc}
0 & 1 \\
1 & 0
\end{array}\right), \sigma_{2}=\left(\begin{array}{cc}
0 & -i \\
i & 0
\end{array}\right), \sigma_{3}=\left(\begin{array}{cc}
1 & 0 \\
0 & -1
\end{array}\right) .
$$

Note that any $V$ as in Definition 5.1 satisfies

$$
\sigma_{3} \mathcal{H}^{*} \sigma_{3}=\mathcal{H}, \sigma_{1} \mathcal{H} \sigma_{1}=-\mathcal{H}
$$

The following three lemmas construct a basis of the solution space to $\mathcal{H} f=\left(\lambda^{2}+1\right) f$ with prescribed asymptotics at infinity. These are of course analogues of the Jost solutions in the scalar case. Throughout, $\mu=\sqrt{\lambda^{2}+2}$.

Lemma 5.2. For every $\lambda \in \mathbb{R}$ there exists a solution $f_{3}(x, \lambda)$ of the equation

$$
\mathcal{H} f_{3}(\cdot, \lambda)=\left(\lambda^{2}+1\right) f_{3}(\cdot, \lambda)
$$

with the property that $f_{3}(x, \lambda) \sim e^{-\mu x}\left(\begin{array}{l}0 \\ 1\end{array}\right)$ as $x \rightarrow \infty$. Moreover, $f_{3}$ is smooth in both variables and satisfies the estimates

$$
\left|\partial_{\lambda}^{\ell} \partial_{x}^{k}\left[e^{\mu x} f_{3}(x, \lambda)-\left(\begin{array}{l}
0 \\
1
\end{array}\right)\right]\right| \leq C_{k} \mu^{-1-\ell} e^{-\gamma x}
$$

for all $x \geq 0$ and $k, \ell \geq 0$. Finally, $\sup _{\lambda \in \mathbb{R}} \sup _{x \in \mathbb{R}}\left|e^{\mu x} f_{3}(x, \lambda)\right| \leq C(V)$.

Proof. We set

$$
f_{3}(x, \lambda):=e^{-\mu x}\left(\begin{array}{l}
0 \\
1
\end{array}\right)+\int_{x}^{\infty}\left(\begin{array}{cc}
\frac{\sin (\lambda(y-x))}{\lambda} & 0 \\
0 & -\frac{\sinh (\mu(y-x))}{\mu}
\end{array}\right) V(y) f_{3}(y, \lambda) d y
$$

Equivalently, with

$$
K(x, y ; \lambda):=\left(\begin{array}{cc}
\frac{\sin (\lambda(y-x))}{\lambda} & 0 \\
0 & -\frac{\sinh (\mu(y-x))}{\mu}
\end{array}\right) e^{\mu(x-y)}
$$

we have

$$
e^{\mu x} f_{3}(x, \lambda)=\left(\begin{array}{l}
0 \\
1
\end{array}\right)+\int_{x}^{\infty} K(x, y ; \lambda) V(y) e^{\mu y} f_{3}(y, \lambda) d y .
$$


Since for all $\lambda \in \mathbb{R}$

$$
\sup _{y \geq x}|K(x, y ; \lambda)| \leq \sup _{y \geq x}\left\{(y-x) e^{\mu(x-y)}+\frac{\sinh (\mu(x-y))}{\mu} e^{\mu(x-y)}\right\} \leq C \mu^{-1},
$$

with a universal constant $C$, we conclude that $e^{\mu x} f_{3}(\cdot, \lambda)$ solves a Volterra integral equation and thus

$$
\sup _{\lambda \in \mathbb{R}} \sup _{x \in \mathbb{R}}\left|e^{\mu x} f_{3}(x, \lambda)\right| \leq C(V) .
$$

Thus, we obtain that

$$
\left|e^{\mu x} f_{3}(x, \lambda)-\left(\begin{array}{l}
0 \\
1
\end{array}\right)\right| \leq \int_{x}^{\infty} C \mu^{-1} e^{-\gamma y} d y \leq C \mu^{-1} e^{-\gamma x}
$$

for all $x \geq 0$. The estimate (5.3) follows by differentiating the Volterra equation (5.6). Indeed, since $K(x, x, \lambda)=0$ and $\partial_{x} K(x, y ; \lambda)=-\partial_{y} K(x, y ; \lambda)$, integration by parts yields

$$
\begin{aligned}
\partial_{x}\left(e^{\mu x} f_{3}(x, \lambda)\right) & =\int_{x}^{\infty} K(x, y ; \lambda) V^{\prime}(y) e^{\mu y} f_{3}(y, \lambda) d y \\
& +\int_{x}^{\infty} K(x, y ; \lambda) V(y) \partial_{y}\left(e^{\mu y} f_{3}(y, \lambda)\right) d y .
\end{aligned}
$$

By the usual estimates for Volterra equations as well as (5.1) and (5.7),

$$
\left|\partial_{x}\left(e^{\mu x} f_{3}(x, \lambda)\right)\right| \leq C \mu^{-1} \int_{x}^{\infty} e^{-\gamma y} d y \leq C \mu^{-1} e^{-\gamma x},
$$

for $x \geq 0$, as claimed. The higher derivatives in $x$ follow in a similar fashion. Indeed, integrating by parts, one verifies inductively that

$$
\partial_{x}^{k}\left(e^{\mu x} f_{3}(x, \lambda)\right)=\sum_{j=0}^{k}\left(\begin{array}{c}
k \\
j
\end{array}\right) \int_{x}^{\infty} V^{(k-j)}(y) \partial_{y}^{j}\left[e^{\mu y} f_{3}(y, \lambda)\right] d y,
$$

which implies the bounds

$$
\left|\partial_{x}^{k}\left(e^{\mu x} f_{3}(x, \lambda)\right)\right| \leq C_{k} \mu^{-1} e^{-\gamma x}
$$

for all $x \geq 0$. As far as the derivatives in $\lambda$ are concerned, differentiating (5.5) in $\lambda$ reveals that

$$
\sup _{y \geq x}\left|\partial_{\lambda}^{\ell} K(x, y ; \lambda)\right| \leq C_{\ell} \mu^{-\ell-1}
$$

for all $\ell \geq 0$. Apply $\partial_{\lambda}^{\ell}$ to (5.8). Induction in $\ell$ implies the estimate (5.3).

Next, we find a pair of oscillatory solutions.

Lemma 5.3. For all $\lambda \in \mathbb{R}$ there exist solutions $f_{1}(\cdot, \lambda), f_{2}(\cdot, \lambda)$ of

$$
\mathcal{H} f_{j}(\cdot, \lambda)=\left(1+\lambda^{2}\right) f_{j}(\cdot, \lambda)
$$

$(j=1,2)$ and with the property that $f_{2}(\cdot, \lambda)=\overline{f_{1}(\cdot, \lambda)}$ and

$$
f_{1}(x, \lambda)=\left(\begin{array}{c}
e^{i x \lambda} \\
0
\end{array}\right)+O\left(\mu^{-1} e^{-\gamma x}\right)
$$


as $x \rightarrow \infty$. The constant in the $O$-term is uniform in $\lambda \in \mathbb{R}$. Moreover, $f_{1}$ is smooth in both variables and there exists a constan $19 x_{0} \geq 0$ only depending on $V$ such that

$$
\left|\partial_{\lambda}^{\ell} \partial_{x}^{k}\left[e^{-i \lambda x} f_{1}(x, \lambda)-\left(\begin{array}{l}
1 \\
0
\end{array}\right)\right]\right| \leq C_{k, \ell} \mu^{-1+k} x^{\ell} e^{-\gamma x}
$$

for all $k, \ell \geq 0, \lambda \in \mathbb{R}$, and $x \geq x_{0}$. Finally, the same bound holds for all $x \geq 0$ provided $|\lambda| \geq \lambda_{0}$ where $\lambda_{0} \geq 0$ is some constant ${ }^{19}$ that only depends on $V$.

Proof. Since $V$ has real entries, any solution $f_{1}(\cdot, \lambda)$ gives rise to another solution $\overline{f_{2}(\cdot, \lambda)}$. Hence it will suffice to find $f_{1}$. We seek a solution of the form

$$
f_{1}(x, \lambda)=\left(\begin{array}{l}
1 \\
0
\end{array}\right) v(x, \lambda)+f_{3}(x, \lambda) u(x, \lambda)
$$

where $v(x, \lambda) \sim e^{i x \lambda}$, and $f_{3}(x, \lambda)=\left(\begin{array}{l}f_{3}^{(1)}(x, \lambda) \\ f_{3}^{(2)}(x, \lambda)\end{array}\right)$ is as in Lemma 5.2. Clearly,

$$
\begin{aligned}
\mathcal{H}\left(f_{3}(\cdot, \lambda) u\right) & =u \mathcal{H}\left(f_{3}(\cdot, \lambda)\right)+\left(\begin{array}{c}
-\partial_{x x}\left(u f_{3}^{(1)}(\cdot, \lambda)\right)+u \partial_{x x} f_{3}^{(1)}(\cdot, \lambda) \\
\partial_{x x}\left(u f_{3}^{(1)}(\cdot, \lambda)\right)-u \partial_{x x} f_{3}^{(1)}(\cdot, \lambda)
\end{array}\right) \\
& =u\left(1+\lambda^{2}\right) f_{3}(\cdot, \lambda)+\left(\begin{array}{c}
-\partial_{x x}\left(u f_{3}^{(1)}(\cdot, \lambda)\right)+u \partial_{x x} f_{3}^{(1)}(\cdot, \lambda) \\
\partial_{x x}\left(u f_{3}^{(1)}(\cdot, \lambda)\right)-u \partial_{x x} f_{3}^{(1)}(\cdot, \lambda)
\end{array}\right) .
\end{aligned}
$$

In order to have $\mathcal{H} f_{1}(\cdot, \lambda)=\left(1+\lambda^{2}\right) f_{1}(\cdot, \lambda)$, we therefore need

$0=\left(\mathcal{H}-\left(\lambda^{2}+1\right)\right) f_{1}(\cdot, \lambda)$

$$
=\left(\begin{array}{c}
\left(-\partial_{x x}-\lambda^{2}+V_{11}\right) v(\cdot, \lambda) \\
V_{21} v(\cdot, \lambda)
\end{array}\right)+\left(\begin{array}{c}
-\partial_{x x} u(\cdot, \lambda) f_{3}^{(1)}(\cdot, \lambda)-2 \partial_{x} u(\cdot, \lambda) \partial_{x} f_{3}^{(1)}(\cdot, \lambda) \\
\partial_{x x} u(\cdot, \lambda) f_{3}^{(2)}(\cdot, \lambda)+2 \partial_{x} u(\cdot, \lambda) \partial_{x} f_{3}^{(2)}(\cdot, \lambda)
\end{array}\right) .
$$

The homogeneous equation

$$
y^{\prime \prime} f_{3}^{(2)}(\cdot, \lambda)+2 y^{\prime} \partial_{x} f_{3}^{(2)}(\cdot, \lambda)=0
$$

has the solution

$$
y^{\prime}(x)=C\left(f_{3}^{(2)}(x, \lambda)\right)^{-2}
$$

which is well defined provided $x \geq x_{0}$ by Lemma 5.2. Here $x_{0} \geq 0$ is a large constant independent of $\lambda$ (for large $|\lambda|$, we can take $x_{0}=0$ ). From the second coordinate in (5.11) and the usual "variation of constants" method we obtain that

$$
C^{\prime}(x)=-f_{3}^{(2)}(x, \lambda) V_{21}(x) v(x, \lambda)
$$

which implies, together with the boundary condition $C(\infty)=0$, that

$$
u^{\prime}(x, \lambda)=\left[f_{3}^{(2)}(x, \lambda)\right]^{-2} \int_{x}^{\infty} f_{3}^{(2)}(y, \lambda) V_{21}(y) v(y, \lambda) d y .
$$

\footnotetext{
${ }^{19}$ This constant becomes large as $V$ becomes large.
} 
From the first coordinate in (5.11) we conclude that (dropping $\lambda$ from $f_{3}, v, u$ for simplicity)

$$
\begin{aligned}
v(x)= & e^{i x \lambda}-\int_{x}^{\infty} \frac{\sin (\lambda(y-x))}{\lambda}\left(u^{\prime \prime}(y) f_{3}^{(1)}(y)+2 u^{\prime}(y) f_{3}^{(1)}(y)^{\prime}-V_{11}(y) v(y)\right) d y \\
= & e^{i x \lambda}+\int_{x}^{\infty} \frac{\sin (\lambda(y-x))}{\lambda}\left(V_{21}(y) \frac{f_{3}^{(1)}}{f_{3}^{(2)}}(y)+V_{11}(y)\right) v(y) d y \\
- & 2 \int_{x}^{\infty} \frac{\sin (\lambda(y-x))}{\lambda}\left(-\frac{f_{3}^{(2)}(y)^{\prime}}{f_{3}^{(2)}(y)} f_{3}^{(1)}(y)+f_{3}^{(1)}(y)^{\prime}\right)\left[f_{3}^{(2)}(y)\right]^{-2} \\
& \times \int_{y}^{\infty} f_{3}^{(2)}(z) V_{21}(z) v(z) d z d y
\end{aligned}
$$

This can be written as

$$
v(x, \lambda)=e^{i x \lambda}+\int_{x}^{\infty} K(x, y ; \lambda) v(y, \lambda) d y
$$

where $K(x, y ; \lambda):=K_{1}(x, y ; \lambda)+K_{2}(x, y ; \lambda)$ according to the splitting

$$
\begin{aligned}
K_{1}(x, y ; \lambda)= & \frac{\sin (\lambda(y-x))}{\lambda}\left(V_{21}(y) \frac{f_{3}^{(1)}}{f_{3}^{(2)}}(y ; \lambda)+V_{11}(y)\right), \\
K_{2}(x, y ; \lambda)= & -2 \int_{x}^{y} \frac{\sin (\lambda(z-x))}{\lambda}\left(-\frac{f_{3}^{(2)}(z ; \lambda)^{\prime}}{f_{3}^{(2)}(z ; \lambda)} f_{3}^{(1)}(z ; \lambda)+f_{3}^{(1)}(z ; \lambda)^{\prime}\right) \\
& \times\left[f_{3}^{(2)}(z ; \lambda)\right]^{-2} d z f_{3}^{(2)}(y ; \lambda) V_{21}(y) .
\end{aligned}
$$

Since $y \geq x \geq x_{0} \geq 0$, Lemma 5.2 implies that

$$
\begin{aligned}
\left|K_{1}(x, y ; \lambda)\right| & \leq C(y-x)(1+|\lambda|(y-x))^{-1} e^{-\gamma y} \\
\left|K_{2}(x, y ; \lambda)\right| & \leq \int_{x}^{y}(z-x)(1+|\lambda|(z-x))^{-1} e^{(\mu-\gamma) z} d z e^{-(\mu+\gamma) z} \\
& \leq C \frac{y-x}{1+|\lambda|(y-x)} \mu^{-1} e^{-2 \gamma y} .
\end{aligned}
$$

In the final estimate we used that $\frac{a}{1+|\lambda| a}$ is increasing in $a$. Hence, (5.13) is a Volterra equation with a solution $v(x, \lambda)$ on the interval $\left[x_{0}, \infty\right)$ satisfying

$$
\begin{aligned}
\left|v(x, \lambda)-e^{i x \lambda}\right| & \leq C \int_{x}^{\infty} \frac{y-x}{1+|\lambda|(y-x)} e^{-\gamma y} d y=C e^{-\gamma x} \int_{0}^{\infty} \frac{u}{1+|\lambda| u} e^{-\gamma u} d u \\
& \leq C(1+|\lambda|)^{-1} e^{-\gamma x}
\end{aligned}
$$

for all $x \geq x_{0}$. Thus, in view of (5.12),

$$
\left|u^{\prime}(x, \lambda)\right| \leq C e^{2 \mu x} \int_{x}^{\infty} e^{-(\mu+\gamma) y} d y \leq C \mu^{-1} e^{(\mu-\gamma) x}
$$

for all $x \geq x_{0}$ and $\lambda \in \mathbb{R}$. Hence, assuming that $u\left(x_{0}, \lambda\right)=0$, we obtain that

$$
|u(x, \lambda)| \leq C \mu^{-2} e^{(\mu-\gamma) x} .
$$

By the preceding,

$$
\left|f_{1}(x, \lambda)-\left(\begin{array}{c}
e^{i x \lambda} \\
0
\end{array}\right)\right| \leq C \mu^{-1} e^{-\gamma x}
$$


with a constant $C$ which is uniform in $x \geq x_{0}$ and $\lambda \in \mathbb{R}$. Now continue $f_{1}(\cdot, \lambda)$ to the left of $x_{0}$ by means of the existence and uniqueness theorem.

As far as the derivatives are concerned, set

$$
\tilde{K}(x, y ; \lambda):=e^{-i \lambda(x-y)} K(x, y ; \lambda), \quad \tilde{v}(x, \lambda):=e^{-i \lambda x} v(x, \lambda),
$$

and similarly with $\tilde{K}_{1}, \tilde{K}_{2}$; see (5.14), (5.15). Then

$$
\partial_{\lambda} \tilde{v}(x, \lambda)=\int_{x}^{\infty} \partial_{\lambda} \tilde{K}(x, y ; \lambda) \tilde{v}(y, \lambda) d y+\int_{x}^{\infty} \tilde{K}(x, y ; \lambda) \partial_{\lambda} \tilde{v}(y, \lambda) d y .
$$

In view of (5.3),

$$
\left|\partial_{\lambda} f_{3}(x, \lambda)\right| \leq C x e^{-\mu x}
$$

for large $x$. Consequently, for $y \geq x \geq x_{0}$,

$$
\begin{aligned}
\left|\partial_{\lambda} \tilde{K}_{1}(x, y ; \lambda)\right| & =\left|\partial_{\lambda}\left[e^{-i \lambda(x-y)} \frac{\sin (\lambda(y-x))}{\lambda}\left(V_{21}(y) \frac{f_{3}^{(1)}}{f_{3}^{(2)}}(y ; \lambda)+V_{11}(y)\right)\right]\right| \\
& \leq C \frac{(y-x)^{2}}{1+|\lambda|(y-x)} e^{-\gamma y}+C \mu^{-1} \frac{y-x}{1+|\lambda|(y-x)} y e^{-2 \gamma y} .
\end{aligned}
$$

To obtain this bound, it is helpful to introduce $\phi(u):=\frac{\sin (u)}{u}$. Then $\left|\phi^{(k)}(u)\right| \leq$ $C_{k}(1+|u|)^{-1}$ for all $k \geq 0$ and

$$
\frac{\sin (\lambda(y-x))}{\lambda}=(y-x) \phi(\lambda(y-x)) \text {. }
$$

Similarly,

$$
\begin{aligned}
& \left|\partial_{\lambda} \tilde{K}_{2}(x, y ; \lambda)\right| \\
& =\mid \partial_{\lambda} \int_{x}^{y} e^{-i \lambda(x-y)} \frac{\sin (\lambda(z-x))}{\lambda}\left(-\frac{f_{3}^{(2)}(z ; \lambda)^{\prime}}{f_{3}^{(2)}(z ; \lambda)} f_{3}^{(1)}(z ; \lambda)+f_{3}^{(1)}(z ; \lambda)^{\prime}\right) \\
& \quad \times\left[f_{3}^{(2)}(z ; \lambda)\right]^{-2} d z f_{3}^{(2)}(y ; \lambda) V_{21}(y) \mid \\
& \leq C \int_{x}^{y} \frac{(y-x)^{2}}{1+|\lambda|(y-x)} e^{-(\mu+\gamma) z} e^{2 \mu z} d z e^{-(\mu+\gamma) y} \\
& +C \int_{x}^{y} \frac{y-x}{1+|\lambda|(y-x)}(1+\mu z) \mu^{-1} e^{-(\mu+\gamma) z} e^{2 \mu z} d z e^{-(\mu+\gamma) y} \\
& \leq C \frac{(y-x)^{2}}{1+|\lambda|(y-x)} \mu^{-1} e^{-2 \gamma y}+C \frac{y-x}{1+|\lambda|(y-x)} \mu^{-1} y e^{-2 \gamma y} .
\end{aligned}
$$

The conclusion is that

$$
\left|\int_{x}^{\infty} \partial_{\lambda} \tilde{K}(x, y ; \lambda) \tilde{v}(y, \lambda) d y\right| \leq C \mu^{-1} e^{-\gamma x}
$$

and therefore also

$$
\left|\partial_{\lambda} \tilde{v}(x, \lambda)\right| \leq C \mu^{-1} e^{-\gamma x}
$$

see (5.16). Inserting this into (5.10) yields

$$
\left|\partial_{\lambda}\left[e^{-i x \lambda} f_{1}(x, \lambda)\right]\right| \leq C \mu^{-1} x e^{-\gamma x}
$$

for all $x \geq x_{0}$. The case of higher derivatives in $\lambda$ is similar. Indeed,

$$
\partial_{\lambda}^{\ell} \tilde{v}(x, \lambda)=\sum_{j=0}^{\ell}\left(\begin{array}{l}
\ell \\
j
\end{array}\right) \int_{x}^{\infty} \partial_{\lambda}^{j} \tilde{K}(x, y ; \lambda) \partial_{\lambda}^{\ell-j} \tilde{v}(y, \lambda) d y .
$$


As before, Lemma 5.2 leads to the bounds

$$
\left|\partial_{\lambda}^{\ell} \tilde{K}(x, y ; \lambda)\right| \leq C_{\ell} \frac{(y-x)^{\ell+1}+y-x}{1+|\lambda|(y-x)} e^{-\gamma y} .
$$

In combination with (5.17), this estimate inductively yields

$$
\left|\partial_{\lambda}^{\ell} \tilde{v}(x, \lambda)\right| \leq C_{\ell} \mu^{-1} e^{-\gamma x} \text { or }\left|\partial_{\lambda}^{\ell} v(x, \lambda)\right| \leq C_{\ell} x^{\ell} \mu^{-1} e^{-\gamma x}
$$

for large $x$. Inserting this bound into the defining equation (5.12) for $u^{\prime}$ implies that

$$
\left|\partial_{\lambda}^{\ell} u^{\prime}(x, \lambda)\right| \leq C_{\ell} x^{\ell} \mu^{-1} e^{(\mu-\gamma) x} .
$$

Since $\partial_{\lambda}^{\ell} u\left(x_{0}, \lambda\right)=0$ for all $\lambda$,

$$
\left|\partial_{\lambda}^{\ell} u(x, \lambda)\right| \leq C_{\ell} x^{\ell} \mu^{-2} e^{(\mu-\gamma) x} .
$$

Finally, inserting this estimate into (5.10), we obtain

$$
\left|\partial_{\lambda}^{\ell}\left[e^{-i x \lambda} f_{1}(x, \lambda)\right]\right| \leq C_{\ell} x^{\ell} \mu^{-1} e^{-\gamma x},
$$

which is (5.9) with $k=0$. The case of $x$-derivatives, i.e., $k \geq 1$ in (5.9), follows by similar considerations. We skip the details.

Remark 5.4. As already noted, we can take $x_{0}=0$ in the previous proof for large $|\lambda|$. This allows us to state that

$$
\sup _{x \geq 0}\left|\partial_{x} f_{1}(x, \lambda)-i \lambda\left(\begin{array}{c}
e^{i x \lambda} \\
0
\end{array}\right)\right| \leq C
$$

for large $|\lambda|$, which will be useful later. Another important (but simple) observation concerns the point $\lambda=0$. There $f_{1}(\cdot, \lambda), f_{2}(\cdot, \lambda)$ are identical. However, it is simple to obtain a pair of linearly independent solutions at $\lambda=0$. Indeed, just take $f_{1}(\cdot, 0)$ and $\partial_{\lambda} f_{1}(\cdot, 0)$. Note that the asymptotic behavior of $\partial_{\lambda} f_{1}(\cdot, 0)$ is $i x$ as $x \rightarrow \infty$. Alternatively, one can also work with the pair

$$
f_{1}(\cdot, \lambda), \quad \frac{f_{1}(\cdot, \lambda)-f_{2}(\cdot,-\lambda)}{\lambda}
$$

which is independent for all $\lambda \in \mathbb{R}$.

Next, we construct an exponentially growing solution at $+\infty$. We will later modify $\tilde{f}_{4}$ to obtain $f_{4}$, hence the notation.

Lemma 5.5. There exists a solution $\tilde{f}_{4}(\cdot, \lambda)$ of $\mathcal{H} \tilde{f}_{4}(\cdot, \lambda)=\left(1+\lambda^{2}\right) \tilde{f}_{4}(\cdot, \lambda)$ with the property that

$$
\tilde{f}_{4}(\cdot, \lambda)=e^{\mu x}\left(\begin{array}{l}
0 \\
1
\end{array}\right)+O\left((1+|\lambda|)^{-1} e^{(\mu-\gamma) x}\right)
$$

as $x \rightarrow \infty$. The constant in the $O$-term is uniform in $\lambda$. Moreover, $f_{4}(\cdot, \lambda)$ is a smooth function of its arguments and there exists a constan $x_{1} \geq 0$ only depending on $V$ such that

$$
\left|\partial_{\lambda}^{\ell} \partial_{x}^{k}\left[e^{-\mu x} \tilde{f}_{4}(x, \lambda)-\left(\begin{array}{l}
0 \\
1
\end{array}\right)\right]\right| \leq C_{k, \ell} \mu^{-1+k} x^{\ell} e^{-\gamma x}
$$

for all $k, \ell \geq 0, \lambda \in \mathbb{R}$, and $x \geq x_{1}$. Finally, the same bound holds for all $x \geq 0$ provided $|\lambda| \geq \lambda_{1}$ where $\lambda_{1} \geq 0$ is some constant ${ }^{20}$ that only depends on $V$.

\footnotetext{
${ }^{20}$ This constant becomes large as $V$ becomes large.
} 
Proof. Make the ansatz

$$
\begin{aligned}
\tilde{f}_{4}(x, \lambda) & =e^{\mu x}\left(\begin{array}{l}
0 \\
1
\end{array}\right)+\int_{x}^{\infty}\left(\begin{array}{cc}
0 & 0 \\
0 & -\frac{1}{2 \mu} e^{\mu(x-y)}
\end{array}\right) V(y) \tilde{f}_{4}(y, \lambda) d y \\
& +\int_{x_{1}}^{x}\left(\begin{array}{cc}
\frac{\sin (\lambda(x-y))}{\lambda} & 0 \\
0 & -\frac{1}{2 \mu} e^{-\mu(x-y)}
\end{array}\right) V(y) \tilde{f}_{4}(y, \lambda) d y
\end{aligned}
$$

where $x_{1} \geq 0$ is some constant that will be chosen large. Clearly, if $\tilde{f}_{4}(x, \lambda)$ grows at most like $e^{\mu x}$ as $x \rightarrow \infty$, then this integral equation is well defined. Moreover, it is easy to check that a solution of this integral equation satisfies $\mathcal{H} \tilde{f}_{4}(\cdot, \lambda)=$ $\left(1+\lambda^{2}\right) \tilde{f}_{4}(\cdot, \lambda)$. To find a solution, we solve

$$
\begin{aligned}
f(x, \lambda) & =\left(\begin{array}{l}
0 \\
1
\end{array}\right)+\int_{x}^{\infty}\left(\begin{array}{cc}
0 & 0 \\
0 & -\frac{1}{2 \mu}
\end{array}\right) V(y) f(y, \lambda) d y \\
& +\int_{x_{1}}^{x}\left(\begin{array}{cc}
\frac{\sin (\lambda(x-y))}{\lambda} e^{-\mu(x-y)} & 0 \\
0 & -\frac{1}{2 \mu} e^{-2 \mu(x-y)}
\end{array}\right) V(y) f(y, \lambda) d y
\end{aligned}
$$

by the contraction principle. Thus, $\tilde{f}_{4}(x, \lambda):=e^{\mu x} f(x, \lambda)$ will be the desired solution. Denote the right-hand side by $T$. Then

$$
\begin{aligned}
(T f-T g)(x) & =\int_{x}^{\infty}\left(\begin{array}{cc}
0 & 0 \\
0 & -\frac{1}{2 \mu}
\end{array}\right) V(y)[f(y)-g(y)] d y \\
& +\int_{x_{1}}^{x}\left(\begin{array}{cc}
\frac{\sin (\lambda(x-y))}{\lambda} e^{-\mu(x-y)} & 0
\end{array}\right) V(y)[f(y)-g(y)] d y,
\end{aligned}
$$

which implies that

$$
\begin{aligned}
& |(T f-T g)(x)| \leq C(1+|\lambda|)^{-1} \int_{x}^{\infty} e^{-\gamma y}|f(y)-g(y)| d y \\
& +C(1+|\lambda|)^{-1} \int_{x_{1}}^{x}\left[\mu(x-y) e^{-\mu(x-y)}+e^{-2 \mu(x-y)}\right] e^{-\gamma y}|f(y)-g(y)| d y \\
& \leq C(1+|\lambda|)^{-1} e^{-\gamma x_{1}} \sup _{y \geq x_{1}}|f(y)-g(y)|
\end{aligned}
$$

for all $x \geq x_{1}$. Hence, $T$ is a contraction in

$$
\left\{f \in C\left(\left[x_{1}, \infty\right), \mathbb{C}^{2}\right)\left|\sup _{x \geq x_{1}}\right| f(x) \mid \leq 2\right\}
$$

provided $x_{1}$ is large (if $|\lambda|$ is large, we can take $x_{1}=0$ ). If $f(\cdot, \lambda)$ is the fixed-point of $T$, then

$$
\left|f(x, \lambda)-\left(\begin{array}{l}
0 \\
1
\end{array}\right)\right| \leq C(1+|\lambda|)^{-1} e^{-\gamma x},
$$

for all $x \geq x_{1}$. The estimate on the derivatives follows by differentiating the equation (5.20).

Remark 5.6. As noted, for large $|\lambda|$, we can take $x_{1}=0$ in the previous proof. This allows us to state that

$$
\left|\tilde{f}_{4}(0, \lambda)-\left(\begin{array}{l}
0 \\
1
\end{array}\right)\right| \leq C|\lambda|^{-1},
$$


as well as

for large $|\lambda|$.

$$
\left|\partial_{x} \tilde{f}_{4}(0, \lambda)-\left(\begin{array}{l}
0 \\
\mu
\end{array}\right)\right| \leq C
$$

Here we record a useful property of these solutions.

Corollary 5.7. The solutions $f_{1}, f_{2}, f_{3}, \tilde{f}_{4}$ from the previous lemmas satisfy

$$
\begin{array}{ll}
f_{1}(\cdot,-\lambda)=\overline{f_{1}(\cdot, \lambda)}=f_{2}(\cdot, \lambda), \quad f_{2}(\cdot,-\lambda)=\overline{f_{2}(\cdot, \lambda)}=f_{1}(\cdot, \lambda), \\
f_{3}(\cdot,-\lambda)=\overline{f_{3}(\cdot, \lambda)}=f_{3}(\cdot, \lambda), \quad & \tilde{f}_{4}(\cdot,-\lambda)=\overline{\tilde{f}_{4}(\cdot, \lambda)}=\tilde{f}_{4}(\cdot, \lambda)
\end{array}
$$

for all $\lambda \in \mathbb{R}$. Moreover, $f_{1}(\cdot, 0)=f_{2}(\cdot, 0)$.

Proof. This can be seen by inspecting the various integral equations defining these solutions. Indeed, since $V$ has real entries, (5.4) and (5.19) are invariant under both conjugation and the substitution $\lambda \rightarrow-\lambda$. Finally, (5.10), (5.12), and (5.13) imply that conjugation of $f_{1}(\cdot, \lambda)$ is equivalent to $\lambda \rightarrow-\lambda$.

The following two lemmas introduce the Wronskian in the matrix context. We will need to use the property that $\sigma_{3} V^{*} \sigma_{3}=V$; see Definition 5.1 and (5.2).

Lemma 5.8. For two differentiable functions $f, g$ taking values in $\mathbb{C}^{2}$ let

$$
W[f, g](x):=\left\langle f^{\prime}(x), g(x)\right\rangle-\left\langle f(x), g^{\prime}(x)\right\rangle
$$

where $\langle\cdot, \cdot\rangle$ is the real scalar product. Suppose that $(\mathcal{H}-z) f=0$ and $(\mathcal{H}-z) g=0$. Then

$$
W[f, g]=\text { const. }
$$

Moreover,

$$
\begin{aligned}
& W\left[f_{1}(\cdot, \lambda), f_{2}(\cdot, \lambda)\right]=2 i \lambda, W\left[f_{3}(\cdot, \lambda), \tilde{f}_{4}(\cdot, \lambda)\right]=-2 \mu, \\
& W\left[f_{1}(\cdot, \lambda), f_{3}(\cdot, \lambda)\right]=W\left[f_{2}(\cdot, \lambda), f_{3}(\cdot, \lambda)\right]=0,
\end{aligned}
$$

where $f_{1}, f_{2}, f_{3}, \tilde{f}_{4}$ are as in Lemmas 5.2, 5.3, and 5.5. There exists a unique choice of $c_{1}, c_{2} \in \mathbb{C}$ so that

$$
f_{4}(\cdot, \lambda):=\tilde{f}_{4}(\cdot, \lambda)-c_{1}(\lambda) f_{1}(\cdot, \lambda)-c_{2}(\lambda) f_{2}(\cdot, \lambda)
$$

satisfies

$$
W\left[f_{1}(\cdot, \lambda), f_{4}(\cdot, \lambda)\right]=W\left[f_{2}(\cdot, \lambda), f_{4}(\cdot, \lambda)\right]=0 .
$$

Moreover, $\bar{f}_{4}(\cdot, \lambda)=f_{4}(\cdot,-\lambda)=f_{4}(\cdot, \lambda)$. Finally, we also have $W\left[f_{3}(\cdot, \lambda), f_{4}(\cdot, \lambda)\right]$ $=-2 \mu$ and $c_{j}(\lambda)=O\left(\lambda^{-1}\right)$ for $j=1,2$ as $|\lambda| \rightarrow \infty$.

Proof. Compute

$$
\begin{aligned}
\frac{d}{d x} W[f, g](x) & =\left\langle\sigma_{3} f^{\prime \prime}, \sigma_{3} g\right\rangle-\left\langle\sigma_{3} f, \sigma_{3} g^{\prime \prime}\right\rangle \\
& =\left\langle(1-z) \sigma_{3} f+V f, \sigma_{3} g\right\rangle-\left\langle\sigma_{3} f,(1-z) \sigma_{3} g+V g\right\rangle \\
& =\left\langle\sigma_{3} V f, g\right\rangle-\left\langle V^{*} \sigma_{3} f, g\right\rangle=0 .
\end{aligned}
$$

The statements about the Wronskians follow from the asymptotics in the previous lemmas. Now define

$$
f_{4}(\cdot, \lambda)=\tilde{f}_{4}(\cdot, \lambda)-c_{1}(\lambda) f_{1}(\cdot, \lambda)-c_{2}(\lambda) f_{2}(\cdot, \lambda)
$$

so that

$$
W\left[f_{1}(\cdot, \lambda), f_{4}(\cdot, \lambda)\right]=W\left[f_{2}(\cdot, \lambda), f_{4}(\cdot, \lambda)\right]=0
$$


Thus, we need that

$$
\begin{aligned}
& 0=W\left[f_{1}(\cdot, \lambda), \tilde{f}_{4}(\cdot, \lambda)\right]-c_{2}(\lambda) W\left[f_{1}(\cdot, \lambda), f_{2}(\cdot, \lambda)\right] \\
& 0=W\left[f_{2}(\cdot, \lambda), \tilde{f}_{4}(\cdot, \lambda)\right]-c_{1}(\lambda) W\left[f_{2}(\cdot, \lambda), f_{1}(\cdot, \lambda)\right]
\end{aligned}
$$

and therefore

$$
c_{2}(\lambda)=(2 i \lambda)^{-1} W\left[f_{1}(\cdot, \lambda), \tilde{f}_{4}(\cdot, \lambda)\right], \quad c_{1}(\lambda)=-(2 i \lambda)^{-1} W\left[f_{2}(\cdot, \lambda), \tilde{f}_{4}(\cdot, \lambda)\right] .
$$

It follows from (5.9) and (5.18) that $c_{1}(\lambda)=O\left(\lambda^{-1}\right)$ and $c_{2}(\lambda)=O\left(\lambda^{-1}\right)$. By inspection,

$$
\bar{c}_{1}(\lambda)=(2 i \lambda)^{-1} W\left[\tilde{f}_{4}(\cdot, \lambda), f_{1}(\cdot, \lambda)\right]=c_{2}(\lambda),
$$

which implies that $\bar{f}_{4}(\cdot, \lambda)=f_{4}(\cdot, \lambda)$ by Corollary 5.7. Also,

$$
c_{1}(-\lambda)=(2 i \lambda)^{-1} W\left[f_{2}(\cdot,-\lambda), \tilde{f}_{4}(\cdot,-\lambda)\right]=(2 i \lambda)^{-1} W\left[f_{1}(\cdot, \lambda), \tilde{f}_{4}(\cdot, \lambda)\right]=c_{2}(\lambda),
$$

and by Corollary 5.7 again $f_{4}(\cdot,-\lambda)=f_{4}(\cdot, \lambda)$. Finally, $W\left[f_{3}, f_{4}\right]=W\left[f_{3}, \tilde{f}_{4}\right]=$ $-2 \mu$ as claimed.

We will need the following analogue of (5.18) for $f_{4}$.

Corollary 5.9. Let $f_{4}$ be as in (5.21). Then

$$
\left|\partial_{\lambda}^{\ell} \partial_{x}^{k}\left[e^{-\mu x} f_{4}(x, \lambda)-\left(\begin{array}{l}
0 \\
1
\end{array}\right)\right]\right| \leq C_{k, \ell} \mu^{-1+k} x^{\ell} e^{-\gamma x}
$$

for all $k, \ell \geq 0$.

Proof. This is an immediate consequence of Lemma 5.8 and (5.18).

Recall that we are assuming that $V$ is even. In that case, set

$$
g_{j}(x, \lambda)=f_{j}(-x, \lambda) \text { for } 1 \leq j \leq 4 .
$$

Since $V(x)=V(-x)$, these functions are again solutions of

$$
\mathcal{H} g_{j}(\cdot, \lambda)=\left(1+\lambda^{2}\right) g_{j}(\cdot, \lambda)
$$

which have the same asymptotic behavior as $x \rightarrow-\infty$ as the $f_{j}$ when $x \rightarrow \infty$.

Lemma 5.10. Suppose $F, G$ are $2 \times 2$ matrix solutions of $\mathcal{H} F=z F, \mathcal{H} G=z G$, with some $z \in \mathbb{C}$. Then the matrix Wronskian

$$
\mathcal{W}[F, G](x):=F^{\prime}(x) G(x)-F^{t}(x) G^{\prime}(x)
$$

is independent of $x$. Now suppose that $W[F, F]=0$ or $W[G, G]=0$. Then

$$
\operatorname{det} \mathcal{W}[F, G]=0
$$

iff there exist vectors $a, b \in \mathbb{C}^{2}$, not both zero, such that

$$
F(x) a+G(x) b=0
$$

for all $x \in \mathbb{R}$.

Proof. By assumption, $F=\left[\begin{array}{ll}\phi_{1} & \phi_{2}\end{array}\right], G=\left[\begin{array}{ll}\psi_{1} & \psi_{2}\end{array}\right]$ where $(\mathcal{H}-z) \phi_{j}=0$ and $(\mathcal{H}-z) \psi_{j}=0$ for $j=1,2$. Hence,

$$
\mathcal{W}[F, G](x)=\left(\begin{array}{ll}
W\left[\phi_{1}, \psi_{1}\right](x) & W\left[\phi_{1}, \psi_{2}\right](x) \\
W\left[\phi_{2}, \psi_{1}\right](x) & W\left[\phi_{2}, \psi_{2}\right](x)
\end{array}\right) .
$$


By Lemma 5.8, each of the entries is independent of $x$ and thus $\mathcal{W}[F, G]$. For the second statement, compute

$$
\begin{aligned}
\left(\begin{array}{cc}
\mathcal{W}[F, G] & \mathcal{W}[F, F] \\
\mathcal{W}[G, G] & \mathcal{W}[G, F]
\end{array}\right) & =\left(\begin{array}{ll}
F^{\prime} & -F^{t} \\
G^{\prime t} & -G^{t}
\end{array}\right)\left(\begin{array}{cc}
G & F \\
G^{\prime} & F^{\prime}
\end{array}\right) \\
& =\left(\begin{array}{cc}
0 & I \\
I & 0
\end{array}\right)\left(\begin{array}{cc}
G & F \\
G^{\prime} & F^{\prime}
\end{array}\right)^{t}\left(\begin{array}{cc}
0 & -I \\
I & 0
\end{array}\right)\left(\begin{array}{cc}
G & F \\
G^{\prime} & F^{\prime}
\end{array}\right) .
\end{aligned}
$$

Since $\mathcal{W}[G, F]=-\mathcal{W}[F, G]^{t}$ and $\mathcal{W}[F, F]=0$ (or $\mathcal{W}[G, G]=0$ ) by assumption, we conclude that

$$
[\operatorname{det}(\mathcal{W}[F, G])]^{2}=\left[\operatorname{det}\left(\begin{array}{cc}
G & F \\
G^{\prime} & F^{\prime}
\end{array}\right)\right]^{2} .
$$

Now suppose that there exist vectors $a, b \in \mathbb{C}^{2}$, not both zero, such that

$$
F(x) a+G(x) b=0
$$

for all $x \in \mathbb{R}$. Then, clearly,

$$
\left(\begin{array}{cc}
G(x) & F(x) \\
G^{\prime}(x) & F^{\prime}(x)
\end{array}\right)\left(\begin{array}{l}
b \\
a
\end{array}\right)=0
$$

for every $x \in \mathbb{R}$, and $\operatorname{det} \mathcal{W}[F, G]=0$. Conversely, if $\operatorname{det} \mathcal{W}[F, G]=0$, then for any given $x \in \mathbb{R}$ there exists $\underline{v}=\underline{v}(x) \in \mathbb{C}^{4}$ such that

$$
\left(\begin{array}{cc}
G(x) & F(x) \\
G^{\prime}(x) & F^{\prime}(x)
\end{array}\right) \underline{v}=0 .
$$

Fix $x=0$, say, and let $\underline{v}=\left(\begin{array}{l}b \\ a\end{array}\right)$ with $a, b \in \mathbb{C}^{2}$. Then the column vector $y(x):=$ $G(x) b+F(x) a$ is a solution of $(\mathcal{H}-z) y=0$ with $y(0)=0$ and $y^{\prime}(0)=0$. By the uniqueness theorem, $y(x)=0$ for all $x \in \mathbb{R}$, and we are done.

Definition 5.11. With $f_{j}(\cdot, \lambda), g_{j}(\cdot, \lambda)$ as above, set for each $\lambda \in \mathbb{R}$,

$$
\begin{aligned}
F_{1}(\cdot, \lambda):=\left(f_{1}(\cdot, \lambda), f_{3}(\cdot, \lambda)\right), & F_{2}(\cdot, \lambda)=\left(f_{2}(\cdot, \lambda), f_{4}(\cdot, \lambda)\right), \\
G_{1}(\cdot, \lambda):=\left(g_{2}(\cdot, \lambda), g_{4}(\cdot, \lambda)\right), & G_{2}(\cdot, \lambda)=\left(g_{1}(\cdot, \lambda), g_{3}(\cdot, \lambda)\right) .
\end{aligned}
$$

Remark 5.12. At this point it may be helpful to consider the case $V=0$. Then

$$
\begin{aligned}
F_{1}(x, \lambda) & =\left(\begin{array}{cc}
e^{i x \lambda} & 0 \\
0 & e^{-\mu x}
\end{array}\right), F_{2}(x, \lambda)=\left(\begin{array}{cc}
e^{-i x \lambda} & 0 \\
0 & e^{\mu x}
\end{array}\right), \\
G_{1}(x, \lambda) & =\left(\begin{array}{cc}
e^{i x \lambda} & 0 \\
0 & e^{-\mu x}
\end{array}\right), G_{2}(x, \lambda)=\left(\begin{array}{cc}
e^{-i x \lambda} & 0 \\
0 & e^{\mu x}
\end{array}\right) .
\end{aligned}
$$

Hence, in that case $F_{1}=G_{1}$ and $F_{2}=G_{2}$.

We record some simple but useful symmetry properties of these matrix solutions.

Lemma 5.13. For all $\lambda \in \mathbb{R}$,

$$
\begin{aligned}
G_{1}(x, \lambda) & =F_{2}(-x, \lambda), \quad G_{2}(x, \lambda)=F_{1}(-x, \lambda), \\
\overline{F_{1}(\cdot, \lambda)} & =F_{1}(\cdot,-\lambda), \quad \overline{F_{2}(\cdot, \lambda)}=F_{2}(\cdot,-\lambda), \\
\overline{G_{1}(\cdot, \lambda)} & =G_{1}(\cdot,-\lambda), \quad \overline{G_{2}(\cdot, \lambda)}=G_{2}(\cdot,-\lambda) .
\end{aligned}
$$

Proof. This is an immediate consequence of the definitions, Corollary [5.7, and Lemma 5.8 
Lemma 5.14. For every $\lambda \in \mathbb{R} \backslash\{0\}$ there exist unique constant $2 \times 2$ matrices $A=A(\lambda), B=B(\lambda)$ with complex entries so that

$$
F_{1}(\cdot, \lambda)=G_{1}(\cdot, \lambda) A(\lambda)+G_{2}(\cdot, \lambda) B(\lambda) .
$$

Then $A(-\lambda)=\overline{A(\lambda)}, B(-\lambda)=\overline{B(\lambda)}$, and

$$
\begin{aligned}
G_{2}(\cdot, \lambda) & =F_{2}(\cdot, \lambda) A(\lambda)+F_{1}(\cdot, \lambda) B(\lambda), \\
\mathcal{W}\left[F_{1}(\cdot, \lambda), G_{2}(\cdot, \lambda)\right] & =A(\lambda)^{t}(2 i \lambda p-2 \mu q), \\
\mathcal{W}\left[F_{1}(\cdot, \lambda), G_{1}(\cdot, \lambda)\right] & =-B(\lambda)^{t}(2 i \lambda p-2 \mu q),
\end{aligned}
$$

where $p=\left(\begin{array}{ll}1 & 0 \\ 0 & 0\end{array}\right)$ and $q=\left(\begin{array}{cc}0 & 0 \\ 0 & 1\end{array}\right)$.

Proof. For each $\lambda \neq 0$, the columns of $G_{1}(\cdot, \lambda), G_{2}(\cdot, \lambda)$ form a basis of the kernel of $\mathcal{H}-\left(\lambda^{2}+1\right)$. Hence, the columns of $F_{1}(\cdot, \lambda)$ are linear combinations of these solutions, hence the existence of $A(\lambda), B(\lambda)$. Replacing $x$ with $-x$ in (5.24) implies (5.25). To check (5.26), compute

$$
\begin{aligned}
\mathcal{W}\left[F_{1}, G_{2}\right] & =\left(A^{t}{G_{1}^{\prime}}^{t}+B^{t}{G_{2}^{\prime}}^{t}\right) G_{2}-\left(A^{t} G_{1}^{t}+B^{t} G_{2}^{t}\right) G_{2}^{\prime} \\
& =A^{t}\left({G_{1}^{\prime}}^{t} G_{2}-G_{1}^{t} G_{2}^{\prime}\right)+B^{t}\left({G_{2}^{\prime}}^{t} G_{2}-G_{2}^{t} G_{2}^{\prime}\right) \\
& =-A^{t}\left({F_{2}^{\prime}}^{t} F_{1}-F_{2}^{t} F_{1}^{\prime}\right)-B^{t}\left({F_{1}^{\prime}}^{t} F_{1}-F_{1}^{t} F_{1}^{\prime}\right) \\
& =-A^{t}\left(\begin{array}{ccc}
W\left[f_{2}, f_{1}\right] & W\left[f_{2}, f_{3}\right] \\
W\left[f_{4}, f_{1}\right] & W\left[f_{4}, f_{3}\right]
\end{array}\right)-B^{t}\left(\begin{array}{cc}
0 & W\left[f_{1}, f_{3}\right] \\
W\left[f_{3}, f_{1}\right] & 0
\end{array}\right) \\
& =A^{t}(2 i \lambda p-2 \mu q),
\end{aligned}
$$

where the last line follows from Lemma 5.8 For (5.27), we compute

$$
\begin{aligned}
\mathcal{W}\left[F_{1}, G_{1}\right] & =\left(A^{t}{G_{1}^{\prime}}^{t}+B^{t}{G_{2}^{\prime}}^{t}\right) G_{1}-\left(A^{t} G_{1}^{t}+B^{t} G_{2}^{t}\right) G_{1}^{\prime} \\
& =A^{t}\left({G_{1}^{\prime}}^{t} G_{1}-G_{1}^{t} G_{1}^{\prime}\right)+B^{t}\left(G_{2}^{\prime t} G_{1}-G_{2}^{t} G_{1}^{\prime}\right) \\
& =-A^{t}\left({F_{2}^{\prime}}^{t} F_{2}-F_{2}^{t} F_{2}^{\prime}\right)-B^{t}\left({F_{1}^{\prime}}^{t} F_{2}-F_{1}^{t} F_{2}^{\prime}\right) \\
& =-A^{t}\left(\begin{array}{ccc}
0 & W\left[f_{2}, f_{4}\right] \\
W\left[f_{4}, f_{2}\right] & 0
\end{array}\right)-B^{t}\left(\begin{array}{cc}
W\left[f_{1}, f_{2}\right] & W\left[f_{1}, f_{4}\right] \\
W\left[f_{3}, f_{2}\right] & W\left[f_{3}, f_{4}\right]
\end{array}\right) \\
& =-B^{t}(2 i \lambda p-2 \mu q),
\end{aligned}
$$

where the last line again follows from Lemma 5.8. Finally, by Lemma 5.13 ,

$$
F_{1}(\cdot,-\lambda)=G_{1}(\cdot,-\lambda) A(-\lambda)+G_{2}(\cdot,-\lambda) B(-\lambda)
$$

is the same as

$$
F_{1}(\cdot, \lambda)=G_{1}(\cdot, \lambda) \overline{A(-\lambda)}+G_{2}(\cdot, \lambda) \overline{B(-\lambda)}
$$

so that $A(\lambda)=\overline{A(-\lambda)}, B(\lambda)=\overline{B(-\lambda)}$ for all $\lambda \in \mathbb{R}$.

The following corollary is natural in view of Remark 5.12. Indeed, the limit $|\lambda| \rightarrow \infty$ should correspond to $V \simeq 0$.

Corollary 5.15. $A(\lambda)$ and $B(\lambda)$ are smooth for $\lambda \neq 0$. Furthermore, $\lambda p A(\lambda)$, $q A(\lambda), \lambda p B(\lambda)$, and $q B(\lambda)$ are smooth functions of $\lambda \in \mathbb{R}$. As $|\lambda| \rightarrow \infty$,

$$
A(\lambda)=I+O\left(\lambda^{-1}\right), \quad B(\lambda)=O\left(\lambda^{-1}\right) .
$$


Proof. The regularity statements are immediate from (5.26) and (5.27). By Remark 5.4,

$$
\begin{aligned}
\mathcal{W} & {\left[F_{1}(\cdot, \lambda), G_{2}(\cdot, \lambda)\right] } \\
= & {\left[f_{1}^{\prime}(0, \lambda) f_{3}^{\prime}(0, \lambda)\right]^{t}\left[g_{1}(0, \lambda) g_{3}(0, \lambda)\right]-\left[f_{1}(0, \lambda) f_{3}(0, \lambda)\right]^{t}\left[g_{1}^{\prime}(0, \lambda) g_{3}^{\prime}(0, \lambda)\right] } \\
= & {\left[f_{1}^{\prime}(0, \lambda) f_{3}^{\prime}(0, \lambda)\right]^{t}\left[f_{1}(0, \lambda) f_{3}(0, \lambda)\right]+\left[f_{1}(0, \lambda) f_{3}(0, \lambda)\right]^{t}\left[f_{1}^{\prime}(0, \lambda) f_{3}^{\prime}(0, \lambda)\right] } \\
= & \left(\begin{array}{cc}
i \lambda+O(1) & O(1) \\
O(1) & -\mu+O(1)
\end{array}\right)\left(I+O\left(\lambda^{-1}\right)\right) \\
& +\left(I+O\left(\lambda^{-1}\right)\right)\left(\begin{array}{cc}
i \lambda+O(1) & O(1) \\
O(1) & -\mu+O(1)
\end{array}\right) \\
= & \left(\begin{array}{cc}
2 i \lambda & 0 \\
0 & -2 \mu
\end{array}\right)+O(1)=2 i \lambda p-2 \mu q+O(1) .
\end{aligned}
$$

It now follows from (5.26) that $A^{t}=I+O\left(\lambda^{-1}\right)$ as $|\lambda| \rightarrow \infty$. Similarly, by Remark 5.6 and the property that $c_{j}(\lambda)=O\left(\lambda^{-1}\right)$,

$$
\begin{aligned}
\mathcal{W} & {\left[F_{1}(\cdot, \lambda), G_{1}(\cdot, \lambda)\right] } \\
= & {\left[f_{1}^{\prime}(0, \lambda) f_{3}^{\prime}(0, \lambda)\right]^{t}\left[g_{2}(0, \lambda) g_{4}(0, \lambda)\right]-\left[f_{1}(0, \lambda) f_{3}(0, \lambda)\right]^{t}\left[g_{2}^{\prime}(0, \lambda) g_{4}^{\prime}(0, \lambda)\right] } \\
= & {\left[f_{1}^{\prime}(0, \lambda) f_{3}^{\prime}(0, \lambda)\right]^{t}\left[f_{2}(0, \lambda) f_{4}(0, \lambda)\right]+\left[f_{1}(0, \lambda) f_{3}(0, \lambda)\right]^{t}\left[f_{2}^{\prime}(0, \lambda) f_{4}^{\prime}(0, \lambda)\right] } \\
= & \left(\begin{array}{cc}
i \lambda+O(1) & O(1) \\
O(1) & -\mu+O(1)
\end{array}\right)\left(I+O\left(\lambda^{-1}\right)\right) \\
& +\left(I+O\left(\lambda^{-1}\right)\right)\left(\begin{array}{cc}
-i \lambda+O(1) & O(1) \\
O(1) & \mu+O(1)
\end{array}\right) \\
= & O(1),
\end{aligned}
$$

and the desired bound follows from (5.27).

The following lemma establishes relations between $A$ and $B$ which are analogous to those satisfied by the reflection and transmission coefficients in scalar scattering theory.

Lemma 5.16. For each $\lambda \neq 0$, the matrices $A(\lambda), B(\lambda)$ satisfy the following relations:

$$
-2 i \lambda p=-2 i \lambda A(\lambda)^{*} p A(\lambda)-2 \mu A(\lambda)^{*} q B(\lambda)+2 \mu B(\lambda)^{*} q A(\lambda)+2 i \lambda B(\lambda)^{*} p B(\lambda),
$$

(5.29) $0=A^{t}(2 i \lambda p-2 \mu q) B-B^{t}(2 i \lambda p-2 \mu q) A$,

$$
0=2 \mu A^{*}(\lambda) q-2 i \lambda B^{*}(\lambda) p-2 \mu q A(\lambda)-2 i \lambda p B(\lambda) .
$$

Proof. Compute $\mathcal{W}\left[F_{1}(\cdot, \lambda), \bar{F}_{1}(\cdot, \lambda)\right]$ in two different ways. For the most part, we will suppress $\lambda$ in our notation for the sake of simplicity. Then, on the one hand,

$$
\mathcal{W}\left[\bar{F}_{1}, F_{1}\right]=\left(\begin{array}{ll}
W\left[\bar{f}_{1}, f_{1}\right] & W\left[\bar{f}_{1}, f_{3}\right] \\
W\left[\bar{f}_{3}, f_{1}\right] & W\left[\bar{f}_{3}, f_{3}\right]
\end{array}\right)=\left(\begin{array}{cc}
-2 i \lambda & 0 \\
0 & 0
\end{array}\right)=-2 i \lambda p .
$$

On the other hand,

$\mathcal{W}\left[\bar{F}_{1}, F_{1}\right]=\mathcal{W}\left[\bar{G}_{1} \bar{A}+\bar{G}_{2} \bar{B}, G_{1} A+G_{2} B\right]$

$$
=A^{*} \mathcal{W}\left[\bar{G}_{1}, G_{1}\right] A+A^{*} \mathcal{W}\left[\bar{G}_{1}, G_{2}\right] B+B^{*} \mathcal{W}\left[\bar{G}_{2}, G_{1}\right] A+B^{*} \mathcal{W}\left[\bar{G}_{2}, G_{2}\right] B
$$


Next, we compute each of the matrix Wronskians on the right-hand side of (5.31). Before doing so, we calculate (see (5.21))

$$
W\left[\bar{f}_{4}, f_{4}\right]=W\left[f_{4}, f_{4}\right]=0 .
$$

Then

$$
\begin{aligned}
\mathcal{W}\left[\bar{G}_{1}, G_{1}\right] & =-\mathcal{W}\left[\bar{F}_{2}, F_{2}\right] \\
& =-\left(\begin{array}{cc}
W\left[\bar{f}_{2}, f_{2}\right] & W\left[\bar{f}_{2}, f_{4}\right] \\
W\left[\bar{f}_{4}, f_{2}\right] & W\left[\bar{f}_{4}, f_{4}\right]
\end{array}\right)=-\left(\begin{array}{cc}
W\left[f_{1}, f_{2}\right] & W\left[f_{1}, f_{4}\right] \\
W\left[f_{4}, f_{1}\right] & W\left[\bar{f}_{4}, f_{4}\right]
\end{array}\right) \\
& =-\left(\begin{array}{cc}
2 i \lambda & 0 \\
0 & 0
\end{array}\right)=-2 i \lambda p
\end{aligned}
$$

and

$$
\begin{aligned}
& \mathcal{W}\left[\bar{G}_{1}, G_{2}\right]=-\mathcal{W}\left[\bar{F}_{2}, F_{1}\right] \\
& =-\left(\begin{array}{ll}
W\left[\bar{f}_{2}, f_{1}\right] & W\left[\bar{f}_{2}, f_{3}\right] \\
W\left[\bar{f}_{4}, f_{1}\right] & W\left[\bar{f}_{4}, f_{3}\right]
\end{array}\right)=-\left(\begin{array}{ll}
\frac{W\left[f_{1}, f_{1}\right]}{W\left[f_{4}, f_{2}\right]} & \frac{W\left[f_{1}, f_{3}\right]}{W\left[f_{4}, f_{3}\right]}
\end{array}\right) \\
& =-\left(\begin{array}{cc}
0 & 0 \\
0 & 2 \mu
\end{array}\right)=-2 \mu q \text {. }
\end{aligned}
$$

Thus,

$$
\mathcal{W}\left[\bar{G}_{2}, G_{1}\right]=\overline{\mathcal{W}\left[G_{2}, \bar{G}_{1}\right]}=-\overline{\mathcal{W}\left[\bar{G}_{1}, G_{2}\right]^{t}}=2 \mu q
$$

Finally,

$$
\begin{aligned}
\mathcal{W}\left[\bar{G}_{2}, G_{2}\right] & =-\mathcal{W}\left[\bar{F}_{1}, F_{1}\right] \\
& =-\left(\begin{array}{cc}
W\left[\bar{f}_{1}, f_{1}\right] & W\left[\bar{f}_{1}, f_{3}\right] \\
W\left[\bar{f}_{3}, f_{1}\right] & W\left[\bar{f}_{3}, f_{3}\right]
\end{array}\right)=-\left(\begin{array}{cc}
W\left[f_{2}, f_{1}\right] & W\left[f_{2}, f_{3}\right] \\
W\left[f_{3}, f_{1}\right] & W\left[f_{3}, f_{3}\right]
\end{array}\right) \\
& =\left(\begin{array}{cc}
2 i \lambda & 0 \\
0 & 0
\end{array}\right)=2 i \lambda p .
\end{aligned}
$$

Inserting this into (5.31) yields

$$
-2 i \lambda p=-2 i \lambda A^{*} p A-2 \mu A^{*} q B+2 \mu B^{*} q A+2 i \lambda B^{*} p B,
$$

as claimed. For the second quadratic relation, we compute $\mathcal{W}\left[F_{1}(\cdot, \lambda), F_{1}(\cdot, \lambda)\right]$ in two different ways. On the one hand,

$$
\mathcal{W}\left[F_{1}, F_{1}\right]=\left(\begin{array}{ll}
W\left[f_{1}, f_{1}\right] & W\left[f_{1}, f_{3}\right] \\
W\left[f_{3}, f_{1}\right] & W\left[f_{3}, f_{3}\right]
\end{array}\right)=0 .
$$

On the other hand,

$$
\begin{aligned}
\mathcal{W}\left[F_{1}, F_{1}\right] & =\mathcal{W}\left[G_{1} A+G_{2} B, G_{1} A+G_{2} B\right] \\
& =A^{t} \mathcal{W}\left[G_{1}, G_{1}\right] A+A^{t} \mathcal{W}\left[G_{1}, G_{2}\right] B+B^{t} \mathcal{W}\left[G_{2}, G_{1}\right] A+B^{t} \mathcal{W}\left[G_{2}, G_{2}\right] B \\
& =-A^{t} \mathcal{W}\left[F_{1}, F_{1}\right] A-A^{t} \mathcal{W}\left[F_{1}, F_{2}\right] B-B^{t} \mathcal{W}\left[F_{2}, F_{1}\right] A-B^{t} \mathcal{W}\left[F_{2}, F_{2}\right] B
\end{aligned}
$$

By Lemma 5.8 .

$$
\mathcal{W}\left[F_{2}, F_{2}\right]=\left(\begin{array}{ll}
W\left[f_{2}, f_{2}\right] & W\left[f_{2}, f_{4}\right] \\
W\left[f_{4}, f_{2}\right] & W\left[f_{4}, f_{4}\right]
\end{array}\right)=0 .
$$

By the same lemma,

$$
\mathcal{W}\left[F_{1}, F_{2}\right]=\left(\begin{array}{ll}
W\left[f_{1}, f_{2}\right] & W\left[f_{1}, f_{4}\right] \\
W\left[f_{3}, f_{2}\right] & W\left[f_{3}, f_{4}\right]
\end{array}\right)=2 i \lambda p-2 \mu q,
$$


and therefore,

$$
\mathcal{W}\left[F_{2}, F_{1}\right]=-\mathcal{W}\left[F_{1}, F_{2}\right]^{t}=-2 i \lambda p+2 \mu q
$$

The conclusion is that

$$
0=-A^{t}(2 i \lambda p-2 \mu q) B+B^{t}(2 i \lambda p-2 \mu q) A,
$$

which is (5.29).

Finally, to obtain (5.30), we compute $\mathcal{W}\left[\bar{F}_{1}, G_{2}\right]$ in two different ways: On the one hand,

$$
\begin{aligned}
\mathcal{W}\left[\bar{F}_{1}, G_{2}\right] & =\mathcal{W}\left[\bar{G}_{1} \bar{A}+\bar{G}_{2} \bar{B}, G_{2}\right] \\
& =A^{*} \mathcal{W}\left[\bar{G}_{1}, G_{2}\right]+B^{*} \mathcal{W}\left[\bar{G}_{2}, G_{2}\right] \\
& =-A^{*} \mathcal{W}\left[\bar{F}_{2}, F_{1}\right]-B^{*} \mathcal{W}\left[\bar{F}_{1}, F_{1}\right] \\
& =-2 \mu A^{*} q+2 i \lambda B^{*} p
\end{aligned}
$$

and on the other hand,

$$
\begin{aligned}
\mathcal{W}\left[\bar{F}_{1}, G_{2}\right] & =\mathcal{W}\left[\bar{F}_{1}, F_{2} A+F_{1} B\right] \\
& =\mathcal{W}\left[\bar{F}_{1}, F_{2}\right] A+\mathcal{W}\left[\bar{F}_{1}, F_{1}\right] B \\
& =-2 \mu q A-2 i \lambda p B
\end{aligned}
$$

as claimed.

Next we turn to the important question of invertibility of $A(\lambda)$.

Lemma 5.17. If $\lambda \neq 0$, then the following are equivalent:

- $\operatorname{det} A(\lambda)=0$.

- $E=\lambda^{2}+1$ is an eigenvalue of $\mathcal{H}$.

- $\operatorname{det} \mathcal{W}\left[F_{1}(\cdot, \lambda), G_{2}(\cdot, \lambda)\right]=0$.

Proof. Since $\lambda \neq 0$, the first and third properties are equivalent by (5.26). Now suppose that $\lambda^{2}+1$ is an eigenvalue. Then $F_{1}(\cdot, \lambda)\left(\begin{array}{l}0 \\ 1\end{array}\right)$ and $G_{2}(\cdot, \lambda)\left(\begin{array}{l}0 \\ 1\end{array}\right)$ are linearly dependent (note: the sign of $\lambda$ is irrelevant here). Since

$$
F_{1}(\cdot, \lambda)\left(\begin{array}{l}
0 \\
1
\end{array}\right)=G_{1}(\cdot, \lambda) A(\lambda)\left(\begin{array}{l}
0 \\
1
\end{array}\right)+G_{2}(\cdot, \lambda) B(\lambda)\left(\begin{array}{l}
0 \\
1
\end{array}\right)
$$

we conclude that

$$
A(\lambda)\left(\begin{array}{l}
0 \\
1
\end{array}\right)=0, \quad B(\lambda)\left(\begin{array}{l}
0 \\
1
\end{array}\right)=\left(\begin{array}{l}
0 \\
\alpha
\end{array}\right)
$$

for some $\alpha \neq 0$. In particular, $A(\lambda)$ is singular, as claimed.

Conversely, let $A(\lambda) v=0$ for some $v \in \mathbb{C}^{2}, v \neq 0$. Then (5.28) implies that

$$
\begin{aligned}
-2 i \lambda\langle p v, v\rangle= & -2 i \lambda\left\langle A(\lambda)^{*} p A(\lambda) v, v\right\rangle-2 \mu\left\langle A(\lambda)^{*} q B(\lambda) v, v\right\rangle \\
& +2 \mu\left\langle B(\lambda)^{*} q A(\lambda) v, v\right\rangle+2 i \lambda\left\langle B(\lambda)^{*} p B(\lambda) v, v\right\rangle \\
= & 2 i \lambda\|p B v\|^{2},
\end{aligned}
$$

and hence $-2 i \lambda\|p v\|^{2}=2 i \lambda\|p B v\|^{2}$. Since $\lambda \neq 0$, this implies that $p v=0$ and $p B v=0$. In other words, both $v$ and $B(\lambda) v$ are parallel to $\left(\begin{array}{l}0 \\ 1\end{array}\right)$. By the previous paragraph, this implies that $\lambda^{2}+1$ is an eigenvalue. 
Unlike the scalar case, where rapid decay of the potential insures absence of embedded eigenvalues, this is not true in the system case. Indeed, take $V_{2}=0$ and $V_{1}<0$. If $\left|V_{1}\right|$ is sufficiently large, then there is $E>1$ and $f \in L^{2}(\mathbb{R})$ so that

$$
\left(\partial_{x x}-1-V_{1}\right) f=E f .
$$

This implies that $\mathcal{H}\left(\begin{array}{l}0 \\ f\end{array}\right)=E\left(\begin{array}{l}0 \\ f\end{array}\right)$ so that $E$ becomes an embedded eigenvalue of $\mathcal{H}$.

The case $E=1$ requires more care.

Definition 5.18. We say that $E= \pm 1$ is a resonance of $\mathcal{H}$ provided $\mathcal{H} f= \pm f$ has a solution $f \in L^{\infty} \backslash L^{2}$.

First, we characterize the solutions $f \in L^{\infty} \backslash L^{2}$.

Lemma 5.19. Any solution $f \in L^{\infty} \backslash L^{2}$ of $\mathcal{H} f=f$ is of the form

$$
f(x)=C_{ \pm}\left(\begin{array}{l}
1 \\
0
\end{array}\right)+O\left(e^{\mp \gamma x}\right) \text { as } x \rightarrow \pm \infty
$$

where both $C_{+} \neq 0$ and $C_{-} \neq 0$. Similarly, solutions of $\mathcal{H} f=-f$ are of the form

$$
f(x)=C_{ \pm}\left(\begin{array}{l}
0 \\
1
\end{array}\right)+O\left(e^{\mp \gamma x}\right) \text { as } x \rightarrow \pm \infty .
$$

Proof. In view of Remark 5.4, any such $f$ has to be a linear combination of

$$
f_{1}(\cdot, 0), \partial_{\lambda} f_{1}(\cdot, 0), f_{3}(\cdot, 0), f_{4}(\cdot, 0) .
$$

Clearly, only $f_{1}(\cdot, 0), f_{3}(\cdot, \lambda)$ can occur in this linear combination when $x \rightarrow \infty$, and similarly when $x \rightarrow-\infty$.

There is a characterization similar to Lemma 5.17 for the endpoint $E=1$ ( $E=$ -1 is analogous).

Lemma 5.20. $E=1$ is a resonance or an eigenvalue of $\mathcal{H}$ iff $\operatorname{det} \mathcal{W}\left[F_{1}(\cdot, 0), G_{2}(\cdot, 0)\right]$ vanishes.

Proof. By definition and the proof of Lemma 5.19, $E=1$ is a resonance or an eigenvalue of $\mathcal{H}$ iff there exist $a, b \in \mathbb{C}^{2}$ not zero such that $F_{1}(\cdot, 0) a$ and $G_{2}(\cdot, 0) b$ are linearly dependent. In view of (5.32) and Lemma 5.10 this is in turn equivalent to $\operatorname{det} \mathcal{W}\left[F_{1}(\cdot, 0), G_{2}(\cdot, 0)\right]=0$, as claimed.

Define

$$
D(\lambda):=\mathcal{W}\left[F_{1}(\cdot, \lambda), G_{2}(\cdot, \lambda)\right]
$$

for all $\lambda \in \mathbb{R}$. By (5.26),

$$
\begin{aligned}
(2 i \lambda p-2 \mu q) A(\lambda) & =\mathcal{W}\left[F_{1}(\cdot, \lambda), G_{2}(\cdot, \lambda)\right]^{t}=-\mathcal{W}\left[G_{2}(\cdot, \lambda), F_{1}(\cdot, \lambda)\right] \\
& =\mathcal{W}\left[F_{1}(\cdot, \lambda), G_{2}(\cdot, \lambda)\right]=D(\lambda), \\
D(\lambda)^{t} & =-\mathcal{W}\left[G_{2}(\cdot, \lambda), F_{1}(\cdot, \lambda)\right]=\mathcal{W}\left[F_{1}(\cdot, \lambda), G_{2}(\cdot, \lambda)\right]=D(\lambda), \\
D(-\lambda) & =\mathcal{W}\left[F_{1}(\cdot,-\lambda), G_{2}(\cdot,-\lambda)\right]=\overline{\mathcal{W}\left[F_{1}(\cdot, \lambda), G_{2}(\cdot, \lambda)\right]}=\overline{D(\lambda)}
\end{aligned}
$$

for all $\lambda \in \mathbb{R}$. Combining the previous two lemmas therefore yields

Corollary 5.21. The following properties are equivalent:

- There are no eigenvalues in $[1, \infty)$ and $E=1$ is not a resonance.

- $D(\lambda)$ is invertible for all $\lambda \in \mathbb{R}$. 
In that case,

$$
A(\lambda)^{-1}=D(\lambda)^{-1}(2 i \lambda p-2 \mu q)
$$

for all $\lambda \in \mathbb{R}$.

The point of the final statement is that it should be viewed as a definition of the left-hand side in case $\lambda=0$. Note that (5.27) allows us to conclude that

$$
\lim _{\lambda \rightarrow 0}(2 i \lambda p B(\lambda)-2 q B(\lambda))=\mathcal{W}\left[F_{2}(\cdot, 0), G_{2}(\cdot, 0)\right] .
$$

6. Scattering SOlutions, the Resolvent, AND THE DISTORTED FOURIER TRANSFORM

From now on, we shall assume that the conditions of Corollary 5.21 hold. We will call such Hamiltonians admissible.

Definition 6.1. We say that $\mathcal{H}$ is admissible if it satisfies the requirements of Definition 5.1, if there are no eigenvalues in the essential spectrum $(-\infty,-1] \cup[1, \infty)$ and if the edges \pm 1 are not resonances.

Later we will prove that the linearization of NLS around a ground state is admissible. It turns out that this class of $\mathcal{H}$ admits the construction of scattering solutions for all energies $|E|>1$; see Lemma 6.3. We start with a rather obvious lemma about the smoothness of $D(\lambda)^{-1}$.

Lemma 6.2. Let $\mathcal{H}$ be admissible. Then both $D(\lambda)$ and $D^{-1}(\lambda)$ are smooth functions in $\lambda \in \mathbb{R}$. Moreover, $D(\lambda)^{-1} \lambda=O(1)$ as $|\lambda| \rightarrow \infty$.

Proof. Since both $F_{1}(x, \lambda)$ and $G_{2}(x, \lambda)$ are smooth functions in $\lambda$, it follows from (5.33) that $D(\lambda)$ and therefore also $\operatorname{det}(D(\lambda))$ are smooth. Since Corollary 5.21 implies that $\operatorname{det}(D(\lambda)) \neq 0$ for all $\lambda \in \mathbb{R}$, we conclude from Cramer's rule that $D^{-1}(\lambda)$ is smooth for all $\lambda$. Finally, the asymptotics of $D^{-1}(\lambda)$ follows from Corollary 5.15 .

For the remainder of this section, admissibility of $\mathcal{H}$ will be a standing assumption and we will not mention it further.

Lemma 6.3. Let $\underline{e}=\left(\begin{array}{l}1 \\ 0\end{array}\right)$. Then for all $\lambda \in \mathbb{R}$

$$
\begin{aligned}
\mathcal{F}(x, \lambda) & :=2 i \lambda F_{1}(x, \lambda) D(\lambda)^{-1} \underline{e}, \\
\mathcal{G}(x, \lambda) & :=2 i \lambda G_{2}(x, \lambda) D(\lambda)^{-1} \underline{e}
\end{aligned}
$$

are bounded solutions of $\mathcal{H} f=\left(1+\lambda^{2}\right) f$. Moreover, their asymptotics are given $b y{ }^{21}$

$$
\begin{aligned}
& \mathcal{F}(x, \lambda)=s(\lambda)\left[e^{i x \lambda} \underline{e}+O\left((1+|\lambda|)^{-1} e^{-\gamma x}\right)\right]+O\left(\lambda(1+|\lambda|)^{-2} e^{-\mu x}\right) \quad \text { as } x \rightarrow \infty, \\
& \mathcal{F}(x, \lambda)=\left[e^{i x \lambda}+r(\lambda) e^{-i x \lambda}\right] \underline{e}+O\left(\lambda(1+|\lambda|)^{-2} e^{\gamma x}\right) \quad \text { as } x \rightarrow-\infty, \\
& \mathcal{G}(x, \lambda)=s(\lambda)\left[e^{-i x \lambda} \underline{e}+O\left((1+|\lambda|)^{-1} e^{\gamma x}\right)\right]+O\left(\lambda(1+|\lambda|)^{-2} e^{\mu x}\right) \quad \text { as } x \rightarrow-\infty, \\
& \mathcal{G}(x, \lambda)=\left[e^{-i x \lambda}+r(\lambda) e^{i x \lambda}\right] \underline{e}+O\left(\lambda(1+|\lambda|)^{-2} e^{-\gamma x}\right) \quad \text { as } x \rightarrow \infty,
\end{aligned}
$$

where

$$
s(\lambda) \underline{e}=2 i \lambda p D(\lambda)^{-1} \underline{e} \text { and } r(\lambda) \underline{e}=2 i \lambda p B(\lambda) D(\lambda)^{-1} \underline{e}
$$

\footnotetext{
${ }^{21}$ Here $O\left(\lambda(1+|\lambda|)^{-2}\right)$ stands for a function which vanishes linearly as $\lambda \rightarrow 0$ and decays like $\lambda^{-1}$ as $\lambda \rightarrow \infty$.
} 
are smooth functions for all $\lambda \in \mathbb{R}$ with $s(0)=0, r(0)=-1$. The matrix $S(\lambda):=$ $\left(\begin{array}{ll}s(\lambda) & r(\lambda) \\ r(\lambda) & s(\lambda)\end{array}\right)$ is unitary. In fact, one has

$$
S(\lambda)^{*}=S(\lambda)^{-1}=S(-\lambda)
$$

for all $\lambda \in \mathbb{R}$.

Proof. By Lemmas 5.3 and 5.2 ,

$$
\begin{aligned}
\mathcal{F}(x, \lambda) & =2 i \lambda F_{1}(x, \lambda) p D(\lambda)^{-1} \underline{e}+2 i \lambda F_{1}(x, \lambda) q D(\lambda)^{-1} \underline{e} \\
& =s(\lambda) f_{1}(x, \lambda)+O\left(\lambda(1+|\lambda|)^{-1} e^{-\mu x}\right) \\
& =s(\lambda)\left[e^{i x \lambda} \underline{e}+O\left((1+|\lambda|)^{-1} e^{-\gamma x}\right)\right]+O\left(\lambda(1+|\lambda|)^{-1} e^{-\mu x}\right)
\end{aligned}
$$

as $x \rightarrow \infty$. On the other hand, as $x \rightarrow-\infty$,

$$
\begin{aligned}
\mathcal{F}(x, \lambda) & =G_{1}(x, \lambda) \underline{e}+G_{2}(x, \lambda) B(\lambda) A(\lambda)^{-1} \underline{e} \\
& =f_{2}(-x, \lambda)+G_{2}(x, \lambda) p B(\lambda) A(\lambda)^{-1} \underline{e}+G_{2}(x, \lambda) q B(\lambda) A(\lambda)^{-1} \underline{e} \\
& =f_{2}(-x, \lambda)+r(\lambda) f_{1}(-x, \lambda)+O\left(\lambda(1+|\lambda|)^{-1} e^{\gamma x}\right) \\
& =\left[e^{i x \lambda}+r(\lambda) e^{-i x \lambda}\right] \underline{e}+O\left(\lambda(1+|\lambda|)^{-1} e^{\gamma x}\right) .
\end{aligned}
$$

Here we used that $\lambda^{-1} q B(\lambda) A(\lambda)^{-1} \underline{e}=2 i B(\lambda) D^{-1}(\lambda) \underline{e}$ is smooth in $\lambda$. The asymptotics for $\mathcal{G}$ now follow since $\mathcal{G}(x, \lambda)=\mathcal{F}(-x, \lambda)$.

As far as unitarity is concerned, (5.26) implies that for $\lambda \neq 0$,

$$
\begin{aligned}
& -2 i \lambda\left\langle p A^{-1}(\lambda) \underline{e}, A^{-1}(\lambda) \underline{e}\right\rangle \\
& =-2 i \lambda\left\langle A(\lambda)^{*} p A(\lambda) A(\lambda)^{-1} \underline{e}, A(\lambda)^{-1} \underline{e}\right\rangle-2 \mu\left\langle A(\lambda)^{*} q B(\lambda) A(\lambda)^{-1} \underline{e}, A(\lambda)^{-1} \underline{e}\right\rangle \\
& +2 \mu\left\langle B(\lambda)^{*} q A(\lambda) A(\lambda)^{-1} \underline{e}, A(\lambda)^{-1} \underline{e}\right\rangle+2 i \lambda\left\langle B(\lambda)^{*} p B(\lambda) A(\lambda)^{-1} \underline{e}, A(\lambda)^{-1} \underline{e}\right\rangle .
\end{aligned}
$$

Since $s(\lambda) \underline{e}=p A(\lambda)^{-1} \underline{e}$ and $r(\lambda) \underline{e}=B(\lambda) A(\lambda)^{-1} \underline{e}$ for $\lambda \neq 0$, we obtain from this that

$$
|s(\lambda)|^{2}+|r(\lambda)|^{2}=1,
$$

which also extends to $\lambda=0$ by continuity. On the other hand, (5.30) implies that

$$
\begin{aligned}
0 & =2 \mu\left\langle A^{*}(\lambda) q A(\lambda)^{-1} \underline{e}, A(\lambda)^{-1} \underline{e}\right\rangle-2 i \lambda\left\langle B^{*}(\lambda) p A(\lambda)^{-1} \underline{e}, A(\lambda)^{-1} \underline{e}\right\rangle \\
& -2 \mu\left\langle q A(\lambda) A(\lambda)^{-1} \underline{e}, A(\lambda)^{-1} \underline{e}\right\rangle-2 i \lambda\left\langle p B(\lambda) A(\lambda)^{-1} \underline{e}, A(\lambda)^{-1} \underline{e}\right\rangle \\
& =2 \mu\left\langle q A(\lambda)^{-1} \underline{e}, \underline{e}\right\rangle-2 i \lambda\left\langle p A(\lambda)^{-1} \underline{e}, p B(\lambda) A(\lambda)^{-1} \underline{e}\right\rangle \\
& -2 \mu\left\langle q \underline{e}, A(\lambda)^{-1} \underline{e}\right\rangle-2 i \lambda\left\langle p B(\lambda) A(\lambda)^{-1} \underline{e}, p A(\lambda)^{-1} \underline{e}\right\rangle \\
& =-2 i \lambda s(\lambda) \bar{r}(\lambda)-2 i \lambda r(\lambda) \bar{s}(\lambda)
\end{aligned}
$$

so that $0=s(\lambda) \bar{r}(\lambda)+r(\lambda) \bar{s}(\lambda)$, as claimed. Finally,

$$
\begin{aligned}
& s(-\lambda) \underline{e}=-2 i \lambda p D(-\lambda)^{-1} \underline{e}=\overline{2 i \lambda D(\lambda)^{-1} \underline{e}}=\overline{s(\lambda)} \underline{e}, \\
& r(-\lambda) \underline{e}=-2 i \lambda p B(-\lambda) D(-\lambda)^{-1} \underline{e}=\overline{2 i \lambda B(\lambda) D(\lambda)^{-1} \underline{e}}=\overline{r(\lambda)} \underline{e}
\end{aligned}
$$

for all $\lambda \in \mathbb{R}$ which proves that $S(-\lambda)=\overline{S(\lambda)}^{t}=S(\lambda)^{*}$.

The solutions $\mathcal{F}(\cdot, \lambda), \mathcal{G}(\cdot, \lambda)$ are fundamental for several reasons, one being that they form the (distorted) Fourier basis associated with $\mathcal{H}$. This will be clarified later. First, we show that any globally bounded solution for $\lambda \neq 0$ is a linear combination of these two solutions. 
Lemma 6.4. Any solution $f$ of $\mathcal{H} f=\left(\lambda^{2}+1\right) f$ with $\lambda \neq 0$ and $f \in L^{\infty}(\mathbb{R})$ is a linear combination of $\mathcal{F}(\cdot, \lambda)$ and $\mathcal{G}(\cdot, \lambda)$.

Proof. By (the proof of) Lemma 5.10, see (5.23), the matrix

$$
\left(\begin{array}{ll}
F_{1}(x, \lambda) & G_{2}(x, \lambda) \\
F_{1}^{\prime}(x, \lambda) & G_{2}^{\prime}(x, \lambda)
\end{array}\right)
$$

is invertible for all $x \in \mathbb{R}$. As noted in the proof of that lemma, this means that the four columns of $F_{1}, G_{2}$ are linearly independent. Hence, there exist $\underline{v}, \underline{w} \in \mathbb{C}^{2}$ so that

$$
f(x)=F_{1}(x, \lambda) \underline{v}+G_{2}(x, \lambda) \underline{w}
$$

for all $x \in \mathbb{R}$. As $x \rightarrow-\infty$,

$$
f(x)=G_{1}(x, \lambda) A(\lambda) \underline{v}+G_{2}(x, \lambda)[B(\lambda) \underline{v}+\underline{w}]
$$

remains bounded iff $A(\lambda) \underline{v}$ is parallel to $\underline{e}$. Similarly, as $x \rightarrow \infty$,

$$
f(x)=F_{1}(x, \lambda)[\underline{v}+B(\lambda) \underline{w}]+F_{2}(x, \lambda) A(\lambda) \underline{w}
$$

remains bounded iff $A(\lambda) \underline{w}$ is parallel to $\underline{e}$. Hence,

$$
\underline{v}=\alpha A^{-1}(\lambda) \underline{e}, \quad \underline{w}=\beta A^{-1}(\lambda) \underline{w}
$$

for some constants $\alpha, \beta \in \mathbb{C}$. This implies that

$$
f(x)=\alpha \mathcal{F}(x, \lambda)+\beta \mathcal{G}(x, \lambda)
$$

for all $x \in \mathbb{R}$, as desired.

We can now obtain expressions for the resolvent kernel on the essential spectrum.

Lemma 6.5. For all $\lambda \geq 0$,

$$
\begin{aligned}
& \left(\mathcal{H}-\left(\lambda^{2}+1+i 0\right)\right)^{-1}(x, y)= \begin{cases}-F_{1}(x, \lambda) D^{-1}(\lambda) G_{2}^{t}(y, \lambda) \sigma_{3} & \text { if } x \geq y, \\
-G_{2}(x, \lambda) D^{-1}(\lambda) F_{1}^{t}(y, \lambda) \sigma_{3} & \text { if } x \leq y,\end{cases} \\
& \left(\mathcal{H}-\left(\lambda^{2}+1-i 0\right)\right)^{-1}(x, y)= \begin{cases}-F_{1}(x,-\lambda) D^{-1}(-\lambda) G_{2}^{t}(y,-\lambda) \sigma_{3} & \text { if } x \geq y, \\
-G_{2}(x,-\lambda) D^{-1}(-\lambda) F_{1}^{t}(y,-\lambda) \sigma_{3} & \text { if } x \leq y .\end{cases}
\end{aligned}
$$

Proof. There exist matrices $\mathcal{M}_{1}(y, \lambda)$ and $\mathcal{M}_{2}(y, \lambda)$ so that

$$
\left(\mathcal{H}-\left(\lambda^{2}+1+i 0\right)\right)^{-1}(x, y)= \begin{cases}F_{1}(x, \lambda) \mathcal{M}_{1}(y, \lambda) & \text { if } x \geq y \\ G_{2}(x, \lambda) \mathcal{M}_{2}(y, \lambda) & \text { if } x \leq y\end{cases}
$$

The choice of $F_{1}(x, \lambda)$ and $G_{2}(x, \lambda)$ for $\lambda \geq 0$ is due to the fact that these are the only functions that remain bounded for $\lambda+i \epsilon$ as $x \rightarrow \infty$ or $x \rightarrow-\infty$, respectively. As usual, one needs compatibility conditions at $x=y$. Here they take the form

$$
\begin{aligned}
& F_{1}(x, \lambda) \mathcal{M}_{1}(x, \lambda)-G_{2}(x, \lambda) \mathcal{M}_{2}(x, \lambda)=0, \\
& F_{1}^{\prime}(x, \lambda) \mathcal{M}_{1}(x, \lambda)-G_{2}^{\prime}(x, \lambda) \mathcal{M}_{2}(x, \lambda)=-\sigma_{3} .
\end{aligned}
$$

To see why, observe that for any Schwartz function, say, $h(x)=\left(\begin{array}{l}h_{1}(x) \\ h_{2}(x)\end{array}\right)$ we need to ensure that

$$
f(x):=G_{2}(x, \lambda) \int_{x}^{\infty} \mathcal{M}_{2}(y, \lambda) h(y) d y+F_{1}(x, \lambda) \int_{-\infty}^{x} \mathcal{M}_{1}(y, \lambda) h(y) d y
$$

satisfies

$$
\left(\mathcal{H}-\left(\lambda^{2}+1\right)\right) f=\left(\sigma_{3}\left(-\partial_{x x}+1\right)+V\right) f(x)=h(x)
$$


for all $x \in \mathbb{R}$. Direct differentiation leads to the conditions above. In matrix notation,

$$
\left(\begin{array}{ll}
F_{1}(x, \lambda) & G_{2}(x, \lambda) \\
F_{1}^{\prime}(x, \lambda) & G_{2}^{\prime}(x, \lambda)
\end{array}\right)\left(\begin{array}{c}
\mathcal{M}_{1}(x, \lambda) \\
-\mathcal{M}_{2}(x, \lambda)
\end{array}\right)=\left(\begin{array}{c}
0 \\
-\sigma_{3}
\end{array}\right)
$$

By Lemma 5.10 (or more precisely, its proof), the $4 \times 4$ matrix on the left-hand side is invertible for all $x \in \mathbb{R}$. In fact, in view of (5.22) and (5.32) we have the following explicit expression for the inverse:

$$
\begin{aligned}
\left(\begin{array}{ll}
F_{1} & G_{2} \\
F_{1}^{\prime} & G_{2}^{\prime}
\end{array}\right)^{-1} & =\left(\begin{array}{cc}
-D^{-t} & 0 \\
0 & D^{-1}
\end{array}\right)\left(\begin{array}{cc}
0 & I \\
I & 0
\end{array}\right)\left(\begin{array}{cc}
F_{1}^{t} & F_{1}^{\prime t} \\
G_{2}^{t} & G_{2}^{\prime t}
\end{array}\right)\left(\begin{array}{cc}
0 & -I \\
I & 0
\end{array}\right) \\
& =\left(\begin{array}{cc}
-D^{-t} G_{2}^{\prime t} & D^{-t} G_{2}^{t} \\
D^{-1} F_{1}^{\prime t} & -D^{-1} F_{1}^{t}
\end{array}\right) .
\end{aligned}
$$

Consequently,

$$
\left(\begin{array}{l}
\mathcal{M}_{1}(x, \lambda) \\
\mathcal{M}_{2}(x, \lambda)
\end{array}\right)=\left(\begin{array}{l}
-D^{-t}(\lambda) G_{2}^{t}(x, \lambda) \sigma_{3} \\
-D^{-1}(\lambda) F_{1}^{t}(x, \lambda) \sigma_{3}
\end{array}\right)=\left(\begin{array}{l}
-D^{-1}(\lambda) G_{2}^{t}(x, \lambda) \sigma_{3} \\
-D^{-1}(\lambda) F_{1}^{t}(x, \lambda) \sigma_{3}
\end{array}\right),
$$

as claimed. The case of $-i 0$ is basically identical, and we are done.

Remark 6.6. If $V=0$, then it is easy to check that Lemma 6.5 yields

$$
\left(\mathcal{H}-\left(\lambda^{2}+1 \pm i 0\right)\right)^{-1}(x, y)=\left(\begin{array}{cc}
\mp \frac{e^{ \pm i \lambda|x-y|}}{2 i \lambda} & 0 \\
0 & -\frac{e^{-\mu|x-y|}}{2 \mu}
\end{array}\right),
$$

which is also an immediate consequence of the standard formulas for the onedimensional scalar free resolvent.

Next, we need to express the jump of the resolvent across the spectrum $[1, \infty)$.

Lemma 6.7. Let

$$
\mathcal{E}(x, \lambda):=[\mathcal{F}(x, \lambda) \mathcal{G}(x, \lambda)]
$$

for all $\lambda \in \mathbb{R}$. Then

$$
\left(\mathcal{H}-\left(\lambda^{2}+1+i 0\right)\right)^{-1}(x, y)-\left(\mathcal{H}-\left(\lambda^{2}+1-i 0\right)\right)^{-1}(x, y)=\frac{-1}{2 i \lambda} \mathcal{E}(x, \lambda) \mathcal{E}^{*}(y, \lambda) \sigma_{3}
$$

for all $\lambda \geq 0$.

Proof. Let $\lambda \geq 0$ and set

$$
\mathcal{S}(x, y ; \lambda):=\left(\mathcal{H}-\left(\lambda^{2}+1+i 0\right)\right)^{-1}(x, y)-\left(\mathcal{H}-\left(\lambda^{2}+1-i 0\right)\right)^{-1}(x, y) .
$$

For fixed $y \in \mathbb{R}$

so that

$$
\left(\mathcal{H}-\left(\lambda^{2}+1\right)\right) \mathcal{S}(\cdot, y ; \lambda)=0
$$

$$
x \mapsto \mathcal{S}(x, y ; \lambda)
$$

is a globally bounded solution. It vanishes identically for $\lambda=0$. If $\lambda>0$, then Lemma 6.4 implies that

$$
\mathcal{S}(x, y ; \lambda)=\mathcal{E}(x ; \lambda) \mathcal{M}(y ; \lambda)
$$

for some matrix $\mathcal{M}(y ; \lambda)$. Lemma 6.5 implies that

$$
\sigma_{3} \overline{\mathcal{S}(x, y ; \lambda)}^{t} \sigma_{3}=-\mathcal{S}(x, y ; \lambda)
$$

Hence,

$$
\sigma_{3} \mathcal{M}(y ; \lambda)^{*} \mathcal{E}(x ; \lambda)^{*} \sigma_{3}=-\mathcal{E}(y ; \lambda) \mathcal{M}(x ; \lambda) .
$$


Since $\mathcal{E}(x, \lambda)$ is invertible for $\lambda>0$ and every $x \in \mathbb{R}$, we conclude that

$$
\mathcal{E}(y ; \lambda)^{-1} \sigma_{3} \mathcal{M}(y ; \lambda)^{*}=-\mathcal{M}(x ; \lambda) \sigma_{3} \mathcal{E}(x ; \lambda)^{-*}=: C(\lambda)
$$

is a matrix which depends only on $\lambda$. Moreover, we see that $C(\lambda)^{*}=-C(\lambda)$. Consequently,

$$
\mathcal{S}(x, y ; \lambda)=-\mathcal{E}(x ; \lambda) C(\lambda) \mathcal{E}(y ; \lambda)^{*} \sigma_{3} .
$$

To determine $C(\lambda)$, we invoke the asymptotics of both sides of this equation as $x \rightarrow \infty$ and $y \rightarrow-\infty$. In view of Lemma 6.5 the left-hand side satisfies

$$
\begin{aligned}
\mathcal{S}(x, y ; \lambda)= & -\left(\begin{array}{cc}
e^{i x \lambda} & 0 \\
0 & 0
\end{array}\right) D^{-1}(\lambda)\left(\begin{array}{cc}
e^{-i y \lambda} & 0 \\
0 & 0
\end{array}\right) \sigma_{3} \\
& +\left(\begin{array}{cc}
e^{-i x \lambda} & 0 \\
0 & 0
\end{array}\right) D^{-1}(-\lambda)\left(\begin{array}{cc}
e^{i y \lambda} & 0 \\
0 & 0
\end{array}\right) \sigma_{3}+o(1) \\
= & -e^{i(x-y) \lambda} \frac{s(\lambda)}{2 i \lambda} p-e^{-i(x-y) \lambda} \frac{s(-\lambda)}{2 i \lambda} p+o(1)
\end{aligned}
$$

in this limit. The matrix $C(\lambda)$ can be written as

$$
C(\lambda)=\left(\begin{array}{cc}
i \alpha & z \\
-\bar{z} & i \beta
\end{array}\right)
$$

where $\alpha, \beta \in \mathbb{R}$ and $z \in \mathbb{C}$. By Lemma 6.3 and (6.4),

$$
\begin{aligned}
& \mathcal{S}(x, y ; \lambda) \\
& =-\left(\begin{array}{cc}
s(\lambda) e^{i x \lambda} & e^{-i x \lambda}+r(\lambda) e^{i x \lambda} \\
0 & 0
\end{array}\right) C(\lambda)\left(\begin{array}{cc}
e^{-i y \lambda}+\bar{r}(\lambda) e^{i y \lambda} & 0 \\
\bar{s}(\lambda) e^{i y \lambda}
\end{array}\right) \sigma_{3}+o(1) \\
& =-\left[e^{i(x+y) \lambda}\left(i \alpha s(\lambda) \bar{r}(\lambda)+z|s(\lambda)|^{2}+i \beta r(\lambda) \bar{s}(\lambda)-\bar{z}|r(\lambda)|^{2}\right)\right.
\end{aligned}
$$

$$
\left.+e^{i(x-y) \lambda}(i \alpha s(\lambda)-r(\lambda) \bar{z})+e^{-i(x-y) \lambda}(-\bar{z} \bar{r}(\lambda)+i \beta \bar{s}(\lambda))-\bar{z} e^{-i(x+y) \lambda}\right] p+o(1) .
$$

Comparing (6.5) with (6.6) yields $z=0$ and

$$
i \alpha s(\lambda)=\frac{s(\lambda)}{2 i \lambda}, \quad i \beta \bar{s}(\lambda)=\frac{s(-\lambda)}{2 i \lambda}=\frac{\bar{s}(\lambda)}{2 i \lambda} .
$$

This implies that $\alpha=\beta=-\frac{1}{2 \lambda}$ or

$$
C(\lambda)=\frac{1}{2 i \lambda} I
$$

and the lemma follows.

Let $P_{d}$ be the Riesz projection onto the discrete spectrum, i.e.,

$$
P_{d}=\frac{1}{2 \pi i} \oint_{\gamma}(z I-\mathcal{H})^{-1} d z
$$

where $\gamma$ is a simple closed curve that encloses the entire discrete spectrum of $\mathcal{H}$ and lies within the resolvent set. Moreover, define $P_{s}=I-P_{d}$ (" $s$ " here stands for "stable").

We now recall a general representation formula for the expression $\left\langle e^{i t \mathcal{H}} P_{s} \phi, \psi\right\rangle$ from [14. It is elementary and was probably known before. Although [14 dealt with dimension three, these particular statements are independent of the dimension 
(basically, (6.8) follows from the Hille-Yoshida theorem). Nevertheless, we present the proof for the reader's convenience.

Lemma 6.8. Assume that $\mathcal{H}$ is admissible. Then there is the representation

$$
e^{i t \mathcal{H}}=\frac{1}{2 \pi i} \int_{|\lambda| \geq 1} e^{i t \lambda}\left[(\mathcal{H}-(\lambda+i 0))^{-1}-(\mathcal{H}-(\lambda-i 0))^{-1}\right] d \lambda+\sum_{j} e^{i t \mathcal{H}} P_{\zeta_{j}}
$$

where the sum runs over the entire discrete spectrum $\left\{\zeta_{j}\right\}_{j}$ and $P_{\zeta_{j}}$ is the Riesz projection corresponding to the eigenvalue $\zeta_{j}$. The formula (6.8) and the convergence of the integral are to be understood in the following weak sense: If $\phi, \psi \in \mathcal{S}$, then

$$
\begin{aligned}
\left\langle e^{i t \mathcal{H}} \phi, \psi\right\rangle= & \lim _{R \rightarrow \infty} \frac{1}{2 \pi i} \int_{R \geq|\lambda| \geq 1} e^{i t \lambda}\left\langle\left[(\mathcal{H}-(\lambda+i 0))^{-1}-(\mathcal{H}-(\lambda-i 0))^{-1}\right] \phi, \psi\right\rangle d \lambda \\
& +\sum_{j}\left\langle e^{i t \mathcal{H}} P_{\zeta_{j}} \phi, \psi\right\rangle
\end{aligned}
$$

for all $t$. The integrand here is well defined in view of Lemma 6.7 .

Proof. We start by checking the following limiting absorption principle:

$$
\sup _{|\lambda| \geq \lambda_{0}, \varepsilon>0}|\lambda|^{\frac{1}{2}}\left\|(\mathcal{H}-(\lambda \pm i \varepsilon))^{-1}\right\|<\infty
$$

where the norm is that of $L^{2, \sigma}(\mathbb{R}) \times L^{2, \sigma}(\mathbb{R}) \rightarrow L^{2,-\sigma}(\mathbb{R}) \times L^{2,-\sigma}(\mathbb{R})$ where $\sigma>\frac{1}{2}$, say, and $\lambda_{0}$ is large. In the free case (i.e., $V=0$ and $\mathcal{H}=\mathcal{H}_{0}$ ) this bound is an immediate consequence of the explicit form of the resolvent. If $V \neq 0$, then we write

$$
(\mathcal{H}-(\lambda \pm i 0))^{-1}=\left(\mathcal{H}_{0}-(\lambda \pm i 0)\right)^{-1}\left(I+V\left(\mathcal{H}_{0}-(\lambda \pm i 0)\right)^{-1}\right)^{-1} .
$$

This is to be understood as an identity between operators $L^{2, \sigma}(\mathbb{R}) \times L^{2, \sigma}(\mathbb{R}) \rightarrow$ $L^{2,-\sigma}(\mathbb{R}) \times L^{2,-\sigma}(\mathbb{R})$ where $\sigma>\frac{1}{2}$. Note that the inverse

$$
\left(I+V\left(\mathcal{H}_{0}-(\lambda \pm i 0)\right)^{-1}\right)^{-1}
$$

exists as an operator on $L^{2, \sigma}(\mathbb{R}) \times L^{2, \sigma}(\mathbb{R}) \rightarrow L^{2, \sigma}(\mathbb{R}) \times L^{2, \sigma}(\mathbb{R})$ because

$$
\sup _{|\lambda| \geq \lambda_{0}}\left\|V\left(\mathcal{H}_{0}-(\lambda \pm i 0)\right)^{-1}\right\|<\frac{1}{2}
$$

for $\lambda_{0}$ large. Hence, (6.9) holds.

The evolution $e^{i t \mathcal{H}}$ is defined via the Hille-Yoshida theorem. Indeed, let $a>0$ be large. Then $i \mathcal{H}-a$ satisfies (with $\rho$ the resolvent set)

$$
\rho(i \mathcal{H}-a) \supset(0, \infty) \text { and }\left\|(i \mathcal{H}-a-\lambda)^{-1}\right\|_{2 \rightarrow 2} \leq \lambda^{-1} \text { for all } \lambda>0 .
$$

The estimate here follows from

$$
\begin{aligned}
\left\|(i \mathcal{H}-a-\lambda)^{-1}\right\| & \leq\left\|\left(i \mathcal{H}_{0}-a-\lambda\right)^{-1}\right\|\left\|\left(I+i V\left(i \mathcal{H}_{0}-a-\lambda\right)^{-1}\right)^{-1}\right\| \\
& \leq(\lambda+a)^{-1} \frac{1}{1-C(a+\lambda)^{-1}}=\frac{1}{\lambda+a-C} \leq \lambda^{-1}
\end{aligned}
$$

provided $a$ is large. Hence $\left\{e^{t(i \mathcal{H}-a)}\right\}_{t \geq 0}$ is a contractive semigroup, so that $\left\|e^{i t \mathcal{H}}\right\|_{2 \rightarrow 2} \leq e^{|t| a}$ for all $t \in \mathbb{R}$. If $\Re z>a$, then there is the Laplace transform

$$
(i \mathcal{H}-z)^{-1}=-\int_{0}^{\infty} e^{-t z} e^{i t \mathcal{H}} d t
$$


as well as its inverse (with $b>a$ and $t>0$ )

$$
e^{i t \mathcal{H}}=-\frac{1}{2 \pi i} \int_{b-i \infty}^{b+i \infty} e^{t z}(i \mathcal{H}-z)^{-1} d z .
$$

While (6.10) converges in the norm sense, defining (6.11) requires more care. The claim is that for any $\phi, \psi \in \operatorname{Dom}(\mathcal{H})=W^{2,2} \times W^{2,2}$,

$$
\left\langle e^{i t \mathcal{H}} \phi, \psi\right\rangle=-\lim _{R \rightarrow \infty} \frac{1}{2 \pi i} \int_{b-i R}^{b+i R} e^{t z}\left\langle(i \mathcal{H}-z)^{-1} \phi, \psi\right\rangle d z .
$$

To verify this, let $t>0$ and use (6.10) to conclude that

$$
\begin{aligned}
-\frac{1}{2 \pi i} \int_{b-i R}^{b+i R} e^{t z}\left\langle(i \mathcal{H}-z)^{-1} \phi, \psi\right\rangle d z & =\frac{1}{2 \pi i} \int_{b-i R}^{b+i R} e^{t z} \int_{0}^{\infty} e^{-s z}\left\langle e^{i s \mathcal{H}} \phi, \psi\right\rangle d s d z \\
& =\frac{1}{\pi} \int_{0}^{\infty} e^{(t-s) b} \frac{\sin ((t-s) R)}{t-s}\left\langle e^{i s \mathcal{H}} \phi, \psi\right\rangle d s .
\end{aligned}
$$

Since $e^{(t-s) b}\left\langle e^{i s \mathcal{H}} \phi, \psi\right\rangle$ is a $C^{1}$ function in $s$ (recall $\phi \in \operatorname{Dom}(\mathcal{H})$ ) as well as exponentially decaying in $s$ (because of $b>a$ ), it follows from standard properties of the Dirichlet kernel that the limit in (6.13) exists and equals $\left\langle e^{i t \mathcal{H}} \phi, \psi\right\rangle$, as claimed. Note that if $t<0$, then the limit is zero. Therefore, it follows that for any $b>a$,

$$
\begin{gathered}
\left\langle e^{i t \mathcal{H}} \phi, \psi\right\rangle=-\lim _{R \rightarrow \infty}\left\{\frac{1}{2 \pi i} \int_{b-i R}^{b+i R} e^{t z}\left\langle(i \mathcal{H}-z)^{-1} \phi, \psi\right\rangle d z\right. \\
\left.-\frac{1}{2 \pi i} \int_{-b-i R}^{-b+i R} e^{t z}\left\langle(i \mathcal{H}-z)^{-1} \phi, \psi\right\rangle d z\right\} \\
=\lim _{R \rightarrow \infty} \frac{1}{2 \pi i} \int_{-R}^{R} e^{i t \lambda}\left\langle\left[e^{-b t}(\mathcal{H}-(\lambda+i b))^{-1}-e^{b t}(\mathcal{H}-(\lambda-i b))^{-1}\right] \phi, \psi\right\rangle d \lambda .
\end{gathered}
$$

Next, assume that $\phi, \psi$ are as in the statement of the theorem, and shift the contour in the previous integrals by sending $b \rightarrow 0+$. More precisely, we apply the residue theorem to the contour integrals over the rectangles with vertices $\pm R+i b, \pm R+i 0$ and the reflected one below the real axis. The horizontal segments on the real axis need to avoid the poles, which can be achieved by surrounding each of the at most finitely many real poles of the resolvent $(\mathcal{H}-z)^{-1}$ by a small semi-circle. Combining each such semi-circle with its reflection yields a small closed loop and the resulting integral is precisely the Riesz projection corresponding to that real eigenvalue. The Riesz projections corresponding to eigenvalues on the imaginary axis are obtained as residues. On the other hand, we also need to show that the contribution by the horizontal segments is zero in the limit $R \rightarrow \infty$. This, however, follows from the limiting absorption principle (6.9). The lemma follows.

Under our assumptions, $\mathcal{H}$ can only have finitely many points in its discrete spectrum, each of which is an eigenvalue of finite algebraic multiplicity (however, the geometric and algebraic multiplicities my differ for each one of them). Let $P_{d}$ denote the Riesz projection onto the discrete spectrum. It is given by the Cauchy integral of the resolvent around a simple closed curve which surrounds the entire discrete spectrum but avoids the essential spectrum. We write $P_{s}=I-P_{d}$ for the "stable" projection.

We can now state the Fourier expansion theorem. So far, our analysis has been restricted to the right half of the essential spectrum, i.e., $[1, \infty)$. To extend this to 
the left half, it will be convenient (but not essential) to use a further property of $V$; see Definition 5.1 and (5.2). Namely,

$$
\sigma_{1} V \sigma_{1}=-V \text { and } \sigma_{1} \mathcal{H} \sigma_{1}=-\mathcal{H} \text { where } \sigma_{1}=\left(\begin{array}{cc}
0 & 1 \\
1 & 0
\end{array}\right) .
$$

Therefore, if we denote the scattering solutions of Lemma 6.3 by $\mathcal{F}_{+}(x, \lambda), \mathcal{G}_{+}(x, \lambda)$, then the corresponding ones for the negative essential spectrum are

$$
\mathcal{F}_{-}(x, \lambda):=\sigma_{1} \mathcal{F}_{+}(x, \lambda), \quad \mathcal{G}_{-}(x, \lambda):=\sigma_{1} \mathcal{G}_{+}(x, \lambda) .
$$

Proposition 6.9. Let

$$
e_{ \pm}(x, \lambda)= \begin{cases}\mathcal{F}_{ \pm}(x, \lambda) & \text { if } \lambda \geq 0 \\ \mathcal{G}_{ \pm}(x,-\lambda) & \text { if } \lambda \leq 0\end{cases}
$$

Then for every $\phi, \psi \in \mathcal{S}$,

$$
\begin{aligned}
\left\langle P_{s} \phi, \psi\right\rangle & =\frac{1}{2 \pi} \int_{-\infty}^{\infty}\left\langle\phi, \sigma_{3} e_{+}(\cdot, \lambda)\right\rangle \overline{\left\langle\psi, e_{+}(\cdot, \lambda)\right\rangle} d \lambda \\
& +\frac{1}{2 \pi} \int_{-\infty}^{\infty}\left\langle\phi, \sigma_{3} e_{-}(\cdot, \lambda)\right\rangle \overline{\left\langle\psi, e_{-}(\cdot, \lambda)\right\rangle} d \lambda .
\end{aligned}
$$

The integrals on the right-hand side are absolutely convergent. In fact, the integrand is rapidly decaying.

Proof. We start from the representation formula

$$
\begin{aligned}
\left\langle P_{s} \phi, \psi\right\rangle & =\frac{1}{2 \pi i}\left\{\int_{-\infty}^{-1}+\int_{1}^{\infty}\right\}\left\langle\left((\mathcal{H}-(u+i 0))^{-1}-(\mathcal{H}-(u-i 0))^{-1}\right) \phi, \psi\right\rangle d u \\
= & \frac{1}{2 \pi i} \int_{0}^{\infty} 2 \lambda\left\langle\left(\left(\mathcal{H}-\left(\lambda^{2}+1+i 0\right)\right)^{-1}-\left(\mathcal{H}-\left(\lambda^{2}+1-i 0\right)\right)^{-1}\right) \phi, \psi\right\rangle d \lambda \\
& +\frac{1}{2 \pi i} \int_{0}^{\infty} 2 \lambda\left\langle\left(\left(\mathcal{H}-\left(-\lambda^{2}-1+i 0\right)\right)^{-1}-\left(\mathcal{H}-\left(-\lambda^{2}-1-i 0\right)\right)^{-1}\right) \phi, \psi\right\rangle d \lambda
\end{aligned}
$$

which holds in the principal value sense. This was proved in 14. Since for $\lambda>0$

$$
\begin{aligned}
& \left(\mathcal{H}-\left(\lambda^{2}+1+i 0\right)\right)^{-1}(x, y)-\left(\mathcal{H}-\left(\lambda^{2}+1-i 0\right)\right)^{-1}(x, y) \\
& =\frac{-1}{2 i \lambda}\left[e_{+}(x, \lambda) e_{+}(x,-\lambda)\right]\left[e_{+}(y, \lambda) e_{+}(y,-\lambda)\right]^{*} \sigma_{3}, \\
& \left(\mathcal{H}-\left(-\lambda^{2}-1+i 0\right)\right)^{-1}(x, y)-\left(\mathcal{H}-\left(-\lambda^{2}-1-i 0\right)\right)^{-1}(x, y) \\
& =\frac{-1}{2 i \lambda}\left[e_{-}(x, \lambda) e_{-}(x,-\lambda)\right]\left[e_{-}(y, \lambda) e_{-}(y,-\lambda)\right]^{*} \sigma_{3},
\end{aligned}
$$

this representation formula can be rewritten as

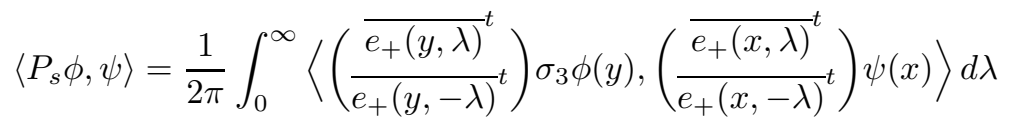

$$
\begin{aligned}
& +\frac{1}{2 \pi} \int_{0}^{\infty}\left\langle\left({\overline{e_{-}(y, \lambda)}}^{t} t\right) \sigma_{3} \phi(y),\left({\frac{\bar{e}_{-}(x, \lambda)}{e_{-}(x,-\lambda)}}^{t}\right) \psi(x)\right\rangle d \lambda \\
& =\frac{1}{2 \pi} \int_{-\infty}^{\infty}\left\langle\phi, \sigma_{3} e_{+}(\cdot, \lambda)\right\rangle \overline{\left\langle\psi, e_{+}(\cdot, \lambda)\right\rangle} d \lambda+\frac{1}{2 \pi} \int_{-\infty}^{\infty}\left\langle\phi, \sigma_{3} e_{-}(\cdot, \lambda)\right\rangle \overline{\left\langle\psi, e_{-}(\cdot, \lambda)\right\rangle} d \lambda,
\end{aligned}
$$


as claimed. So far, we need to interpret the right-hand side in the principal value sense. Since $e_{ \pm}$are solutions, i.e., they satisfy $\mathcal{H} e_{ \pm}(\cdot, \lambda)=\left(1+\lambda^{2}\right) e_{ \pm}(\cdot, \lambda)$, we obtain

$$
\left\langle\phi, \sigma_{3} e_{+}\right\rangle=\left\langle\mathcal{H}^{m} \phi, \sigma_{3} e_{+}\right\rangle\left(1+\lambda^{2}\right)^{m}
$$

and the rapid decay follows.

In fact, the same proof also yields the following representation of the time evolution.

Corollary 6.10. With the same notation as in the previous theorem,

$$
\begin{aligned}
\left\langle e^{i t \mathcal{H}} P_{s} \phi, \psi\right\rangle & =\frac{e^{i t}}{2 \pi} \int_{-\infty}^{\infty} e^{i t \lambda^{2}}\left\langle\phi, \sigma_{3} e_{+}(\cdot, \lambda)\right\rangle \overline{\left\langle\psi, e_{+}(\cdot, \lambda)\right\rangle} d \lambda \\
& +\frac{e^{-i t}}{2 \pi} \int_{-\infty}^{\infty} e^{-i t \lambda^{2}}\left\langle\phi, \sigma_{3} e_{-}(\cdot, \lambda)\right\rangle \overline{\left\langle\psi, e_{-}(\cdot, \lambda)\right\rangle} d \lambda,
\end{aligned}
$$

with absolutely convergent integrals.

Formally, this can be written as

$$
\begin{aligned}
e^{i t \mathcal{H}} P_{s} \phi & =\frac{e^{i t}}{2 \pi} \int_{-\infty}^{\infty} e^{i t \lambda^{2}}\left\langle\phi, \sigma_{3} e_{+}(\cdot, \lambda)\right\rangle e_{+}(\cdot, \lambda) d \lambda \\
& +\frac{e^{-i t}}{2 \pi} \int_{-\infty}^{\infty} e^{-i t \lambda^{2}}\left\langle\phi, \sigma_{3} e_{-}(\cdot, \lambda)\right\rangle e_{-}(\cdot, \lambda) d \lambda
\end{aligned}
$$

One easy consequence of Proposition 6.9 is the following stability bound on the evolution. Not too surprisingly, it can also be established independently of the scattering theory from above. In fact, it is a relatively straightforward consequence of Kato's smoothing theory which does not depend on the dimension. See [14 for the three-dimensional case. The argument which is presented there only uses the representation from Lemma 6.8 and carries over to the one-dimensional case as well.

Lemma 6.11. Let $\mathcal{H}$ be admissible. Then the following stability bound holds:

$$
\sup _{t \in \mathbb{R}}\left\|e^{i t \mathcal{H}} P_{s}\right\|_{2 \rightarrow 2} \leq C .
$$

Proof. In view of Corollary 6.10

$$
\begin{aligned}
& \left|\left\langle e^{i t \mathcal{H}} P_{s} \phi, \psi\right\rangle\right| \\
& \leq \int_{-\infty}^{\infty}\left|\left\langle\phi, \sigma_{3} e_{+}(\cdot, \lambda)\right\rangle\right|\left|\left\langle\psi, e_{+}(\cdot, \lambda)\right\rangle\right| d \lambda+\int_{-\infty}^{\infty}\left|\left\langle\phi, \sigma_{3} e_{-}(\cdot, \lambda)\right\rangle\right|\left|\left\langle\psi, e_{-}(\cdot, \lambda)\right\rangle\right| d \lambda \\
& \leq 2 \max _{ \pm}\left(\int_{-\infty}^{\infty}\left|\left\langle\phi, \sigma_{3} e_{ \pm}(\cdot, \lambda)\right\rangle\right|^{2} d \lambda\right)^{\frac{1}{2}}\left(\int_{-\infty}^{\infty}\left|\left\langle\psi, e_{ \pm}(\cdot, \lambda)\right\rangle\right| d \lambda\right)^{\frac{1}{2}} \leq C\|\phi\|_{2}\|\psi\|_{2}
\end{aligned}
$$

where the final bound follows from the asymptotics of Lemma 6.3.

Next, we state the natural bound on $x e^{i t \mathcal{H}} P_{s}$ :

Lemma 6.12. Let $\mathcal{H}$ be admissible. Then

$$
\left\|x e^{i t \mathcal{H}} P_{s} f\right\|_{2} \leq C\langle t\rangle\|f\|_{H^{1}}+C\|x f\|_{2}
$$

for all $t \geq 0$. 
Proof. Clearly,

$$
\begin{aligned}
& i \frac{d}{d t}\left\langle|x|^{2} e^{i t \mathcal{H}} P_{s} f, e^{i t \mathcal{H}} P_{s} f\right\rangle \\
& =\left\langle\left(\mathcal{H}^{*}|x|^{2}-|x|^{2} \mathcal{H}\right) e^{i t \mathcal{H}} P_{s} f, e^{i t \mathcal{H}} P_{s} f\right\rangle \\
& =-\left\langle\left[\partial_{x}^{2},|x|^{2}\right] \sigma_{3} e^{i t \mathcal{H}} P_{s} f, e^{i t \mathcal{H}} P_{s} f\right\rangle+\left\langle\left(V^{*}-V\right)|x|^{2} e^{i t \mathcal{H}} P_{s} f, e^{i t \mathcal{H}} P_{s} f\right\rangle \\
& =-\left\langle\left(2+2 x \partial_{x}\right) \sigma_{3} e^{i t \mathcal{H}} P_{s} f, e^{i t \mathcal{H}} P_{s} f\right\rangle+\left\langle\left(V^{*}-V\right)|x|^{2} e^{i t \mathcal{H}} P_{s} f, e^{i t \mathcal{H}} P_{s} f\right\rangle .
\end{aligned}
$$

In particular,

$$
\begin{aligned}
& \left|i \frac{d}{d t}\left\langle|x|^{2} e^{i t \mathcal{H}} P_{s} f, e^{i t \mathcal{H}} P_{s} f\right\rangle\right|_{t=0} \mid \\
& \leq\left|\left\langle\left(2+2 x \partial_{x}\right) \sigma_{3} P_{s} f, P_{s} f\right\rangle\right|+\left|\left\langle\left(V^{*}-V\right)|x|^{2} P_{s} f, P_{s} f\right\rangle\right| \\
& \leq C\left(\|\langle x\rangle f\|_{2}\|f\|_{H^{1}}+\|f\|_{2}^{2}\right) .
\end{aligned}
$$

Thus,

$$
\begin{aligned}
- & \frac{d^{2}}{d t^{2}}\left\langle|x|^{2} e^{i t \mathcal{H}} P_{s} f, e^{i t \mathcal{H}} P_{s} f\right\rangle \\
= & -2\left\langle\left(\mathcal{H}^{*} x \partial_{x}-x \partial_{x} \mathcal{H}^{*}\right) \sigma_{3} e^{i t \mathcal{H}} P_{s} f, e^{i t \mathcal{H}} P_{s} f\right\rangle \\
& +\left\langle\left(\mathcal{H}^{*}\left(V^{*}-V\right)|x|^{2}-\left(V^{*}-V\right)|x|^{2} \mathcal{H}\right) e^{i t \mathcal{H}} P_{s} f, e^{i t \mathcal{H}} P_{s} f\right\rangle \\
= & 2\left\langle\left(\partial_{x}^{2}\left(x \partial_{x}\right)-x \partial_{x} \partial_{x}^{2}\right) e^{i t \mathcal{H}} P_{s} f, e^{i t \mathcal{H}} P_{s} f\right\rangle+2\left\langle x \partial_{x}\left(V^{*}\right) \sigma_{3} e^{i t \mathcal{H}} P_{s} f, e^{i t \mathcal{H}} P_{s} f\right\rangle \\
& -\left\langle\left(\partial_{x}^{2} \sigma_{3}\left(V^{*}-V\right)|x|^{2}-\left(V^{*}-V\right)|x|^{2} \sigma_{3} \partial_{x}^{2}\right) e^{i t \mathcal{H}} P_{s} f, e^{i t \mathcal{H}} P_{s} f\right\rangle \\
+ & \left\langle\left(V^{*}\left(V^{*}-V\right)|x|^{2}-\left(V^{*}-V\right)|x|^{2} V\right) e^{i t \mathcal{H}} P_{s} f, e^{i t \mathcal{H}} P_{s} f\right\rangle,
\end{aligned}
$$

which implies that

$$
\sup _{t}\left|\frac{d^{2}}{d t^{2}}\left\langle|x|^{2} e^{i t \mathcal{H}} P_{s} f, e^{i t \mathcal{H}} P_{s} f\right\rangle\right| \leq \sup _{t} C\left\|e^{i t \mathcal{H}} P_{s} f\right\|_{H^{1}}^{2} \leq C\|f\|_{H^{1}}^{2}
$$

A Taylor expansion of degree two therefore yields

$$
\begin{aligned}
\left\|x e^{i t \mathcal{H}} P_{s} f\right\|_{2}^{2} & \leq C\|\langle x\rangle f\|_{2}^{2}+C t\left(\|\langle x\rangle f\|_{2}\|f\|_{H^{1}}+\|f\|_{2}^{2}\right)+C t^{2}\|f\|_{H^{1}}^{2} \\
& \leq C\left(\langle t\rangle^{2}\|f\|_{H^{1}}^{2}+\|\langle x\rangle f\|_{2}^{2}\right)
\end{aligned}
$$

and the lemma follows.

\section{Dispersive estimates: The unweighted Case}

In this section, we prove dispersive estimates on $e^{i t \mathcal{H}} P_{s}$ in the $L^{1}(\mathbb{R}) \rightarrow L^{\infty}(\mathbb{R})$ sense. For the scalar case, see for example Weder [54, Artbazar and Yajima [1, and Goldberg and Schlag [24].

Proposition 7.1. Let $\mathcal{H}$ be admissible; see Definition 6.1. Then for all $t \neq 0$,

$$
\left\|e^{i t \mathcal{H}} P_{s}\right\|_{1 \rightarrow \infty} \leq C|t|^{-\frac{1}{2}}
$$

with some $C=C(V)$. 
Proof. We will follow the proof strategy of the one-dimensional case of [24]. To do so, we start from the representation formula

$$
\begin{aligned}
& \left\langle e^{i t \mathcal{H}} P_{s} \phi, \psi\right\rangle \\
& =\frac{e^{i t}}{\pi i} \int_{0}^{\infty} e^{i t \lambda^{2}} \lambda\left\langle\left(\left(\mathcal{H}-\left(\lambda^{2}+1+i 0\right)\right)^{-1}-\left(\mathcal{H}-\left(\lambda^{2}+1-i 0\right)\right)^{-1}\right) \phi, \psi\right\rangle d \lambda \\
& +\frac{e^{-i t}}{\pi i} \int_{0}^{\infty} e^{-i t \lambda^{2}} \lambda\left\langle\left(\left(\mathcal{H}-\left(-\lambda^{2}-1+i 0\right)\right)^{-1}-\left(\mathcal{H}-\left(-\lambda^{2}-1-i 0\right)\right)^{-1}\right) \phi, \psi\right\rangle d \lambda,
\end{aligned}
$$

which holds in the principal value sense if $\phi, \psi \in \mathcal{S}$. It will suffice to deal with energies $E \geq 1$ since the second integral will satisfy the same bounds as the first by symmetry. Let $\chi$ be a smooth, even, and compactly supported bump function so that $\chi(\lambda)=1$ for $|\lambda| \leq \lambda_{1}$, where $\lambda_{1}=\lambda_{1}(V)$ will be specified later. On the support of $1-\chi(\lambda)$ (the "high energy case") we will use a Born series expansion. More precisely, since

$$
\begin{aligned}
& \left(\mathcal{H}_{0}-\left(\lambda^{2}+1 \pm i 0\right)\right)^{-1}(x, y) \\
& =\left(\begin{array}{cc}
\left(-\partial_{x x}-\left(\lambda^{2} \pm i 0\right)\right)^{-1}(x, y) & 0 \\
0 & \left(-\partial_{x x}-\mu^{2}\right)^{-1}(x, y)
\end{array}\right) \\
& =\left(\begin{array}{cc}
\mp \frac{e^{ \pm i \lambda|x-y|}}{2 i \lambda} & 0 \\
0 & -\frac{e^{-\mu|x-y|}}{2 \mu}
\end{array}\right)
\end{aligned}
$$

satisfies

$$
\left\|\left(\mathcal{H}_{0}-\left(\lambda^{2}+1 \pm i 0\right)\right)^{-1}\right\|_{1 \rightarrow \infty} \leq C|\lambda|^{-1},
$$

we conclude that

$$
\left(\mathcal{H}-\left(\lambda^{2}+1 \pm i 0\right)\right)^{-1}=\sum_{n=0}^{\infty}(-1)^{n}\left(\mathcal{H}_{0}-\left(\lambda^{2}+1+i 0\right)\right)^{-1}\left(V\left(\mathcal{H}_{0}-\left(\lambda^{2}+1+i 0\right)\right)^{-1}\right)^{n}
$$

converges in the operator norm of $L^{1}(\mathbb{R}) \rightarrow L^{\infty}(\mathbb{R})$ provided $|\lambda| \geq \lambda_{1}$ is sufficiently large compared to $\|V\|_{L^{1}}$. Indeed, the operator norms of the $n$-th summand on the right-hand side is bounded by $C \lambda^{-1}\left(\|V\|_{1} \lambda^{-1}\right)^{n}$. Hence 22

$$
\begin{aligned}
& \left|\left\langle e^{i t \mathcal{H}}(1-\chi(H-I)) P_{s}^{+} \phi, \psi\right\rangle\right| \\
& \leq C \sum_{n=0}^{\infty} \mid \int_{0}^{\infty} e^{i t \lambda^{2}} \lambda\left(1-\chi\left(\lambda^{2}\right)\right)\left[\left\langle\left(\mathcal{H}_{0}-\left(\lambda^{2}+1+i 0\right)\right)^{-1}\left(V\left(\mathcal{H}_{0}-\left(\lambda^{2}+1+i 0\right)\right)^{-1}\right)^{n} \phi, \psi\right\rangle\right. \\
& \left.\quad-\left\langle\left(\mathcal{H}_{0}-\left(\lambda^{2}+1-i 0\right)\right)^{-1}\left(V\left(\mathcal{H}_{0}-\left(\lambda^{2}+1-i 0\right)\right)^{-1}\right)^{n} \phi, \psi\right\rangle\right] d \lambda \mid .
\end{aligned}
$$

The term $n=0$ represents the usual free Schrödinger decay and its contribution is bounded by $C|t|^{-\frac{1}{2}}\|\phi\|_{1}\|\psi\|_{1}$. Indeed, the oscillatory integral bound that arises in this case is

$$
\begin{aligned}
& \sup _{x, y \in \mathbb{R}}\left|\int_{0}^{\infty} e^{i t \lambda^{2}}\left(1-\chi\left(\lambda^{2}\right)\right) \cos (\lambda|x-y|) d \lambda\right| \\
& \leq \sup _{x, y \in \mathbb{R}}\left|\int_{-\infty}^{\infty}\left[e^{i t \lambda^{2}}\right]^{\vee}(u)\left[\left(1-\chi\left(\lambda^{2}\right)\right) \cos (\lambda|x-y|)\right]^{\vee}(u) d u\right| \leq C|t|^{-\frac{1}{2}} .
\end{aligned}
$$

\footnotetext{
${ }^{22}$ Symbols like $1-\chi(H-I)$ and $P_{s}^{+}$are being used in a purely formal way - they are defined by the $\lambda$-integrals in which they arise.
} 
Here the Fourier transforms are with respect to $\lambda$ and we used that

$$
\left[\left(1-\chi\left(\lambda^{2}\right)\right) \cos (\lambda a)\right]^{\vee}(u)
$$

is a measure with total variation norm uniformly bounded in $a$. Next, consider the contribution by $n=1$ in (7.2). Writing $\phi=\left(\begin{array}{l}\phi_{1} \\ \phi_{2}\end{array}\right), \psi=\left(\begin{array}{l}\psi_{1} \\ \psi_{2}\end{array}\right)$, this term becomes (we ignore multiplicative constants and we write $d x=d x_{0} d x_{1} d x_{2}$ for simplicity)

$$
\int_{\mathbb{R}^{2}} \int_{-\infty}^{\infty} e^{i t \lambda^{2}} \lambda^{-1}\left(1-\chi\left(\lambda^{2}\right)\right) \sin \left(\lambda\left(\left|x_{0}-x_{1}\right|+\left|x_{1}-x_{2}\right|\right)\right) d \lambda V_{1}\left(x_{1}\right) \phi_{1}\left(x_{0}\right) \bar{\psi}_{1}\left(x_{2}\right) d x
$$

$+\int_{\mathbb{R}^{2}} \int_{-\infty}^{\infty} e^{i t \lambda^{2}} \lambda^{-1}\left(1-\chi\left(\lambda^{2}\right)\right) \sin \left(\lambda\left|x_{0}-x_{1}\right|\right) e^{-\mu\left|x_{2}-x_{1}\right|} d \lambda V_{2}\left(x_{1}\right) \phi_{1}\left(x_{0}\right) \bar{\psi}_{2}\left(x_{2}\right) d x$

$$
-\int_{\mathbb{R}^{2}} \int_{-\infty}^{\infty} e^{i t \lambda^{2}} \lambda^{-1}\left(1-\chi\left(\lambda^{2}\right)\right) \sin \left(\lambda\left|x_{2}-x_{1}\right|\right) e^{-\mu\left|x_{1}-x_{0}\right|} d \lambda V_{2}\left(x_{1}\right) \phi_{2}\left(x_{0}\right) \bar{\psi}_{1}\left(x_{2}\right) d x
$$

To bound the oscillatory integrals, note first that

$$
\begin{aligned}
& \left\|\left(1+u^{2}\right)\left[\lambda^{-1}\left(1-\chi\left(\lambda^{2}\right)\right)\right]^{\vee}(u)\right\|_{\infty} \\
& \leq\left\|\left[\lambda^{-1}\left(1-\chi\left(\lambda^{2}\right)\right)\right]^{\vee}(u)\right\|_{\infty}+C\left\|\partial_{\lambda}^{2}\left[\lambda^{-1}\left(1-\chi\left(\lambda^{2}\right)\right)\right]\right\|_{1}<\infty,
\end{aligned}
$$

since $\left[\lambda^{-1}\right]^{\vee}(u)=c \operatorname{sign}(u)$. Hence

$$
\left\|\left[\lambda^{-1}\left(1-\chi\left(\lambda^{2}\right)\right)\right]^{\vee}(u)\right\|_{1}<\infty .
$$

Second, we claim that (recall $\mu=\sqrt{2+\lambda^{2}}$ )

$$
\sup _{a \geq 0}\left\|\int_{-\infty}^{\infty} e^{-a \sqrt{2+\lambda^{2}}} e^{-i \lambda u} d \lambda\right\|_{\mathcal{M}}=\sup _{b \geq 0}\left\|\int_{-\infty}^{\infty} e^{-\sqrt{b+\lambda^{2}}} e^{-i \lambda u} d \lambda\right\|_{\mathcal{M}}<\infty
$$

where the norms refer to the total variation norms of measures. To see this, compute

$$
\begin{aligned}
\left\|\partial_{\lambda}^{2} e^{-\sqrt{b+\lambda^{2}}}\right\|_{1} & =\int_{-\infty}^{\infty}\left|\left(-\frac{b}{\left(b+\lambda^{2}\right)^{\frac{3}{2}}}+\frac{\lambda^{2}}{b+\lambda^{2}}\right) e^{-\sqrt{b+\lambda^{2}}}\right| d \lambda \\
& \leq \int_{-\infty}^{\infty} \frac{b}{\left(b+\lambda^{2}\right)^{\frac{3}{2}}} d \lambda+\int_{-\infty}^{\infty} e^{-\sqrt{b+\lambda^{2}}} d \lambda \\
& \leq \int_{-\infty}^{\infty} \frac{1}{\left(1+\lambda^{2}\right)^{\frac{3}{2}}} d \lambda+\int_{-\infty}^{\infty} e^{-|\lambda|} d \lambda \leq C
\end{aligned}
$$

uniformly in $b>0$. It follows that

$$
\sup _{b \geq 0}\left(1+u^{2}\right)\left|\int_{-\infty}^{\infty} e^{-\sqrt{b+\lambda^{2}}} e^{-i \lambda u} d \lambda\right| \leq C
$$

and (7.6) holds. By the same type of argument as in the $n=0$ case, we conclude that the contribution from (7.3) $-(7.5)$ is

$$
\leq C|t|^{-\frac{1}{2}}\|V\|_{1}\|\phi\|_{1}\|\psi\|_{1}
$$

as desired. Finally, the terms $n \geq 2$ in (7.2) are similar. More precisely, they lead to oscillatory integrals of the form

$$
\sup _{b \geq 0, a \in \mathbb{R}}\left|\int_{-\infty}^{\infty} e^{i t \lambda^{2}} \lambda^{-n}\left(1-\chi\left(\lambda^{2}\right)\right) e^{i a \lambda} e^{-b \sqrt{2+\lambda^{2}}} d \lambda\right| \leq C \lambda_{1}^{-n}|t|^{-\frac{1}{2}},
$$


which follow by the same type of arguments as before. Hence, the entire series in (7.2) is estimated by

$$
\sum_{n=0}^{\infty} C \lambda_{1}^{-n}|t|^{-\frac{1}{2}}\|V\|_{1}^{n}\|\phi\|_{1}\|\psi\|_{1} \leq C|t|^{-\frac{1}{2}}\|\phi\|_{1}\|\psi\|_{1}
$$

as claimed.

It remains to deal with those $\lambda$ that belong to the support of $\chi$. By Lemma 6.5,

$$
\begin{aligned}
& \left\langle e^{i t \mathcal{H}} \chi(\mathcal{H}-I) P_{s}^{+} \phi, \psi\right\rangle \\
& =\frac{e^{i t}}{\pi i} \int_{0}^{\infty} e^{i t \lambda^{2}} \lambda \chi\left(\lambda^{2}\right)\left\langle\left[\left(\mathcal{H}-\left(\lambda^{2}+1+i 0\right)\right)^{-1}-\left(\mathcal{H}-\left(\lambda^{2}+1-i 0\right)\right)^{-1}\right] \phi, \psi\right\rangle d \lambda
\end{aligned}
$$

$$
=-\frac{e^{i t}}{\pi i} \int_{\mathbb{R}^{2}} \int_{-\infty}^{\infty} e^{i t \lambda^{2}} \lambda \chi\left(\lambda^{2}\right)\left\langle F_{1}(x, \lambda) D^{-1}(\lambda) G_{2}^{t}(y, \lambda) \sigma_{3} \phi(x), \psi(y)\right\rangle d \lambda \chi_{[x \geq y]} d x d y
$$

$$
+\frac{e^{i t}}{\pi i} \int_{\mathbb{R}^{2}} \int_{-\infty}^{\infty} e^{i t \lambda^{2}} \lambda \chi\left(\lambda^{2}\right)\left\langle G_{2}(x, \lambda) D^{-1}(\lambda) F_{1}^{t}(y, \lambda) \sigma_{3} \phi(x), \psi(y)\right\rangle d \lambda \chi_{[x<y]} d x d y .
$$

The second integral (7.8) can be transformed into a variant of the first (7.7) by means of the change of variables $x \rightarrow-x, y \rightarrow-y$ :

$$
\begin{aligned}
& \int_{\mathbb{R}^{2}} \int_{-\infty}^{\infty} e^{i t \lambda^{2}} \lambda \chi\left(\lambda^{2}\right)\left\langle G_{2}(x, \lambda) D^{-1}(\lambda) F_{1}^{t}(y, \lambda) \sigma_{3} \phi(x), \psi(y)\right\rangle d \lambda \chi_{[x<y]} d x d y \\
& =\int_{\mathbb{R}^{2}} \int_{-\infty}^{\infty} e^{i t \lambda^{2}} \lambda \chi\left(\lambda^{2}\right)\left\langle F_{1}(x, \lambda) D^{-1}(\lambda) G_{2}^{t}(y, \lambda) \sigma_{3} \phi(-x), \psi(-y)\right\rangle d \lambda \chi_{[x>y]} d x d y .
\end{aligned}
$$

Hence it suffices to bound the first. To this end we need to consider three cases: $x \geq 0 \geq y, 0 \geq x \geq y$, and $x \geq y \geq 0$.

Case 1. $x \geq 0 \geq y$.

We write the $\lambda$-integral in (7.7) as the sum of four pieces, according to the various possibilities for the asymptotic behavior as $x \rightarrow \infty$ or $-y \rightarrow \infty$ :

$$
\begin{aligned}
& \int_{-\infty}^{\infty} e^{i t \lambda^{2}} \lambda \chi\left(\lambda^{2}\right) F_{1}(x, \lambda) D^{-1}(\lambda) G_{2}^{t}(y, \lambda) \sigma_{3} d \lambda \\
& =\int_{-\infty}^{\infty} e^{i t \lambda^{2}} \lambda \chi\left(\lambda^{2}\right)\left[f_{1}(x, \lambda) 0\right] D^{-1}(\lambda)\left[f_{1}(-y, \lambda) 0\right]^{t} \sigma_{3} d \lambda \\
& \quad+\int_{-\infty}^{\infty} e^{i t \lambda^{2}} \lambda \chi\left(\lambda^{2}\right)\left[f_{1}(x, \lambda) 0\right] D^{-1}(\lambda)\left[0 f_{3}(-y, \lambda)\right]^{t} \sigma_{3} d \lambda \\
& \quad+\int_{-\infty}^{\infty} e^{i t \lambda^{2}} \lambda \chi\left(\lambda^{2}\right)\left[0 f_{3}(x, \lambda)\right] D^{-1}(\lambda)\left[f_{1}(-y, \lambda) 0\right]^{t} \sigma_{3} d \lambda \\
& \quad+\int_{-\infty}^{\infty} e^{i t \lambda^{2}} \lambda \chi\left(\lambda^{2}\right)\left[0 f_{3}(x, \lambda)\right] D^{-1}(\lambda)\left[0 f_{3}(-y, \lambda)\right]^{t} \sigma_{3} d \lambda .
\end{aligned}
$$


Now pick another cut-off function $\tilde{\chi}$ so that $\tilde{\chi} \chi=\chi$. Then (7.9) is estimated as follows (with the Fourier transform relative to $\lambda$ ):

$$
\begin{aligned}
& |\underline{(7.9)}| \leq\left|\int_{-\infty}^{\infty} e^{i\left[t \lambda^{2}+\lambda(x-y)\right]} \lambda \chi\left(\lambda^{2}\right)\left[e^{-i \lambda x} f_{1}(x, \lambda) 0\right] D^{-1}(\lambda)\left[e^{i \lambda y} f_{1}(-y, \lambda) 0\right]^{t} \sigma_{3} d \lambda\right| \\
& \begin{aligned}
& \leq C|t|^{-\frac{1}{2}} \int_{-\infty}^{\infty} \mid\left[\lambda \chi\left(\lambda^{2}\right) D^{-1}(\lambda)\right]^{\vee} *\left[\tilde{\chi}\left(\lambda^{2}\right) e^{-i \lambda x} f_{1}(x, \lambda)\right]^{\vee} \\
&(7.13) *\left[\tilde{\chi}\left(\lambda^{2}\right) e^{i \lambda y} f_{1}(-y, \lambda)\right]^{\vee}(\tilde{\xi}) \mid d \tilde{\xi} \\
& \leq C|t|^{-\frac{1}{2}}\left\|\left[\lambda \chi\left(\lambda^{2}\right) D^{-1}(\lambda)\right]^{\vee}\right\|_{1}\left\|\left[\tilde{\chi}\left(\lambda^{2}\right) e^{-i \lambda x} f_{1}(x, \lambda)\right]^{\vee}\right\|_{1}\left\|\left[\tilde{\chi}\left(\lambda^{2}\right) e^{i \lambda y} f_{1}(-y, \lambda)\right]^{\vee}\right\|_{1} .
\end{aligned}
\end{aligned}
$$

Since $D^{-1}(\lambda)$ is smooth, the first norm on the right-hand side of (7.13) is simply a constant. On the other hand, we claim that

$$
\sup _{x \geq 0}\left\|\left[\tilde{\chi}\left(\lambda^{2}\right) e^{-i \lambda x} f_{1}(x, \lambda)\right]^{\vee}\right\|_{1} \leq C \text { and } \sup _{y \leq 0}\left\|\left[\tilde{\chi}\left(\lambda^{2}\right) e^{i \lambda y} f_{1}(-y, \lambda)\right]^{\vee}\right\|_{1} \leq C .
$$

This is an easy consequence of Lemma 5.3. Indeed, (5.9) insures that

$$
\left|\partial_{\lambda}^{2}\left[\tilde{\chi}\left(\lambda^{2}\right) e^{-i \lambda x} f_{1}(x, \lambda)\right]\right| \leq C x^{2} e^{-\gamma x}
$$

for all $\lambda \in \mathbb{R}$ and all $x \geq x_{0}$. Since we are dealing with a compact $\lambda$-interval, it also follows that

$$
\sup _{x \geq 0, \lambda \in \mathbb{R}}\left|\left(1-\partial_{\lambda}^{2}\right)\left[\tilde{\chi}\left(\lambda^{2}\right) e^{-i \lambda x} f_{1}(x, \lambda)\right]\right| \leq C .
$$

Hence,

and thus

$$
\sup _{\tilde{\xi} \in \mathbb{R}, x \geq 0}\left(1+\tilde{\xi}^{2}\right)\left|\left[\tilde{\chi}\left(\lambda^{2}\right) e^{-i \lambda x} f_{1}(x, \lambda)\right]^{\vee}(\tilde{\xi})\right| \leq C
$$

$$
\sup _{x \geq 0}\left\|\left[\tilde{\chi}\left(\lambda^{2}\right) e^{-i \lambda x} f_{1}(x, \lambda)\right]^{\vee}\right\|_{1} \leq C,
$$

as claimed. Hence the right-hand side of $(7.13)$ is at most $C|t|^{-\frac{1}{2}}$, as desired.

For the second term (7.10) we estimate (recall $y \leq 0$ )

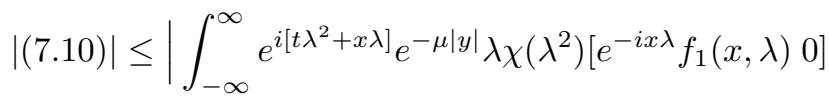

$$
\begin{aligned}
& \times D^{-1}(\lambda)\left[0 e^{\mu|y|} f_{3}(|y|, \lambda)\right]^{t} \sigma_{3} d \lambda \mid \\
& \leq C|t|^{-\frac{1}{2}}\left\|\left[\lambda \chi\left(\lambda^{2}\right) D^{-1}(\lambda) e^{-\mu|y|}\right]^{\vee}\right\|_{1} \\
& \times\left\|\left[\tilde{\chi}\left(\lambda^{2}\right) e^{-i \lambda x} f_{1}(x, \lambda)\right]^{\vee}\right\|_{1}\left\|\left[\tilde{\chi}\left(\lambda^{2}\right) e^{\mu|y|} f_{3}(|y|, \lambda)\right]^{\vee}\right\|_{1} .
\end{aligned}
$$

It follows from (5.3) that

$$
\sup _{y}\left\|\left[\tilde{\chi}\left(\lambda^{2}\right) e^{\mu|y|} f_{3}(|y|, \lambda)\right]^{\vee}\right\|_{1}<\infty .
$$

Furthermore, (7.6) and Lemma 6.2 imply that

$$
\sup _{y}\left\|\left[\lambda \chi\left(\lambda^{2}\right) D^{-1}(\lambda) e^{-\mu|y|}\right]^{\vee}\right\|_{1} \leq C .
$$

Combining these estimates with (7.14) shows that the entire right-hand side of (7.15) is uniformly bounded in $x, y \in \mathbb{R}$. 
The same type of arguments show that

$$
|(\underline{7.11})|+|(17.12)| \leq C|t|^{-\frac{1}{2}}
$$

uniformly in $x \geq 0 \geq y$, which finishes Case 1 .

Case 2. $0 \geq x \geq y$.

In this case, we need to use (5.24). Thus, we write the $\lambda$-integral in (7.7) as the sum of two integrals:

$$
\begin{aligned}
& \int_{-\infty}^{\infty} e^{i t \lambda^{2}} \lambda \chi\left(\lambda^{2}\right) F_{1}(x, \lambda) D^{-1}(\lambda) G_{2}^{t}(y, \lambda) \sigma_{3} d \lambda \\
& =\int_{-\infty}^{\infty} e^{i t \lambda^{2}} \lambda \chi\left(\lambda^{2}\right) G_{1}(x, \lambda) A(\lambda) D^{-1}(\lambda) G_{2}^{t}(y, \lambda) \sigma_{3} d \lambda \\
& \quad+\int_{-\infty}^{\infty} e^{i t \lambda^{2}} \lambda \chi\left(\lambda^{2}\right) G_{2}(x, \lambda) B(\lambda) D^{-1}(\lambda) G_{2}^{t}(y, \lambda) \sigma_{3} d \lambda
\end{aligned}
$$

each of which is itself broken up into four pieces just as in (7.9)-(7.10). Thus, starting with (7.16),

$$
\begin{aligned}
& \int_{-\infty}^{\infty} e^{i t \lambda^{2}} \lambda \chi\left(\lambda^{2}\right) G_{1}(x, \lambda) A(\lambda) D^{-1}(\lambda) G_{2}^{t}(y, \lambda) \sigma_{3} d \lambda \\
& =\int_{-\infty}^{\infty} e^{i t \lambda^{2}} \lambda \chi\left(\lambda^{2}\right)\left[f_{2}(-x, \lambda) 0\right] A(\lambda) D^{-1}(\lambda)\left[f_{1}(-y, \lambda) 0\right]^{t} \sigma_{3} d \lambda \\
& \quad+\int_{-\infty}^{\infty} e^{i t \lambda^{2}} \lambda \chi\left(\lambda^{2}\right)\left[f_{2}(-x, \lambda) 0\right] A(\lambda) D^{-1}(\lambda)\left[0 f_{3}(-y, \lambda)\right]^{t} \sigma_{3} d \lambda \\
& \quad+\int_{-\infty}^{\infty} e^{i t \lambda^{2}} \lambda \chi\left(\lambda^{2}\right)\left[0 f_{4}(-x, \lambda)\right] A(\lambda) D^{-1}(\lambda)\left[f_{1}(-y, \lambda) 0\right]^{t} \sigma_{3} d \lambda \\
& \quad+\int_{-\infty}^{\infty} e^{i t \lambda^{2}} \lambda \chi\left(\lambda^{2}\right)\left[0 f_{4}(-x, \lambda)\right] A(\lambda) D^{-1}(\lambda)\left[0 f_{3}(-y, \lambda)\right]^{t} \sigma_{3} d \lambda
\end{aligned}
$$

We remark that in view of Corollary 5.21 ,

$$
\lambda A(\lambda) D(\lambda)^{-1}=\left(\begin{array}{cc}
\frac{1}{2 i} & 0 \\
0 & \frac{-\lambda}{2 \mu}
\end{array}\right) .
$$

In particular, the expression on the left-hand side is smooth for all $\lambda \in \mathbb{R}$. Moreover, the diagonal form of the right-hand side implies that $(7.19)=(7.20)=0$ (which in the case of (7.20) is crucial), as well as

$$
\begin{aligned}
& (7.18)=\frac{1}{2 i} \int_{-\infty}^{\infty} e^{i\left[t \lambda^{2}+(x-y) \lambda\right]} \chi\left(\lambda^{2}\right)\left[e^{-i x \lambda} f_{2}(-x, \lambda) 0\right]\left[e^{i y \lambda} f_{1}(-y, \lambda) 0\right]^{t} \sigma_{3} d \lambda, \\
& (7.21)=-\int_{-\infty}^{\infty} e^{i t \lambda^{2}} e^{-|x-y| \mu} \frac{\lambda}{2 \mu} \chi\left(\lambda^{2}\right)\left[0 e^{-\mu|x|} f_{4}(|x|, \lambda)\right]\left[0 e^{\mu|y|} f_{3}(|y|, \lambda)\right]^{t} \sigma_{3} d \lambda .
\end{aligned}
$$

The same type of arguments as in Case 1 involving the Fourier transform in $\lambda$ show that both of these expressions are bounded by $C|t|^{-\frac{1}{2}}$ uniformly in $0 \geq x \geq y$. The only new ingredient in this case is the estimate

$$
\sup _{x}\left\|\left[\tilde{\chi}\left(\lambda^{2}\right) e^{-\mu|x|} f_{4}(|x|, \lambda)\right]^{\vee}\right\|_{1}<\infty
$$


which follows from Corollary [5.9. Continuing with (7.17), we write

$$
\begin{aligned}
& \int_{-\infty}^{\infty} e^{i t \lambda^{2}} \lambda \chi\left(\lambda^{2}\right) G_{2}(x, \lambda) B(\lambda) D^{-1}(\lambda) G_{2}^{t}(y, \lambda) \sigma_{3} d \lambda \\
& =\int_{-\infty}^{\infty} e^{i\left[t \lambda^{2}-(x+y) \lambda\right]} \lambda \chi\left(\lambda^{2}\right)\left[e^{i x \lambda} f_{1}(-x, \lambda) 0\right] B(\lambda) D^{-1}(\lambda)\left[e^{i y \lambda} f_{1}(-y, \lambda) 0\right]^{t} \sigma_{3} d \lambda \\
& \quad+\int_{-\infty}^{\infty} e^{i\left[t \lambda^{2}-x \lambda\right]} e^{-\mu|y|} \lambda \chi\left(\lambda^{2}\right)\left[e^{i x \lambda} f_{1}(-x, \lambda) 0\right] B(\lambda) D^{-1}(\lambda)\left[0 e^{\mu|y|} f_{3}(|y|, \lambda)\right]^{t} \sigma_{3} d \lambda \\
& \quad+\int_{-\infty}^{\infty} e^{i\left[t \lambda^{2}-y \lambda\right]} e^{-\mu|x|} \lambda \chi\left(\lambda^{2}\right)\left[0 e^{\mu|x|} f_{3}(|x|, \lambda)\right] B(\lambda) D^{-1}(\lambda)\left[e^{i y \lambda} f_{1}(-y, \lambda) 0\right]^{t} \sigma_{3} d \lambda \\
& \quad+\int_{-\infty}^{\infty} e^{i t \lambda^{2}} e^{-\mu(|x|+|y|)} \lambda \chi\left(\lambda^{2}\right)\left[0 e^{\mu|x|} f_{3}(|x|, \lambda)\right] B(\lambda) D^{-1}(\lambda)\left[0 e^{\mu|y|} f_{3}(|y|, \lambda)\right]^{t} \sigma_{3} d \lambda .
\end{aligned}
$$

We remark that in view of (5.27)

$$
\lambda B(\lambda) D(\lambda)^{-1}=\left(\begin{array}{cc}
-\frac{1}{2 i} & 0 \\
0 & \frac{\lambda}{2 \mu}
\end{array}\right) \mathcal{W}\left[F_{1}(\cdot, \lambda), G_{1}(\cdot, \lambda)\right]^{t} D(\lambda)^{-1}
$$

is a smooth function in $\lambda$. Hence, the same methods as before prove that each of these integrals are bounded by $C|t|^{-\frac{1}{2}}$ uniformly in $0 \geq x \geq y$.

Case 3. $x \geq y \geq 0$.

This case can be reduced to the previous one. Indeed, using that $F_{1}(x, \lambda)=$ $G_{2}(-x, \lambda)$, as well as (5.35), one obtains

$$
\begin{aligned}
& \int_{-\infty}^{\infty} e^{i t \lambda^{2}} \lambda \chi\left(\lambda^{2}\right) F_{1}(x, \lambda) D^{-1}(\lambda) G_{2}^{t}(y, \lambda) \sigma_{3} d \lambda \\
& =\int_{-\infty}^{\infty} e^{i t \lambda^{2}} \lambda \chi\left(\lambda^{2}\right) G_{2}(-x, \lambda) D^{-1}(\lambda) F_{1}^{t}(-y, \lambda) \sigma_{3} d \lambda \\
& =\sigma_{3}\left[\int_{-\infty}^{\infty} e^{i t \lambda^{2}} \lambda \chi\left(\lambda^{2}\right) F_{1}(-y, \lambda) D^{-1}(\lambda) G_{2}^{t}(-x, \lambda) \sigma_{3} d \lambda\right]^{t} \sigma_{3} .
\end{aligned}
$$

Since $0 \geq-y \geq-x$ and since $\sigma_{3}$ and the transpose do not affect the pointwise estimate of Case 2, we are done.

The previous estimate also allows for the introduction of derivatives. We will consider only at most two derivatives, although more are possible.

Corollary 7.2. Let $\mathcal{H}$ be admissible. Then

$$
\left\|e^{i t \mathcal{H}} P_{s} f\right\|_{W^{k, p^{\prime}}(\mathbb{R})} \leq C t^{-\frac{1}{2}\left(\frac{1}{p}-\frac{1}{p^{\prime}}\right)}\|f\|_{W^{k, p}(\mathbb{R})}
$$

for $0 \leq k \leq 2$ and $1<p \leq 2$.

Proof. The case $k=0$ is obtained by interpolating between Lemma 6.11 and Proposition 7.1 and holds for the entire range $1 \leq p \leq 2$. We need to require $p>1$ only for the derivatives. If $a$ is sufficiently large, then

$$
(\mathcal{H}-i a)^{-1}: L^{2} \times L^{2} \rightarrow W^{2,2} \times W^{2,2}
$$

is an isomorphism. More generally,

$$
(\mathcal{H}-i a)^{-\frac{1}{2}}: L^{p} \times L^{p} \rightarrow W^{2, p} \times W^{2, p}
$$


is an isomorphism for $1<p<\infty$. This can be seen from the resolvent identity

$$
(\mathcal{H}-i a)^{-1}=\left(\mathcal{H}_{0}-i a\right)^{-1}\left[1+V\left(\mathcal{H}_{0}-i a\right)^{-1}\right]^{-1},
$$

since $\|V\|_{\infty}<\infty$ implies that

$$
\left\|V\left(\mathcal{H}_{0}-i a\right)^{-1}\right\|_{p \rightarrow p}<\frac{1}{2}
$$

if $a$ is large enough and because

$$
\left(\mathcal{H}_{0}-i a\right)^{-\frac{1}{2}}: L^{p} \times L^{p} \rightarrow W^{2, p} \times W^{2, p}
$$

for any $a \neq 0$ as an isomorphism. Hence,

$$
\begin{aligned}
\left\|\partial_{x}^{2} e^{i t \mathcal{H}} P_{s} f\right\|_{p^{\prime}} & \leq C\left\|(\mathcal{H}-i a) e^{i t \mathcal{H}} f\right\|_{p^{\prime}}=\left\|e^{i t \mathcal{H}}(\mathcal{H}-i a) f\right\|_{p^{\prime}} \\
& \leq C t^{-\frac{1}{2}\left(\frac{1}{p}-\frac{1}{p^{\prime}}\right)}\|(\mathcal{H}-i a) f\|_{p} \leq C t^{-\frac{1}{2}\left(\frac{1}{p}-\frac{1}{p^{\prime}}\right)}\|f\|_{W^{2, p}(\mathbb{R})} .
\end{aligned}
$$

This gives the case $k=2$ of the lemma, whereas $k=1$ follows by interpolating between $k=0$ and $k=2$.

Finally, as in [44] one can now derive Strichartz estimates on the evolution $e^{i t \mathcal{H}} P_{s}$, even with derivatives.

Corollary 7.3. Let $\mathcal{H}$ be admissible. Then the following Strichartz estimates hold:

$$
\begin{aligned}
\left\|e^{-i t \mathcal{H}} P_{s} f\right\|_{L_{t}^{r}\left(W_{x}^{k, p}\right)} & \leq C\|f\|_{W^{k, 2}}, \\
\left\|\int_{0}^{t} e^{-i(t-s) \mathcal{H}} P_{s} F(s) d s\right\|_{L_{t}^{r}\left(W_{x}^{k, p}\right)} & \leq C\|F\|_{L_{t}^{a^{\prime}}\left(W_{x}^{k, b^{\prime}}\right)},
\end{aligned}
$$

provided $(r, p),(a, b)$ are admissible, i.e., $4<r \leq \infty$ and $\frac{2}{r}+\frac{1}{p}=\frac{1}{2}$ and the same for $(a, b)$. Here $k$ is an integer, $0 \leq k \leq 2$.

Proof. We first show how to reduce matters to $k=0$. As in the previous proof, we rely on the fact that (because of $\|V\|_{\infty}<\infty$ ),

$$
\left\|\partial_{x}^{2} f\right\|_{q} \leq C\|(\mathcal{H}-i a) f\|_{q}
$$

for any $1<q<\infty$. Hence,

$$
\begin{aligned}
\left\|e^{-i t \mathcal{H}} P_{s} f\right\|_{L_{t}^{r}\left(W_{x}^{2, p}\right)} & \leq C\left\|(\mathcal{H}-i a) e^{-i t \mathcal{H}} P_{s} f\right\|_{L_{t}^{r}\left(L_{x}^{p}\right)}=\left\|e^{-i t \mathcal{H}} P_{s}(\mathcal{H}-i a) f\right\|_{L_{t}^{r}\left(L_{x}^{p}\right)} \\
& \leq C\|(\mathcal{H}-i a) f\|_{2} \leq C\|f\|_{W^{2,2}},
\end{aligned}
$$

which is (7.22) for $k=2$. Similarly, one proves (7.23) for $k=2$. The case $k=1$ is then obtained by interpolation between $k=2$ and $k=0$. For $k=0$ we use an argument from 41, Section 4. Let ( $\mathcal{S}$ for "Strichartz")

$$
(\mathcal{S} F)(t, x)=\int_{0}^{t}\left(e^{-i(t-s) \mathcal{H}} P_{s} F(s, \cdot)\right)(x) d s .
$$

In this proof it will be understood that all times are $\geq 0$. Then by (6.14),

$$
\|\mathcal{S} F\|_{L_{t}^{\infty}\left(L_{x}^{2}\right)} \leq C\|F\|_{L_{t}^{1}\left(L_{x}^{2}\right)},
$$

and more generally, by the usual fractional integration argument based on Proposition 7.1

$$
\|\mathcal{S} F\|_{L_{t}^{r}\left(L_{x}^{p}\right)} \leq C\|F\|_{L_{t}^{r^{\prime}}\left(L_{x}^{p^{\prime}}\right)}
$$

for any admissible pair $(r, p)$. In the unitary case this implies (7.22) via a $T T^{*}$ argument, but this reasoning does not apply here. Instead, we rely on a Kato 
theory type approach as in 41, Section 4 . Since $\mathcal{H}=\mathcal{H}_{0}+V$, Duhamel's formula yields

$$
e^{-i t \mathcal{H}} P_{s}=e^{-i t \mathcal{H}_{0}} P_{s}-i \int_{0}^{t} e^{-i(t-s) \mathcal{H}_{0}} V e^{-i s \mathcal{H}} P_{s} d s
$$

Set $\rho(x)=\langle x\rangle^{-1-}$, say, and define

$$
\tilde{M}=\left(\begin{array}{cc}
\rho & 0 \\
0 & \rho
\end{array}\right) .
$$

With $V=\tilde{M} \tilde{M}^{-1} V$, observe first that

$$
\left\|\int_{0}^{\infty} e^{-i(t-s) \mathcal{H}_{0}} \tilde{M} g(s) d s\right\|_{L_{t}^{r}\left(L_{x}^{p}\right)} \lesssim\left\|\int_{0}^{\infty} e^{i s \mathcal{H}_{0}} \tilde{M} g(s) d s\right\|_{L^{2}} \leq C\|g\|_{L_{s}^{2}\left(L_{x}^{2}\right)},
$$

where the last inequality is the dual of the smoothing bound

$$
\int_{0}^{\infty}\left\|\tilde{M} e^{-i s \mathcal{H}_{0}^{*}} \psi\right\|_{2}^{2} d s \leq C\|\psi\|_{2}^{2} .
$$

Here "smoothing" is used in the sense of Kato's theory; see [28] and [40]. Now one applies the Christ-Kiselev lemma, see [8] or [41, to conclude that

$$
\left\|\int_{0}^{t} e^{-i(t-s) \mathcal{H}_{0}} \tilde{M} g(s) d s\right\|_{L_{t}^{r}\left(L_{x}^{p}\right)} \leq C\|g\|_{L_{s}^{2}\left(L_{x}^{2}\right)}
$$

for any admissible pair $(r, p)$. Hence, continuing in (7.25), one obtains (using that $\left.\left\|P_{s} f\right\|_{2} \leq C\|f\|_{2}\right)$

$$
\left\|e^{-i t \mathcal{H}} P_{s} f\right\|_{L_{t}^{r}\left(L_{x}^{p}\right)} \leq C\|f\|_{2}+\left\|\tilde{M}^{-1} V e^{-i s \mathcal{H}} P_{s} f\right\|_{L_{s}^{2}\left(L_{x}^{2}\right)} .
$$

It remains to show that $\tilde{M}^{-1} V$ is $\mathcal{H} P_{s}$-smoothing, i.e.,

$$
\left\|\tilde{M}^{-1} V e^{-i s \mathcal{H}} P_{s} f\right\|_{L_{s}^{2}\left(L_{x}^{2}\right)} \leq C\|f\|_{2} .
$$

It follows from Lemma 6.8 that the integrand here is the same as

$$
\tilde{M}^{-1} V e^{-i s \mathcal{H}} P_{s} f=\frac{1}{2 \pi i} \int_{|\lambda| \geq 1} e^{i t \lambda} \tilde{M}^{-1} V\left[(\mathcal{H}-(\lambda+i 0))^{-1}-(\mathcal{H}-(\lambda-i 0))^{-1}\right] f d \lambda .
$$

Hence, applying Plancherel in $t$ yields

$$
\begin{aligned}
& \int_{-\infty}^{\infty}\left\|\tilde{M}^{-1} V e^{-i s \mathcal{H}} P_{s} f\right\|_{2}^{2} d s \\
& =\int_{|\lambda| \geq 1}\left\|\tilde{M}^{-1} V\left[(\mathcal{H}-(\lambda+i 0))^{-1}-(\mathcal{H}-(\lambda-i 0))^{-1}\right] f\right\|_{2}^{2} d \lambda \\
& =2 \int_{0}^{\infty} \lambda\left\|\tilde{M}^{-1} V\left[\left(\mathcal{H}-\left(\lambda^{2}+1+i 0\right)\right)^{-1}-\left(\mathcal{H}-\left(\lambda^{2}+1-i 0\right)\right)^{-1}\right] f\right\|_{2}^{2} d \lambda \\
& \quad+2 \int_{0}^{\infty} \lambda\left\|\tilde{M}^{-1} V\left[\left(\mathcal{H}-\left(-\lambda^{2}+1+i 0\right)\right)^{-1}-\left(\mathcal{H}-\left(-\lambda^{2}+1-i 0\right)\right)^{-1}\right] f\right\|_{2}^{2} d \lambda
\end{aligned}
$$


In view of Lemma 6.7 .

$$
\begin{aligned}
& \int_{-\infty}^{\infty}\left\|\tilde{M}^{-1} V e^{-i s \mathcal{H}} P_{s} f\right\|_{2}^{2} d s \\
& =\frac{1}{2} \int_{0}^{\infty} \lambda^{-1}\left\|\tilde{M}^{-1} V \mathcal{E}_{+}(x, \lambda)\left\langle\mathcal{E}_{+}^{*}(\cdot, \lambda) \sigma_{3}, f\right\rangle\right\|_{L_{x}^{2}}^{2} d \lambda \\
& \quad+\frac{1}{2} \int_{0}^{\infty} \lambda^{-1}\left\|\tilde{M}^{-1} V \mathcal{E}_{-}(x, \lambda)\left\langle\mathcal{E}_{-}^{*}(\cdot, \lambda) \sigma_{3}, f\right\rangle\right\|_{L_{x}^{2}}^{2} d \lambda
\end{aligned}
$$

By the definition of $\mathcal{E}$ and Lemma 6.3 .

$$
\left\|\tilde{M}^{-1} V \mathcal{E}_{ \pm}(x, \lambda)\right\|_{L_{x}^{2}}^{2} \leq C|\lambda|^{2}(1+|\lambda|)^{-2}
$$

Hence,

$$
\begin{aligned}
& \frac{1}{2} \int_{0}^{\infty} \lambda^{-1}\left\|\tilde{M}^{-1} V \mathcal{E}_{+}(x, \lambda)\left\langle\mathcal{E}_{+}^{*}(\cdot, \lambda) \sigma_{3}, f\right\rangle\right\|_{L_{x}^{2}}^{2} d \lambda \\
& +\frac{1}{2} \int_{0}^{\infty} \lambda^{-1}\left\|\tilde{M}^{-1} V \mathcal{E}_{-}(x, \lambda)\left\langle\mathcal{E}_{-}^{*}(\cdot, \lambda) \sigma_{3}, f\right\rangle\right\|_{L_{x}^{2}}^{2} d \lambda \\
& \leq C \int_{0}^{\infty}\left|\left\langle\mathcal{E}_{+}^{*}(\cdot, \lambda) \sigma_{3}, f\right\rangle\right|_{L_{x}^{2}}^{2} d \lambda+C \int_{0}^{\infty}\left|\left\langle\mathcal{E}_{-}^{*}(\cdot, \lambda) \sigma_{3}, f\right\rangle\right|_{L_{x}^{2}}^{2} d \lambda \leq C\|f\|_{2}^{2},
\end{aligned}
$$

where the final inequality is a Plancherel type property that follows from Lemma 6.3 . Hence (7.27) holds. The conclusion is that

$$
\left\|e^{-i t \mathcal{H}} P_{s} f\right\|_{L_{t}^{r}\left(L_{x}^{p}\right)} \leq C\|f\|_{2}
$$

for any admissible $(r, p)$, which is (7.22). The proof of (7.23) is now the usual interpolation argument. Indeed, in view of the preceding, one has the following bounds on $\mathcal{S}$ for any admissible pair $(r, p)$ :

$$
\begin{aligned}
& \mathcal{S}: L_{t}^{1}\left(L_{x}^{2}\right) \rightarrow L_{t}^{r}\left(L_{x}^{p}\right), \\
& \mathcal{S}: L_{t}^{r^{\prime}}\left(L_{x}^{p^{\prime}}\right) \rightarrow L_{t}^{r}\left(L_{x}^{p}\right), \\
& \mathcal{S}: L_{t}^{r^{\prime}}\left(L_{x}^{p^{\prime}}\right) \rightarrow L_{t}^{\infty}\left(L_{x}^{2}\right) .
\end{aligned}
$$

These estimates arise as follows: (7.29) is exactly (7.24), whereas (7.28) follows from (7.22) by means of Minkowski's inequality. Finally, (7.30) is dual to the bound

$$
\left\|\int_{t}^{\infty} e^{i(t-s) \mathcal{H}^{*}} \tilde{P}_{c} G(s) d s\right\|_{L_{t}^{r}\left(L_{x}^{p}\right)} \lesssim\|G\|_{L_{t}^{1}\left(L_{x}^{2}\right)} .
$$

Here $\tilde{P}_{c}$ corresponds to $\mathcal{H}^{*}$ in the same way that $P_{s}$ corresponds to $\mathcal{H}$. In particular, one has

$$
\left\|e^{-i t \mathcal{H}^{*}} \tilde{P}_{c}\right\|_{1 \rightarrow \infty} \leq C t^{-\frac{1}{2}}
$$

and therefore, (7.31) is derived be the same methods as (7.28). It is important to notice that $P_{s}^{*}=\tilde{P}_{s}$ which is essential for the duality argument here. This can be seen, for example, by writing the Riesz projections onto (generalized) eigenspaces as contour integrals around circles surrounding the eigenvalues. Since the (complex) eigenvalues always come in pairs, the adjoints have the desired property. Interpolating between (7.28) and (7.29) yields (7.23) for the range $a^{\prime} \leq r^{\prime}$ or $a \geq r$, whereas interpolating between (7.28) and (7.29) yields (7.23) in the range $a \leq r$. 


\section{Dispersive estimates: The Weighted Case}

In this section we obtain the decay rate of $t^{-\frac{3}{2}}$ on $e^{i t \mathcal{H}} P_{s}$. The latter will exploit the fact that absence of resonances at the thresholds leads to better time-decay, albeit at the cost of a linear weight. This property was discovered by Murata 34 . and then used by Buslaev and Perelman [5] for systems. However, they worked on $L^{2}$ and with a loss of a higher power $x$, namely of $x^{3.5+\varepsilon}$. This would not be sufficient for our purposes. For the same estimate in the scalar case, see [45].

Proposition 8.1. Let $\mathcal{H}$ be admissible; see Definition 6.1. Then for all $t \neq 0$,

$$
\left\|\langle x\rangle^{-1} e^{i t \mathcal{H}} P_{s} f\right\|_{\infty} \leq C|t|^{-\frac{3}{2}}\|\langle x\rangle f\|_{1}
$$

with some $C=C(V)$.

Proof. As in the proof of Proposition 7.1, we divide the arguments into $\lambda$ large and not large. In the former case, the estimates that lead to the unweighted $L^{1} \rightarrow L^{\infty}$ bound apply here almost verbatim up to an additional integration by parts, whereas in the latter case we will need to use the Fourier representation from Proposition 6.9. As before, we start from the representation formula

$$
\begin{aligned}
& \left\langle e^{i t \mathcal{H}} P_{s} \phi, \psi\right\rangle \\
& =\frac{e^{i t}}{\pi i} \int_{0}^{\infty} e^{i t \lambda^{2}} \lambda\left\langle\left(\left(\mathcal{H}-\left(\lambda^{2}+1+i 0\right)\right)^{-1}-\left(\mathcal{H}-\left(\lambda^{2}+1-i 0\right)\right)^{-1}\right) \phi, \psi\right\rangle d \lambda \\
& +\frac{e^{-i t}}{\pi i} \int_{0}^{\infty} e^{-i t \lambda^{2}} \lambda\left\langle\left(\left(\mathcal{H}-\left(-\lambda^{2}-1+i 0\right)\right)^{-1}-\left(\mathcal{H}-\left(-\lambda^{2}-1-i 0\right)\right)^{-1}\right) \phi, \psi\right\rangle d \lambda,
\end{aligned}
$$

which holds in the principal value sense if $\phi, \psi \in \mathcal{S}$. With the same $\chi$ as in the proof of Proposition 7.1, we arrive at

$$
\begin{aligned}
&\left|\left\langle e^{i t \mathcal{H}}(1-\chi(H-I)) P_{s}^{+} \phi, \psi\right\rangle\right| \\
& \leq C \sum_{n=0}^{\infty}|t|^{-1} \mid \int_{0}^{\infty} e^{i t \lambda^{2}} \frac{d}{d \lambda}\left\{( 1 - \chi ( \lambda ^ { 2 } ) ) \left[\left\langle\left(\mathcal{H}_{0}-\left(\lambda^{2}+1+i 0\right)\right)^{-1}\right.\right.\right. \\
&\left.\quad \times\left(V\left(\mathcal{H}_{0}-\left(\lambda^{2}+1+i 0\right)\right)^{-1}\right)^{n} \phi, \psi\right\rangle \\
&\left.\left.\quad-\left\langle\left(\mathcal{H}_{0}-\left(\lambda^{2}+1-i 0\right)\right)^{-1}\left(V\left(\mathcal{H}_{0}-\left(\lambda^{2}+1-i 0\right)\right)^{-1}\right)^{n} \phi, \psi\right\rangle\right]\right\} d \lambda \mid
\end{aligned}
$$

If the $\lambda$-derivative falls on $1-\chi\left(\lambda^{2}\right)$, then the exact same arguments apply which we used in the unweighted case. If the derivative falls on the resolvents, then weights $\left|x_{j}-x_{j-1}\right|$ appear. However, these are bounded by $\left|x_{j}\right|+\left|x_{j-1}\right|$ and can therefore be absorbed either by $V$ or the test functions $\phi, \psi$. The conclusion is that

$$
\left|\left\langle e^{i t \mathcal{H}}(1-\chi(H-I)) P_{s}^{+} \phi, \psi\right\rangle\right| \leq C|t|^{-\frac{3}{2}}\|\langle x\rangle \phi\|_{1}\|\langle x\rangle \psi\|_{1},
$$

and therefore also

$$
\left|\left\langle e^{i t \mathcal{H}}(1-\chi(-H+I)) P_{s}^{-} \phi, \psi\right\rangle\right| \leq C|t|^{-\frac{3}{2}}\|\langle x\rangle \phi\|_{1}\|\langle x\rangle \psi\|_{1},
$$

as desired. Cf. the treatment of (7.3) $-(7.5)$ for details.

Next, we use Proposition 6.9 to write

$$
\begin{aligned}
\left\langle e^{i t \mathcal{H}} \chi(H-I) P_{s}^{+} \phi, \psi\right\rangle & =\frac{e^{i t}}{2 \pi} \int_{-\infty}^{\infty} e^{i t \lambda^{2}} \chi\left(\lambda^{2}\right)\left\langle\phi, \sigma_{3} e_{+}(., \lambda)\right\rangle\left\langle e_{+}(\cdot, \lambda), \psi\right\rangle d \lambda \\
\left\langle e^{i t \mathcal{H}} \chi(-H+I) P_{s}^{-} \phi, \psi\right\rangle & =\frac{e^{-i t}}{2 \pi} \int_{-\infty}^{\infty} e^{i t \lambda^{2}} \chi\left(\lambda^{2}\right)\left\langle\phi, \sigma_{3} e_{-}(., \lambda)\right\rangle\left\langle e_{-}(\cdot, \lambda), \psi\right\rangle d \lambda .
\end{aligned}
$$


It suffices to estimate the first integral. Integrating by parts in $\lambda$ yields

$$
\left\langle e^{i t \mathcal{H}} \chi(H-I) P_{s}^{+} \phi, \psi\right\rangle
$$

$$
\begin{aligned}
= & \frac{1}{\pi i t} \int_{0}^{\infty} e^{i t \lambda^{2}} \partial_{\lambda}\left[\chi\left(\lambda^{2}\right) \lambda\left\langle\phi, \sigma_{3} F_{1}(\cdot, \lambda) D^{-1}(\lambda) \underline{e}\right\rangle\left\langle F_{1}(\cdot, \lambda) D^{-1}(\lambda) \underline{e}, \psi\right\rangle\right] d \lambda \\
& +\frac{1}{\pi i t} \int_{-\infty}^{0} e^{i t \lambda^{2}} \partial_{\lambda}\left[\chi\left(\lambda^{2}\right) \lambda\left\langle\phi, \sigma_{3} G_{2}(\cdot,-\lambda) D^{-1}(\lambda) \underline{e}\right\rangle\left\langle G_{2}(\cdot,-\lambda) D^{-1}(\lambda) \underline{e}, \psi\right\rangle\right] d \lambda .
\end{aligned}
$$

By symmetry, it will suffice to treat the integral (8.1) involving $F_{1}(\cdot, \lambda)$. We distinguish three cases, depending on where the derivative $\partial_{\lambda}$ falls. We start with the integral

$$
\int_{0}^{\infty} e^{i t \lambda^{2}} \omega(\lambda)\left\langle\phi, \sigma_{3} F_{1}(\cdot, \lambda) D^{-1}(\lambda) \underline{e}\right\rangle\left\langle F_{1}(\cdot, \lambda) D^{-1}(\lambda) \underline{e}, \psi\right\rangle d \lambda,
$$

where we have set $\omega(\lambda)=\partial_{\lambda}\left[\chi\left(\lambda^{2}\right) \lambda\right]$. By the preceding, $\omega$ is a smooth function with compact support in $[0, \infty)$. As usual, we will estimate (8.2) by means of a Fourier transform in $\lambda$. Since we are working on a half-line, this will actually be a cosine transform. Let $\tilde{\omega}$ be another cut-off function satisfying $\omega \tilde{\omega}=\omega$. Then

$$
\begin{aligned}
& \left|\int_{0}^{\infty} e^{i t \lambda^{2}} \omega(\lambda)\left\langle\phi, \sigma_{3} F_{1}(\cdot, \lambda) D^{-1}(\lambda) \underline{e}\right\rangle\left\langle F_{1}(\cdot, \lambda) D^{-1}(\lambda) \underline{e}, \psi\right\rangle d \lambda\right| \\
& \leq C|t|^{-\frac{1}{2}}\left\|\left[\omega\left\langle\phi, \sigma_{3} F_{1}(\cdot, \lambda) D^{-1}(\lambda) \underline{e}\right\rangle\right]^{\vee}\right\|_{1}\left\|\left[\tilde{\omega}\left\langle F_{1}(\cdot, \lambda) D^{-1}(\lambda) \underline{e}, \psi\right\rangle\right]^{\vee}\right\|_{1} .
\end{aligned}
$$

It remains to show that

$$
\left[\omega\left\langle\phi, \sigma_{3} F_{1}(\cdot, \lambda) D^{-1}(\lambda) \underline{e}\right\rangle\right]^{\vee}(u):=\int_{0}^{\infty} \cos (u \lambda) \omega(\lambda)\left\langle\phi, \sigma_{3} F_{1}(\cdot, \lambda) D^{-1}(\lambda) \underline{e}\right\rangle d \lambda
$$

satisfies

$$
\int_{-\infty}^{\infty}\left|\left[\omega\left\langle\phi, \sigma_{3} F_{1}(\cdot, \lambda) D^{-1}(\lambda) \underline{e}\right\rangle\right]^{\vee}(u)\right| d u \leq C\|\langle x\rangle \phi\|_{1} .
$$

The second $L^{1}$-norm in (8.3) is treated the same way. This means that we need to prove that

$$
\int_{-\infty}^{\infty}\left|\left[\omega F_{1}(x, \lambda) D^{-1}(\lambda) \underline{e}\right]^{\vee}(u)\right| d u \leq C\langle x\rangle
$$

for all $x \in \mathbb{R}$. We will consider the cases $x \geq 0$ and $x \leq 0$ separately. In the former case,

$$
\begin{aligned}
& {\left[\omega F_{1}(x, \cdot) D^{-1}(\lambda) \underline{e}\right]^{\vee}(u):=\int_{0}^{\infty} \cos (u \lambda) \omega(\lambda) F_{1}(x, \lambda) D^{-1}(\lambda) \underline{e} d \lambda} \\
& =\frac{1}{2} \int_{0}^{\infty} e^{i(x+u) \lambda} \omega(\lambda) e^{-i x \lambda} f_{1}(x, \lambda)\left\langle\underline{e}, D^{-1}(\lambda) \underline{e}\right\rangle d \lambda \\
& +\frac{1}{2} \int_{0}^{\infty} e^{i(x-u) \lambda} \omega(\lambda) e^{-i x \lambda} f_{1}(x, \lambda)\left\langle\underline{e}, D^{-1}(\lambda) \underline{e}\right\rangle d \lambda \\
& +\int_{0}^{\infty} \cos (u \lambda) \omega(\lambda) e^{-\mu x} e^{\mu x} f_{3}(x, \lambda)\left\langle\underline{e^{\prime}}, D^{-1}(\lambda) \underline{e}\right\rangle d \lambda,
\end{aligned}
$$


where $\underline{e}^{\prime}=\left(\begin{array}{l}0 \\ 1\end{array}\right)$. We integrate by parts in $(\underline{8.5})$ (8.7):

$$
\text { (8.5) }+8.6
$$

$$
\begin{aligned}
= & -\frac{1}{2 i(x+u)} \omega(0) f_{1}(x, 0)\left\langle\underline{e}, D^{-1}(0) \underline{e}\right\rangle-\frac{1}{2 i(x-u)} \omega(0) f_{1}(x, 0)\left\langle\underline{e}, D^{-1}(0) \underline{e}\right\rangle \\
& -\frac{1}{2 i(x+u)} \int_{0}^{\infty} e^{i(x+u) \lambda} \partial_{\lambda}\left[\omega(\lambda) e^{-i x \lambda} f_{1}(x, \lambda)\left\langle\underline{e}, D^{-1}(\lambda) \underline{e}\right\rangle\right] d \lambda \\
& -\frac{1}{2 i(x-u)} \int_{0}^{\infty} e^{i(x-u) \lambda} \partial_{\lambda}\left[\omega(\lambda) e^{-i x \lambda} f_{1}(x, \lambda)\left\langle\underline{e} D^{-1}(\lambda) \underline{e}\right\rangle\right] d \lambda,
\end{aligned}
$$

whereas

$$
\begin{aligned}
& \int_{0}^{\infty} \cos (u \lambda) \omega(\lambda) e^{-\mu x} e^{\mu x} f_{3}(x, \lambda)\left\langle\underline{e}^{\prime}, D^{-1}(\lambda) \underline{e}\right\rangle d \lambda \\
& =-u^{-1} \int_{0}^{\infty} \sin (\lambda u) \partial_{\lambda}\left[\omega(\lambda) e^{-\mu x} e^{\mu x} f_{3}(x, \lambda)\left\langle\underline{e}^{\prime}, D^{-1}(\lambda) \underline{e}\right\rangle\right] d \lambda .
\end{aligned}
$$

Since

$$
\sup _{x \geq 0, \lambda}\left|\partial_{\lambda}^{j}\left[\omega(\lambda) e^{-i x \lambda} f_{1}(x, \lambda)\right]\right| \leq C(V), \sup _{x \geq 0, \lambda}\left|\partial_{\lambda}^{j}\left[\omega(\lambda) e^{x \mu} f_{3}(x, \lambda)\right]\right| \leq C(V),
$$

as well as

$$
\sup _{x \geq 0}\left|\partial_{\lambda}^{j}\left[\omega(\lambda) e^{-\mu x}\right]\right| \leq C
$$

for $j \geq 0$, it follows via another integration by parts that

$$
\left|\left[\omega F_{1}(x, \cdot) D^{-1}(\lambda) \underline{e}\right]^{\vee}(u)\right| \leq C \frac{|x|}{\left|x^{2}-u^{2}\right|}+C(u+x)^{-2}+C(u-x)^{-2}+C u^{-2} .
$$

We will use this bound if ||$u|-| x||>|x|$ and $|u| \geq 1$. On the other hand, if ||$u|-| x|| \leq|x|$, or $|u| \leq 1$, then we simply estimate

$$
\left|\left[\omega F_{1}(x, \cdot) D^{-1}(\lambda) \underline{e}\right]^{\vee}(u)\right| \leq C .
$$

The conclusion is that

$$
\int_{\mathbb{R}}\left|\left[\omega(\lambda) F_{1}(x, \lambda) D^{-1}(\lambda) \underline{e}\right]^{\vee}(u)\right| d u \leq C\langle x\rangle
$$

for all $x \geq 0$.

If $x \leq 0$, then recall that

$$
\begin{aligned}
& 2 i \lambda F_{1}(x, \lambda) D^{-1}(\lambda) \underline{e} \\
& =2 i \lambda G_{1}(x, \lambda) A(\lambda) D^{-1}(\lambda) \underline{e}+2 i \lambda G_{2}(x, \lambda) B(\lambda) D^{-1}(\lambda) \underline{e} \\
& =G_{1}(x, \lambda)\left(\begin{array}{cc}
1 & 0 \\
0 & -i \frac{\lambda}{\mu}
\end{array}\right) \underline{e}+G_{2}(x, \lambda) \underline{e}\left\langle 2 i \lambda p B(\lambda) D^{-1}(\lambda) \underline{e}, \underline{e}\right\rangle \\
& \quad \quad+G_{2}(x, \lambda) \underline{e}^{\prime}\left\langle 2 i \lambda q B(\lambda) D^{-1}(\lambda) \underline{e}, \underline{e}^{\prime}\right\rangle \\
& (8.12) \\
& =f_{2}(-x, \lambda)+r(\lambda) f_{1}(-x, \lambda)+f_{3}(-x, \lambda)\left\langle 2 i \lambda q B(\lambda) D^{-1}(\lambda) \underline{e}, \underline{e}^{\prime}\right\rangle \\
& =f_{1}(-x,-\lambda)-f_{1}(-x, \lambda)+(r(\lambda)+1) f_{1}(-x, \lambda)+f_{3}(-x, \lambda)\left\langle 2 i \lambda q B(\lambda) D^{-1}(\lambda) \underline{e}, \underline{e}^{\prime}\right\rangle,
\end{aligned}
$$


where $r(\lambda)$ is as in Lemma 6.3 Thus,

$$
\begin{aligned}
& F_{1}(x, \lambda) D^{-1}(\lambda) \underline{e} \\
&= 2 i \lambda G_{1}(x, \lambda) A(\lambda) D^{-1}(\lambda) \underline{e}+2 i \lambda G_{2}(x, \lambda) B(\lambda) D^{-1}(\lambda) \underline{e} \\
&=(2 i \lambda)^{-1}\left[f_{1}(-x,-\lambda)-f_{1}(-x, \lambda)\right]+\frac{r(\lambda)+1}{2 i \lambda} f_{1}(-x, \lambda) \\
&+f_{3}(-x, \lambda)\left\langle q B(\lambda) D^{-1}(\lambda) \underline{e}, \underline{e}^{\prime}\right\rangle .
\end{aligned}
$$

By Corollary 5.15 and Lemma 6.2, $\left\langle q B(\lambda) D^{-1}(\lambda) \underline{e}, \underline{e}^{\prime}\right\rangle$ is smooth in $\lambda$. Returning to the cosine transform (where $x=-y$ with $y \geq 0$ ), we conclude that

$$
\begin{aligned}
& \int_{0}^{\infty} \cos (u \lambda) \omega(\lambda) F_{1}(-y, \lambda) D^{-1}(\lambda) \underline{e} d \lambda \\
& =\int_{0}^{\infty} \cos (\lambda u) \omega(\lambda)(2 i \lambda)^{-1}\left[f_{1}(y,-\lambda)-f_{1}(y, \lambda)\right] d \lambda \\
& \quad+\int_{0}^{\infty} \cos (\lambda u) e^{i y \lambda} \omega(\lambda) \frac{r(\lambda)+1}{2 i \lambda} e^{-i y \lambda} f_{1}(y, \lambda) d \lambda \\
& \quad+\int_{0}^{\infty} \cos (\lambda u) e^{-\mu y}\left\langle q B(\lambda) D^{-1}(\lambda) \underline{e}, \underline{e}^{\prime}\right\rangle e^{\mu y} f_{3}(y, \lambda) d \lambda .
\end{aligned}
$$

The easiest term to deal with is (8.15). Indeed, integrating by parts in $\lambda$ twice shows that

$$
\sup _{y \geq 0}\left|\int_{0}^{\infty} \cos (\lambda u) e^{-\mu y}\left\langle q B(\lambda) D^{-1}(\lambda) \underline{e}, \underline{e}^{\prime}\right\rangle e^{\mu y} f_{3}(y, \lambda) d \lambda\right| \leq C(1+|u|)^{-2} ;
$$

see (8.9) and (8.10). Next, consider (8.14). By Lemma 6.3, $r(0)=-1$ and $r(\lambda)$ is smooth. Hence, $\frac{r(\lambda)+1}{\lambda}$ is also a smooth function. Set $\omega_{1}(\lambda)=\omega(\lambda) \frac{r(\lambda)+1}{\lambda}$. Then

$$
\text { (8.14) }=\int_{0}^{\infty} \cos (u \lambda) e^{i y \lambda} \omega_{1}(\lambda) e^{-i y \lambda} f_{1}(y, \lambda) d \lambda .
$$

By the same arguments that lead from (8.8) to (8.11) we obtain the bound

$$
\int_{-\infty}^{\infty}\left|\int_{0}^{\infty} \cos (u \lambda) e^{i y \lambda} \omega_{1}(\lambda) e^{-i y \lambda} f_{1}(y, \lambda) d \lambda\right| d u \leq C\langle y\rangle
$$

uniformly in $y \geq 0$. Turning to (8.13), we see that is the same as (with $\partial_{2}$ being the partial derivative with respect to the second variable of $f_{1}$ and ignoring constants)

$$
\begin{aligned}
& \int_{-1}^{1} \int_{0}^{\infty} \cos (u \lambda) \omega(\lambda) \partial_{2} f_{1}(x, \lambda \sigma) d \lambda d \sigma \\
& =\int_{-1}^{1} \int_{0}^{\infty} \cos (u \lambda) e^{i \lambda x \sigma} \omega(\lambda) \partial_{2}\left[e^{-i \lambda x \sigma} f_{1}(x, \lambda \sigma)\right] d \lambda d \sigma \\
& \quad+i x \int_{-1}^{1} \int_{0}^{\infty} \cos (u \lambda) e^{i \lambda x \sigma} \omega(\lambda) e^{-i \lambda x \sigma} f_{1}(x, \lambda \sigma) d \lambda d \sigma .
\end{aligned}
$$


We will focus on the second integral (8.19), since the first one (8.18) is similar. We will integrate by parts in $\lambda$, but only on the set $|\sigma x \pm u| \geq 1$. Then

$$
\begin{aligned}
& -i x \int_{-1}^{1} \int_{0}^{\infty} \cos (u \lambda) e^{i \lambda x \sigma} \omega(\lambda) e^{-i \lambda x \sigma} f_{1}(x, \lambda \sigma) d \lambda \chi_{[|\sigma x \pm u| \geq 1]} d \sigma \\
& =\int_{-1}^{1} \frac{x}{2(\sigma x+u)} \omega(0) f_{1}(x, 0) \chi_{[|\sigma x \pm u| \geq 1]} d \sigma \\
& +\int_{-1}^{1} \frac{x}{2(\sigma x-u)} \omega(0) f_{1}(x, 0) \chi_{[|\sigma x \pm u| \geq 1]} d \sigma \\
& +\int_{-1}^{1} \frac{x}{2(\sigma x+u)} \int_{0}^{\infty} e^{i(\sigma x+u) \lambda} \partial_{\lambda}\left[\omega(\lambda) e^{-i x \lambda \sigma} f_{1}(x, \lambda)\right] d \lambda \chi_{[|\sigma x \pm u| \geq 1]} d \sigma \\
& +\int_{-1}^{1} \frac{x}{2(\sigma x-u)} \int_{0}^{\infty} e^{i(\sigma x-u) \lambda} \partial_{\lambda}\left[\omega(\lambda) e^{-i x \lambda \sigma} f_{1}(x, \lambda)\right] d \lambda \chi_{[|\sigma x \pm u| \geq 1]} d \sigma .
\end{aligned}
$$

The first two integrals here (which are due to the boundary $\lambda=0$ ) contribute

$$
\begin{aligned}
& \int_{-1}^{1} \frac{x}{2(\sigma x+u)} \omega(0) f_{1}(x, 0) \chi_{[|\sigma x \pm u| \geq 1]} d \sigma \\
& +\int_{-1}^{1} \frac{x}{2(-\sigma x-u)} \omega(0) f_{1}(x, 0) \chi_{[|\sigma x \pm u| \geq 1]} d \sigma=0,
\end{aligned}
$$

where we performed a change of variables $\sigma \mapsto-\sigma$ in the second one. Integrating by parts one more time in (8.20) and (8.21) with respect to $\lambda$ implies

$$
\begin{aligned}
& \int_{-\infty}^{\infty}\left|x \int_{-1}^{1} \int_{0}^{\infty} \cos (u \lambda) e^{i \lambda x \sigma} \omega(\lambda) e^{-i \lambda x \sigma} f_{1}(x, \lambda \sigma) d \lambda \chi_{[|\sigma x \pm u| \geq 1]} d \sigma\right| d u \\
& \leq C \int_{-\infty}^{\infty} \int_{-1}^{1} \frac{|x|}{(\sigma x+u)^{2}} \chi_{[|\sigma x \pm u| \geq 1]} d \sigma d u \\
& +C \int_{-\infty}^{\infty} \int_{-1}^{1} \frac{|x|}{(\sigma x-u)^{2}} \chi_{[|\sigma x \pm u| \geq 1]} d \sigma d u \leq C|x| .
\end{aligned}
$$

Finally, the cases $|\sigma x+u| \leq 1$ and $|\sigma x-u| \leq 1$ each contribute at most $C|x|$ to the $u$-integral. Hence

$$
\int_{-\infty}^{\infty}\left|x \int_{-1}^{1} \int_{0}^{\infty} \cos (u \lambda) e^{i \lambda x \sigma} \omega(\lambda) e^{-i \lambda x \sigma} f_{1}(x, \lambda \sigma) d \lambda d \sigma\right| d u \leq C|x| .
$$

Since (8.18) can be treated the same way (in fact, the bound is $O(1)$ ), we obtain

$$
\int_{-\infty}^{\infty}\left|\int_{-1}^{1} \int_{0}^{\infty} \cos (u \lambda) \omega(\lambda) \partial_{2} f_{2}(x, \lambda \sigma) d \lambda d \sigma\right| d u \leq C\langle x\rangle .
$$

Combining this bound with (8.16), (8.17), and (8.11), we conclude that

$$
\left\|\left[\omega(\lambda) F_{1}(x, \lambda) D^{-1}(\lambda) \underline{e}\right]^{\vee}\right\|_{1} \leq C\langle x\rangle \quad \forall x \in \mathbb{R},
$$

which in turn implies that

$$
\begin{aligned}
& \left|\int_{0}^{\infty} e^{i t \lambda^{2}} \omega(\lambda)\left\langle\phi, \sigma_{3} F_{1}(\cdot, \lambda) D^{-1}(\lambda) \underline{e}\right\rangle\left\langle F_{1}(\cdot, \lambda) D^{-1}(\lambda) \underline{e}, \psi\right\rangle d \lambda\right| \\
& \leq C|t|^{-\frac{1}{2}}\|\langle x\rangle \phi\|_{1}\|\langle y\rangle \psi\|_{1} .
\end{aligned}
$$


This is the desired estimate on (8.1), but only for the case when $\partial_{\lambda}$ falls on the factors not involving $F_{1}(\cdot, \lambda) D^{-1}(\lambda) \underline{e}$.

We now consider the case when $\partial_{\lambda}$ falls on $F_{1}(x, \lambda) D^{-1}(\lambda) \underline{e}$. Hence, we need to estimate

$$
\begin{aligned}
& \int_{0}^{\infty} e^{i t \lambda^{2}} \chi\left(\lambda^{2}\right) \lambda\left\langle\phi, \sigma_{3} \partial_{\lambda}\left[F_{1}(\cdot, \lambda) D^{-1}(\lambda)\right] \underline{e}\right\rangle\left\langle F_{1}(\cdot, \lambda) D^{-1}(\lambda) \underline{e}, \psi\right\rangle d \lambda \\
& =\int_{0}^{\infty} e^{i t \lambda^{2}} \chi\left(\lambda^{2}\right)\left\langle\phi, \sigma_{3} \partial_{\lambda}\left[\lambda F_{1}(\cdot, \lambda) D^{-1}(\lambda)\right] \underline{e}\right\rangle\left\langle F_{1}(\cdot, \lambda) D^{-1}(\lambda) \underline{e}, \psi\right\rangle d \lambda \\
& -\int_{0}^{\infty} e^{i t \lambda^{2}} \chi\left(\lambda^{2}\right)\left\langle\phi, \sigma_{3} F_{1}(\cdot, \lambda) D^{-1}(\lambda) \underline{e}\right\rangle\left\langle F_{1}(\cdot, \lambda) D^{-1}(\lambda) \underline{e}, \psi\right\rangle d \lambda .
\end{aligned}
$$

We have just estimated the final integral. Hence 8.23) is the main issue. By the same reductions as before, it will satisfy the desired bounds provided

$$
\left\|\left[\omega_{1}(\lambda) \partial_{\lambda}\left[\lambda F_{1}(x, \lambda) D^{-1}(\lambda) \underline{e}\right]\right]^{\wedge}\right\|_{1} \leq C\langle x\rangle
$$

where $\omega_{1}$ is a smooth cut-off. Now

$$
\begin{aligned}
& {\left[\omega_{1}(\lambda) \partial_{\lambda}\left[\lambda F_{1}(x, \lambda) D^{-1}(\lambda) \underline{e}\right]\right]^{\wedge}(u)} \\
& =i x \int_{0}^{\infty} \cos (u \lambda) e^{i x \lambda} \omega_{1}(\lambda) \lambda e^{-i x \lambda} f_{1}(x, \lambda)\left\langle\underline{e}, D^{-1}(\lambda) \underline{e}\right\rangle d \lambda \\
& \quad+\int_{0}^{\infty} \cos (u \lambda) e^{i x \lambda} \omega_{1}(\lambda) \partial_{\lambda}\left[\lambda e^{-i x \lambda} f_{1}(x, \lambda)\left\langle\underline{e}, D^{-1}(\lambda) \underline{e}\right\rangle\right] d \lambda \\
& \quad-\int_{0}^{\infty} \cos (u \lambda) x \mu^{\prime}(\lambda) \omega_{1}(\lambda) e^{-x \mu} \lambda e^{x \mu} f_{3}(x, \lambda)\left\langle\underline{e}^{\prime}, D^{-1}(\lambda) \underline{e}\right\rangle d \lambda \\
& \quad+\int_{0}^{\infty} \cos (u \lambda) \omega_{1}(\lambda) e^{-x \mu} \partial_{\lambda}\left[\lambda e^{x \mu} f_{3}(x, \lambda)\left\langle\underline{e}^{\prime}, D^{-1}(\lambda) \underline{e}\right\rangle\right] d \lambda .
\end{aligned}
$$

We again need to distinguish $x \geq 0$ from $x \leq 0$. We start with the former case. Integrating by parts in (8.24) leads to

$$
\begin{aligned}
& i x \int_{0}^{\infty} \cos (u \lambda) e^{i x \lambda} \omega_{1}(\lambda) \lambda e^{-i x \lambda} f_{1}(x, \lambda)\left\langle\underline{e}, D^{-1}(\lambda) \underline{e}\right\rangle d \lambda \\
& =-\frac{i x}{2 i(x+u)} \int_{0}^{\infty} e^{i(x+u) \lambda} \partial_{\lambda}\left[\omega_{1}(\lambda) \lambda e^{-i x \lambda} f_{1}(x, \lambda)\left\langle\underline{e}, D^{-1}(\lambda) \underline{e}\right\rangle\right] d \lambda \\
& \quad-\frac{i x}{2 i(x-u)} \int_{0}^{\infty} e^{i(x-u) \lambda} \partial_{\lambda}\left[\omega_{1}(\lambda) \lambda e^{-i x \lambda} f_{1}(x, \lambda)\left\langle\underline{e}, D^{-1}(\lambda) \underline{e}\right\rangle\right] d \lambda .
\end{aligned}
$$

It is important that boundary terms do not appear here (due to the $\lambda$ ). On the other hand, boundary terms do arise upon integrating (8.25) by parts. More precisely, 
for the case of (8.25) we obtain

$$
\begin{aligned}
& \int_{0}^{\infty} \cos (u \lambda) e^{i x \lambda} \omega_{1}(\lambda) \partial_{\lambda}\left[\lambda e^{-i x \lambda} f_{1}(x, \lambda)\left\langle\underline{e}, D^{-1}(\lambda) \underline{e}\right\rangle\right] d \lambda \\
& =-\left.\frac{1}{2 i(x+u)} \omega_{1}(0) \partial_{\lambda}\left[\lambda e^{-i x \lambda} f_{1}(x, \lambda)\left\langle\underline{e}, D^{-1}(\lambda) \underline{e}\right\rangle\right]\right|_{\lambda=0} \\
& -\left.\frac{1}{2 i(x-u)} \omega_{1}(0) \partial_{\lambda}\left[\lambda e^{-i x \lambda} f_{1}(x, \lambda)\left\langle\underline{e}, D^{-1}(\lambda) \underline{e}\right\rangle\right]\right|_{\lambda=0} \\
& \quad-\frac{1}{2 i(x+u)} \int_{0}^{\infty} e^{i(x+u) \lambda} \partial_{\lambda}\left[\omega_{1}(\lambda) \partial_{\lambda}\left[\lambda e^{-i x \lambda} f_{1}(x, \lambda)\left\langle\underline{e}, D^{-1}(\lambda) \underline{e}\right\rangle\right]\right] d \lambda \\
& \quad-\frac{1}{2 i(x-u)} \int_{0}^{\infty} e^{i(x-u) \lambda} \partial_{\lambda}\left[\omega_{1}(\lambda) \partial_{\lambda}\left[\lambda e^{-i x \lambda} f_{1}(x, \lambda)\left\langle\underline{e}, D^{-1}(\lambda) \underline{e}\right\rangle\right]\right] d \lambda
\end{aligned}
$$

Integrating by parts one more time in (8.28) and (8.29) implies

$$
\begin{aligned}
& \left|i x \int_{0}^{\infty} \cos (u \lambda) e^{i x \lambda} \omega_{1}(\lambda) \lambda e^{-i x \lambda} f_{1}(x, \lambda) d \lambda\right| \\
& \leq C|x|(1+|x-u|)^{-2}+C|x|(1+|x+u|)^{-2}
\end{aligned}
$$

uniformly in $x \geq 0$, whereas 88.30 -8.33) are treated the same way as 8.8). Consequently, (8.24) and (8.25) each have $L^{1}(d u)$ norm $\leq C\langle x\rangle$ provided $x \geq 0$. Integrating (8.26) and (8.27) in $\lambda$ twice yields

$$
|\underline{(8.26)}|+|\underline{(8.27)}| \leq C\left(1+u^{2}\right)^{-1}
$$

uniformly in $x \geq 0$ since for $\ell=0,1,2$

$$
\begin{aligned}
& \sup _{x \geq 0}\left|\partial_{\lambda}^{\ell}\left[x \mu^{\prime}(\lambda) \omega_{1}(\lambda) e^{-x \mu} \lambda e^{x \mu} f_{3}(x, \lambda)\left\langle\underline{e}^{\prime}, D^{-1}(\lambda) \underline{e}\right\rangle\right]\right| \leq C, \\
& \sup _{x \geq 0}\left|\partial_{\lambda}^{\ell}\left[x \mu^{\prime}(\lambda) \omega_{1}(\lambda) e^{-x \mu} \lambda e^{x \mu} f_{3}(x, \lambda)\left\langle\underline{e}^{\prime}, D^{-1}(\lambda) \underline{e}\right\rangle\right]\right| \leq C .
\end{aligned}
$$

Hence, (8.26) and (8.27) each have $L^{1}(d u)$ norm $\leq C$ provided $x \geq 0$.

To deal with $x \leq 0$, we use (8.12):

$$
2 i \lambda F_{1}(x, \lambda) D^{-1}(\lambda) \underline{e}=f_{2}(-x, \lambda)+r(\lambda) f_{1}(-x, \lambda)+f_{3}(-x, \lambda)\left\langle 2 i \lambda q B(\lambda) D^{-1}(\lambda) \underline{e}, \underline{e}^{\prime}\right\rangle .
$$

This implies that

$$
\begin{aligned}
& 2 i\left[\omega_{1}(\lambda) \partial_{\lambda}\left[\lambda F_{1}(x, \lambda) D^{-1}(\lambda) \underline{e}\right]\right]^{\wedge}(u) \\
& =\int_{0}^{\infty} \cos (\lambda u) \omega_{1}(\lambda) \partial_{\lambda} f_{2}(-x, \lambda) d \lambda+\int_{0}^{\infty} \cos (\lambda u) \omega_{1}(\lambda) \partial_{\lambda}\left[r(\lambda) f_{1}(-x, \lambda)\right] d \lambda \\
& \quad+\int_{0}^{\infty} \cos (\lambda u) \omega_{1}(\lambda) \partial_{\lambda}\left[f_{3}(-x, \lambda)\left\langle 2 i \lambda q B(\lambda) D^{-1}(\lambda) \underline{e}, \underline{e}^{\prime}\right\rangle\right] d \lambda,
\end{aligned}
$$


which further simplifies to

$$
\begin{aligned}
= & \int_{0}^{\infty} \cos (\lambda u) e^{i x \lambda} \omega_{1}(\lambda) \partial_{\lambda}\left[e^{-i x \lambda} f_{2}(-x, \lambda)\right] d \lambda \\
+ & \int_{0}^{\infty} \cos (\lambda u) e^{-i x \lambda} \omega_{1}(\lambda) \partial_{\lambda}\left[r(\lambda) e^{i x \lambda} f_{1}(-x, \lambda)\right] d \lambda \\
& +i x \int_{0}^{\infty} \cos (\lambda u) e^{i x \lambda} \omega_{1}(\lambda) e^{i x \lambda} f_{2}(-x, \lambda) d \lambda \\
- & i x \int_{0}^{\infty} \cos (\lambda u) e^{-i x \lambda} \omega_{1}(\lambda) r(\lambda) e^{i x \lambda} f_{1}(-x, \lambda) d \lambda \\
& +\int_{0}^{\infty} \cos (\lambda u) x \mu^{\prime}(\lambda) e^{x \mu} \omega_{1}(\lambda) e^{-x \mu} f_{3}(-x, \lambda)\left\langle 2 i \lambda q B(\lambda) D^{-1}(\lambda) \underline{e}, \underline{e}^{\prime}\right\rangle d \lambda \\
& +\int_{0}^{\infty} \cos (\lambda u) e^{x \mu} \omega_{1}(\lambda) \partial_{\lambda}\left[e^{-x \mu} f_{3}(-x, \lambda)\left\langle 2 i \lambda q B(\lambda) D^{-1}(\lambda) \underline{e}, \underline{e}^{\prime}\right\rangle\right] d \lambda .
\end{aligned}
$$

The two integrals in (8.34) (which are not preceded by $i x$ ) are integrated by parts in the same way as (8.30) most $C\langle x\rangle$ with a constant uniform in $x \leq 0$. The integrals in (8.35), which are preceded by $i x$ are also integrated by parts, but we need to check in this case that the boundary terms $\lambda=0$ cancel each other. However, these boundary terms are

$$
\begin{aligned}
& \frac{x}{2(u-x)} \omega_{1}(0) r(0) f_{1}(-x, 0)-\frac{x}{2(u+x)} \omega_{1}(0) r(0) f_{1}(-x, 0) \\
& -\frac{x}{2(u+x)} \omega_{1}(0) f_{2}(-x, 0)-\frac{x}{2(x-u)} \omega_{1}(0) f_{2}(-x, 0)=0,
\end{aligned}
$$

since $r(0)=-1$ and $f_{1}(-x, 0)=f_{2}(-x, 0)$. Hence, (8.35) can be treated as (8.28), (8.29). Finally, (8.36) and (8.37) are $\leq C\left(1+u^{2}\right)^{-1}$ uniformly in $x \leq 0$ (see (8.26), (8.27)), and we are done.

Interpolating this estimate with the unweighted $L^{1}(\mathbb{R}) \rightarrow L^{\infty}(\mathbb{R})$ as well as the $L^{2}$ bound yields the following:

Lemma 8.2. Let $\mathcal{H}$ be admissible; see Definition 6.1. Then for all $0 \leq \theta \leq 1$, all $1 \leq p \leq 2$, and all $t \neq 0$,

$$
\left\|\langle x\rangle^{-\theta\left(\frac{1}{p}-\frac{1}{p^{\prime}}\right)} e^{i t \mathcal{H}} P_{s} f\right\|_{p^{\prime}} \leq C|t|^{-\left(\frac{1}{2}+\theta\right)\left(\frac{1}{p}-\frac{1}{p^{\prime}}\right)}\left\|\langle x\rangle^{\theta\left(\frac{1}{p}-\frac{1}{p^{\prime}}\right)} f\right\|_{p} .
$$

Here $C$ is some absolute constant.

Proof. Interpolating between Propositions 7.1 and 8.1 yields

$$
\left\|\langle x\rangle^{-\theta} e^{i t \mathcal{H}} P_{s} f\right\|_{\infty} \leq C|t|^{-\frac{1}{2}-\theta}\left\|\langle x\rangle^{\theta} f\right\|_{1} .
$$

The lemma now follows from a further interpolation with Lemma 6.11

Just as in the unweighted case, derivatives can be introduced here as well. We restrict ourselves to two derivatives, although more are possible.

Corollary 8.3. Let $\mathcal{H}$ be admissible; see Definition 6.1. Then for all $0 \leq \theta \leq 1$, all $1<p \leq 2$, and all $t \neq 0$,

$$
\left\|\langle x\rangle^{-\theta\left(\frac{1}{p}-\frac{1}{p^{\prime}}\right)} e^{i t \mathcal{H}} P_{s} f\right\|_{W^{k, p^{\prime}}(\mathbb{R})} \leq C|t|^{-\left(\frac{1}{2}+\theta\right)\left(\frac{1}{p}-\frac{1}{p^{\prime}}\right)} \sum_{j=0}^{k}\left\|\langle x\rangle^{\theta\left(\frac{1}{p}-\frac{1}{p^{\prime}}\right)} \partial_{x}^{j} f\right\|_{L^{p}(\mathbb{R})}
$$


for all $0 \leq k \leq 2$. Alternatively,

$$
\left\|\langle x\rangle^{-\theta\left(\frac{1}{p}-\frac{1}{p^{\prime}}\right)} \partial_{x}^{k} e^{i t \mathcal{H}} P_{s} f\right\|_{L^{p^{\prime}}(\mathbb{R})} \leq C|t|^{-\left(\frac{1}{2}+\theta\right)\left(\frac{1}{p}-\frac{1}{p^{\prime}}\right)} \sum_{j=0}^{k}\left\|\langle x\rangle^{\theta\left(\frac{1}{p}-\frac{1}{p^{\prime}}\right)} \partial_{x}^{j} f\right\|_{L^{p}(\mathbb{R})}
$$

for all $0 \leq k \leq 2$. Here $C$ is a constant that depends on $p$.

Proof. The case $k=0$ is Lemma 8.2 Let $a>0$ be large. As in the proof of Corollary 7.2, for $p^{\prime}<\infty$,

$$
\begin{aligned}
& \left\|\langle x\rangle^{-\theta\left(\frac{1}{p}-\frac{1}{p^{\prime}}\right)} e^{i t \mathcal{H}} P_{s} f\right\|_{W^{2, p^{\prime}}(\mathbb{R})} \\
& \leq C\left\|(\mathcal{H}-i a)\langle x\rangle^{-\theta\left(\frac{1}{p}-\frac{1}{p^{\prime}}\right)} e^{i t \mathcal{H}} P_{s} f\right\|_{p^{\prime}} \\
& \leq C\left\|\langle x\rangle^{-\theta\left(\frac{1}{p}-\frac{1}{p^{\prime}}\right)} e^{i t \mathcal{H}} P_{s}(\mathcal{H}-i a) f\right\|_{p^{\prime}}+\left\|\left[\langle x\rangle^{-\theta\left(\frac{1}{p}-\frac{1}{p^{\prime}}\right)}, \mathcal{H}\right] e^{i t \mathcal{H}} P_{s} f\right\|_{p^{\prime}} \\
& \leq C|t|^{-\left(\frac{1}{2}+\theta\right)\left(\frac{1}{p}-\frac{1}{p^{\prime}}\right)}\left\|\langle x\rangle^{\theta\left(\frac{1}{p}-\frac{1}{p^{\prime}}\right)}(\mathcal{H}-i a) f\right\|_{p}+\left\|\left[\langle x\rangle^{-\theta\left(\frac{1}{p}-\frac{1}{p^{\prime}}\right)}, \mathcal{H}\right] e^{i t \mathcal{H}} P_{s} f\right\|_{p^{\prime}} .
\end{aligned}
$$

The first term on the right can be controlled in terms of ordinary derivatives, whereas the second only involves derivatives of order zero and one. Therefore, this second term can be controlled by means of interpolation. Hence, (8.38) holds. The second inequality follows from the first by induction in $k$.

\section{The SPECTRum of the LINEARIZED NLS EQUATION}

The linearization of the NLS equation

$$
i \partial_{t} \psi+\partial_{x x} \psi=-|\psi|^{2 \sigma} \psi
$$

around the ground state

$$
\phi(x)=(\sigma+1)^{\frac{1}{2 \sigma}} \cosh ^{-\frac{1}{\sigma}}(\sigma x)
$$

of $-\partial_{x x} \phi+\phi-\phi^{2 \sigma+1}=0$ leads to the operator

$$
\mathcal{H}=\left(\begin{array}{cc}
0 & i L_{-} \\
-i L_{+} & 0
\end{array}\right)
$$

where

$$
\begin{aligned}
& L_{-}=-\partial_{x x}+1-\phi^{2 \sigma}=-\partial_{x x}+1-(\sigma+1) \cosh ^{-2}(\sigma x), \\
& L_{+}=-\partial_{x x}+1-(2 \sigma+1) \phi^{2 \sigma}=-\partial_{x x}+1-(2 \sigma+1)(\sigma+1) \cosh ^{-2}(\sigma x) .
\end{aligned}
$$

Equivalently, $\mathcal{H}$ can be written in the form

$$
\mathcal{H}=\left(\begin{array}{cc}
-\partial_{x x}+1-(\sigma+1) \phi^{2 \sigma} & -\sigma \phi^{2 \sigma} \\
\sigma \phi^{2 \sigma} & \partial_{x x}-1+(\sigma+1) \phi^{2 \sigma}
\end{array}\right) .
$$

Lemma 9.1. If $\sigma>1$, then $L_{+}$and $L_{-}$have the following properties: they have no eigenvalues in the interval $(0,1]$ and for both $L_{+}$and $L_{-}$the threshold 1 is not a resonance.

Proof. A "resonance" of $L_{\mp}$ at energy one means one of the following equivalent things:

- There is a solution $f \in L^{\infty} \backslash L^{2}$ of $L_{\mp} f=f$.

- The two Jost solutions with energy one are linearly dependent.

- The transmission coefficient at energy one does not vanish. 
The lemma can easily be deduced from Flügge [15; for example, see Problem 39 on page 94 of [15]. It is shown there that the Hamiltonian $H=-\frac{d^{2}}{d x^{2}}-\alpha^{2} \frac{\lambda(\lambda-1)}{\cosh ^{2}(\alpha x)}$ with $\lambda>1$ has a zero-energy resonance (which is the same as $T(0)=0$ for the transmission coefficient $T(E)$ ) iff $\lambda$ is an integer. In the case of $L_{-}, \alpha=\sigma$ and $\lambda=\frac{1}{2}+\frac{1}{2} \sqrt{1+4 \frac{\sigma+1}{\sigma^{2}}}$. If $\lambda=n \geq 2$, then $\frac{\sigma+1}{\sigma^{2}}=n(n-1) \geq 2$, which implies that $\sigma \leq 1$. Moreover, it is shown there that the number of negative bound states of $H$ is the largest integer $\leq \lambda$. Here $\lambda<2$ iff $\sigma>1$ so that there is exactly one bound state of $L_{-}$below energy one iff $\sigma>1$. For $L_{+}$, we have

$$
\lambda=\frac{1}{2}+\frac{1}{2} \sqrt{1+4 \frac{(2 \sigma+1)(\sigma+1)}{\sigma^{2}}}<3
$$

iff $\sigma>1$. This implies that $L_{+}$has exactly two bound states below energy one, as desired. In the same way, it can be checked that $L_{+}$does not have a resonance at 1 if $\sigma>1$.

The main result of this section is the following proposition.

Proposition 9.2. For any $\sigma \geq 2$ the operator $\mathcal{H}$ on $L^{2}(\mathbb{R}) \times L^{2}(\mathbb{R})$ with domain $W^{2,2}(\mathbb{R}) \times W^{2,2}(\mathbb{R})$ satisfies:

- The spectrum of $\mathcal{H}$, denoted by $\operatorname{spec}(\mathcal{H})$, is contained in $\mathbb{R} \cup i \mathbb{R}$.

- The essential spectrum equals $(-\infty,-1] \cup[1, \infty)$. There are no embedded eigenvalues in the essential spectrum.

- The only real eigenvalue in $[-1,1]$ is zero. The geometric multiplicity of the zero eigenvalue is two, and its algebraic multiplicity equals four or six, depending on whether $\sigma>2$ or $\sigma=2$.

- If $\sigma>2$, then there is a unique pair of imaginary eigenvalues $\pm i \gamma, \gamma>0$, which are both simple.

- The edges \pm 1 are not resonance 23 .

In particular, $\mathcal{H}$ is admissible for all $\sigma \geq 2$.

Proof. We will rely on the techniques from [5] and [36]. The latter paper only deals with the critical case $\sigma=2$, which means that we need to adapt some of Perelman's arguments to the supercritical case $\sigma>2$.

The statement about the essential spectrum follows from Weyl's criterion via the symmetric resolvent identity; see [21. It will be convenient to introduce the ground state $\phi(x, \alpha)$ (which also depends on $\sigma$ ) of

$$
-\partial_{x x} \phi+\alpha^{2} \phi-\phi^{2 \sigma+1}=0 .
$$

Then $\phi(x, \alpha)=\alpha^{\frac{1}{\sigma}} \phi(\alpha x, 1)$. Similarly,

$$
\begin{aligned}
& L_{-}=-\partial_{x x}+\alpha^{2}-\phi^{2 \sigma}, \\
& L_{+}=-\partial_{x x}+\alpha^{2}-(2 \sigma+1) \phi^{2 \sigma} .
\end{aligned}
$$

Clearly, $L_{-} \phi=0, L_{+}\left(\partial_{\alpha} \phi\right)=-2 \alpha \phi$, and $L_{+}\left(\phi^{\prime}\right)=0$. Since $\phi>0$, it follows that $L_{-} \geq 0$. Also, $\operatorname{ker}\left(L_{+}\right)=\operatorname{span}\left\{\phi^{\prime}\right\}$ (by the simplicity of eigenvalues in the one-dimensional case), and $L_{+}$has a unique negative eigenvalue $E_{0}<0$. If $\mathcal{H} f=z f$ with $f=\left(\begin{array}{l}f_{1} \\ f_{2}\end{array}\right) \in L^{2} \backslash\{0\}$ and $z \neq 0$, then $L_{-} L_{+} f_{1}=z^{2} f_{1}$ and thus

\footnotetext{
${ }^{23}$ See Definition 5.18

${ }^{24}$ See Definition 6.1
} 
$\sqrt{L_{-}} L_{+} \sqrt{L_{-}} g_{1}=z^{2} g_{1}$ where $g_{1}=L_{-}^{-\frac{1}{2}} f_{1}$ (note that $f_{1} \perp \phi$ ). It follows that $z^{2} \in \mathbb{R}$. Moreover, this argument can be refined (see page 1137 in [5]) to show that if $z \neq 0$, then the geometric and algebraic multiplicities of the eigenvalue $z$ are equal. If $\mathcal{H}$ has imaginary eigenvalues, then some $z^{2}<0$ would need to be an eigenvalue of $\sqrt{L_{-}} L_{+} \sqrt{L_{-}}$. This implies that

$$
\lambda_{0}:=\min _{\|f\|_{2}=1, f \perp \phi}\left\langle L_{+} \phi, \phi\right\rangle<0 .
$$

If $g$ is a minimizer here, then by Lagrange multipliers

$$
\left(L_{+}-\lambda_{0}\right) g=c \phi, \quad 0=\langle g, \phi\rangle=c\left\langle\left(L_{+}-\lambda_{0}\right)^{-1} \phi, \phi\right\rangle .
$$

Since $c \neq 0$ (otherwise, $g>0$ is the ground state of $L_{+}$which contradicts $g \perp \phi$ ), one has $h\left(\lambda_{0}\right)=0$ where

$$
h(\lambda):=\left\langle\left(L_{+}-\lambda\right)^{-1} \phi, \phi\right\rangle .
$$

But $h(\lambda)$ is strictly increasing which requires

$$
0<h(0)=\left\langle L_{+}^{-1} \phi, \phi\right\rangle=-\frac{1}{2 \alpha}\left\langle\partial_{\alpha} \phi, \phi\right\rangle .
$$

Since

$$
\partial_{\alpha}\|\phi\|_{2}^{2}=2\left\langle\partial_{\alpha} \phi, \phi\right\rangle<0 \text { iff } \sigma>2,
$$

it follows that $\operatorname{spec}(\mathcal{H}) \subset \mathbb{R}$ if $\sigma \leq 2$ (we will see later that this is if and only if).

As far as the zero eigenvalue is concerned, note that (use (9.1))

$$
\mathcal{H}\left(\begin{array}{l}
0 \\
\phi
\end{array}\right)=0, \mathcal{H}\left(\begin{array}{c}
\phi^{\prime} \\
0
\end{array}\right)=0, \mathcal{H}\left(\begin{array}{c}
0 \\
x \phi
\end{array}\right)=\left(\begin{array}{c}
-2 i \phi^{\prime} \\
0
\end{array}\right), \mathcal{H}\left(\begin{array}{c}
\partial_{\alpha} \phi \\
0
\end{array}\right)=\left(\begin{array}{c}
0 \\
2 i \alpha \phi
\end{array}\right) .
$$

It is clear that this describes $\operatorname{ker}(\mathcal{H})$ and $\operatorname{ker}\left(\mathcal{H}^{2}\right)$ completely. Moreover, it is easy to check that $\operatorname{ker}\left(\mathcal{H}^{k}\right)=\operatorname{ker}\left(\mathcal{H}^{2}\right)$ if $k \geq 3$ provided $\sigma \neq 2$. Indeed, it is enough to check this for $k=3$ and that case is settled by writing out the third power of (9.1) explicitly; see [55], page 485. In the critical case $\sigma=2$, one has $\partial_{\alpha} \phi \perp \phi$. Hence $\partial_{\alpha} \phi \in \operatorname{Ran}\left(L_{-}\right)$. In fact, direct differentiation shows that $L_{-}\left(x^{2} \phi\right)=-4 \alpha \partial_{\alpha} \phi$. Finally, since $x^{2} \phi \perp \phi^{\prime}$, it follows that $L_{+} \rho=x^{2} \phi$ for some $\rho$. In summary,

$$
\mathcal{H}\left(\begin{array}{c}
0 \\
x^{2} \phi
\end{array}\right)=\left(\begin{array}{c}
-4 i \alpha \partial_{\alpha} \phi \\
0
\end{array}\right), \quad \mathcal{H}\left(\begin{array}{l}
\rho \\
0
\end{array}\right)=\left(\begin{array}{c}
0 \\
-i x^{2} \phi
\end{array}\right)
$$

for the case $\sigma=2$. This shows that $\operatorname{ker}\left(\mathcal{H}^{2}\right) \subsetneq \operatorname{ker}\left(\mathcal{H}^{3}\right) \subsetneq \operatorname{ker}\left(\mathcal{H}^{4}\right)$ and the codimensions in each case equal one. Again, it is easy to check that in this case $\operatorname{ker}\left(\mathcal{H}^{k}\right)=\operatorname{ker}\left(\mathcal{H}^{4}\right)$ for $k \geq 4$.

Let us now assume that the only real eigenvalue of $\mathcal{H}$ in $[-1,1]$ is zero for all $\sigma \geq 2$ (this is false if $\sigma<2$ ). Then by the continuous dependence of the Riesz projection onto the discrete spectrum of $\mathcal{H}$ on the power $\sigma$, it follows that the rank of this projection is always equal to six. Hence, if $\sigma>2$, then there are two imaginary eigenvalues counted with multiplicity. By the commutation properties relative to the Pauli matrices (5.2), it follows that this has to be a pair $\pm i \gamma, \gamma>0$, of simple eigenvalues.

It remains to show the following three properties:

(i) The only real eigenvalue in $[-1,1]$ is zero.

(ii) The edges \pm 1 are not resonances.

(iii) There are no embedded eigenvalues in the essential spectrum. 
Perelman proved these statements for $\sigma=2$; cf. Sections 2.1.2 and 2.1.3 of [36]. Hence, it will suffice to consider the case $\sigma>2$ which can be dealt with by adapting Perelman's arguments. We will rely on Lemma 9.1 for that purpose.

Suppose (i) fails. Then $\mathcal{H}(\alpha)^{2}$ has an eigenvalue $E \in\left(0, \alpha^{4}\right]$. For simplicity and without loss of generality, let us choose $\alpha=1$. Then there is $\psi \in L^{2}(\mathbb{R}), \psi \neq 0$, such that

$$
L_{-} L_{+} \psi=E \psi
$$

with $0<E \leq 1$. Clearly, $\psi \perp \phi$ and $\psi \in H_{l o c}^{4}(\mathbb{R})$ by elliptic regularity. Define $A:=P L_{+} P$ where $P$ is the projection orthogonal to $\phi$. Since $\left\langle\phi, \partial_{\alpha} \phi\right\rangle \neq 0$, we conclude that

$$
\operatorname{ker}(A)=\operatorname{span}\left\{\phi^{\prime}, \phi\right\} .
$$

Moreover, let $E_{0}<0$ be the unique negative eigenvalue of $L_{+}$. Then consider (as before) the function

$$
h(\lambda):=\left\langle\left(L_{+}-\lambda\right)^{-1} \phi, \phi\right\rangle
$$

which is differentiable on the interval $\left(E_{0}, 1\right)$ due to the orthogonality of $\phi$ to the kernel of $L_{+}$. Moreover,

$$
h^{\prime}(\lambda)=\left\langle\left(L_{+}-\lambda\right)^{-2} \phi, \phi\right\rangle>0, h(0)=-\frac{1}{2}\left\langle\phi, \partial_{\alpha} \phi\right\rangle>0 .
$$

The final inequality here is due to the supercritical nature of our problem. Since also $h(\lambda) \rightarrow-\infty$ as $\lambda \rightarrow E_{0}$, it follows that $h\left(\lambda_{1}\right)=0$ for some $E_{0}<\lambda_{1}<0$. Moreover, this is the only zero of $h(\lambda)$ with $E_{0}<\lambda<1$. If we set

$$
\tilde{\eta}:=\left(L_{+}-\lambda_{1}\right)^{-1} \phi
$$

then

Conversely, if

$$
A \tilde{\eta}=\lambda_{1} \tilde{\eta}, \quad\langle\tilde{\eta}, \phi\rangle=0 .
$$

$$
A f=\lambda f
$$

for some $E_{0}<\lambda<1, \lambda \neq 0$, and $f \in L^{2}(\mathbb{R})$, then $f \perp \phi$ and

$$
\left(P L_{+} P-\lambda\right) f=(A-\lambda) f=0 .
$$

Since also

$$
E_{0}\langle f, f\rangle \leq\left\langle L_{+} f, f\right\rangle=\lambda\langle f, f\rangle,
$$

it follows that $\lambda \geq E_{0}$. If $\lambda=E_{0}$, then $f$ would necessarily have to be the ground state of $L_{+}$and thus of definite sign. But then $\langle f, \phi\rangle \neq 0$, which is impossible. Hence $E_{0}<\lambda<1$. But then $h(\lambda)=0$ implies that $\lambda=\lambda_{1}$ is unique. In summary, $A$ has eigenvalues $\lambda_{1}$ and 0 in $(-\infty, 1)$, with $\lambda_{1}$ being a simple eigenvalue and 0 being of multiplicity two. Now define

$$
\mathcal{F}:=\operatorname{span}\left\{\psi, \tilde{\eta}, \phi^{\prime}, \phi\right\} .
$$

We claim that

$$
\operatorname{dim}(\mathcal{F})=4 .
$$

Since $\phi$ is perpendicular to the other functions, it suffices to show that

$$
c_{1} \psi+c_{2} \tilde{\eta}+c_{3} \phi^{\prime}=0
$$

can only be the trivial linear combination. Apply $L_{+}$. Then

$$
c_{1} L_{+} \psi+c_{2} L_{+} \tilde{\eta}=0
$$


and therefore

$$
\begin{gathered}
c_{1}\left\langle L_{+} \psi, \psi\right\rangle+c_{2}\left\langle L_{+} \tilde{\eta}, \psi\right\rangle=0, \\
c_{1}\left\langle L_{+} \psi, \tilde{\eta}\right\rangle+c_{2}\left\langle L_{+} \tilde{\eta}, \tilde{\eta}\right\rangle=0 .
\end{gathered}
$$

This is the same as

$$
\begin{aligned}
c_{1} E\left\langle L_{-}^{-1} \psi, \psi\right\rangle+c_{2} \lambda_{1}\langle\tilde{\eta}, \psi\rangle & =0, \\
c_{1} \lambda_{1}\langle\psi, \tilde{\eta}\rangle+c_{2} \lambda_{1}\langle\tilde{\eta}, \tilde{\eta}\rangle & =0 .
\end{aligned}
$$

The determinant of this system is

$$
E \lambda_{1}\left\langle L_{-}^{-1} \psi, \psi\right\rangle\langle\tilde{\eta}, \tilde{\eta}\rangle-\lambda_{1}^{2}|\langle\tilde{\eta}, \psi\rangle|^{2}<0
$$

Hence $c_{1}=c_{2}=0$ and therefore also $c_{3}=0$, as desired. Thus, (9.3) holds. Finally, we claim that

$$
\sup _{\|f\|_{2}=1, f \in \mathcal{F}}\langle A f, f\rangle<1 .
$$

If this is true, then by the min-max principle and (9.3) we would obtain that the number of eigenvalues of $A$ in the interval $(-\infty, 1)$ (counted with multiplicity) would have to be at least four. On the other hand, we showed before that this number is exactly three, leading to a contradiction. Hence, we need to verify (9.4). Since $\left\langle P L_{-}^{-1} P f, f\right\rangle\langle\langle f, f\rangle$ for all $f \neq 0$ and since $E \leq 1$ by assumption, this in turn follows from the stronger claim that

$$
\langle A f, f\rangle \leq E\left\langle P L_{-}^{-1} P f, f\right\rangle
$$

for all $f=a \psi+b \phi+c \phi^{\prime}+d \tilde{\eta}$. Clearly, we can take $b=0$. Then the left-hand side of $(9.5)$ is equal to

$$
\begin{aligned}
& \left\langle L_{+}(a \psi), a \psi+c \phi^{\prime}+d \tilde{\eta}\right\rangle+\left\langle L_{+}\left(c \phi^{\prime}+d \tilde{\eta}\right), a \psi+c \phi^{\prime}+d \tilde{\eta}\right\rangle \\
& =E\left\langle L_{-}^{-1}(a \psi), a \psi+c \phi^{\prime}+d \tilde{\eta}\right\rangle+E\left\langle c \phi^{\prime}+d \tilde{\eta}, L_{-}^{-1}(a \psi)\right\rangle+\left\langle L_{+}(d \tilde{\eta}), d \tilde{\eta}\right\rangle \\
& =E\left\langle L_{-}^{-1}(a \psi), a \psi+c \phi^{\prime}+d \tilde{\eta}\right\rangle+E\left\langle c \phi^{\prime}+d \tilde{\eta}, L_{-}^{-1}(a \psi)\right\rangle+\lambda_{1}\|d \tilde{\eta}\|_{2}^{2},
\end{aligned}
$$

whereas the right-hand side of (9.5) is

$=E\left\langle L_{-}^{-1}(a \psi), a \psi+c \phi^{\prime}+d \tilde{\eta}\right\rangle+E\left\langle c \phi^{\prime}+d \tilde{\eta}, L_{-}^{-1}(a \psi)\right\rangle+E\left\langle L_{-}^{-1}\left(c \phi^{\prime}+d \tilde{\eta}\right), c \phi^{\prime}+d \tilde{\eta}\right\rangle$.

Since

$$
\lambda_{1}\|d \tilde{\eta}\|_{2}^{2} \leq 0, \quad E\left\langle L_{-}^{-1}\left(c \phi^{\prime}+d \tilde{\eta}\right), c \phi^{\prime}+d \tilde{\eta}\right\rangle \geq 0
$$

we see that (9.7) does indeed dominate (9.6), and (9.5) follows.

Next, we turn to the resonances. Suppose $\mathcal{H} f=f$ where $f \in L^{\infty} \backslash L^{2}(\mathbb{R})$. By Lemma 5.19, $f=C_{ \pm}\left(\begin{array}{l}0 \\ 1\end{array}\right)+O\left(e^{\mp \gamma x}\right)$ as $x \rightarrow \pm \infty$ where $C_{+} \neq 0$ and $C_{-} \neq 0$. Hence there exists $\psi \in L^{\infty} \backslash L^{2}$ (the first component of $f$ ) so that $\psi=C_{ \pm}+O\left(e^{\mp \gamma x}\right)$ as $x \rightarrow \pm \infty$ and such that $L_{-} L_{+} \psi=\psi$. This asymptotic expansion can also be differentiated. Pick a smooth cut-off $\chi \geq 0$ which is equal to one around zero and compactly supported. Define for any $0<\varepsilon<1$

$$
\psi^{\varepsilon}:=\psi \chi(\varepsilon \cdot)+\mu(\varepsilon) \phi, \quad \mu(\varepsilon):=-\frac{\langle\psi \chi(\varepsilon \cdot), \phi\rangle}{\langle\phi, \phi\rangle} .
$$

Clearly, $\left\langle\psi^{\varepsilon}, \phi\right\rangle=0$ and $|\mu(\varepsilon)|=o(1)$ as $\varepsilon \rightarrow 0$ (in fact, like $e^{-C / \varepsilon}$ ). It follows that

$$
\left\|\psi^{\varepsilon}\right\|_{2}^{2}=M_{0}(\varepsilon)+o(1), \quad M_{0}(\varepsilon):=\int_{\mathbb{R}}|\psi(x)|^{2} \chi(\varepsilon x)^{2} d x
$$


with $M_{0}(\varepsilon) \rightarrow \infty$ as $\varepsilon \rightarrow 0$. We now claim that

$$
\left\langle L_{+} \psi^{\varepsilon}, \psi^{\varepsilon}\right\rangle=\left\|\psi^{\varepsilon}\right\|_{2}^{2}+\left\langle\left(L_{+}-1\right) \psi, \psi\right\rangle+o(1)
$$

as $\varepsilon \rightarrow 0$. From the asymptotic behavior of $\psi$ it is clear that $M_{1}:=\left\langle\left(L_{+}-1\right) \psi, \psi\right\rangle$ is a finite expression. Write $L_{-}=-\partial_{x}^{2}+1+V_{1}$ and $L_{+}=-\partial_{x}^{2}+1+V_{2}$, with Schwartz functions $V_{1}, V_{2}$ (they are of course explicitly given in terms of $\phi$, but we are not going to use that now). We start from the evident expression

$$
\left\langle L_{+} \psi^{\varepsilon}, \psi^{\varepsilon}\right\rangle=\left\|\psi^{\varepsilon}\right\|_{2}^{2}+\left\langle\left(L_{+}-1\right) \psi^{\varepsilon}, \psi^{\varepsilon}\right\rangle=\left\|\psi^{\varepsilon}\right\|_{2}^{2}+\left\langle\left(-\partial_{x}^{2}+V_{2}\right) \psi^{\varepsilon}, \psi^{\varepsilon}\right\rangle .
$$

By the rapid decay of $V_{2}$,

$$
\left\langle\left(-\partial_{x}^{2}+V_{2}\right) \psi^{\varepsilon}, \psi^{\varepsilon}\right\rangle=\int_{\mathbb{R}}\left|\partial \psi^{\varepsilon}(x)\right|^{2} d x+\int_{\mathbb{R}} V_{2}(x)|\psi(x)|^{2} d x+o(1) .
$$

Since $\psi^{\prime} \in L^{2}$, we calculate further that

$$
\begin{aligned}
\int_{\mathbb{R}}\left|\partial \psi^{\varepsilon}(x)\right|^{2} d x= & \int_{\mathbb{R}}\left|\psi^{\prime}(x) \chi(\varepsilon x)+\varepsilon \psi(x) \chi^{\prime}(\varepsilon x)\right|^{2} d x \\
= & \int_{\mathbb{R}}\left|\psi^{\prime}(x)\right|^{2} d x+\int_{\mathbb{R}}\left|\psi^{\prime}(x)\right|^{2}\left(\chi(\varepsilon x)^{2}-1\right) d x \\
& +2 \varepsilon \int_{\mathbb{R}} \psi(x) \chi(\varepsilon x) \psi^{\prime}(x) \cdot \chi^{\prime}(\varepsilon x) d x+\varepsilon^{2} \int_{\mathbb{R}} \psi(x)^{2}\left|\chi^{\prime}(\varepsilon x)\right|^{2} d x \\
= & \int_{\mathbb{R}}\left|\psi^{\prime}(x)\right|^{2} d x+o(1) .
\end{aligned}
$$

To pass to the last line, use the asymptotics of $\psi$ and $\psi^{\prime}$. By these asymptotics, $f:=\left(L_{+}-1\right) \psi$ is rapidly decaying. Hence, $\left\langle\left(L_{+}-1\right) \psi, \psi\right\rangle=\langle f, \psi\rangle$ is well defined as a usual scalar product. Moreover, one has

$$
L_{-} f=-\left(L_{-}-1\right) \psi \text { or } \psi=-\left(L_{-}-1\right)^{-1} L_{-} f=-f-\left(L_{-}-1\right)^{-1} f .
$$

We conclude that

$$
\langle f+\psi, f\rangle=-\left\langle\left(L_{-}-1\right)^{-1} f, f\right\rangle<0,
$$

where the final inequality follows from $L_{-} \geq 1$ on $\{\phi\}^{\perp}$, as well as from the property that $L_{-}$has neither an eigenvalue nor a resonance at the threshold $\alpha^{2}=1$. The inequality (9.9) will play a crucial role in estimating a quadratic form as in the previous paragraph dealing with the absence of eigenvalues. To see this, let

$$
\mathcal{F}_{\varepsilon}:=\operatorname{span}\left\{\psi^{\varepsilon}, \phi^{\prime}, \tilde{\eta}, \phi\right\} .
$$

As before, one shows that $\operatorname{dim} \mathcal{F}_{\varepsilon}=4$, as least if $\varepsilon>0$ is sufficiently small (use that $\left\langle L_{+} \psi^{\varepsilon}, \psi^{\varepsilon}\right\rangle \rightarrow \infty$ as $\varepsilon \rightarrow 0$ ). It remains to show that for small $\varepsilon>0$

$$
\max _{f \in \mathcal{F}_{\varepsilon}} \frac{\left\langle P L_{+} P f, f\right\rangle}{\langle f, f\rangle}<1
$$

where $P$ is the projection orthogonal to $\phi$. If so, then this would imply that $A=P L_{+} P$ has at least four eigenvalues (with multiplicity) in $(-\infty, 1)$. However, we have shown above that there are exactly three such eigenvalues. To prove (9.10), 
it suffices to consider the case $f \perp \phi$. Compute

$$
\begin{aligned}
& \frac{\left\langle L_{+}\left(a \psi^{\varepsilon}+c \phi^{\prime}+d \tilde{\eta}\right), a \psi^{\varepsilon}+c \phi^{\prime}+d \tilde{\eta}\right\rangle}{\left\|a \psi^{\varepsilon}+c \phi^{\prime}+d \tilde{\eta}\right\|^{2}} \\
& =\frac{|a|^{2}\left(\left\|\psi^{\varepsilon}\right\|_{2}^{2}+M_{1}+o(1)\right)+2 \Re \lambda_{1}\left\langle a \psi^{\varepsilon}, d \tilde{\eta}\right\rangle+\lambda_{1}\|d \tilde{\eta}\|_{2}^{2}}{|a|^{2}\left\|\psi^{\varepsilon}\right\|_{2}^{2}+2 \Re\left\langle a \psi^{\varepsilon}, c \phi^{\prime}+d \tilde{\eta}\right\rangle+\left\|c \phi^{\prime}+d \tilde{\eta}\right\|_{2}^{2}} \\
& \leq \max _{x \in \mathbb{C}^{3}} \frac{\left|x_{1}\right|^{2}\left(1+\delta^{2} M_{1}+o\left(\delta^{2}\right)\right)+2 \delta \lambda_{1} \Re\left\langle x_{1} \psi^{\varepsilon}, x_{3} e_{2}\right\rangle+\lambda_{1}\left|x_{3}\right|^{2}}{\left|x_{1}\right|^{2}+2 \delta \Re\left\langle x_{1} \psi^{\varepsilon}, x_{2} e_{1}+x_{3} e_{2}\right\rangle+\left\|x_{2} e_{1}+x_{3} e_{2}\right\|_{2}^{2}}
\end{aligned}
$$

where we have set $\delta^{2}:=\left\|\psi^{\varepsilon}\right\|_{2}^{-2}$ and

$$
e_{1}=\frac{\phi^{\prime}}{\left\|\phi^{\prime}\right\|_{2}}, \quad e_{2}=\frac{\tilde{\eta}}{\|\tilde{\eta}\|_{2}} .
$$

Note that $\tilde{\eta}$ is an even function, since it is given by $\left(L_{+}-\lambda_{1}\right)^{-1} \phi$ and both $\phi$ and the kernel of $\left(L_{+}-1\right)^{-1}$ are even. Hence $e_{1} \perp e_{2}$. Set

$$
b_{j}^{\varepsilon}:=\left\langle\psi^{\varepsilon}, e_{j}\right\rangle \text { for } 1 \leq j \leq 2 .
$$

Then $b_{j}^{\varepsilon} \rightarrow b_{j}^{0}:=\left\langle\psi, e_{j}\right\rangle$ as $\varepsilon \rightarrow 0$ by the exponential decay of the $e_{j}$. Let $B^{\varepsilon}, C^{\varepsilon}$ (which depend on $\varepsilon$ ) be $3 \times 3$ Hermitian matrices so that

$$
C_{11}^{\varepsilon}:=1+\delta^{2} M_{1}+o\left(\delta^{2}\right), C_{13}^{\varepsilon}=C_{31}^{\varepsilon}:=\lambda_{1} \delta b_{2}^{\varepsilon}, C_{33}^{\varepsilon}:=\lambda_{1}
$$

and $C_{i j}^{\varepsilon}=0$ otherwise,

$$
B_{1 j}^{\varepsilon}=B_{j 1}^{\varepsilon}:=\delta b_{j-1}^{\varepsilon} \text { for } 2 \leq j \leq 3
$$

and $B_{i j}^{\varepsilon}=0$ otherwise. In view of the preceding,

$$
\max _{f \in \mathcal{F}_{\varepsilon}} \frac{\left\langle P L_{+} P f, f\right\rangle}{\langle f, f\rangle} \leq \max _{x \in \mathbb{C}^{3}} \frac{\langle C x, x\rangle}{\langle(I+B) x, x\rangle} .
$$

Clearly, the right-hand side equals the largest eigenvalue of the Hermitian matrix $\left(I+B^{\varepsilon}\right)^{-\frac{1}{2}} C^{\varepsilon}\left(I+B^{\varepsilon}\right)^{-\frac{1}{2}}=C-\frac{1}{2}(B C+C B)+\frac{3}{8}\left(B^{2} C+C B^{2}\right)+\frac{1}{4} B C B+O\left(\delta^{3}\right)$, where we have dropped the $\varepsilon$ in the notation on the right-hand side. With some patience one can check that the right-hand side equals the matrix $D$ which is given by (dropping $\varepsilon$ from the notation)

$$
\left[\begin{array}{lll}
1+\delta^{2} M_{2} & -\frac{\delta}{2} b_{1} & \frac{\delta}{2}\left(\lambda_{1}-1\right) b_{2} \\
-\frac{\delta}{2} b_{1} & \frac{\delta^{2}}{4} b_{1}^{2} & \frac{\delta^{2}}{4}\left(1-\frac{1}{2} \lambda_{1}\right) b_{1} b_{2} \\
\frac{\delta}{2}\left(\lambda_{1}-1\right) b_{2} & \frac{\delta^{2}}{4}\left(1-\frac{1}{2} \lambda_{1}\right) b_{1} b_{2} & \lambda_{1}+\frac{\delta^{2}}{4}\left(1-\lambda_{1}\right) b_{2}^{2}
\end{array}\right]+o\left(\delta^{2}\right)
$$

where $M_{2}:=M_{1}-\frac{3}{4} \lambda_{1} b_{2}^{2}+\frac{3}{4}\left(b_{1}^{2}+b_{2}^{2}\right)$. When $\delta=0$, this matrix has simple eigenvalues $1,0, \lambda_{1}<0$. When $\delta \neq 0$ but is very small, the largest eigenvalue will be close to one, of the form $1+x$ with $x$ small. We need to see that $x<0$. Collecting powers of $x$ in $\operatorname{det}(D-(1+x) I)$, we arrive at the condition

$$
\begin{aligned}
\left(1-\lambda_{1}\right) x & =\delta^{2}\left[M_{1}\left(1-\lambda_{1}\right)+b_{1}^{2}\left(1-\lambda_{1}\right)+b_{2}^{2}\left(1-\lambda_{1}\right)^{2}\right]+o\left(\delta^{2}\right) \\
& =\delta^{2}\left[M_{1}\left(1-\lambda_{1}\right)+\left(b_{1}^{0}\right)^{2}\left(1-\lambda_{1}\right)+\left(b_{2}^{0}\right)^{2}\left(1-\lambda_{1}\right)^{2}\right]+o\left(\delta^{2}\right) .
\end{aligned}
$$

We have

$$
b_{1}^{0}=\left\langle\psi, e_{1}\right\rangle=-\left\langle\left(L_{+}-1\right) \psi, e_{1}\right\rangle=-\left\langle f, e_{1}\right\rangle .
$$

On the other hand,

$$
b_{2}^{0}=\left\langle\psi, e_{2}\right\rangle=-\left\langle f, e_{2}\right\rangle+\left\langle\psi, L_{+} e_{2}\right\rangle=-\left\langle f, e_{2}\right\rangle+\lambda_{1} b_{2}^{0}
$$


and thus,

$$
b_{2}^{0}=-\left(1-\lambda_{1}\right)^{-1}\left\langle f, e_{2}\right\rangle
$$

Since $\lambda_{1}<0$ in the supercritical case, we obtain that

$$
\begin{aligned}
\left(1-\lambda_{1}\right) x & \leq\left(1-\lambda_{1}\right) \delta^{2}\left[M_{1}+\sum_{j=1}^{2}\left\langle f, e_{j}\right\rangle^{2}\right]+o\left(\delta^{2}\right) \leq\left(1-\lambda_{1}\right) \delta^{2}\left[M_{1}+\langle f, f\rangle\right]+o\left(\delta^{2}\right) \\
& =\left(1-\lambda_{1}\right) \delta^{2}\langle f+\psi, f\rangle+o\left(\delta^{2}\right)=-\left(1-\lambda_{1}\right) \delta^{2}\left\langle\left(L_{-}-1\right)^{-1} f, f\right\rangle+o\left(\delta^{2}\right)
\end{aligned}
$$

which yields that $x<0$ for $\delta$ small. But $\varepsilon>0$ small implies that $\delta$ is small and we are done.

Finally, we turn to the remaining issue of embedded eigenvalues in the essential spectrum. The argument is that of Subsection 2.1.3 of [36] generalized from $\sigma=2$ to $\sigma>2$ (in fact, it applies to any $\sigma>0$ ). Suppose that

$$
\mathcal{H} f=E f \text { with } E>1 .
$$

Then the substitution $z=\tanh (\sigma x)$ and $v(z)=f(x)$ transforms this into the following system of differential equations with meromorphic coefficients:

$$
\left(-\partial_{z}^{2}+\frac{2 z}{1-z^{2}} \partial_{z}+\frac{1}{\sigma^{2}\left(1-z^{2}\right)^{2}}\right) v-\frac{(\sigma+1)^{2}}{\sigma^{2}\left(1-z^{2}\right)} v-\frac{\sigma+1}{\sigma\left(1-z^{2}\right)} \sigma_{1} v=\frac{E}{\sigma^{2}\left(1-z^{2}\right)^{2}} \sigma_{3} v .
$$

We remind the reader that $\sigma>2$ is a scalar, whereas $\sigma_{1}=\left(\begin{array}{ll}0 & 1 \\ 1 & 0\end{array}\right), \sigma_{3}=$ $\left(\begin{array}{cc}1 & 0 \\ 0 & -1\end{array}\right)$ are Pauli matrices. The singularities are exactly $z_{ \pm}= \pm 1$ and $z_{\infty}=$ $\infty$. In fact, these are regular singular points; see Hartman [25, page 70, and in particular Fuch's Theorem 12.1 on page 85. This means that in a small punctured disk around $z=1$, say, one can find a fundamental system of solutions of the form

$$
(z-1)^{\beta}(\log (z-1))^{k} f(z) \text { with } f \text { analytic around } z=1
$$

where $\beta \in \mathbb{C}$ is a zero of the characteristic equation and $k$ is a nonnegative integer less than the multiplicity of that zero. Plugging this ansatz into the equation, one now observes that in the vicinity of $z_{ \pm}$there is a basis of solutions of the form

$$
\left(z-z_{j}\right)^{\frac{i \lambda}{2 \sigma}} e_{j 1}(z),\left(z-z_{j}\right)^{-\frac{i \lambda}{2 \sigma}} e_{j 2}(z),\left(z-z_{j}\right)^{\frac{\mu}{2 \sigma}} e_{j 3}(z),
$$

as well as

$$
\left\{\begin{array}{l}
\left(z-z_{j}\right)^{-\frac{\mu}{2 \sigma}} e_{j 4}(z) \text { if } \frac{\mu}{\sigma} \notin \mathbb{Z}, \\
\log \left(z-z_{j}\right)\left(z-z_{j}\right)^{\frac{\mu}{2 \sigma}} e_{j 3}(z)+\left(z-z_{j}\right)^{-\frac{\mu}{2 \sigma}} e_{j 4}(z) \text { if } \frac{\mu}{\sigma} \in \mathbb{Z}
\end{array}\right.
$$

where $e_{j \ell}, 1 \leq \ell \leq 4, j= \pm$, are analytic and nonvanishing functions in some disk centered at $z_{j}$, and with $E=1+\lambda^{2}$, and $\mu=\sqrt{E+1}$, as before. Since $E$ is an eigenvalue, we conclude that there would have to be a nonvanishing solution of the form

$$
\left(1-z^{2}\right)^{\frac{\mu}{2 \sigma}} \tilde{v}(z)
$$

with an entire function $\tilde{v}(z)$. However, $\tilde{v}(z)$ can grow at most polynomially since $z=0$ is a regular singular point. Hence, $\tilde{v}(z)$ is a polynomial. Setting $w=\frac{1}{z}$, this 
would imply that the equation

$$
\left(-\partial_{w}^{2}-\frac{2 w}{w^{2}-1} \partial_{w}+\frac{1}{\sigma^{2}\left(w^{2}-1\right)^{2}}\right) y-\frac{(\sigma+1)^{2}}{\sigma^{2} w^{2}\left(w^{2}-1\right)} y-\frac{\sigma+1}{\sigma w^{2}\left(w^{2}-1\right)} \sigma_{1} y
$$

$$
=\frac{E}{\sigma^{2}\left(w^{2}-1\right)^{2}} \sigma_{3} y
$$

has a solution around $w=0$ of the form

$$
y(w)=w^{-\frac{\mu}{\sigma}-n}(1+O(w))
$$

for some integer $n \geq 0$. Inserting this ansatz into (9.12) implies that $\beta=-\frac{\mu}{\sigma}-n$ would need to satisfy the quadratic equation

$$
\operatorname{det}\left\{\left(-\beta(\beta-1)+\frac{(\sigma+1)^{2}}{\sigma^{2}}\right) I+\frac{\sigma+1}{\sigma} \sigma_{1}\right\}=0 .
$$

This means that $\beta$ would have to equal one of the four choices, with $\kappa=\frac{\sigma+1}{\sigma}$,

$$
\kappa, 1+\kappa,-\kappa, 1-\kappa \text {. }
$$

Of these, only $-\kappa$ and $1-\kappa$ are possible. In other words,

$$
\frac{\mu}{\sigma}+n=\frac{\sigma+1}{\sigma} \text { or } \frac{1}{\sigma} \text {. }
$$

The latter is impossible, since $\mu>\sqrt{2}$ and $n \geq 0$. Thus, the only possibility is that

$$
0<\frac{\mu-1}{\sigma}=1-n
$$

which forces that $n=0$. However, direct calculation shows that $\left(1-z^{2}\right)^{\frac{\mu}{2 \sigma}} \vec{e}$ is not a solution of the eigenvalue equation (9.11) for any constant vector $\vec{e} \neq 0$. Indeed, if $\vec{e} \neq 0$, then in that case $\vec{e}$ would need to satisfy

$$
\begin{array}{r}
{\left[\frac{\mu^{2}}{\sigma^{2}} I+\frac{\mu}{\sigma} I-\left(\frac{\sigma+1}{\sigma}\right)^{2} I-\frac{\sigma+1}{\sigma} \sigma_{1}\right] \vec{e}=0,} \\
{\left[-\frac{\mu}{\sigma} I+\frac{E}{\sigma^{2}} \sigma_{3}+\left(\frac{\sigma+1}{\sigma}\right)^{2} I+\frac{\sigma+1}{\sigma} \sigma_{1}\right] \vec{e}=0 .}
\end{array}
$$

Thus,

$$
\left(\frac{\mu^{2}}{\sigma^{2}}+\frac{\mu}{\sigma}\right) \vec{e}=\left(\frac{\mu}{\sigma} I-\frac{E}{\sigma^{2}} \sigma_{3}\right) \vec{e}
$$

which is the same as $-\mu^{2} / E$ being an eigenvalue of $\sigma_{3}$. However, the eigenvalues of $\sigma_{3}$ are \pm 1 , and $\mu^{2}=E+1 \neq \pm E$. We are done.

We now present a simple continuity statement.

Corollary 9.3. Let $\mathcal{H}(\alpha) f^{ \pm}(\alpha)= \pm i \gamma f^{ \pm}(\alpha)$ where $\left\|f^{ \pm}(\alpha)\right\|=1$. We can choose the $f^{ \pm}(\alpha)$ to be $\mathcal{J}$-invariant, i.e., $\mathcal{J} f^{ \pm}(\alpha)=f^{ \pm}(\alpha)$. Since $\left\|f^{ \pm}(\alpha)\right\|_{2}=1$, they are therefore unique up to a sign. Choose this sign consistently, i.e., so that $f^{ \pm}(\alpha)$ varies continuously with $\alpha$. In that case there is the bound

$$
\left|\gamma\left(\alpha_{1}\right)-\gamma\left(\alpha_{2}\right)\right|+\left\|f^{ \pm}\left(\alpha_{1}\right)-f^{ \pm}\left(\alpha_{2}\right)\right\|_{2} \leq C\left(\alpha_{1}\right)\left|\alpha_{1}-\alpha_{2}\right|
$$

for all $\alpha_{1}, \alpha_{2}>0$ which are sufficiently close. Let $P_{\mathrm{im}}^{ \pm}(\alpha)$ denote the Riesz projection onto $f^{ \pm}(\alpha)$, respectively. Then one has, relative to the operator norm on $L^{2} \times L^{2}$,

$$
\left\|P_{\mathrm{im}}^{ \pm}\left(\alpha_{1}\right)-P_{\mathrm{im}}^{ \pm}\left(\alpha_{2}\right)\right\| \leq C\left(\alpha_{1}\right)\left|\alpha_{1}-\alpha_{2}\right|
$$


for all $\alpha_{1}, \alpha_{2}$ as above. Moreover, the Riesz projections admit the explicit representation

$$
P_{\mathrm{im}}^{ \pm}(\alpha)=f^{ \pm}(\alpha)\left\langle\cdot, \tilde{f}^{ \pm}(\alpha)\right\rangle,
$$

where $\mathcal{H}(\alpha)^{*} \tilde{f}^{ \pm}(\alpha)=\mp i \gamma \tilde{f}^{ \pm}(\alpha)$, and $\left\|\tilde{f}^{ \pm}(\alpha)\right\|_{2}=1$.

Proof. By Remark 9.5, $\operatorname{ker}(\mathcal{H}(\alpha) \mp i \gamma)$ is $\mathcal{J}$-invariant. Thus, $\mathcal{J} f^{ \pm}(\alpha)=\lambda f^{ \pm}(\alpha)$ for some $\lambda \in \mathbb{C}$. It is easy to see that this requires that $|\lambda|^{2}=1$. Let $e^{2 i \beta}=\lambda$. It follows that $\mathcal{J}\left(e^{i \beta} f^{ \pm}(\alpha)\right)=e^{i \beta} f^{ \pm}(\alpha)$, leading to our choice of $\mathcal{J}$-invariant eigenfunction. Using the fact that

$$
\operatorname{ker}[\mathcal{H}(\alpha) \mp i \gamma(\alpha)]=\operatorname{ker}\left[(\mathcal{H}(\alpha) \mp i \gamma(\alpha))^{2}\right]
$$

one easily obtains (by means of the Riesz projections) that

$$
\left\|(\mathcal{H}(\alpha)-z)^{-1}\right\| \lesssim|z \mp i \gamma(\alpha)|^{-1} \text { provided }|z \mp i \gamma(\alpha)|<r_{0}(\alpha) .
$$

In conjunction with the resolvent identity, this yields

$$
\left|\gamma\left(\alpha_{1}\right)-\gamma\left(\alpha_{2}\right)\right| \leq C\left(\alpha_{1}\right)\left|\alpha_{1}-\alpha_{2}\right|,
$$

as well as (9.14). However, the latter clearly implies the remaining bound in (9.13). Finally, by the Riesz representation theorem, we necessarily have that (9.15) holds with some choice of $\tilde{f}^{ \pm}(\alpha) \in L^{2} \times L^{2}$. Since $P_{\mathrm{im}}^{ \pm}(\alpha)^{2}=P_{\mathrm{im}}^{ \pm}(\alpha)$, one checks that

$$
P_{\mathrm{im}}^{ \pm}(\alpha)^{*} \tilde{f}^{ \pm}(\alpha)=\tilde{f}^{ \pm}(\alpha) .
$$

However, writing down $P_{\mathrm{im}}^{ \pm}(\alpha)$ explicitly shows that

$$
\begin{aligned}
P_{\mathrm{im}}^{+}(\alpha)^{*} & =\left(\frac{1}{2 \pi i} \oint_{\gamma}(-\mathcal{H}(\alpha)+z I)^{-1} d z\right)^{*}=-\frac{1}{2 \pi i} \oint_{\gamma}\left(-\mathcal{H}(\alpha)^{*}+\bar{z} I\right)^{-1} d \bar{z} \\
& =\frac{1}{2 \pi i} \oint_{-\bar{\gamma}}\left(-\mathcal{H}(\alpha)^{*}+z I\right)^{-1} d z
\end{aligned}
$$

which is equal to the Riesz projection corresponding to the eigenvalue $-i \gamma$ of $\mathcal{H}(\alpha)^{*}$. Here $\gamma$ is a small, positively oriented, circle around $i \gamma$. A similar calculation applies to $P_{\text {im }}^{-}(\alpha)$. Hence $\mathcal{H}(\alpha)^{*} \tilde{f}^{ \pm}(\alpha)=\mp i \gamma(\alpha) \tilde{f}^{ \pm}(\alpha)$, as claimed. In view of (9.15),

$$
\left\|\tilde{f}^{ \pm}(\alpha)\right\|_{2}^{2}=\left\|P_{\mathrm{im}}^{+}(\alpha) \tilde{f}^{ \pm}(\alpha)\right\|_{2} \leq\left\|\tilde{f}^{ \pm}(\alpha)\right\|_{2},
$$

which implies that $\left\|\tilde{f}^{ \pm}(\alpha)\right\|_{2} \leq 1$. On the other hand,

$$
1=\left\|f^{ \pm}(\alpha)\right\|_{2}=\left\|P_{\mathrm{im}}^{+}(\alpha) f^{ \pm}(\alpha)\right\|_{2} \leq\left\|f^{ \pm}(\alpha)\right\|_{2}\left\|\tilde{f}^{ \pm}(\alpha)\right\|_{2}=\left\|\tilde{f}^{ \pm}(\alpha)\right\|_{2},
$$

and we are done.

We conclude with an explicit decomposition of $L^{2} \times L^{2}$ into the stable and unstable subspaces.

Lemma 9.4. Let $\mathcal{N}=\operatorname{ker}\left(\mathcal{H}(\alpha)^{2}\right)$ and let $\mathcal{N}^{*}=\operatorname{ker}\left(\left(\mathcal{H}(\alpha)^{*}\right)^{2}\right)$ be the root spaces of $\mathcal{H}$ and $\mathcal{H}^{*}$, respectively, whereas $f^{ \pm}(\alpha)$ and $\tilde{f}^{ \pm}(\alpha)$ are as in the previous lemma. Then there is a direct sum decomposition

$$
L^{2}(\mathbb{R}) \times L^{2}(\mathbb{R})=\mathcal{N}+\operatorname{span}\left\{f^{ \pm}(\alpha)\right\}+\left(\mathcal{N}^{*}+\operatorname{span}\left\{\tilde{f}^{ \pm}(\alpha)\right\}\right)^{\perp} .
$$

This means that the individual summands are linearly independent, but not necessarily orthogonal. The decomposition (9.16) is invariant under $\mathcal{H}$. The Riesz projection $P_{s}$ is precisely the projection onto the orthogonal complement in (9.16) which is induced by the splitting (9.16). 
Proof. This is immediate from the definition of the Riesz projections. First,

$$
I-P_{s}=\frac{1}{2 \pi i} \oint_{\gamma}(z I-\mathcal{H})^{-1} d z
$$

where $\gamma$ is a simple closed curve that encloses the entire discrete spectrum of $\mathcal{H}$ and lies within the resolvent set; see (6.7). Then, on the one hand,

$$
L^{2}(\mathbb{R}) \times L^{2}(\mathbb{R})=\operatorname{ker}\left(P_{s}\right)+\operatorname{Ran}\left(P_{s}\right)=\operatorname{ker}\left(P_{s}\right)+\operatorname{ker}\left(P_{s}^{*}\right)^{\perp} .
$$

On the other hand,

$$
\operatorname{ker}\left(P_{s}\right)=\operatorname{Ran}\left(I-P_{s}\right)=\mathcal{N}+\operatorname{span}\left\{f^{ \pm}(\alpha)\right\}
$$

as well as

$$
\operatorname{ker}\left(P_{s}^{*}\right)=\mathcal{N}^{*}+\operatorname{span}\left\{\tilde{f}^{ \pm}(\alpha)\right\} .
$$

This last equality uses that $P_{s}^{*}$ is the same as the Riesz projection off the discrete spectrum of $\mathcal{H}^{*}$, as can be seen by taking adjoints of (6.7).

Remark 9.5. By inspection, all root spaces in this section are $\mathcal{J}$-invariant. This is a general fact. Indeed, one checks easily that $J \mathcal{H}(\alpha) J=-\mathcal{H}(\alpha)$. Therefore, if $\mathcal{H}(\alpha) f=i \sigma f$ with $\sigma \in \mathbb{R}$, it follows that $\mathcal{H}(\alpha) J f=-i \sigma J f$ where as usual $J=\left(\begin{array}{cc}0 & 1 \\ 1 & 0\end{array}\right)$. Hence

$$
\mathcal{J} \operatorname{ker}(\mathcal{H}-i \sigma I)=\overline{J \operatorname{ker}(\mathcal{H}-i \sigma I)}=\operatorname{ker}(\mathcal{H}-i \sigma I)
$$

for any $\sigma \in \mathbb{R}$. A similar argument shows that the root spaces at zero are also $\mathcal{J}$-invariant. In particular, one concludes from this that the Riesz projections $P_{s}, P_{\text {root }}, P_{\text {im }}$ preserve the space of $\mathcal{J}$-invariant functions in $L^{2}(\mathbb{R}) \times L^{2}(\mathbb{R})$. This can also easily be seen directly: Let $P$ be any Riesz projection corresponding to an eigenvalue of $\mathcal{H}(\alpha)$ on $i \mathbb{R}$, i.e.,

$$
P=\frac{1}{2 \pi i} \oint_{\gamma}(z I-\mathcal{H}(\alpha))^{-1} d z
$$

where $\gamma$ is a small positively oriented circle centered at that eigenvalue. Since $J \mathcal{H}(\alpha) J=-\mathcal{H}(\alpha)$, one concludes that

$$
J P J=\frac{1}{2 \pi i} \oint_{\gamma} J(z I-\mathcal{H}(\alpha))^{-1} J d z=\frac{1}{2 \pi i} \oint_{\gamma}(\mathcal{H}(\alpha)+z I)^{-1} d z .
$$

Thus, if $F=\left(\begin{array}{c}F_{1} \\ F_{2}\end{array}\right)$, then $-\bar{\gamma}=\gamma$ (in the sense of oriented curves) implies that

$$
\overline{J P F}=-\frac{1}{2 \pi i} \oint_{\gamma}(\mathcal{H}(\alpha)+\bar{z} I)^{-1} d \bar{z} \overline{J F}=\frac{1}{2 \pi i} \oint_{\gamma}(z I-\mathcal{H}(\alpha))^{-1} d z \overline{J F}=P \mathcal{J} F,
$$

so $\mathcal{J} \circ P=P \circ \mathcal{J}$, as claimed.

\section{ACKNOWLEDGEMENTS}

The authors wish to thank Fritz Gesztesy for pointing out reference [15] and for helpful comments on $\cosh ^{-2}(x)$ potentials. Moreover, they are indebted to the anonymous referee for a very careful reading and many helpful comments. 


\section{REFERENCES}

1. Artbazar, G., Yajima, K. The $L^{p}$-continuity of wave operators for one dimensional Schrödinger operators. J. Math. Sci. Univ. Tokyo 7 (2000), no. 2, 221-240. MR1768465 (2001f:34166)

2. Bates, P. W., Jones, C. K. R. T. Invariant manifolds for semilinear partial differential equations. Dynamics reported, Vol. 2, 1-38, Dynam. Report. Ser. Dynam. Systems Appl., 2, Wiley, Chichester, 1989. MR1000974 (90g:58017)

3. Berestycki, H., Cazenave, T. Instabilité des états stationnaires dans les équations de Schrödinger et de Klein-Gordon non linéaires. C. R. Acad. Sci. Paris Sér. I Math. 293 (1981), no. 9, 489-492. MR0646873(84f:35120)

4. Bourgain, J., Wang, W., Construction of blowup solutions for the nonlinear Schrödinger equation with critical nonlinearity, Annali Scuola Ecole Normale Cl.(4) 25 (1997), no.1-2, 197-215. MR.1655515 (99m:35219)

5. Buslaev, V. S., Perelman, G. S. Scattering for the nonlinear Schrödinger equation: States that are close to a soliton. (Russian) Algebra i Analiz 4 (1992), no. 6, 63-102; translation in St. Petersburg Math. J. 4 (1993), no. 6, 1111-1142. MR1199635 (94b:35256)

6. Buslaev, V. S., Perelman, G. S. On the stability of solitary waves for nonlinear Schrödinger equations. Nonlinear evolution equations, 75-98, Amer. Math. Soc. Transl. Ser. 2, 164, Amer. Math. Soc., Providence, RI, 1995. MR.1334139 (96e:35157)

7. Cazenave, T., Lions, P.-L. Orbital stability of standing waves for some nonlinear Schrödinger equations. Comm. Math. Phys. 85 (1982), 549-561. MR0677997 (84i:81015)

8. Christ, M., Kiselev, A. Maximal functions associated with filtrations, J. Funct. Anal. 179 (2001), 409-425. MR.1809116 (2001i:47054)

9. Comech, A., Pelinovsky, D. Purely nonlinear instability of standing waves with minimal energy. Comm. Pure Appl. Math. 56 (2003), no. 11, 1565-1607. MR1995870 (2005h:37176)

10. Cuccagna, S. Stabilization of solutions to nonlinear Schrödinger equations. Comm. Pure Appl. Math. 54 (2001), no. 9, 1110-1145. MR1835384 (2002g:35193)

11. Cuccagna, S., Pelinovsky, D. Bifurcations from the endpoints of the essential spectrum in the linearized nonlinear Schrödinger problem, J. Math. Phys. 46 (2005), no. 5. MR2143030 (2005m:35279)

12. Cuccagna, S., Pelinovsky, D., Vougalter, V. Spectra of positive and negative energies in the linearized NLS problem. Comm. Pure Appl. Math. 58 (2005), no. 1, 1-29. MR2094265 (2005k:35374)

13. Demanet, L., Schlag, W. Numerical verification of a gap condition for a linearized NLS equation, preprint, 2005, to appear in Nonlinearity.

14. Erdoğan, M. B., Schlag, W. Dispersive estimates in the presence of a resonances and/or an eigenvalue at zero energy in dimension three: II, preprint, 2005, to appear in Journal d'Analyse.

15. Flügge, S. Practical quantum mechanics. Reprinting of Vols. I, II in one volume. SpringerVerlag, New York-Heidelberg, 1974. MR0366248 (51:2496)

16. Fröhlich, J., Gustafson, S., Jonsson, B. L. G., Sigal, I. M., Solitary wave dynamics in an external potential. Comm. Math. Phys. 250 (2004), no. 3, 613-642. MR.2094474 (2005h:35320)

17. Fröhlich, J., Tsai, T. P., Yau, H. T. On the point-particle (Newtonian) limit of the non-linear Hartree equation. Comm. Math. Phys. 225 (2002), no. 2, 223-274. MR1889225 (2003e:81047)

18. Gang, Z., Sigal, I. M. Asymptotic Stability of Nonlinear Schrödinger Equations with Potential, preprint, 2005, to appear in Reviews in Mathematical Physics.

19. Gang, Z., Sigal, I. M. Relaxation to Trapped Solitons in Nonlinear Schrödinger Equations with Potential, preprint, 2006.

20. Gesztesy, F., Jones, C. K. R. T., Latushkin, Y., Stanislavova, M. A spectral mapping theorem and invariant manifolds for nonlinear Schrödinger equations. Indiana Univ. Math. J. 49 (2000), no. 1, 221-243. MR1777032 (2001g:37144)

21. Grillakis, M. Analysis of the linearization around a critical point of an infinite dimensional Hamiltonian system. Comm. Pure Appl. Math. 43 (1990), no. 3, 299-333. MR1040143 (91d:58231)

22. Grillakis, M., Shatah, J., Strauss, W. Stability theory of solitary waves in the presence of symmetry. I. J. Funct. Anal. 74 (1987), no. 1, 160-197. MR0901236 (88g:35169) 
23. Grillakis, M., Shatah, J., Strauss, W. Stability theory of solitary waves in the presence of symmetry. II. J. Funct. Anal. 94 (1990), 308-348. MR.1081647(92a:35135)

24. Goldberg, M., Schlag, W. Dispersive estimates for Schrödinger operators in dimensions one and three. Comm. Math. Phys. 251 (2004), no. 1, 157-178. MR2096737 (2005g:81339)

25. Hartman, P. Ordinary differential equations. Corrected reprint of the second (1982) edition. Classics in Applied Mathematics, 38. Society for Industrial and Applied Mathematics (SIAM), Philadelphia, PA, 2002. MR 1929104 (2003h:34001)

26. Hislop, P. D., Sigal, I. M. Introduction to spectral theory. With applications to Schrödinger operators. Applied Mathematical Sciences, 113. Springer-Verlag, New York, 1996. MR1361167 (98h:47003)

27. Hundertmark, D., Lee, Y. R. Exponential decay of eigenfunctions and generalized eigenfunctions of non-selfadjoint matrix Schrödinger operators related to NLS, preprint, 2005.

28. Kato, T. Wave operators and similarity for some non-selfadjoint operators. Math. Ann. 162 (1965/1966), 258-279. MR0190801 (32:8211)

29. Krieger, J., Schlag, W. Non-generic blow-up solutions for the critical focusing NLS in 1-d, preprint, 2005

30. Li, C., Wiggins, S. Invariant manifolds and fibrations for perturbed nonlinear Schrödinger equations. Applied Mathematical Sciences, 128. Springer-Verlag, New York, 1997. MR1475929 (99e:58165)

31. Merle, F., Raphael, P. Sharp upper bound on the blow-up rate for the critical nonlinear Schrödinger equation. Geom. Funct. Anal. 13 (2003), no. 3, 591-642. MR.1995801 (2005j:35207)

32. Merle, F., Raphael, P. On universality of blow-up profile for $L^{2}$ critical nonlinear Schrödinger equation. Invent. Math. 156 (2004), no. 3, 565-672. MR2061329 (2006a:35283)

33. Merle, F., Raphael, P. On a sharp lower bound on the blow-up rate for the $L^{2}$ critical nonlinear Schrödinger equation. J. Amer. Math. Soc. 19 (2006), no. 1, 37-90. MR2169042

34. Murata, M. Asymptotic expansions in time for solutions of Schrödinger-type equations. J. Funct. Anal. 49 (1) (1982), 10-56. MR0680855 (85d:35019)

35. Perelman, G. Some Results on the Scattering of Weakly Interacting Solitons for Nonlinear Schrödinger Equations. In "Spectral theory, microlocal analysis, singular manifolds", Akad. Verlag (1997), 78-137. MR1608275 (99h:35203)

36. Perelman, G. On the formation of singularities in solutions of the critical nonlinear Schrödinger equation. Ann. Henri Poincaré 2 (2001), no. 4, 605-673. MR.1852922 (2002m:35205)

37. Perelman, G. Asymptotic stability of multi-soliton solutions for nonlinear Schrödinger equations. Comm. Partial Differential Equations 29 (2004), no. 7-8, 1051-1095. MR2097576 (2005g:35277)

38. Pillet, C. A., Wayne, C. E. Invariant manifolds for a class of dispersive, Hamiltonian, partial differential equations. J. Diff. Eq. 141 (1997), no. 2, 310-326. MR.1488355 (99b:35193)

39. Raphael, P. Stability of the log-log bound for blow up solutions to the critical non linear Schrödinger equation. Math. Ann. 331 (2005), no. 3, 577-609. MR2122541 (2006b:35303)

40. Reed, M., Simon, B. Methods of modern mathematical physics. IV. Academic Press [Harcourt Brace Jovanovich, Publishers], New York-London, 1979. MR0493421 (58:12429c)

41. Rodnianski, I., Schlag, W. Time decay for solutions of Schrödinger equations with rough and time-dependent potentials. Invent. Math. 155 (2004), no. 3, 451-513. MR2038194 (2005h:35295)

42. Rodnianski, I., Schlag, W., Soffer, A. Dispersive Analysis of Charge Transfer Models, Comm. Pure Appl. Math. 58 (2005), no. 2, 149-216. MR2094850 (2005i:81181)

43. Rodnianski, I., Schlag, W., Soffer, A. Asymptotic stability of $N$-soliton states of NLS, preprint, 2003.

44. Schlag, W. Stable manifolds for an orbitally unstable NLS. Preprint, 2004, to appear in Annals of Math.

45. Schlag, W. Dispersive estimates for Schrödinger operators: A survey. Preprint, 2004, to appear in "Mathematical Aspects of Nonlinear Dispersive Equations", Princeton University Press.

46. Shatah, J. Stable standing waves of nonlinear Klein-Gordon equations. Comm. Math. Phys. 91 (1983), no. 3, 313-327. MR0723756 (84m:35111) 
47. Shatah, J., Strauss, W. Instability of nonlinear bound states. Comm. Math. Phys. 100 (1985), no. 2, 173-190. MR0804458 (87b:35159)

48. Smith, H., Sogge, C. Global Strichartz estimates for nontrapping perturbations of the Laplacian. Comm. Partial Differential Equations 25 (2000), no. 11-12, 2171-2183. MR1789924 (2001j:35180)

49. Soffer, A., Weinstein, M. Multichannel nonlinear scattering for nonintegrable equations. Comm. Math. Phys. 133 (1990), 119-146. MR1071238 (91h:35303)

50. Soffer, A., Weinstein, M. Multichannel nonlinear scattering, II. The case of anisotropic potentials and data. J. Diff. Eq. 98 (1992), 376-390. MR1170476 (93i:35137)

51. Strauss, W. A. Nonlinear wave equations. CBMS Regional Conference Series in Mathematics, 73. American Mathematical Society, Providence, RI, 1989. MR1032250 (91g:35002)

52. Sulem, C., Sulem, P.-L. The nonlinear Schrödinger equation. Self-focusing and wave collapse. Applied Mathematical Sciences, 139. Springer-Verlag, New York, 1999. MR1696311 (2000f:35139)

53. Tsai, T.-P., Yau, H.-T. Stable directions for excited states of nonlinear Schrödinger equations. Comm. Partial Differential Equations 27 (2002), no. 11-12, 2363-2402. MR1944033 (2004k:35359)

54. Weder, R. The $W_{k, p}$-continuity of the Schrödinger wave operators on the line. Comm. Math. Phys. 208 (1999), no. 2, 507-520. MR.1729096 (2001c:34178)

55. Weinstein, Michael I. Modulational stability of ground states of nonlinear Schrödinger equations. SIAM J. Math. Anal. 16 (1985), no. 3, 472-491. MR0783974 (86i:35130)

56. Weinstein, Michael I. Lyapunov stability of ground states of nonlinear dispersive evolution equations. Comm. Pure Appl. Math. 39 (1986), no. 1, 51-67. MR0820338 (87f:35023)

Department of Mathematics, Harvard University, Science Center, 1 Oxford Street, Cambridge, Massachusetts 02138

E-mail address: jkrieger@math.harvard.edu

Department of Mathematics, The University of Chicago, 5734 South University AvENue, Chicago, Illinois 60637

E-mail address: schlag@math.uchicago.edu 